\title{
LONGWAVE INFRARED SNAPSHOT IMAGING SPECTROPOLARIMETER
}

\author{
by \\ Riley Aumiller \\ Copyright $\mathbb{C}$ Riley Aumiller 2013
}

A Dissertation Submitted to the Faculty of the

COLLEGE OF OPTICAL SCIENCES

In Partial Fulfillment of the Requirements

For the Degree of

DOCTOR OF PHILOSOPHY

In the Graduate College

THE UNIVERSITY OF ARIZONA

2013 


\section{THE UNIVERSITY OF ARIZONA GRADUATE COLLEGE}

As members of the Dissertation Committee, we certify that we have read the dissertation prepared by Riley Aumiller, titled Longwave Infrared Snapshot Imaging Spectropolarimeter and recommend that it be accepted as fulfilling the dissertation requirement for the Degree of Doctor of Philosophy.

Date: $5 / 21 / 2013$

Eustace Dereniak

Date: $5 / 21 / 2013$

William Dallas

Date: $5 / 21 / 2013$

Robert Sampson

Date:

Date:

Final approval and acceptance of this dissertation is contingent upon the candidate's submission of the final copies of the dissertation to the Graduate College.

I hereby certify that I have read this dissertation prepared under my direction and recommend that it be accepted as fulfilling the dissertation requirement.

Date: $5 / 21 / 2013$

Dissertation Director: Eustace Dereniak 


\section{STATEMENT BY AUTHOR}

This dissertation has been submitted in partial fulfillment of the requirements for an advanced degree at the University of Arizona and is deposited in the University Library to be made available to borrowers under rules of the Library.

Brief quotations from this dissertation are allowable without special permission, provided that an accurate acknowledgement of the source is made. Requests for permission for extended quotation from or reproduction of this manuscript in whole or in part may be granted by the copyright holder.

Signed: Riley Aumiller 


\section{ACKNOWLEDGEMENTS}

This work wouldn't have been possible without the help and support from a great number of people. My thanks and sincere appreciation go to my advisor, Eustace Dereniak, as well as Robert McMillan and Bob Sampson for helping support and fund this project. I'm incredibly grateful to Mike Kudenov, Nathan Hagan, Heather Durko, Julia Craven, Anthony Tanbakuchi, Chris Tebow, and all of my friends and colleagues from the University of Arizona College of Optical Sciences and the Optical Detection Lab Group for their friendship, support, and engaging discussions throughout my graduate career. I would also like to especially thank Dan O'Connell from $\mathrm{H} \mathrm{Nu}$ Photonics and Michael Eismann from the Air Force Research Lab for the support and the opportunities they provided me. Additional thanks goes to Shanalyn Kemme and her coworkers from Sandia National Labs for their help in fabricating some components for the system. Thank you all for helping make this project a success. 


\section{DEDICATION}

To my parents, my wife, and my family.

Thank you for all of your love and support.

To my teachers and instructors.

Thank you for inspiring me to learn more. 


\section{TABLE OF CONTENTS}

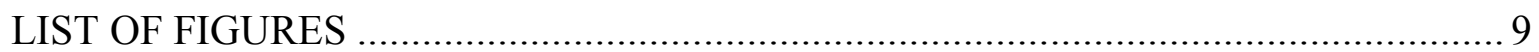

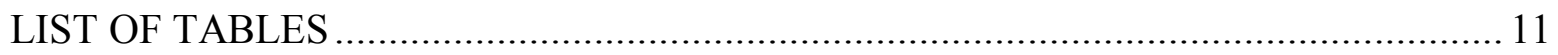

ABSTRACT …

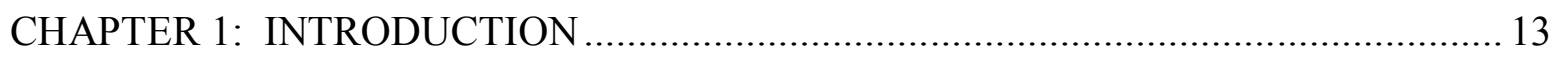

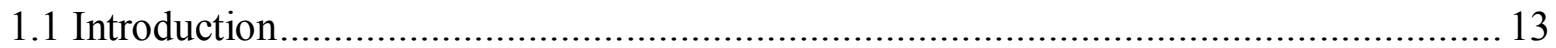

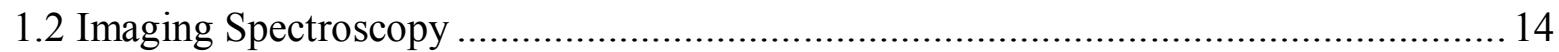

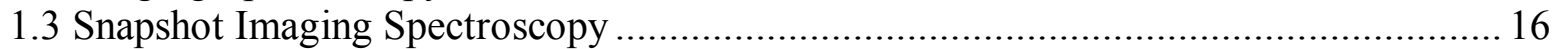

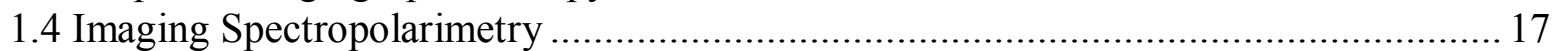

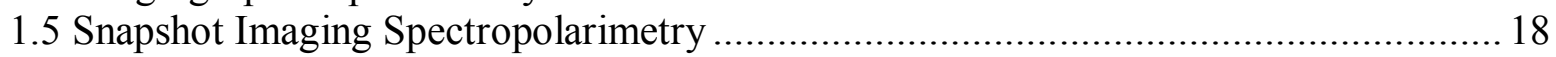

CHAPTER 2: COMPUTED TOMOGRAPHIC IMAGING SPECTROMETER (CTIS)

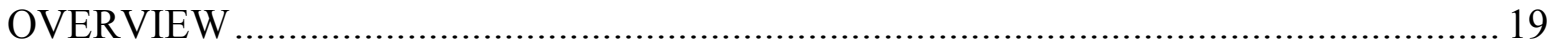

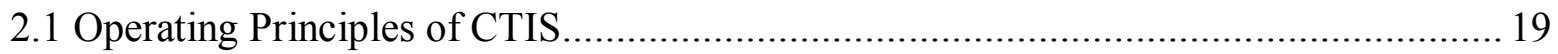

2.2 Projection of Data Cube and Reconstruction Overview............................................... 20

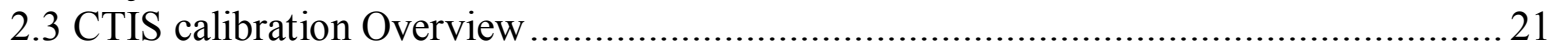

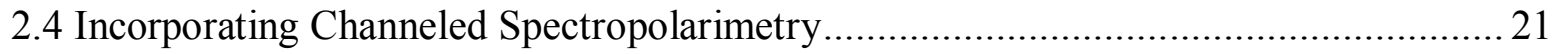

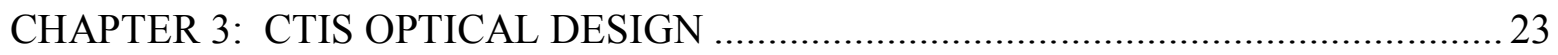

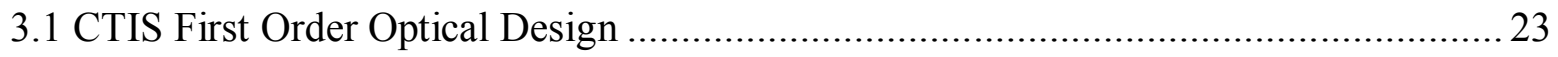

CHAPTER 4: CTIS COMPUTER GENERATED HOLOGRAM (CGH) DESIGN.......... 42

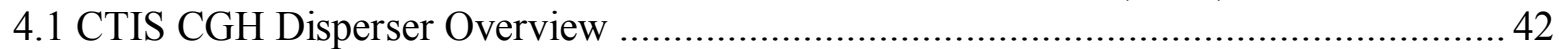

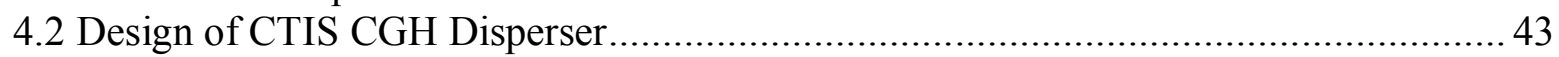

4.3 Calculating Diffraction Efficiencies for a Phase Pattern................................................ 44

4.4 How to Design the CGH Phase Pattern to Produce a Particular Pattern ......................... 50

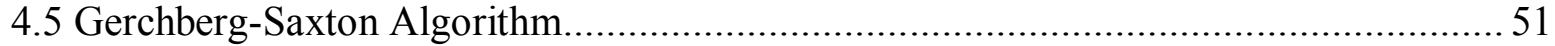

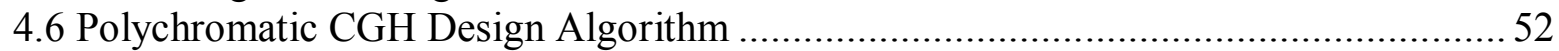

4.7 Creating a CGH For Multiple Wavelengths with Weighting Factors For Each Order ... 54

4.8 Effect of Material Dispersion in Produced CGH ........................................................... 55

4.9 Selecting Relative Diffraction Order Efficiencies to Limit Issues of SNR .................... 56

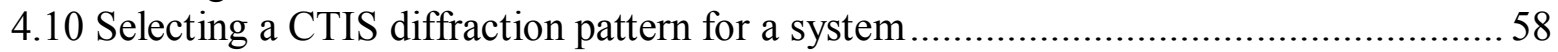

4.11 Notes and a General Rule on Comparing Diffraction Patterns .................................... 61

CHAPTER 5:CTIS CALIBRATION AND RECONSTRUCTION OVERVIEW ...............64

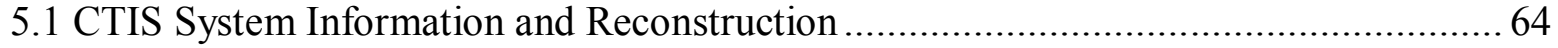

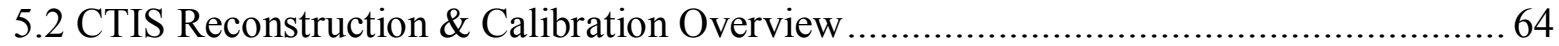

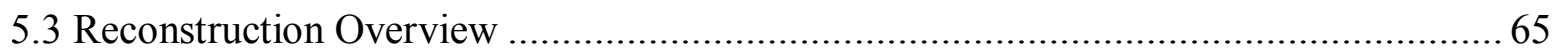

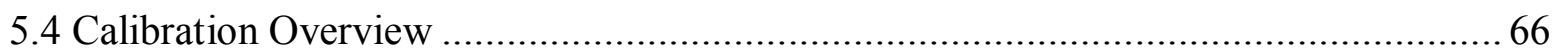

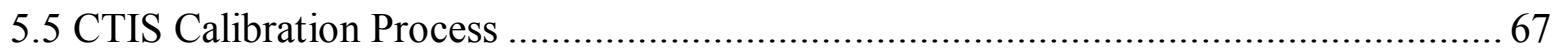

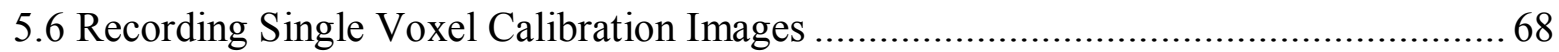




\section{TABLE OF CONTENTS - Continued}

5.7 Simplified Calibration Approach and the Shift Invariance of CTIS ........................... 69

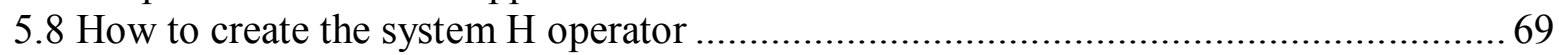

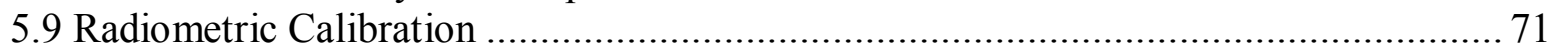

5.10 Calibration images and sampling of reconstructed data.................................. 72

5.11 Challenges in the calibration of the LWIR CTIS system ..................................... 73

CHAPTER 6: CTIS H MATRIX PROPERTIES ................................................... 76

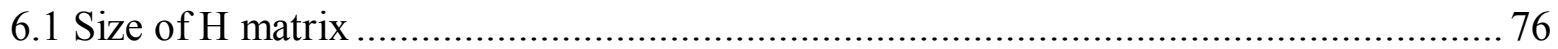

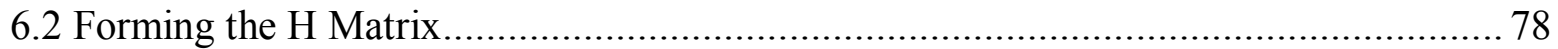

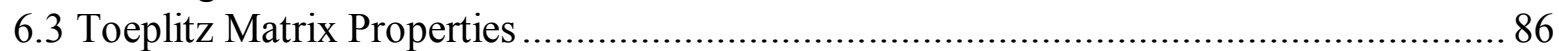

6.4 Toeplitz and Shift Invariance ....................................................................... 86

6.5 Sparse Property of the H matrix .................................................................... 87

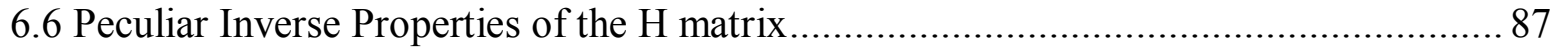

6.7 Expanding the range of invertible CTIS systems.............................................. 89

CHAPTER 7: DESCRIPTION AND COMPARISON OF CTIS RECONSTRUCTION

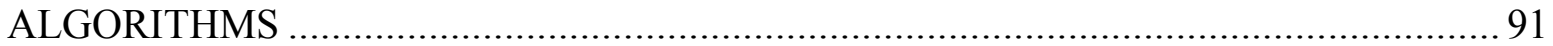

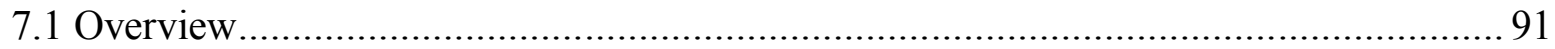

7.2 Reconstruction by Finding an Inverse of the $\mathrm{H}$ matrix ........................................ 92

7.3 Conventional Iterative Reconstruction Techniques EM and MART ........................... 93

7.4 Description of How Expectation Maximization (EM) Works ..................................... 94

7.5 Description of How MART Works .................................................................... 100

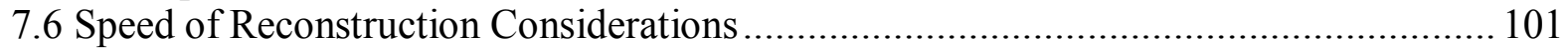

7.7 Expectation Maximization (EM) Analysis ............................................................ 103

7.8 Multiplicative Algebraic Reconstruction Technique (MART) Analysis

Implementation 1: Two Matrix vector multiplications for $\mathrm{H}^{\mathrm{T}} \mathrm{Hf}$................................. 104

7.9 Multiplicative Algebraic Reconstruction Technique (MART) Analysis

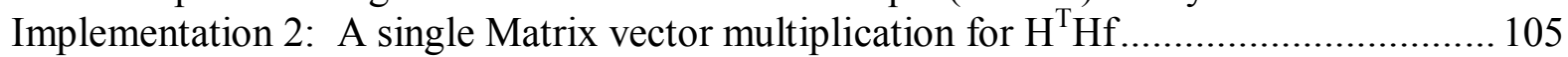

7.10 Newly Proposed Direct Inverse Reconstruction Technique: Analysis ...................... 106

7.11 Evaluating Which Reconstruction Methods are Faster ........................................... 106

7.12 Comparing Speed of Different Implementations of MART ................................... 106

7.13 Comparing Reconstruction Speed for EM and MART …..................................... 108

7.14 Comparing Reconstruction Speed for EM and Direct Left Inverse .......................... 109

7.15 How parallel processing of the CTIS H matrix multiplications could increase speeds 110

7.16 EM using Parallel Method 1 ........................................................................... 115

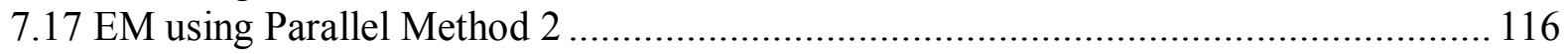

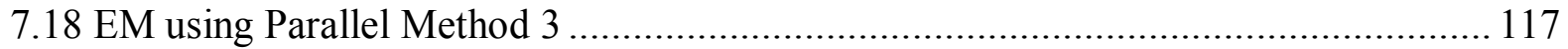

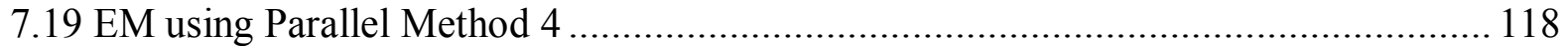

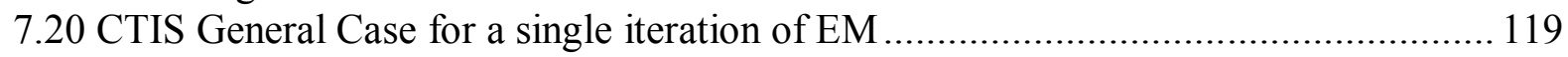

7.21 CTIS Example Case for a single iteration of EM ................................................ 120

7.22 CTIS General Case for a direct left inverse reconstruction .................................. 121

7.23 CTIS Example Case for a direct left inverse reconstruction ............................... 122

7.24 Parallel processing significantly increases reconstruction speeds ............................ 122 


\section{TABLE OF CONTENTS - Continued}

7.25 Limits on Speed and Upcoming Processing Hardware

7.26 Modifications to Increase the Speed of Reconstructions for CTIS Systems ................ 124

7.27 Direct Left Inverse Reconstructions using Modified CTIS Systems ........................... 124

7.28 Sample Operation of Modified CTIS Using Left Inverse Reconstruction ................... 127

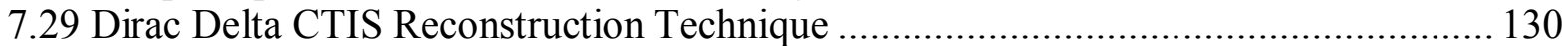

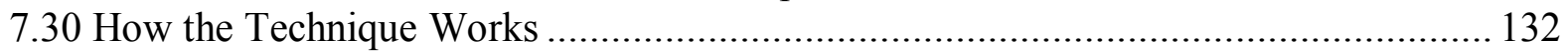

7.31 Demonstrated Reconstruction Speed Increases Using Dirac Delta Technique ............ 136

7.32 How to Implement the Dirac Delta Technique ....................................................... 139

7.33 Using Deconvolution to Mitigate Spatial Resolution Tradeoff .................................. 140

7.34 Multiple Paths to Real Time CTIS Reconstruction.................................................... 140

CHAPTER 8: DETAILS AND RESULTS FROM THE LWIR CTIS............................... 141

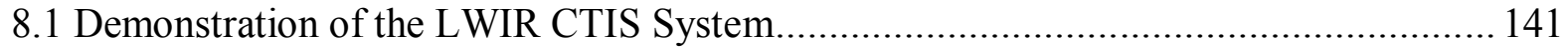

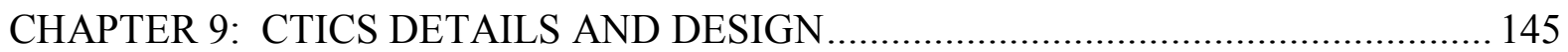

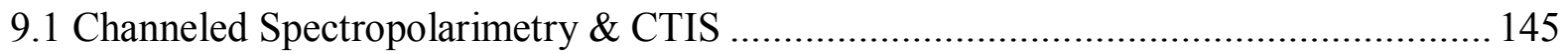

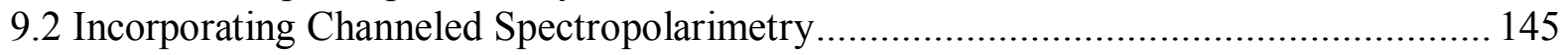

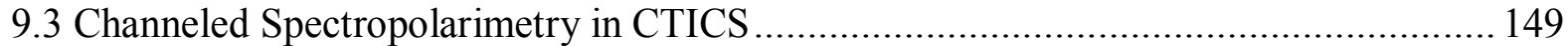

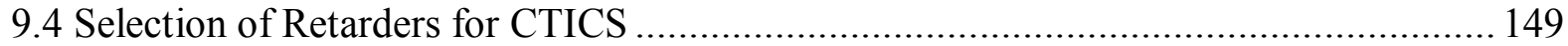

9.5 Effect of dispersion of birefringence in calculating retarder thicknesses...................... 154

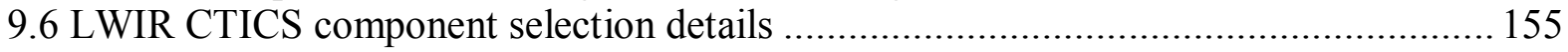

9.7 Measurements and Results from the LWIR CTICS.................................................... 157

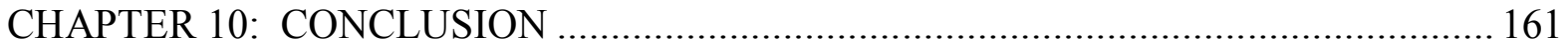

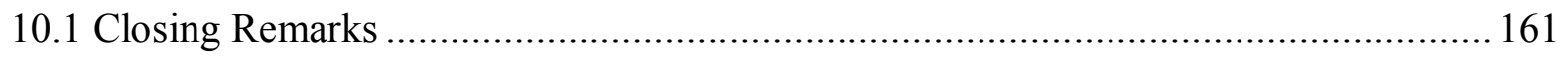

APPENDIX A: DETAILS ON TRANSFORM OF CGH TRANSMITTANCE ................. 162

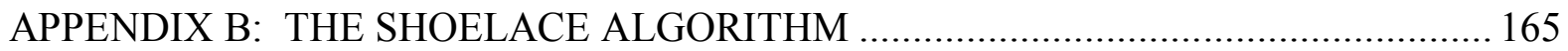

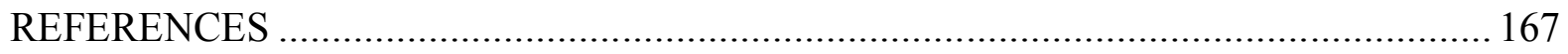




\section{LIST OF FIGURES}

Fig. 1.1. Black and white image demonstrating the spatial information.......................... 13

Fig. 1.2. Coregistered images for different bands can be used to create a color image....... 14

Fig. 1.3. Monochromatic images as horizontal plane in the $3 \mathrm{D}(\mathrm{x}, \mathrm{y}, \lambda)$ space. .................. 14

Fig. 1.4. Illustration of data acquired by various types of spectrometers ....................... 15

Fig. 1.5. Comparison of information acquired in a different spectrometer systems............ 16

Fig. 1.6. Illustration of data acquired by imaging spectropolarimeter ............................ 17

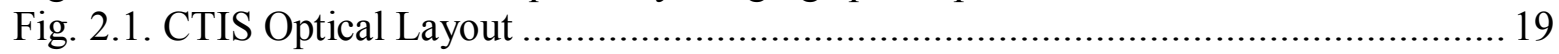

Fig. 2.2. Example image recorded by FPA in a visible CTIS system........................... 20

Fig. 2.3. Figure illustrating overlap of monochromatic images in diffraction orders.......... 20

Fig. 2.4. Illustration showing orders as projections of object cube ................................. 21

Fig. 2.5. Optical layout for CTICS .................................................................... 22

Fig. 2.6. Example of modulated spectra when viewing a polarized object....................... 22

Fig. 3.1. Effect of limited field of view on spatial and spectral multiplexing .................... 23

Fig. 3.2. Optical Layout of a CTIS ....................................................................... 25

Fig. 3.3. Illustration of how spectral components are imaged on the focal plane ............... 26

Fig. 3.4. Illustration of how spatial displacement depends upon system parameters ......... 27

Fig. 3.5. Example image of a white light source recorded by a visible CTIS system. ........ 29

Fig. 3.6. Illustration of $3 \times 3$ diffraction pattern with no overlap between the orders ........... 29

Fig. 3.7. Illustration of $5 \times 5$ diffraction pattern with no overlap between the orders ........... 30

Fig. 3.8. Illustration of outer $5 \times 5$ diffraction pattern with no overlap between the orders ... 30

Fig. 3.9. Examples showing effect of having an incorrect reimaging lens focal length ....... 33

Fig. 3.10. Selecting reimaging lens focal length to fit CTIS image on FPA...................... 34

Fig. 3.11. Illustration of how field of view of system is determined ............................. 37

Fig. 4.1. Illustration of orders produced by $1 \mathrm{D}$ grating and various $1 \mathrm{D}$ grating profiles ..... 42

Fig. 4.2. Illustration of orders produced by 2D grating and CTIS CGH profile ............... 43

Fig. 4.3. Representation of CGH unit cell showing thicknesses of each phasel. ............... 45

Fig. 4.4. Plot of the sinc function that reduces the field at each diffraction order location .. 49

Fig. 4.5. Comparison of $3 \times 3$ pattern and $7 \times 7$ pattern on the same focal plane array .......... 60

Fig. 4.6. Diagram of how reconstruction error can vary by object and CTIS pattern ......... 61

Fig. 4.7. Comparison of full $5 \times 5$ diffraction order pattern and outer $5 \times 5$ pattern ............... 61

Fig. 4.8. Illustration of the redundant diffraction orders in a $7 \mathrm{x} 7$ CTIS pattern.................. 62

Fig. 5.1. LWIR CTIS image of a soldering iron tip and exit slit of a monochromator........ 65

Fig. 5.2. Illustration of the imaging equation components in CTIS .............................. 65

Fig. 5.3. Diagram showing projections of object cube and diffraction orders .................. 66

Fig. 5.4. LWIR CTIS image of a soldering iron tip and exit slit of a monochromator......... 67

Fig. 5.5. Possible calibration setups utilizing fiber coupled monochromator output .......... 68

Fig. 5.6. Picture of a synthetic calibration image ..................................................... 75

Fig. 6.1. Representation of CTIS image with limited spatial and spectral components ....... 77

Fig. 6.2. Sample calibration image for the LWIR CTIS .......................................... 77

Fig. 6.3. Calibration images acquired with a fixed point source varying in spectrum ........ 78

Fig. 6.4. Each calibration image forms a column vector by rasterization ........................ 78

Fig. 6.5. Calibration image set used to form a matrix of column vectors ......................... 79

Fig. 6.6. CTIS H matrix is composed of multiple sets of calibration images .................... 80 


\section{LIST OF FIGURES - Continued}

Fig. 6.7. One dimension vector is used describe the object cube and data cube ................. 81

Fig. 6.8. Various calibration images for the example CTIS system ................................. 81

Fig. 6.9. Diagram of CTIS system H matrix and equivalent Block Toeplitz H matrix ........ 85

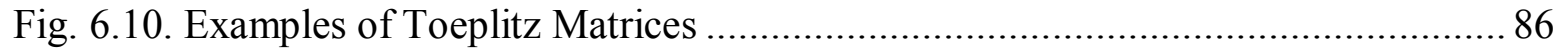

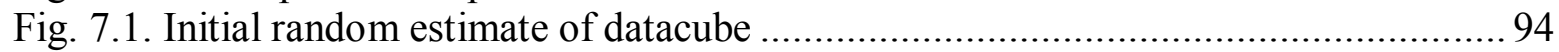

Fig. 7.2. Projection of datacube estimate to image space ….............................................. 95

Fig. 7.3. Calculating similarity between measured and estimated image ........................... 95

Fig. 7.4. Dot product of a calibration image and a CTIS image form similarity value ........ 96

Fig. 7.5. Voxel update factors are calculated using calibration images ............................... 98

Fig. 7.6. Original estimate of datacube is scaled by update factors forming new estimate .. 99

Fig. 7.7. Raw calibration image from visible CTIS system ........................................... 125

Fig. 7.8. Demonstration of Direct Left Inverse reconstruction on actual CTIS data............ 126

Fig. 7.9. Use of binning to create an effective $6 \times 6$ resolution zero order .......................... 127

Fig. 7.10. Recorded zero order image for first frame of data .......................................... 128

Fig. 7.11. Illustration of using spatial light modulator to isolate region of interest ............. 128

Fig. 7.12. Recorded zero order image for the second frame of data................................... 129

Fig. 7.13. Recorded zero order image showing maximum spatial resolution ..................... 130

Fig. 7.14. Comparison of calibration images composed of PSF's and delta functions ........ 133

Fig. 7.15. Comparison of reconstructed data using PSF H matrix and delta H matrix......... 134

Fig. 7.16. Comparing multiple iterations of PSF EM and single iteration delta $\mathrm{H}$ matrix .. 136

Fig. 7.17. Reconstructed data for a polarized source using PSF calibration data................. 138

Fig. 7.18. Reconstructed data for a polarized source using delta function calibration data.. 138

Fig. 7.19. Reconstructed data using only the outer 16 orders of image ............................ 138

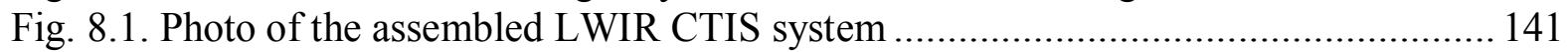

Fig. 8.2. Raw image recorded by LWIR CTIS viewing monochromator exit slit................ 142

Fig. 8.3. Raw image recorded by LWIR CTIS viewing filtered blackbody........................ 142

Fig. 8.4. CTIS image of soldering iron and multi mode output of CO2 laser ...................... 143

Fig. 8.5. CTIS image of $\mathrm{CO} 2$ laser and soldering iron (filtered and unfiltered) .................. 144

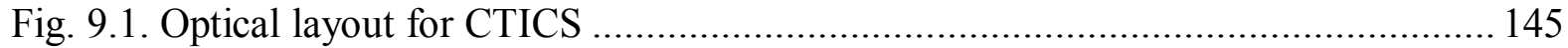

Fig. 9.2. Example of modulated spectra recorded when viewing a polarized object ........... 146

Fig. 9.3. LWIR CTIS images demonstrating change in modulation due to polarization...... 147

Fig. 9.4. Plot of seven frequency channels that make up modulated spectrum.................... 148

Fig. 9.5. Plot of seven frequency channels that make up modulated spectrum.................... 150

Fig. 9.6. Plot of index of refraction of CdS (ordinary and extraordinary axes) ................... 155

Fig. 9.7. Simulation program used to model channeled spectropolarimeter system ............. 157

Fig. 9.8. Reconstructed stokes results for unpolarized blackbody target ............................. 158

Fig. 9.9. Reconstructed stokes results for vertically polarized blackbody target ................. 159

Fig. 9.10. Reconstructed stokes results for 45 degree polarized blackbody target............... 160

Fig. 9.11. Photo of assembled LWIR CTICS system ....................................................... 160 


\section{LIST OF TABLES}

Table 1. Comparison of different CTIS diffraction order patterns and CTIS parameters .... 32

Table 2. Evaluating existence of $\mathrm{H}$ matrix left inverse for various $3 \times 3 \mathrm{CTIS}$ designs........ 88

Table 3. Evaluating existence of $\mathrm{H}$ matrix left inverse for various $5 \times 5$ CTIS designs......... 90

Table 4. $\mathrm{H}$ and $\mathrm{H}$ inverse properties of sample CTIS systems ................................... 109

Table 5. Possible reconstruction speeds using processing cores at $1000 \mathrm{MHz} \ldots \ldots \ldots \ldots \ldots \ldots . . . . . . .123$

Table 6. Sellmeier coefficients describing the index of refraction for CdS ...................... 155 


\begin{abstract}
The goal of this dissertation research is to develop and demonstrate a functioning snapshot imaging spectropolarimeter for the long wavelength infrared region of the electromagnetic spectrum (wavelengths from 8-12 microns). Such an optical system will be able to simultaneously measure both the spectral and polarimetric signatures of all the spatial locations/targets in a scene with just a single integration period of a camera. This will be accomplished by combining the use of computed tomographic imaging spectrometry (CTIS) and channeled spectropolarimetry.

The proposed system will be the first instrument of this type specifically designed to operate in the long wavelength infrared region, as well as being the first demonstration of such a system using an uncooled infrared focal plane array. In addition to the design and construction of the proof-of-concept snapshot imaging spectropolarimeter LWIR system, the dissertation research will also focus on a variety of methods on improving CTIS system performance. These enhancements will include some newly proposed methods of system design, calibration, and reconstruction aimed at improving the speed of reconstructions allowing for the first demonstration of a CTIS system capable of computing reconstructions in 'real time.'
\end{abstract}




\section{CHAPTER 1: INTRODUCTION}

\subsection{Introduction}

Due to the inherent physical properties of how optical radiation interacts with matter, light emanating from a scene will be altered or produced in a particular manner that will contain information about the original objects or scene. This light, typically from the optical spectrum consisting of wavelengths ranging from 0.4 to 12 microns, is capable of providing a variety of useful information about these objects or events. By capturing, manipulating, and measuring a portion of this light we can obtain information from the scene in order to provide object detection, characterization, classification, or identification.

This dissertation is centered around the development of a particular type of optical instrument. One way to describe this optical system, is that it is an instrument that tries to extract as much information as possible from the light it collects. Before describing the details of the instrument, it may be useful to first examine the question: What types of information does light contain?

Consider for a moment a conventional imaging system, like a standard point and shoot camera. Such camera systems are often used to record photos of people, places, or events at a particular moment in time. The recorded camera images provide two types of information: spatial information, telling us spatially where the captured light was coming from in the scene, as well as radiance information, how much of the collected light was coming from each spatial location. In a black and white camera this is accomplished by the lenses of the camera forming an image of the scene onto a sensor, mapping the incident light's various plane wavefronts onto different points on the sensor. This collected radiation is integrated over a broad spectral region and over a specified amount of time to produce a signal that is sufficiently large enough to make the recorded image useful. An example of such an image is shown in figure 1.1.

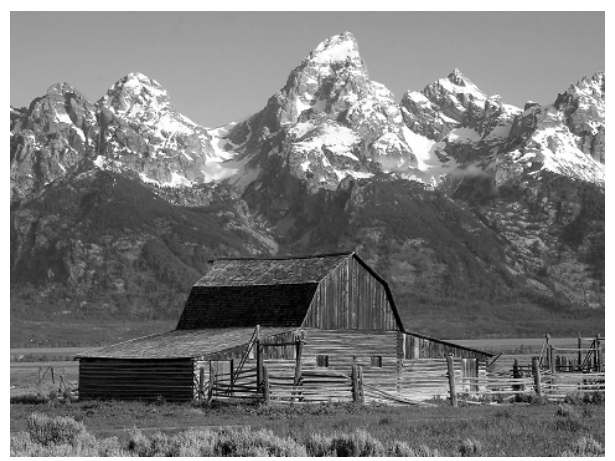

Figure 1.1: Black and white image demonstrating the spatial information and radiance information recorded in the image. $($ High radiance $=$ white Low radiance $=$ black)

In addition to containing spatial and irradiance information, the collected light will also have particular wavelength properties or spectral information. For example, most digital point and shoot cameras record color images of scenes. Compared to panchromatic black and white operation, these systems provide an additional amount of information, the approximate color of the object at a particular location, which is related to the spectral information from that location. 
This is accomplished by placing small color filters over the pixels of the sensor. These filters limit the spectral region over which light will be integrated for each pixel. Red, green, and blue spectral filters are used on the sensor to collect three spatially registered images, each corresponding to the spectral transmission of the three different color filters.

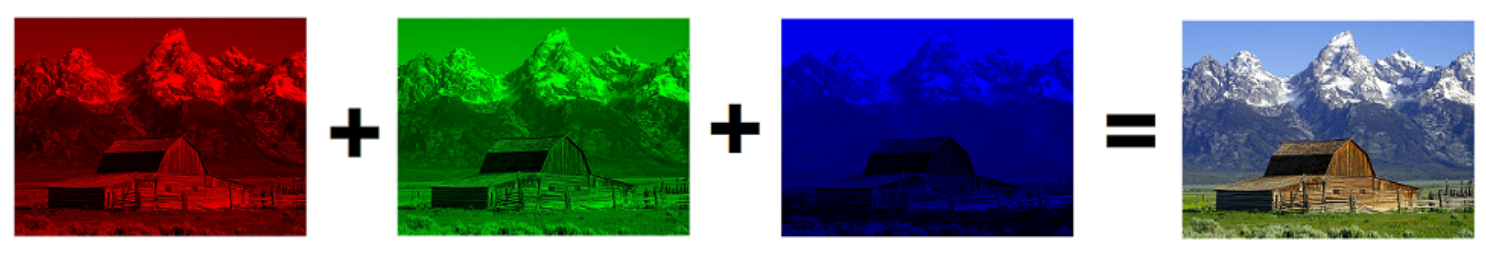

Figure 1.2: Example of how three different coregistered images corresponding to different wavelength bands can be used to create a color image.

Each of these three images contain information primarily related to a particular part of the visible spectrum. Since the spectral reflectance of visible light will be different for various objects at different wavelengths, and since each registered image is collecting light over a particular spectral band; we can see differences in the radiance values for a specific spatial location in each of the registered images. These differences in recorded image plane irradiance between the images provide information of how much light was present in each of the spectral bands, and allows for the production of a color image, as shown in figure 1.2.

This example can be taken a step farther. For the digital point and shoot camera, each of the three spectral filters corresponded to a somewhat wide spectral region. If the spectral transmission of these filters were decreased to narrower bandwidths and more filters were used to record registered images, more information about the spectral components of the incident light would be collected. Consider taking a narrowband spectral filter and placing it in front of a panchromatic camera and acquiring an image. By replacing the original spectral filter with different narrowband filters and acquiring an image for each new filter used, a large set of spatially registered, narrow spectral bandwidth images for the scene would be recorded. As more images are collected, we obtain more spatial and spectral radiance data from the scene. If the bands were narrow enough, and enough images were taken, we could estimate the spectrum of light emanating from each spatial location in the scene.

\subsection{Imaging Spectroscopy}

In imaging spectroscopy the collected spatial and spectral information is often presented as irradiance values in a three dimensional space. This three dimensional space has two spatial dimensions ( $\mathrm{x}$ and $\mathrm{y}$ ) and the third dimension being wavelength $(\lambda)$. The irradiance values will tell you how much light of a particular wavelength was recorded from a particular spatial location. So in the example of the panchromatic camera with a narrowband spectral filter in front of the system, all of the spatial information $(\mathrm{x}, \mathrm{y})$ for a particular wavelength is acquired in each image, corresponding to a horizontal plane in this 3D space, as illustrated in figure 1.3. As more and more

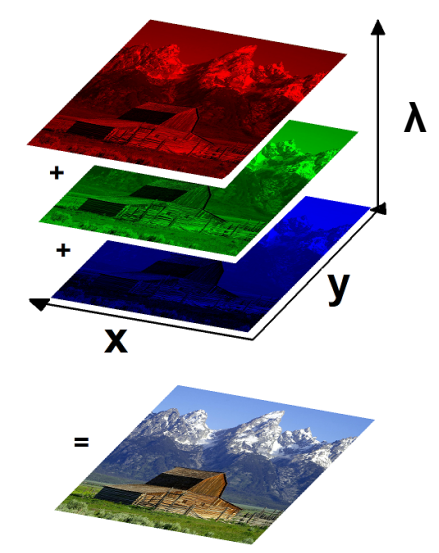

Figure 1.3: Monochromatic images as horizontal plane in the $3 \mathrm{D}(\mathrm{x}, \mathrm{y}, \lambda)$ space. 
pictures are taken, each with different filters, data spanning the entire three dimensional cube will eventually be obtained. This $3 \mathrm{D}$ cube of irradiance values is called a data cube $(\mathrm{x}, \mathrm{y}, \lambda)$.
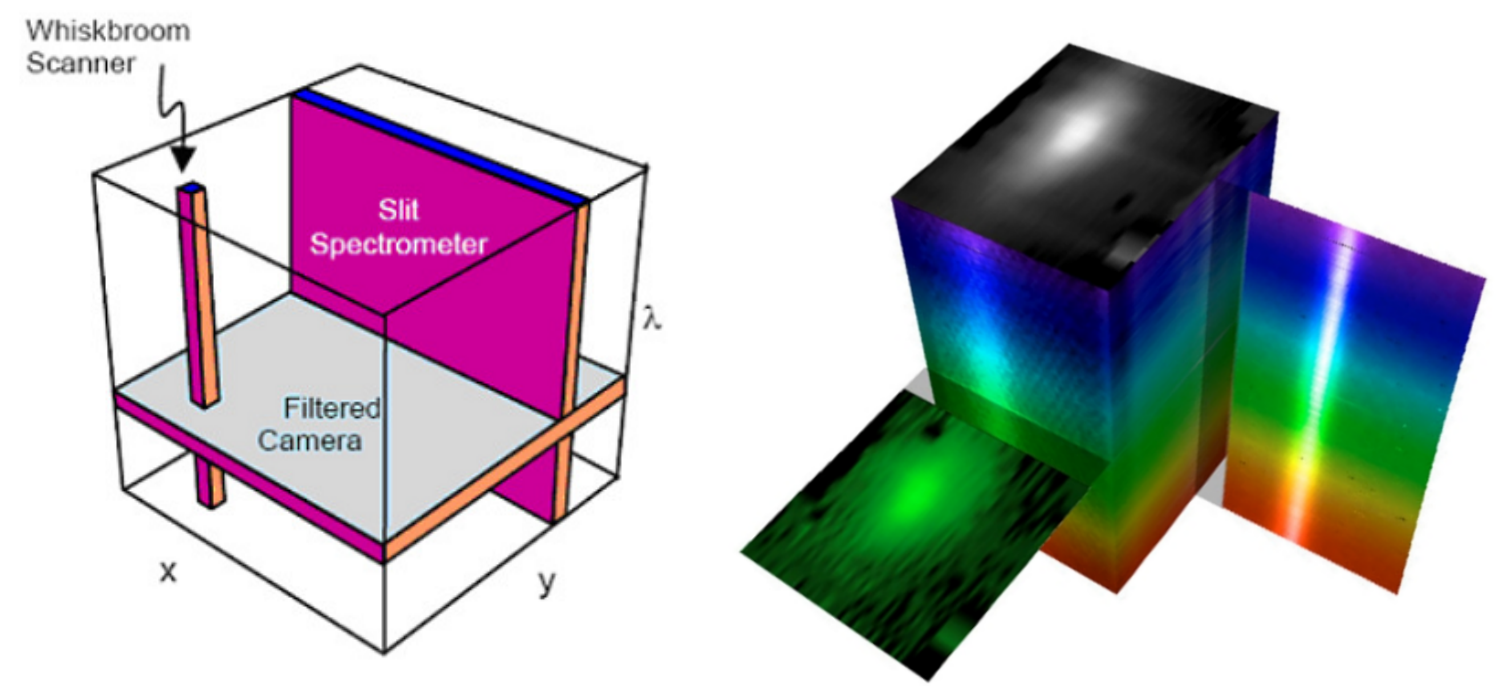

Figure 1.4: Illustration of data acquired by various types of spectrometers (left). Example of datacube collected by a visible imaging spectrometer, illustrating the monochromatic images, like those acquired from a filtered camera, as horizontal slices of the cube and spectral information from a line across in the scene, like data collected from a slit spectrometer, as a vertical slice of the datacube.

The goal of an imaging spectrometer is to fill this data cube with irradiance values. There are a variety of methods of obtaining the information needed to fill the data cube, with nearly all of the methods requiring some type of time sequential measurements or scanning mechanism to image the scene. The filtered camera approach is an example of such an imaging spectrometer. The instruments goal is to measure the inherent spatial and spectral properties of a scene, by taking a number of time sequential monochromatic images, to allow for object detection, location, characterization, classification, and/or identification based on the spatial and spectral properties of the scene.

Another example is a slit spectrometer, which images a portion of a scene onto a narrow slit. The light from this image is then separated/dispersed into its spectral components in a direction perpendicular to the slit and reimaged onto a two dimensional focal plane array. The recorded image on the focal plane array will give the spectra for points along a line in the scene, corresponding to a vertical slice in the data cube, as illustrated in figure 1.4. In order to acquire measurements to fill the data cube, the sensor must be scanned in one dimension perpendicular to the slit to cover the entire spatial extent of the scene. Another instrument, called a whiskbroom scanner, is similar to the slit spectrometer, but uses a one dimensional focal plane array to acquire the spectra from one spatial location. This instrument requires scanning in both the $\mathrm{x}$ and $y$ dimensions in order to collect data filling the data cube. 
These examples of imaging spectrometers all require some type of time sequential measurements or scanning mechanism to image the scene. The filtered camera needs a filter wheel or tunable spectral filter and must take time sequential measurements for each wavelength band. The slit spectrometer needs a one dimensional scanning mechanism to cover the entire scene, and the whiskbroom scanner will require even more complex scanning components to scan across the scene in two dimensions. These instruments have a variety of different applications; however, because of their scanning requirement these conventional systems are not well suited for observing and characterizing quick events or fast moving objects. Measurements recorded from dynamic scenes will suffer from artifacts due to the associated scanning methods.

\subsection{Snapshot Imaging Spectroscopy}

There would be a clear benefit to a system that could acquire all of the 2D spatial and spectral data simultaneously. Such a "snapshot" imaging spectrometer system would not require any scanning and would be capable of observing and characterizing quick events or fast moving objects without the artifacts of conventional systems. In the same vain that imaging systems such as your point and shoot camera, or a single frame from a video camera, are recordings of all of the spatial information from a scene at a particular moment or snapshot in time, the aim of a snapshot imaging spectrometer is to record all of the spatial and spectral information from the scene with just a single integration period of a camera. In other words, with just a single frame of data a snapshot imaging spectrometer is capable of collecting data spanning the entire three dimensional data cube as illustrated in figure 1.5.
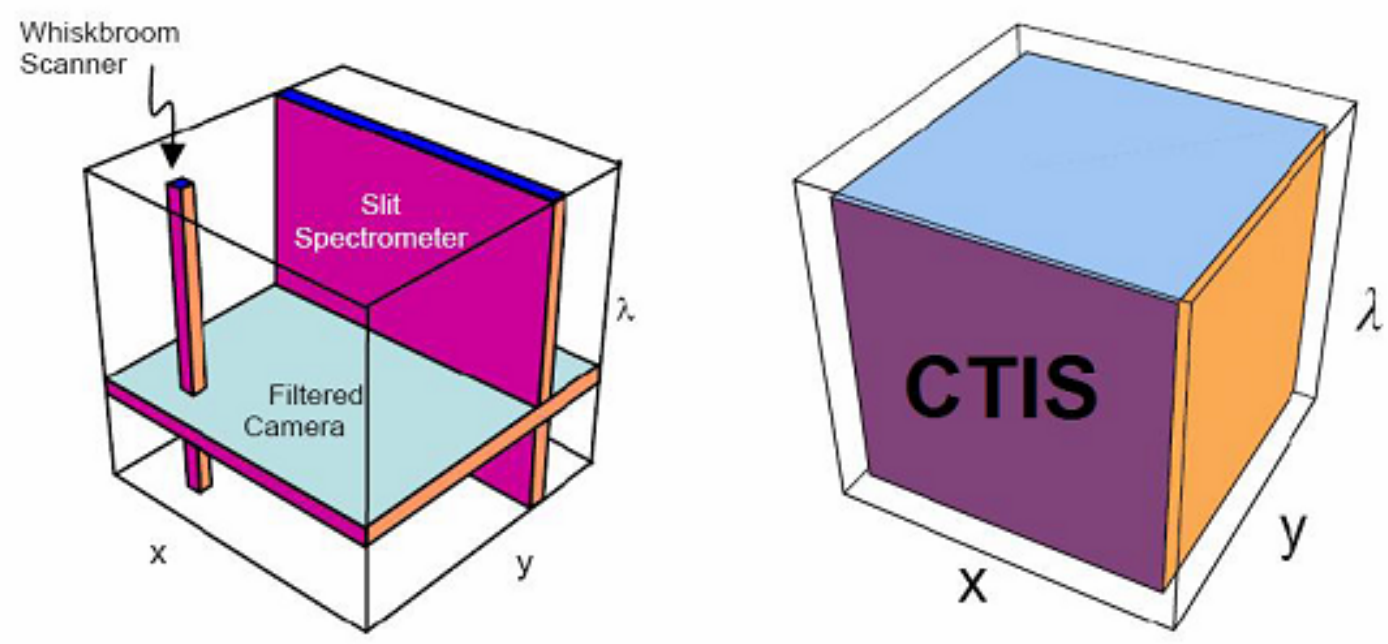

Figure 1.5: Comparison of information acquired in a single frame of data for conventional spectrometers (left) and a snapshot imaging spectrometer (right).

The instrument presented in this dissertation is based on a particular type of snapshot imaging spectrometer called a Computed Tomographic Imaging Spectrometer (CTIS). The unique capabilities and details of operation of a CTIS will be described later in the dissertation, but before moving on let's review one other type of information contained in light, polarization information. 


\subsection{Imaging Spectropolarimetry}

Polarimetry is the measurement of the state of polarization of light. It provides a description of the vector nature of the optical fields. Polarization information is often independent of the spectral characteristics of light. While spectral information can provide details on the material composition and material properties of a scene, polarization information can be analyzed to describe the shapes of surfaces or objects in a scene, their orientation, and surface properties.

Systems that are capable of measuring the spectral properties as well as the state of polarization for each wavelength band from various spatial locations of a scene are referred to as imaging spectropolarimeters.

The polarization properties of light are often mathematically expressed as a Stokes vector, consisting of the four Stokes components. Similar to how imaging spectrometers collected data within a three dimensional data cube, imaging spectropolarimeters collect data measurements within a four dimensional hypercube. The dimensions of the hypercube are 2D spatial ( $\mathrm{x}$ and $\mathrm{y}$ ), one dimension spectral $(\lambda)$, and the fourth dimension describing four Stokes polarization components. This hypercube is illustrated in figure 1.6 showing how each spatial/spectral location in the data cube now has an additional four components corresponding to the polarization properties of that spectral band of light at that particular location.
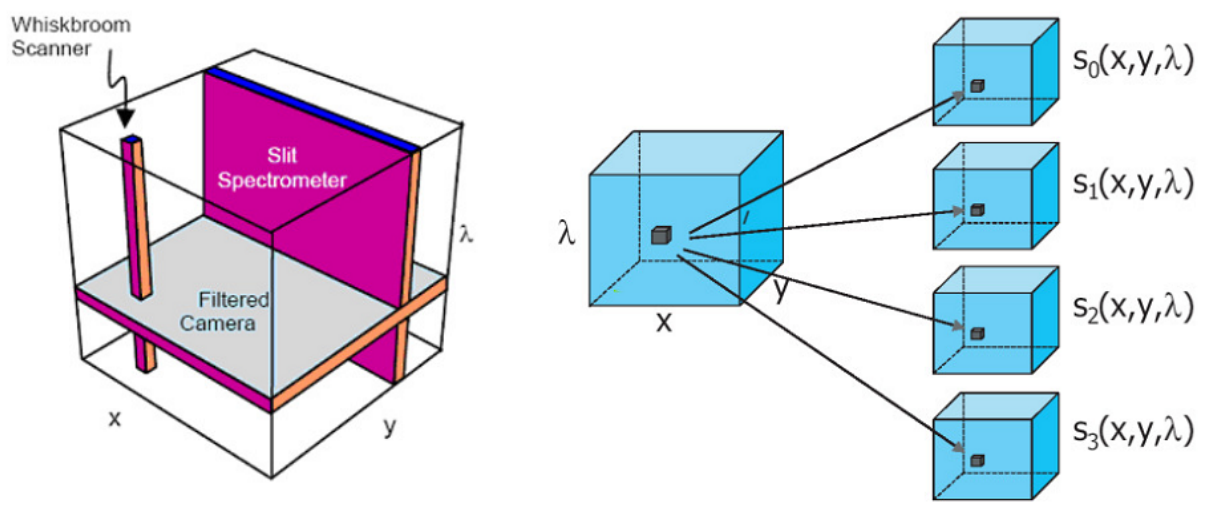

Figure 1.6: Illustration of data acquired by various types of spectrometers (left) in comparison to the complete set of data from the hypercube collected by an imaging spectropolarimeter (right).

Consider again the example of the filtered camera imaging spectrometer. In addition to having a narrowband spectral filter in front of the camera, consider placing a polarization analyzer in front of the system as well. By varying the polarization analyzer, data for the various polarization components of that particular wavelength band will be recorded across the scene. These sets of polarization measurements could be taken for each wavelength band to eventually collect data spanning the entire $4 \mathrm{D}$ hypercube. The time required to collect data is now at least four times longer since both the spectral filter and the polarization analyzer must be independently varied, and at least four different polarization measurements must be taken to describe all four stokes components. 
A variety of imaging spectropolarimeter systems have been previously implemented, collecting data in different ways, each with certain advantages and disadvantages. ${ }^{1}$ Certain instruments are especially suited to rapidly acquiring polarization data across a scene, while others are built for rapid spectral measurements. However, nearly all of these instruments still require time sequential measurements or scanning, making them not suitable for characterizing dynamic scenes.

\subsection{Snapshot Imaging Spectropolarimetry}

This dissertation is centered around the development of a snapshot imaging spectropolarimeter. It is an extremely unique imaging system instrument that can be viewed as an instrument which tries to extract as much information as possible from the light it collects. The system is capable of simultaneously recording both the spectral and polarimetric signatures of all the spatial locations/targets in a scene with just a single integration period of a camera.

Previous instruments of this type have been built in the visible ${ }^{2}$ and shortwave infrared ${ }^{3}$. This work will extend the operation into a new part of the optical spectrum and will demonstrate the first operation of a snapshot imaging spectropolarimeter in the long wavelength infrared.

In the case of visible wavelengths ( $0.4-0.7$ microns), the near infrared ( $0.7-1.1$ microns), and shortwave infrared (1.1 - 3.0 microns), reflected sunlight or reflected light from other illumination sources are the dominant source of radiation collected by passive imaging systems. In the long wavelength infrared (LWIR), wavelengths of $8-12$ microns, thermal emission is the dominant source of radiation collected. This allows LWIR systems to have both day and night operation in comparison to the previous systems since it does not rely on an external source, like the sun, for illumination. The recorded scenes for an LWIR imager will demonstrate differences in temperature and emissivity changes across the scene. In comparison to the previous systems, the LWIR snapshot imaging spectropolarimeter will be recording and characterizing signatures dominated by emission. In addition to being the first instrument of this type specifically designed to operate in the long wavelength infrared region, the presented system is also the first demonstration of such a system using an uncooled infrared focal plane array.

The system contains no moving parts and collects all of its data in a single snapshot. This snapshot capability makes it ideally suited for observing and characterizing quick events or fast moving objects without the associated scanning artifacts found in conventional imaging spectrometers and polarimeters. The rate at which the system can record the temporal nature of a target's spectral and polarimetric signature is only limited by the frame rate and sensitivity of the camera detector.

The imaging system is based on a combination of a Computed Tomographic Imaging Spectrometer (CTIS) ${ }^{4}$ and a channeled spectropolarimeter ${ }^{5}$. Only three polarization elements need to be added to a CTIS system to incorporate channeled spectropolarimetry, but to understand how the entire spectropolarimeter system works we will first review the basic design and operating principles of the Computed Tomographic Imaging Spectrometer (CTIS). 


\section{CHAPTER 2: CTIS OVERVIEW}

\subsection{Operating Principles of CTIS}

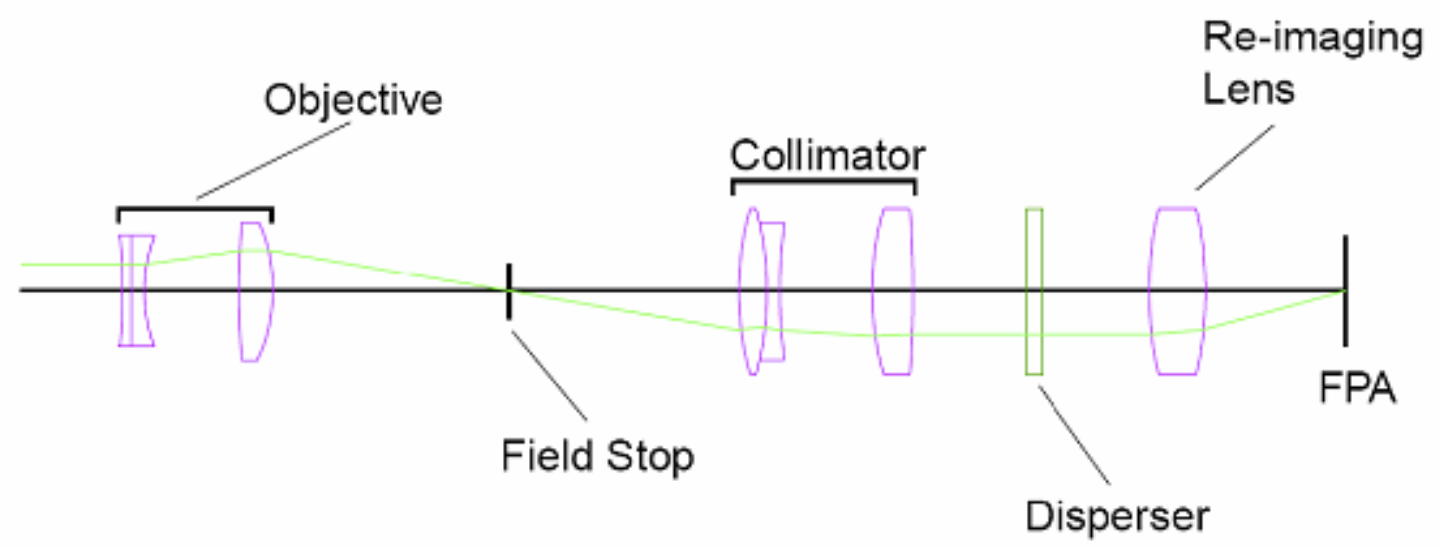

Figure 2.1: CTIS Optical Layout

The CTIS is a fairly simple optical system to analyze. The optical layout for a CTIS is shown in figure 2.1 It consists of just three lenses, a field stop, a disperser, and a 2D focal plane array. The objective lens forms an image of the scene being studied onto a field stop. The field stop is a small square aperture whose size, in combination with the focal length of the objective lens, determines the field of view of the system. The light from this intermediate image is then collimated, passed through a very specialized two-dimensional disperser or diffraction grating, and reimaged onto the focal plane array (FPA) with some minification. The amount of minification is determined by the focal lengths of the collimating and reimaging lenses.

The disperser is designed to send light into a grid of diffraction orders on the focal plane array as shown in figure 2.2. The light corresponding to the zero diffraction order of the disperser forms a panchromatic image of the scene at the center of the focal plane array. In the other diffraction orders, the image of each wavelength component of the scene is formed at a different spatial location in each diffraction order. These overlapping monochromatic images in each order cause a multiplexing of the spatial and spectral data, producing a varying overlap or "spectral smear" of the data in each of the diffraction orders, as shown in figure 2.3. The overlap of the spatial and spectral data/multiplexing will be different in different diffraction orders depending upon the orientation of that order from the center of the image and the amount of dispersion in the diffraction order. In other words, the "spectral smear" will have different lengths and will be in different directions for each of the diffraction orders in the image. 

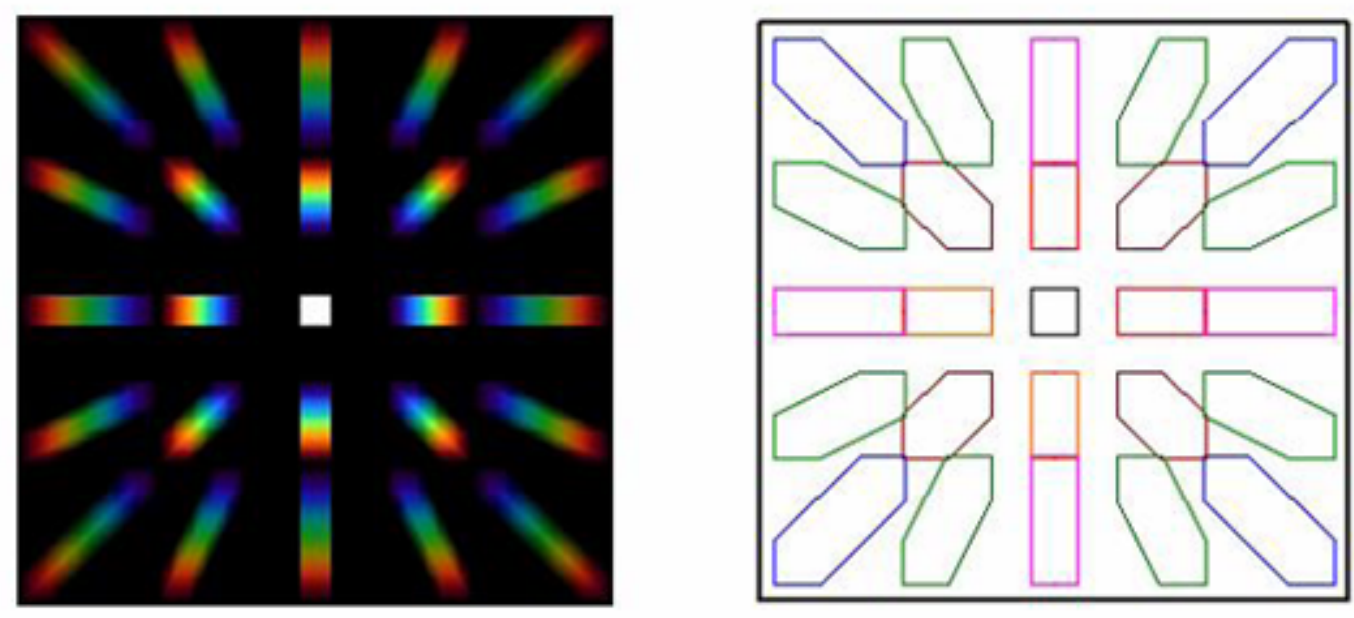

Figure 2.2: Example image recorded by FPA in a visible CTIS system (left). Diagram illustrating the location of the diffracted orders from the disperser on FPA (right).

The size of the zero order on the focal plane array determines the full spatial resolution of the scene image. The CTIS aims to measure the spectra at each pixel location in the scene over the specified spectral region of interest. The amount of dispersion or spectral spread per pixel in the outer diffraction orders will determine maximum spectral resolution of the CTIS system.

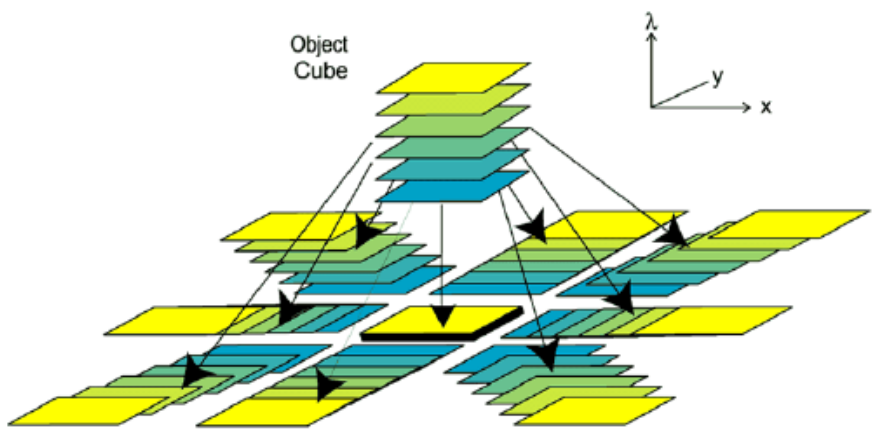

Figure 2.3: Figure illustrating the different monochromatic images of the object cube, like stacked playing cards in a deck of cards. The overlapping monochromatic images in each diffraction order can be thought of as spreading the deck of cards out in a particular direction for each order. Since the monochromatic images often overlap each other, the recorded pixel data will be a combination of spatial and spectral information from multiple points in the cube.

\subsection{Projection of Data Cube and Reconstruction Overview}

One can interpret the diffraction orders recorded on the focal plane as a collection of projections, or shadows, of a $3 \mathrm{D}$ object cube onto the $2 \mathrm{D}$ focal plane. This is illustrated in figure 2.4. By considering each of the diffraction orders as projections of the $3 \mathrm{D}$ object cube onto the $2 \mathrm{D}$ focal plane we can use the same mathematical procedures of tomographic reconstruction that are used in medical computed tomography (CT systems) to reconstruct an estimate of the original higher 
dimension 3D object from the multiple lower dimension 2D projection images. It is this reconstruction process, in combination with the various diffraction orders from the disperser, that allows the CTIS to determine the spectra at each point in the scene with a single snapshot.

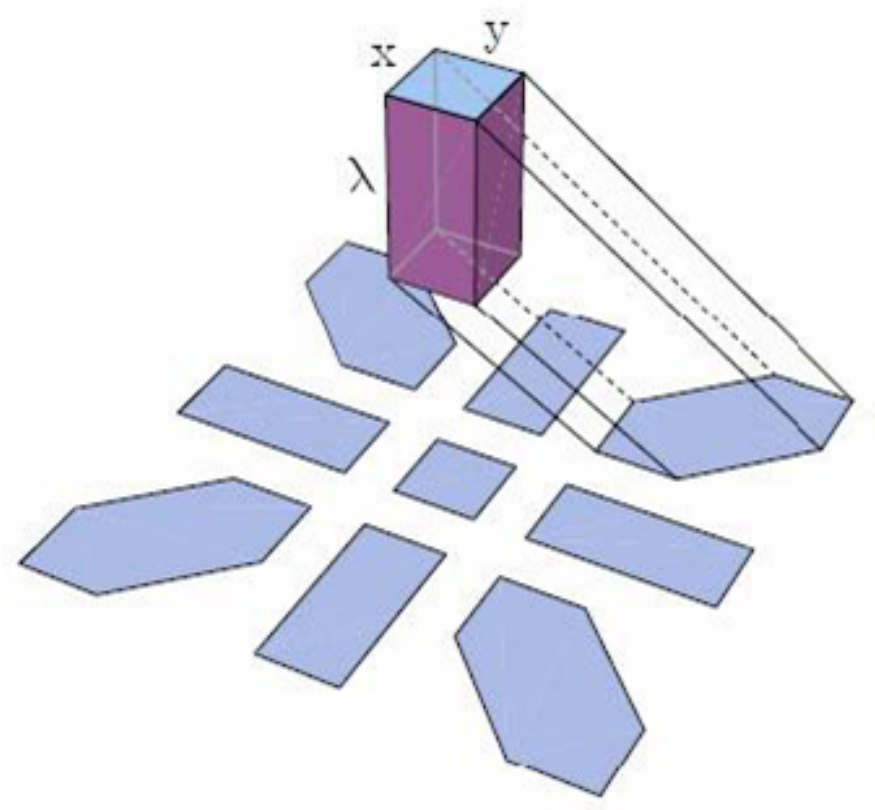

Figure 2.4: Illustration showing a diffraction order of the CTIS system as a projection or shadow of a three-dimensional object cube $(x, y, \lambda)$.

\subsection{CTIS calibration Overview}

This reconstruction process will be dependent upon some existing knowledge of the optical system. A set of calibration images must first be acquired by the CTIS system to measure how each of the defined spatial and spectral components of the object datacube map to particular pixel locations and pixel values in the recorded focal plane image. In the reconstruction of the datacube, the multiplexed spatial and spectral information in the recorded raw image will be analyzed taking into account this mapping information from the calibration data. The produced result will be a reconstructed estimate of the spectra from each spatial location in the image. This reconstructed data is given as different weighting values for all of the possible spatial and spectral components of the datacube defined in the calibration data. The capabilities of the CTIS system can be extended to include polarization state measurements by incorporating channeled spectropolarimetry, allowing the system to function as a snapshot imaging spectropolarimeter.

\subsection{Incorporating Channeled Spectropolarimetry}

To incorporate channeled spectropolarimetry ${ }^{6}$ into a CTIS system only three polarization elements need to be added ${ }^{7}$, which can be seen in figure 2.5. The added elements are placed in the collimated space of the CTIS, before the CGH, and consist of two thick birefringent crystal retarders, with their fast axes oriented at 0 and 45 degrees respectively, and an analyzing polarizer oriented at 0 degrees. These added components will encode the polarization information of the light into intensity modulations across the spectra as shown in figure 2.6. By 
carefully analyzing these recorded intensity modulations we will be able to reconstruct all four Stokes components for all the wavelength bands of each spectrum and at all the spatial locations in the scene, all with just a single frame of data. We refer to this combined system as a Computed Tomographic Imaging Channeled Spectropolarimeter (CTICS).

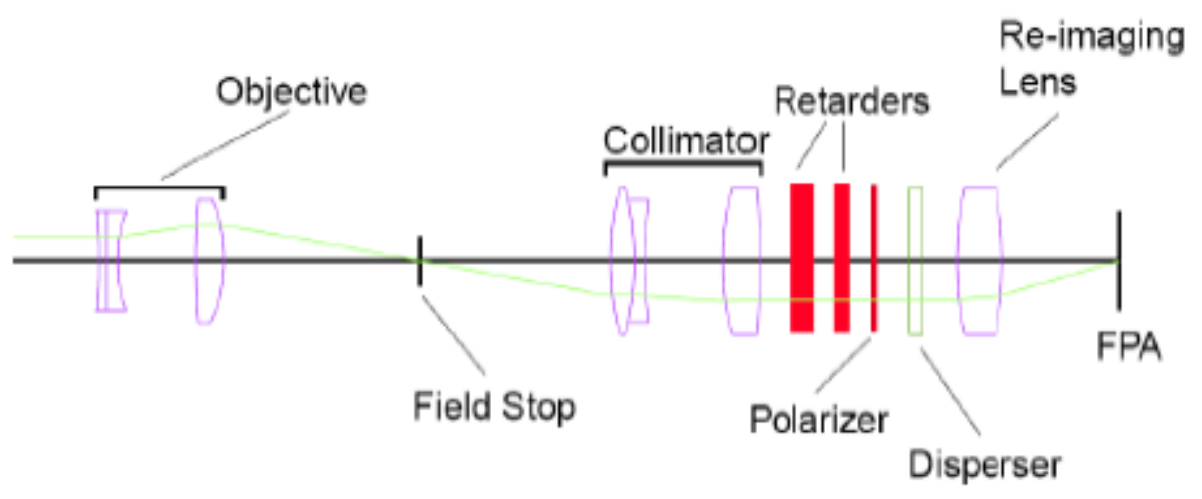

Figure 2.5: Optical layout for CTICS

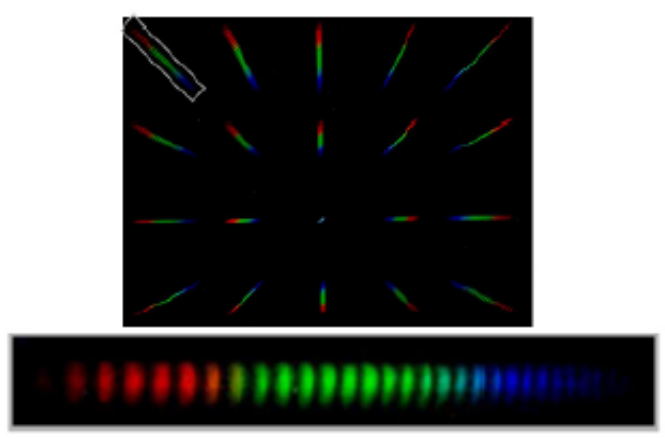

Figure 2.6: Example of modulated spectra recorded when viewing a polarized broadband object with a visible CTICS system.

We can see how the spectral dependence of the Stokes components are encoded into intensity modulations in the spectra by carefully analyzing the added components of the system. Some more details on channeled spectropolarimetry and the reconstruction of the Stokes polarization component spectra from the recorded modulations are presented in chapter 9 . However, the following chapters will cover the specific details of the CTIS system including a step-by-step process for the design of a CTIS system, the first order selection of optical components, and the details of designing the specialized disperser element. 


\section{CHAPTER 3: CTIS OPTICAL DESIGN}

\subsection{CTIS First Order Optical Design}

The goal of the optical design for an imaging spectrometer is to select the parameters of the optical components such that the system produces an image that will allow for the determination of the wavelength properties of light captured from each spatial location in the scene.

Previous work has illustrated how the CTIS system is related to a slit spectrometer ${ }^{8}$ but differs by having multiple diffraction orders in two dimensions and having the slit (or a square field stop in the $2 \mathrm{D}$ case) opened very wide. The problem the spectrometer system must deal with, since the slit or field stop is opened very wide, is the overlap/multiplexing of the spatial and spectral data from the scene on a given pixel in the focal plane. By narrowing the field of view of the system, controlling which diffraction orders will be used for measurements, adjusting the amount of dispersion in each of the diffraction orders, and by adjusting the size of the produced image relative to size of the focal plane array, the amount of multiplexing in the system can be reduced and enough unique information can be obtained such that the recorded image will allow us to produce useful spatial and spectral reconstructed data for the scene. Specifically for the design of a CTIS imaging spectrometer system, the goal is to produce an image similar to what is presented in the right image of figure 3.1 as opposed to a system with a much wider field of view and too much overlap as shown in the left image of figure 3.1.
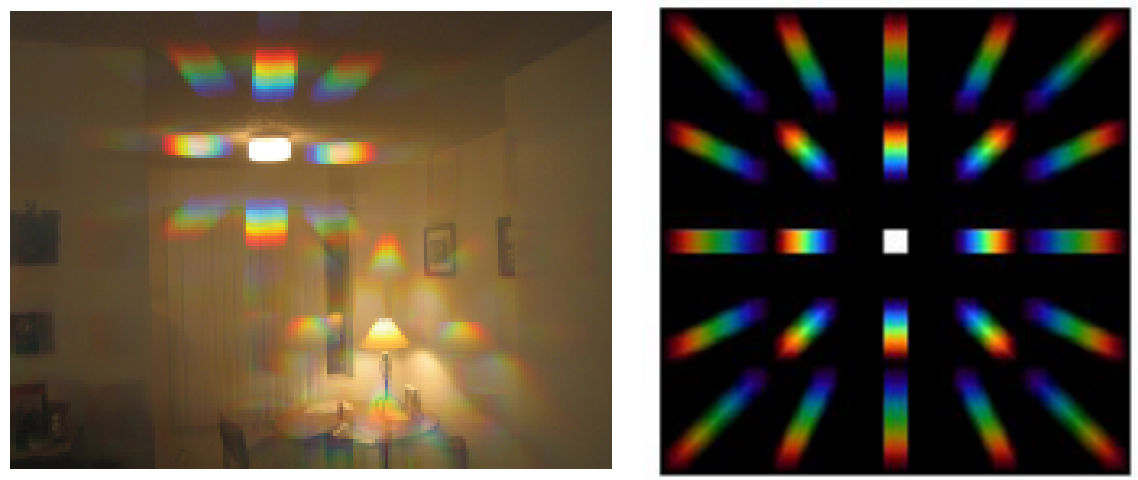

Figure 3.1: (left) An example image obtained by placing a pair of crossed gratings in front of a camera. This system has a very wide field of view with an excessive amount of multiplexing. Note that the diffraction orders/spectral components of the objects overlap with the spatial components of other objects. (right) An example image from a CTIS system. By decreasing the field stop size, the field of view of the CTIS system is far more limited and the amount of multiplexing recorded in the diffraction order is significantly reduced. 
In order to complete a first order optical design for a CTIS system, the following 5 steps need to be followed:

1) Select the CTIS diffraction order pattern

2) Specify the system's wavelength range

3) Specify initial values for the physical size of the field stop, h; the grating period, d; and the collimating lens focal length, $\mathrm{f}_{\mathrm{col}}$, so there is no overlap of the diffraction orders in the pattern. Use the table given on page 32 to determine the initial relative values of these parameters for various diffraction patterns.

4) Specify the reimaging lens focal length, $f_{\text {reim }}$, to scale the produced pattern to fit on the specified focal plane. Use equation (3.11) given on page 34 to calculate appropriate reimaging lens focal lengths.

5) Some of the initial parameter values will likely need to be adjusted, as well as selecting the objective lens focal length, to meet certain manufacturing and performance requirements for the grating, system field of view requirements, dealing with a limited availability of lens focal lengths, etc. A set of rules are presented that will scale the selected parameter values, but will not change the design, size, and location of the recorded CTIS image, ensuring that the CTIS image will still fit on the focal plane array. Follow the specified rules beginning on page 35 to scale the initial values to meet your requirements. Once finished, the specifications for your first order optical design for a CTIS system will be complete.

Details on each of these steps are presented below.

In the first step, the diffraction order pattern for the CTIS is selected. There are a variety of different diffraction order patterns that can be used in the system. Some of the basic patterns are presented in Table 1 on page 32. This selection is an especially important part of the design of the CTIS system because the diffraction pattern, in combination with the resolution of the focal plane, will be the primary factors that determine the spatial and spectral resolution of the system. Certain patterns will have a larger zero order, offering more spatial samples of the scene, while other patterns will have more dispersion in the orders producing more spectral samples for each spatial location. There are many tradeoffs in this diffraction pattern selection, which will be described in the following chapter, but the selection of the diffraction pattern must be made to try and meet the resolution requirements for the imaging spectrometer.

The second step, specifying the wavelength range for the system, should be fairly obvious to the designer. The wavelength range of the measured spectral data will determine the type of focal plane detector used, the lens materials used, and the types of spectral feature information that will be measured with the system. In some cases it is useful to specify this wavelength range based on the sensitivity range of the detector. Otherwise a spectral bandpass filter with a transmission window set to the specified wavelength range may need to be used in order to avoid overlap between the lower and higher diffraction orders in the recorded image.

Step three involves selecting parameter values to eliminate overlap of the various diffraction orders for the specified wavelength range, and thus decrease the multiplexing in the system. For example in the right image of figure 3.1 the diffraction orders are well separated and the red wavelengths of the lower orders do not overlap or multiplex with the blue wavelength images of 
the next higher diffraction orders. There are many optical design parameters that will control this overlap, and the selection of these parameters will depend upon the diffraction pattern used in the system. In order to avoid overlap between the orders, you must understand what controls the size and locations of the diffraction orders in the recorded image. To investigate the size and location of the diffraction orders, let's review the optical layout of the system, examine what parameters determine the size of the zero diffraction order, calculate the diffraction order locations of each wavelength for a broadband point source on the optic axis, and relate that information to the location and size of the monochromatic images composing the diffraction orders when viewing a full field broadband scene. With this information, we can then see what relative parameter values are needed to avoid overlap for a variety of different CTIS diffraction patterns.

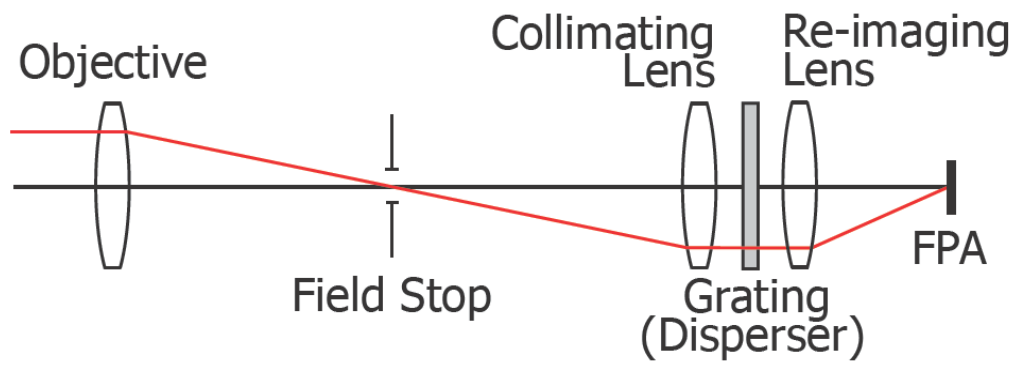

Figure 3.2: Optical Layout of a CTIS

To determine the location and size of the diffraction orders, recall the optical layout for the CTIS, shown in figure 3.2, and consider a CTIS system without a grating or disperser. The objective lens will form an image of the scene onto the square aperture of the field stop. The field stop blocks the light from this intermediate image except for light passing through the small square aperture. The combination of the collimating lens and re-imaging lens will then relay the apertured intermediate image from the field stop onto the focal plane array with some minification or magnification given by the ratio of the focal length of the reimaging lens to the focal length of the collimating lens. Since the aperture at the field stop blocks all of the light from the intermediate image except for the small square open area, the resulting size of the relayed image on the focal plane is given by

$$
\text { Size of Scene Image on Focal Plane }=\mathrm{h}\left(\frac{f_{\text {reim }}}{f_{\text {col }}}\right)
$$

where $h$ is the physical length of the square opening of the field stop aperture. Provided that all of the elements are aligned correctly, this minified or magnified image of the scene will be located at the center of the focal plane array.

Now consider placing the grating back into the CTIS system. Some of the light passing through the grating will not be diffracted at all. This light travelling directly through the grating is referred to as the zero order of the grating, and will form an image on the focal plane in the same way as the case described above. This zero order will be a minified/magnified panchromatic relayed image of the field stop with some attenuation given by the diffraction efficiency of the 
zero order. So the location of the zero order will be at the center of the focal plane with its size given by:

$$
\text { Zero Order Image Size }=\mathrm{h}\left(\frac{f_{\text {reim }}}{f_{\text {col }}}\right)
$$

Let's now look at how the other diffraction orders are mapped onto the focal plane. From the basic grating equation we know that when light is normally incident onto a grating, the exiting light will be separated out into its spectral components, illustrated in the left image of figure 3.3, with each wavelength component propagating at a different angle in each diffraction order. Consider a scene that consists of a single broadband point centered on the optical axis of the system. From the layout of the CTIS we know that this object will be imaged onto the optical axis at the field stop and the collimating lens will produce a plane wave normally incident on the grating. In this case, the grating will separate the light into various monochromatic plane waves travelling in different directions for each of the diffraction orders. These plane waves hit the reimaging lens, and depending upon the propagation angle of the various plane waves, the lens will focus the light to a particular spatial location on the focal plane, as illustrated in the right image of figure 3.3 .
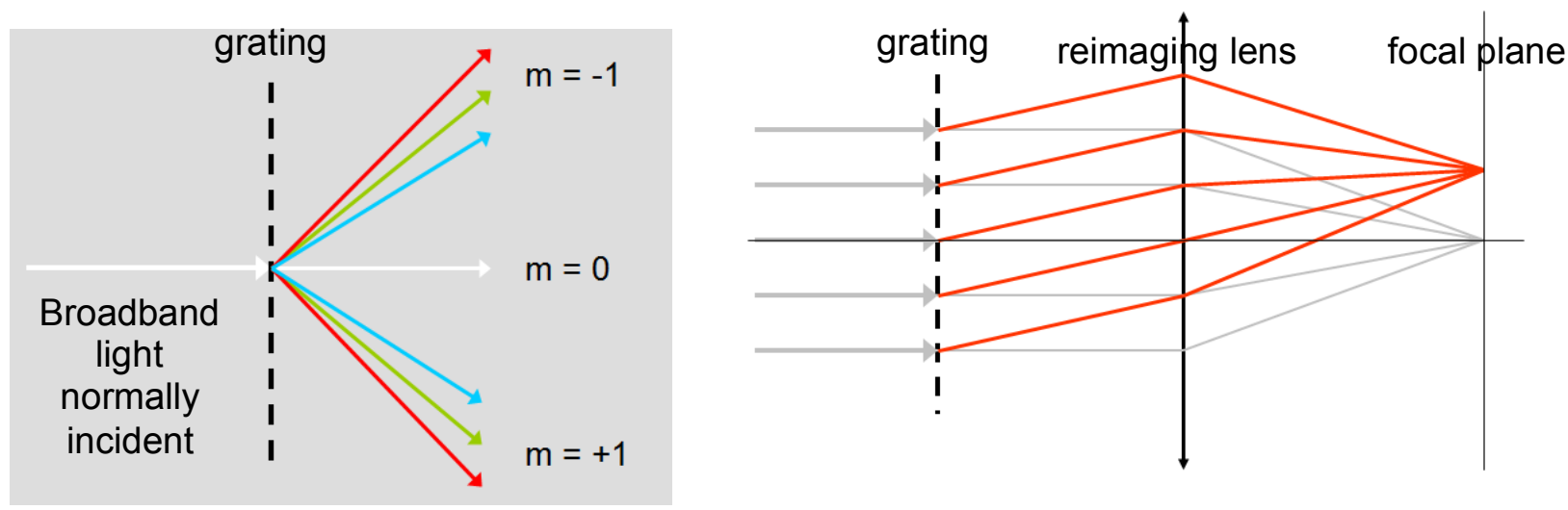

Figure 3.3: (left) Illustration of how spectrally broadband light normally incident on a grating is broken into its various spectral components for the outer diffraction orders. (right) Illustration of how the combination of grating and reimaging lens will image the various spectral components of the normally incident broadband light to different locations on the focal plane.

The location of where these various wavelength components of the diffraction orders are imaged will each have a particular displacement from the zero order image/center of the focal plane. We can determine the location of the various diffraction order wavelength components by calculating the following: 
Starting with the normally incident grating equation

$m \lambda=d \sin \left(\theta_{\text {diff }}\right)$

Solve for the angle of diffraction

$$
\theta_{d i f f}=\sin ^{-1}\left(\frac{m \lambda}{d}\right)
$$

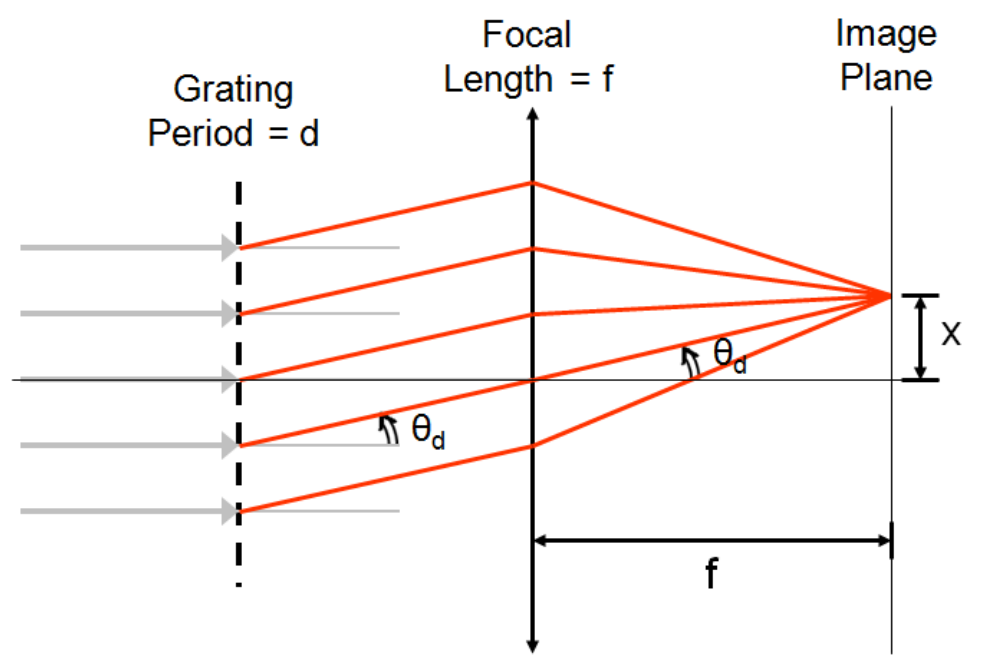

Figure 3.4: Illustration of how the displacement for the various spectral components of the normally incident broadband light, $\mathrm{x}$, is related to the diffraction angle from the grating, $\theta_{\mathrm{d}}$, and the focal length of the reimaging lens, $f$.

To first order, all of the rays incident on the thin lens will be focused to a single spot on the image plane as illustrated in figure 3.4. Noting that the angle of propagation of the diffracted ray which travels through the center of the thin lens will not be changed by the lens, we can easily determine the relationship between the diffracted angle, the focal length of the thin lens, and the physical displacement of the imaged point from the optic axis, $\mathrm{x}$, on the image plane.

$$
\tan \left(\theta_{\text {diff }}\right)=\frac{x}{f}
$$

Solve for the physical displacement $\mathrm{x}$

$$
x=f \times \tan \left[\sin ^{-1}\left(\frac{m \lambda}{d}\right)\right]
$$


Applying the small angle approximation

$$
x=\frac{f m \lambda}{d}
$$

Note that this example only describes the diffraction orders in one dimension. The CTIS grating is actually a $2 \mathrm{D}$ grating so there will be a two dimensional array of diffraction orders with coordinates, $(\mathrm{x}, \mathrm{y})$, given by:

$$
x=\frac{m_{k} \lambda f}{d_{x}} \text { and } y=\frac{m_{\ell} \lambda f}{d_{y}}
$$

Where $\mathrm{m}_{\mathrm{k}}$ is the integer order number in the $\mathrm{x}$ direction, $\mathrm{m}_{\mathrm{l}}$ is the order number in the $\mathrm{y}$ direction, $\mathrm{d}_{\mathrm{x}}$ is the grating period in the $\mathrm{x}$ direction, and $\mathrm{d}_{\mathrm{y}}$ is the grating period in the $\mathrm{y}$ direction. This description of the location of the orders is an important result that will be seen again when using Fourier optics to analyze the performance of the grating in chapter 4.

So this provides a description of the relative location of each of the diffraction orders for a point in the scene. Now instead of considering a scene that just consists of a single spectrally broadband point at the center of the field stop, let us now consider a purely monochromatic scene that fills the entire field stop. We already know that the size of the zero order image will be given by equation (3.2).

Zero Order Image Size $=\mathrm{h}\left(\frac{f_{\text {reim }}}{f_{\text {col }}}\right)$

Because this scene is only composed of a single wavelength, the CTIS will effectively replicate that monochromatic image in each of the diffraction orders. This image replication occurs because, to first order, the relative physical displacement for each of the diffraction orders will be the same for every point in the scene. If the location of the corresponding diffraction order is shifted by the same amount for all the points in the scene, the effect is that the entire image is replicated and offset by that same amount. These replicated images, however, will have some attenuation given by the diffraction efficiency of that particular order for the corresponding wavelength. The size of each replicated image will be the same as the zero order image size and each replicated image will be centered around the coordinates (x,y) given by equation (3.8):

$x=m_{k} \lambda f / d_{x}$ and $y=m_{l} \lambda f / d_{y}$

If the scene were to be composed of multiple wavelengths, the monochromatic images would be replicated for each wavelength component, with each wavelength component centered on a different location. As illustrated in figure 3.5, the diffraction orders in a CTIS system are composed of overlapping monochromatic images corresponding to the spectral components of the scene. It is the overlap of the monochromatic images that cause the spatial/spectral 
multiplexing in the recorded data, and will determine the size and location of each diffraction order.
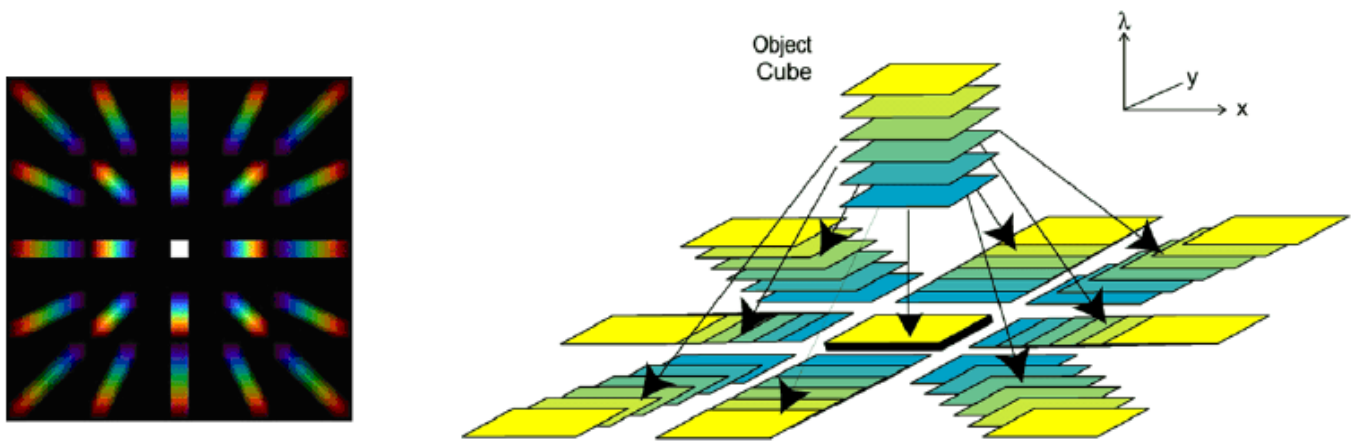

Figure 3.5: (left) Example of recorded image of a broadband white light source recorded by a visible CTIS system. (right) Illustration of the overlapping monochromatic images in the diffraction orders

We now know what parameters control the size and location of the various diffraction orders, and can begin to investigate how these parameters can be selected to prevent overlap between the orders to decrease the multiplexing in the system. Consider the case of a $3 \times 3$ CTIS diffraction pattern. In order to avoid overlap between the diffraction orders the designer must ensure that the displacement for the lowest wavelength in the +1 horizontal order is greater than the size of the zero order, as seen in figure 3.6.
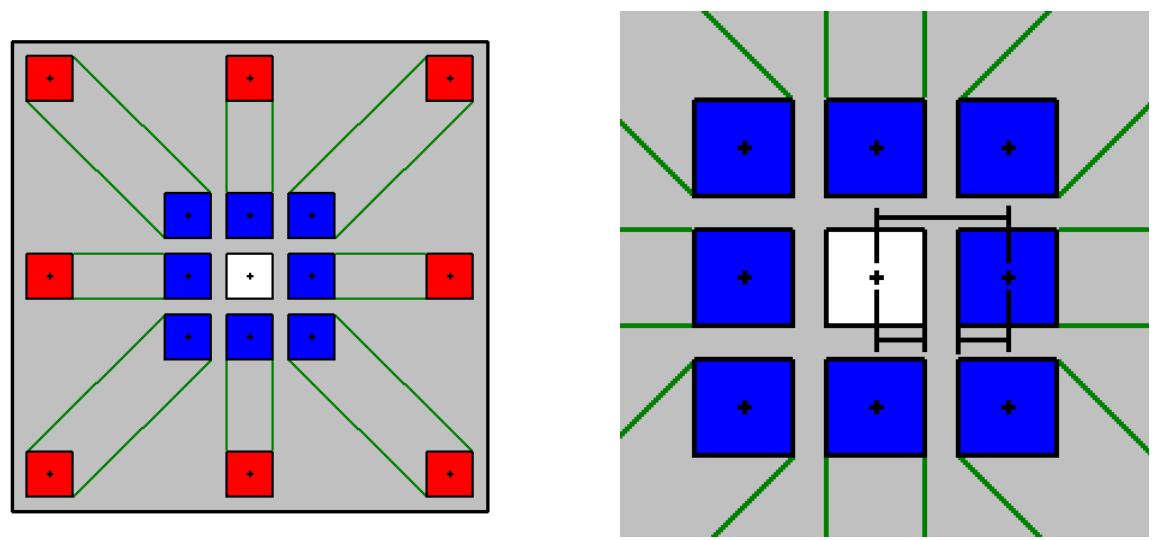

Figure 3.6: (left) Illustration of a 3x3 CTIS diffraction pattern with no overlap between the orders. The highest red wavelengths form images near the edge of the FPA, while the lowest blue wavelength forms replicated monochromatic images just outside of the white zero order. (right) Zoomed in portion of illustration showing no overlap between the central panchromatic zero order and the outer +1 diffraction orders. Note that for the lowest specified wavelength (blue) the displacement of the center of the monochromatic replicated blue image in the +1 order is greater than the size of the white zero order. In the figure, the length of each of the two bars below the zero order are each half the size of the zero order (combined equaling the size of the zero order), while the bar above the zero order representing the displacement of the lowest wavelength for the +1 order is shown to be greater than the size of the zero order. 
In the case of a $5 \times 5$ CTIS diffraction pattern, in order to avoid overlap between the diffraction orders, the designer must ensure that the difference between the displacement of the low wavelength end of the +2 horizontal diffraction and the high wavelength end of the +1 horizontal order is greater than the size of the zero order, as illustrated in figure 3.7.
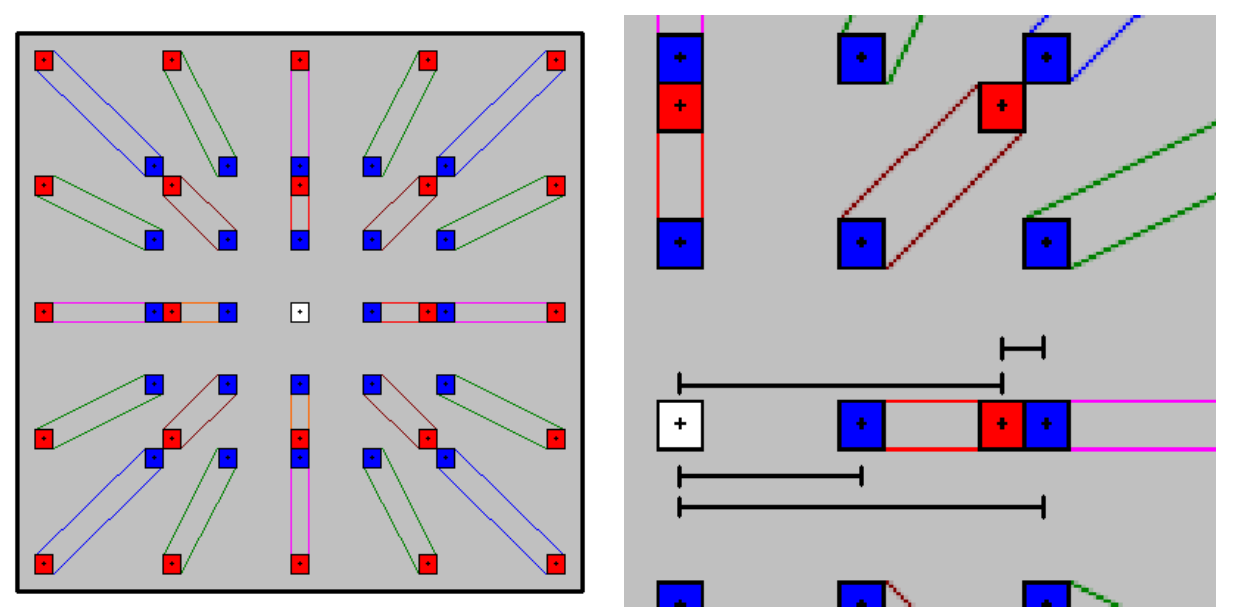

Figure 3.7: (left) Illustration of a 5x5 CTIS diffraction pattern with no overlap between any of the orders. (right) Zoomed in portion of illustration showing that the displacement of the lowest wavelength blue image in the +2 order minus the displacement of the highest wavelength red image in the +1 diffraction order must be greater than the size of the zero order in order to avoid overlap between the +1 and +2 diffraction orders. This will limit the allowable spectral range between the low wavelength and high wavelength if there is to be no overlap in a $5 \times 5$ diffraction pattern. Provided this condition is met there will also be no overlap between the central zero order and the +1 orders. Since the small angle approximation was applied, the displacement for the lowest wavelength in the +2 order will be twice the displacement of the lowest wavelength in the +1 order.

To prevent order overlap in a CTIS diffraction pattern that consists of only the outermost orders of a $5 \times 5$ pattern, as shown in figure 3.8, the designer only needs to consider the location and size of the monochromatic images corresponding to the lowest wavelength as well as the orientation angles for the diffraction orders.
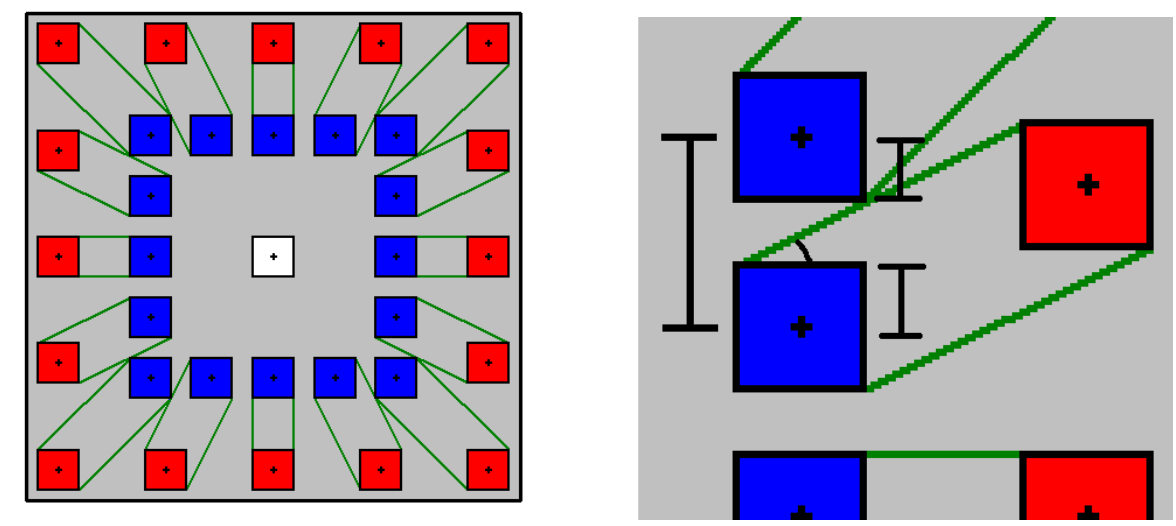

Figure 3.8: (left) Illustration of an outer 5x5 CTIS diffraction pattern with no overlap between any of the orders. (right) Zoomed in portion illustrating where the overlap must be avoided. 
Table 1 on the following page specifies the relative values for the physical field stop size, $\mathrm{h}$; grating period, d; and collimating lens focal length, $\mathrm{f}_{\text {col }}$, needed in order to prevent overlapping diffraction orders for a variety of common CTIS diffraction order patterns. These conditions are based on the calculated displacement positions, the calculated zero order image sizes, and the necessary requirements to avoid overlap for each diffraction pattern similar to what was shown in the preceding figures.

There are a few things to note in regards to the conditions for no overlap presented in Table 1. In many standard grating applications, this condition of eliminating spectral overlap for a given diffraction order is given by the free spectral range. The free spectral range is defined as the range of wavelengths, $\Delta \lambda$, in a given diffraction order, $m$, for which superposition of light from adjacent orders does not occur and is given by the following equation.

$\Delta \lambda=\lambda_{\text {low }} / \mathrm{m}$

In other words, the diffraction angle in the $\mathrm{m}^{\text {th }}$ diffraction order for light with a higher wavelength of $\lambda_{\text {high }}=\lambda_{\text {low }}+\Delta \lambda$ will be the same diffraction angle as light with a wavelength of $\lambda_{\text {low }}$ in the $(\mathrm{m}+1)$ diffraction order and there will be no other overlap in the $\mathrm{m}^{\text {th }}$ order. In CTIS systems, the conditions to avoid overlap are not quite as simple because the zero order is not a single point. The zero order has some finite size, which will limit the selected wavelengths and the wavelength region depending upon the CTIS system parameters. For example, in a $3 \times 3$ CTIS diffraction pattern the only region where overlap between orders needs to be eliminated is between the 0 order and the +1 (or alternatively -1 ) diffraction orders. In terms of the highest wavelength allowed to prevent overlap between orders, traditionally given by the free spectral range, there is no limit on the high wavelength in this case. The high wavelength can be arbitrarily high and still avoid overlap because the design of the CGH grating can be made to have no light sent into the +2 order or any of the higher orders that would overlap with the +1 order. The only limit will be on the lowest wavelength in order to prevent the physical size of the zero order from overlapping the spatial position of the +1 order for the lowest wavelength.

However there are other CTIS diffraction patterns where the traditional free spectral range definition will be a component of the conditions to eliminate overlap for the CTIS pattern. The traditional free spectral range limits on the high and low wavelengths can be expressed as follows.

$\lambda_{\text {high }}=\lambda_{\text {low }}+\Delta \lambda=\lambda_{\text {low }}((\mathrm{m}+1) / \mathrm{m}) \Rightarrow(\mathrm{m}+1) \lambda_{\text {low }}-(\mathrm{m}) \lambda_{\text {high }}=0$

Looking at the conditions needed to avoid overlap for the $5 \times 5$ CTIS diffraction pattern and the $7 \times 7$ CTIS diffraction pattern, this same term of $(m+1) \lambda_{\text {low }}-(m) \lambda_{\text {high }}$ appears in the numerator of the left hand side of the inequality. In the $5 \times 5$ pattern $\mathrm{m}=1$ since overlap must be eliminated between the +1 orders and the +2 orders. Similarly $m=2$ for the $7 \times 7$ pattern, to prevent overlap between the +2 and +3 orders. Again however, the no overlap condition for CTIS is more strict than the standard free spectral range definition due to the finite size of the zero order. The maximum range of wavelengths is no longer just determined by $(\mathrm{m}+1) \lambda_{\text {low }}-$ ( $\mathrm{m}$ ) $\lambda_{\text {high }}$ equaling zero, it will be smaller since the same must now be further limited to being greater than or equal to the grating period times a factor related to the size of the zero order. 
Table of CTIS diffraction patterns and the required condition to prevent overlap of the selected diffraction orders

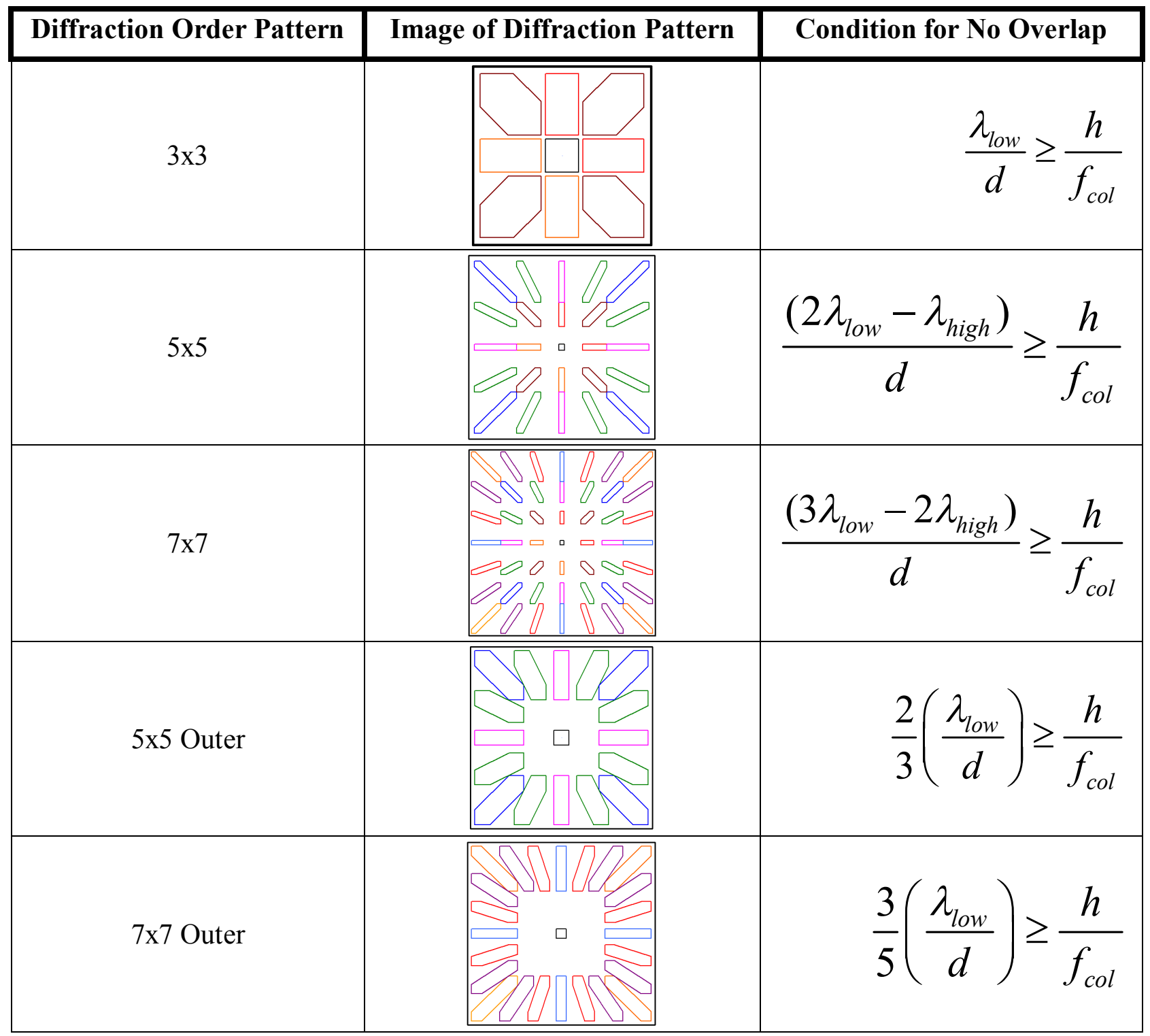

Table 1: Comparison of different CTIS diffraction order patterns and the required condition for the CTIS system parameters to prevent overlap of the selected orders in the pattern. Note that in all of the stated values for the no overlap conditions, the small angle approximation was made in regards to the grating diffraction angle and that the grating period, $d$, is assumed to be the same in both the $\mathrm{x}$ and $\mathrm{y}$ directions. 
Based on the conditions provided, there is no way to avoid overlap in a $5 \times 5$ design if the selected highest wavelength, $\lambda_{\text {high }}$, is greater than two times the lowest wavelength, $\lambda_{\text {low }}$. The higher wavelengths of the +1 order will always spill over into the lower wavelengths of the +2 order. The range of wavelengths required to avoid overlap is even tighter for a $7 \times 7$ design. In that case $\lambda_{\text {high }}$ can at most be 1.5 times $\lambda_{\text {low }}$ to avoid overlap. This means that you can not have a $7 \times 7$ CTIS design operating from $400 \mathrm{~nm}$ to $700 \mathrm{~nm}$ without some overlap in the orders. It also means that you can not have a 7x7 CTIS operating from 8 microns to 12 microns without some overlap in the orders. In the LWIR example, the highest wavelength is exactly 1.5 times the lowest wavelength resulting in the left hand side of the inequality presented in the table equaling zero. Since the field stop size will always have some finite, nonzero, height it will be impossible for the right hand side of the inequality to be less than or equal to the left making it impossible to avoid overlap in a 7x7 CTIS operating from 8 to 12 microns. One way to mitigate these problems is to design the CGH to only send light into the outer orders. As you can see in the requirements for the $5 \times 5$ outer design, and the $7 \times 7$ outer design, there are no limits to the values of $\lambda_{\text {high. }}$

Note that this analysis has only looked at the conditions to avoid overlap in the orders and has not examined which pattern would produce better reconstruction results. The "outer" diffraction order designs can have a number of advantages including eliminating some of the redundant information contained in some of the inner orders, increase in the recorded image signal, and increasing the speed of reconstruction. While other designs such as the asymmetric quad pattern can provide higher spatial and spectral sampling, the limited number of projections may not provide any increase in reconstructed resolution depending upon the application.

Step four in the design process requires determining the focal length of the reimaging lens of the system. The initial parameter values of the system have already been selected to prevent any overlap in the diffraction orders; however, the overall size of this non overlapping pattern will be determined by the reimaging lens. By changing the focal length of the reimaging, the minification/magnification of the entire CTIS image can be adjusted, see figure 3.9, such that the resulting image fits on the focal plane array, as illustrated in figure 3.10.

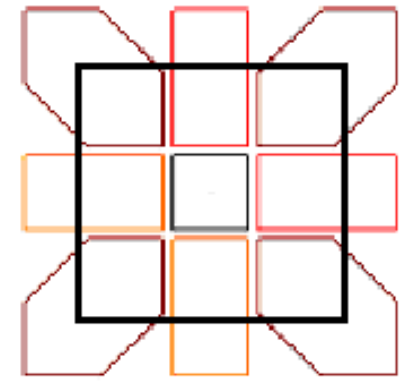

(a)

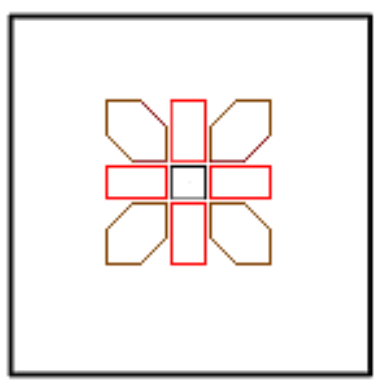

(b)

Figure 3.9: (a) Example of condition where the focal length of the reimaging lens is too high.

The produced CTIS image has no overlap between the orders, but is larger than the focal plane.

(b) Example of condition where the focal length of the reimaging lens is too low. The produced

CTIS image has no overlap between the orders, but is very small on the focal plane. In both cases the focal length of the reimaging lens must be adjusted. 
Specifically, the reimaging lens focal length will be constrained by the following relationship:

$$
f_{\text {reim }} \leq \frac{\text { ArraySize }}{\left(\frac{2 M \lambda_{\text {high }}}{d}+\frac{h}{f_{c o l}}\right)}
$$

Where the value $\mathrm{M}$ is related to the order number of the diffraction pattern, which is listed below for a variety of patterns, and ArraySize is the physical length of the smallest dimension of the focal plane used to record the CTIS image.

$\mathrm{M}=1$ for $3 \times 3$

$M=2$ for $5 \times 5$

$\mathrm{M}=3$ for $7 \mathrm{x} 7$

This equation is the result of constraining the size of the zero order image plus two times the grating's displacement of the highest wavelength components, both of which vary depending upon the reimaging lens focal length, to be less than the shortest dimension of the focal plane array size.

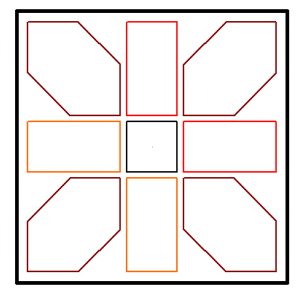

Figure 3.10: With the reimaging lens focal length changed appropriately, the CTIS diffraction pattern now fits on the FPA.

Step five in the design process involves scaling some of the initially selected values to ensure that the overall system meets certain manufacturing and performance requirements for the grating, system field of view requirements, allowing the system to work with only a limited availability of lens focal lengths, etc. Since the initial values were carefully selected to prevent overlapping diffraction orders as well as making the entire CTIS image fit onto the focal plane, any changes made to these values must be carefully done so as not to undo this previous work. In other words, any changes made to the initial parameter values must produce the same CTIS image as before. All of the diffraction orders should be in the same locations and the size of the diffraction orders will also need to be the same. This is a challenge because all of the system parameters are interrelated and have various effects on the resulting image.

In order to produce the same CTIS image as what is given by the initially selected values, you must follow the following rules. 
- RULE 1: If either the grating period or the focal length of the reimaging lens needs to be changed from their initial value, the other parameter (either the grating period or reimaging lens focal length) must also be changed by the same multiplicative factor.

- RULE 2: Any changes to the reimaging lens focal length, field stop size, or collimating lens focal length from their initial values must be made such that the ratio of the focal length of the collimating lens to the product of the field stop size and reimaging lens focal length does not change.

It is important to note that there are multiple sets of CTIS component values that will produce the same image. Let's examine how these sets of parameters are related and how the rules above are derived. In order for the locations of the CTIS diffraction orders to be the same, the physical displacement of each diffraction order, given by the following equation, must also be the same.

$$
x=\frac{f_{\text {reim }} m \lambda}{d}
$$

From this equation we know that the ratio of the focal length of the reimaging lens to the grating period determines where all of the diffraction orders will be spatially located on the focal plane for each wavelength. So long as the ratio of the reimaging lens focal length and the grating period remains the same, there will be no difference in where the center of the monochromatic images in the diffraction orders are mapped to on the focal plane. When making changes to either of these values, the newly selected values of the reimaging lens focal length and grating period, which are indicated using primes in the following equations, must be increased or decreased from the initial values, indicated without primes, by the same constant factor in order for the relative location of the diffraction orders to remain the same.

$\mathrm{f}_{\text {reim }}{ }^{\prime}=\mathrm{k}_{1} * \mathrm{f}_{\text {reim }}$

$\mathrm{d}^{\prime}=\mathrm{k}_{1} * \mathrm{~d}$

This is especially useful because there are some manufacturing and performance constraints on how small the grating period can be, which are related to the highest wavelength of the selected waveband. In the design of the grating, which is covered in the following chapter, the designer will specify a particular number of phase elements within the grating period. It is recommended that each of these phase elements have a physical size that is at least twice as big as the longest wavelength in order for the mathematical assumptions on how the grating will perform to remain true. In practice, there is also a limit on the minimum number of phase elements that can be used in the grating period. If too few phase elements are used, it can be difficult to design a grating with sufficient performance. In such cases, creating a design which uses more phase elements may improve performance. Based on the specifics of the grating design, the optical designer may need to revisit the initially selected values and increase the grating period of the system to meet the minimum size of the grating period, or decrease the initial value of the grating period, allowing more periods of the grating to be seen by the light resulting in a tightening of the point spread function in each of the diffraction orders. (Periodicity of the grating breeds punctility in the psf.) 
This scaling condition allows the location of the recorded diffraction orders to be the same, however it does not guarantee that the resulting entire CTIS image for the system will be same. Some of the remaining optical parameters must be changed such that the size of the recorded diffraction orders is the same as well. Going back to the previous equation relating the size of the zero order image to the size of the field stop aperture:

zero order image size $=h\left(f_{\text {reim }} / f_{\text {col }}\right)$

We know that the monochromatic image replicated in each diffraction order for each wavelength component will have this same size as well. In order for the physical size of the recorded diffraction orders to remain the same one of the following three conditions must be met (primes indicate new values with non-primes as the initial values):

1. Both reimaging and collimating lens focal lengths are scaled by the same amount.

$$
\begin{aligned}
& \mathrm{f}_{\text {reim }}{ }^{\prime}=\mathrm{k}_{2} * \mathrm{f}_{\text {reim }} \\
& \mathrm{f}_{\text {col }}{ }^{\prime}=\mathrm{k}_{2} * \mathrm{f}_{\text {col }}
\end{aligned}
$$

2. Both the field stop size and the collimating lens focal length are scaled by the same amount

$$
\begin{aligned}
& \mathrm{h}^{\prime}=\mathrm{k}_{3} * \mathrm{~h} \\
& \mathrm{f}_{\text {col }}=\mathrm{k}_{3} * \mathrm{f}_{\text {col }}
\end{aligned}
$$

3. The product of the newly selected physical field stop size and new reimaging lens focal length relative to their initial values must be scaled by the same amount as the newly selected collimating lens focal length relative to its initial value

$$
\begin{aligned}
& \left(\mathrm{h}^{\prime} * \mathrm{f}_{\text {reim }}{ }^{\prime}\right)=\mathrm{k}_{4} *\left(\mathrm{~h}^{*} \mathrm{f}_{\text {reim }}\right) \\
& \mathrm{f}_{\text {col }}=\mathrm{k}_{4} * \mathrm{f}_{\text {col }}
\end{aligned}
$$

In the first case, recall that the magnification of the field stop on the focal plane is given by the ratio of the reimaging lens focal length to the collimating lens focal length. If both of these lens focal lengths are scaled by the same amount, the magnification of the field stop is unchanged. When combining this condition, which keeps the size of the orders the same, with the rule required to keep the location of the diffraction orders the same as well, we see that the grating period must also be scaled by the same factor. So if the optical designer previously needed to change the grating period, in order to keep the CTIS image fitting on the focal plane both the reimaging and collimating lens focal lengths must be changed by the same amount. Changing only these parameters will allow the designer to keep the field stop size, and thus the field of view of the system, unchanged.

The second case, is a useful way of being able to alter the field of view of the system without having to change any of the back end components from their initial values. Again, the ratio of the reimaging lens focal length to the collimating lens focal length determines the magnification of the field stop. If the collimating lens is changed by a factor of $\mathrm{K}$, the magnification of the 
field stop is decreased by a factor of $(1 / \mathrm{K})$. In order to compensate for this change in magnification, we must increase the size of the field stop by a factor of $\mathrm{K}$. The net effect, provided you are using the same objective lens, is that only the field of view of the system is changed.

The focal length of the objective lens and the physical size of the field stop aperture determine the field of view of the system. To first order, the field stop provides a sharp cutoff between the unvignetted and fully vignetted rays, limiting the field of view of the system, as shown in figure 3.11 .
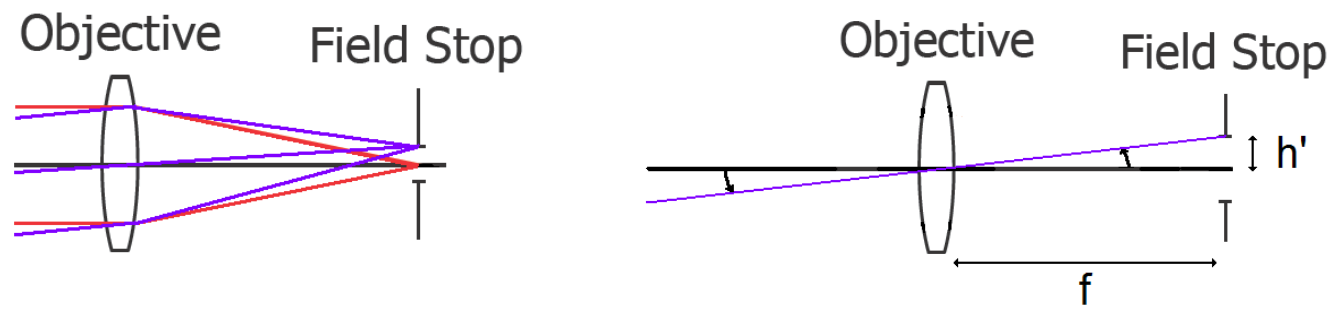

Figure 3.11: Illustrations showing how the focal length of the objective in combination with the size of the field stop aperture will limit the light passing through the system and will determine the field of view of the system.

If the designer has a strict field of view requirement, you can calculated the needed objective lens focal length using the following equation.

$$
f_{\text {objective }}=\frac{h}{2 \times \tan \left(\frac{\theta_{\text {fullFOV }}}{2}\right)} \approx \frac{h}{\theta_{\text {fullFOV }}}
$$

In the third case, all of the parameters of the system are changed from their initial values. To keep the CTIS image the same, the grating and reimaging lens must be scaled by the same constant factor, $\mathrm{k}_{1}$. The collimating lens must be scaled from its initial value by a constant factor of $\mathrm{k}_{4}$, and finally the field stop size must be scaled from its initial value by a factor of $\left(\mathrm{k}_{4} / \mathrm{k}_{1}\right)$. If there is a limited selection of available focal length lenses, this is a useful way of keeping the CTIS image you want. The designer can scale the lenses to the needed values, but since he or she has full control over the grating period and field stop size, those parameters can be changed to work with those selected lenses.

Once a new set of optical component values have been selected, check to make sure that these values meet all of your requirements for the system. If all of the requirements are not met, it may be necessary to follow the same rules and iterate the component selection again, or you may need to select different initial values based on the initial given inequalities and continue the design from there. As an example, the steps taken when designing a long wavelength infrared CTIS are presented below.

System Requirements: 
Diffraction Order Pattern: $5 \times 5$ diffraction pattern

Wavelength Range: 8 micron -12 micron

Focal Plane Array: 640 x 480 Silicon Microbolometer

Pixel Pitch: 25 microns

Reimaging lens can not be changed. The lens and focal plane are an integrated camera unit, with the reimaging lens having a focal length of $62 \mathrm{~mm}$.

Goal for the system is to have a 5 degree field of view, with a minimum allowed field of view of 2 degrees.

Commercial off the shelf LWIR camera lenses are to be used, of which there is a limited selection of focal lengths.

Step 1) Diffraction order pattern will be a $5 \times 5$ design.

Step 2) Wavelength range will be from 8 microns to 12 microns.

Step 3) Select grating period, field stop size, and collimating lens focal length.

To avoid overlap of the diffraction orders for a $5 \times 5$ design, the following inequality must be met:

$$
\frac{\left(2 \lambda_{\text {low }}-\lambda_{\text {high }}\right)}{d} \geq \frac{h}{f_{\text {col }}}
$$

$\lambda_{\text {low }}$ will be 8 microns. $\lambda_{\text {high }}$ will be 12 microns. The initial grating period was arbitrarily selected to have a value of 100 microns.

$\mathrm{d}=100$ micron

The values for the field stop size and collimating lens are chosen so that the diffraction orders are as close as possible without overlapping. In this case both sides of the expression are equal.

The following selected values were for the field stop size and collimating lens focal length seemed like reasonable values that met that condition.

$\mathrm{h}=4 \mathrm{~mm}$

fcol $=100 \mathrm{~mm}$

You can decrease $h$ or increase fcol to increase the separation between the orders

Step 4) Calculate reimaging lens focal length.

$$
f_{\text {reim }} \leq \frac{\text { ArraySize }}{\left(\frac{2 M \lambda_{\text {high }}}{d}+\frac{h}{f_{\text {col }}}\right)}
$$

M equals 2 for a $5 \times 5$ pattern. The design will fit on a $480 \times 480$ section of the focal plane with a 
pixel pitch of 25 microns, corresponding to an array size of $12.0 \mathrm{~mm}$.

The focal length of the reimaging lens must be less than or equal to $23.07 \mathrm{~mm}$.

Step 5) The initial values for the system have been selected, and we have encountered the first conflict of this design. The focal length of the reimaging lens is required to have a value of $62 \mathrm{~mm}$, it is a fixed component connected to the focal plane as part of a single camera system. We can scale the initial value of the reimaging focal length of to have a maximum value of $62 \mathrm{~mm}$ by multiplying by a factor of 2.687 . Since the reimaging lens focal length is changed from its initial value, in order to keep the orders in the same physical location, the grating period must be scaled by the same amount. In this case the grating period will be increased by a factor of 2.687 from an initial value of 100 microns to a value of 268.7 microns.

After working on the specifics of the grating design, the optical designer has discovered that the grating must contain at least 12 phase elements in each direction of the square grating period, in order for the grating to meet its performance requirements. The physical size of each phase element is suggested to be at least twice the size of the longest wavelength. In this case, the longest wavelength is 12 microns, meaning that the smallest grating period allowed with 12 phase elements in each direction would be 288 microns in length. The current grating period value of 268.7 microns is too small.

We can scale the initial grating period from 100 microns to 288 microns with a factor of 2.88 . Since the grating period has changed, the reimaging lens focal length must also be changed by the same amount. The focal length of the reimaging lens goes from needing to be less than or equal to $23.07 \mathrm{~mm}$ to needing to be less than or equal to $66.44 \mathrm{~mm}$. The fixed reimaging focal length value of $62 \mathrm{~mm}$ meets this requirement.

In an attempt to prevent changing the initially selected $100 \mathrm{~mm}$ focal length collimating lens, we can determine what field stop size is needed by using the third condition for keeping the diffraction order sizes the same size. Since the reimaging lens was scaled by a factor of 2.88 and the collimating lens is unchanged (scaled by a factor of 1 ), the field stop size must be scaled by a factor of $(1 / 2.88)$ changing from an initial value of $4.0 \mathrm{~mm}$ to $1.39 \mathrm{~mm}$.

To try and meet the final remaining field of view requirement, the designer can select the smallest available focal length for the objective lens, which in this case is $50 \mathrm{~mm}$ due to the limited selection of LWIR lenses. When used in combination with a $1.39 \mathrm{~mm}$ field stop the resulting field of view for the system is 1.6 degrees, below the minimum required amount of 2.0 degrees. There is a simple fix for this. By using the second condition and scaling the collimating lens and the field stop size, provided that a collimating lens is available with a focal length greater than $100 \mathrm{~mm}$, we can increase the field of view of the system without having to change any other components. The maximum focal length of the available commercial off the shelf LWIR camera lenses is $150 \mathrm{~mm}$, an increase by a factor of 1.5 . Using this lens, we can increase the field stop size by the same factor of 1.5 from a value of $1.39 \mathrm{~mm}$ to $2.08 \mathrm{~mm}$. Having a $2.08 \mathrm{~mm}$ field stop with a $50 \mathrm{~mm}$ objective lens results in a 2.29 degree system field of view, which meets the stated requirement. 
Ultimately, the size of the field stop is decreased slightly to a value of $2.0 \mathrm{~mm}$ in order to provide a little extra spacing between the diffraction orders. Even though the design does not overlap to first order designs, the edges of the orders will be a bit larger due to size of the psf.

Now that the first order optical properties of the system have been selected to create an appropriately sized CTIS image diffraction pattern that can be recorded by the focal plane, the designer must now specify the phase element details of the disperser, which will try to send light into the desired diffraction orders. The details on the CTIS disperser design are presented in the following chapter. 


\section{REVIEW OF HOW TO COMPLETE FIRST ORDER CTIS OPTICAL DESIGN:}

1) Select Diffraction Order Pattern (examples: $3 \times 3,5 \times 5$, outer $7 \times 7$, etc.)

2) Specify Wavelength Range (examples: $400 \mathrm{~nm}$ to $700 \mathrm{~nm}, 8$ microns to 12 microns, etc.)

3) Use table to specify initial values for field stop size, $h$, grating period, $d$, and collimating lens focal length, $f_{c o l}$, to avoid overlap in the diffraction orders for selected diffraction pattern.

\begin{tabular}{|c|r|}
\hline $3 \times 3$ & $\frac{\lambda_{\text {low }}}{d} \geq \frac{h}{f_{\text {col }}}$ \\
\hline $5 \times 5$ & $\frac{\left(2 \lambda_{\text {low }}-\lambda_{\text {high }}\right)}{d} \geq \frac{h}{f_{\text {col }}}$ \\
\hline $7 \times 7$ & $\frac{\left(3 \lambda_{\text {low }}-2 \lambda_{\text {high }}\right)}{d} \geq \frac{h}{f_{\text {col }}}$ \\
\hline $5 \times 5$ Outer & $\frac{2}{3}\left(\frac{\lambda_{\text {low }}}{d}\right) \geq \frac{h}{f_{\text {col }}}$ \\
\hline $7 \times 7$ Outer & $\frac{3}{5}\left(\frac{\lambda_{\text {low }}}{d}\right) \geq \frac{h}{f_{\text {col }}}$ \\
\hline
\end{tabular}

4) Calculate reimaging lens focal length to get the pattern to fit on the focal plane

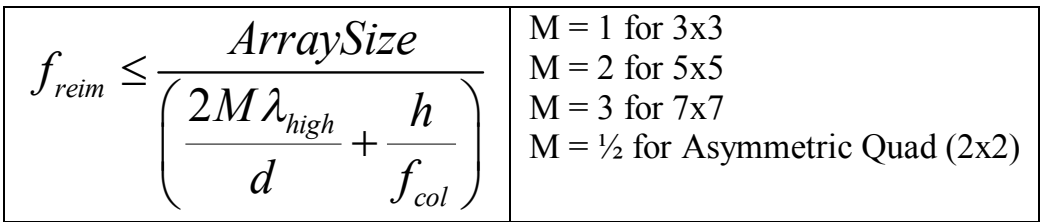

5) Certain values will likely need to be changed to meet field of view requirements, manufacturing and performance requirements for the grating, limited selection of lens focal lengths, etc. Follow the specified rules to scale particular optical parameter values, whose changes will produce the same CTIS image filling the focal plane.

- RULE 1: If either the grating period or the focal length of the reimaging lens needs to be changed from their initial values, the other parameter (either the grating period or reimaging lens focal length) must also be changed by the same multiplicative factor.

- RULE 2: Any changes to the reimaging lens focal length, field stop size, or collimating lens focal length from their initial values must be made such that the ratio of the focal length of the collimating lens to the product of the field stop size and reimaging lens focal length does not change.

Calculate focal length of the objective lens based on your field of view requirement.

$$
f_{\text {objective }}=\frac{h}{2 \times \tan \left(\frac{\theta_{\text {fullFOV }}}{2}\right)} \approx \frac{h}{\theta_{\text {fullFOV }}}
$$




\section{CHAPTER 4: CTIS CGH DESIGN}

\subsection{CTIS CGH Disperser Overview}

The CGH disperser is considered to be the most important element of the entire CTIS system. It is the one component that controls how we measure the spectral and spatial information from the scene, and because of this it is sometimes referred to as the "workhorse" optical element of a CTIS. While all of the other components can often be obtained as commercial off the shelf parts, the CGH disperser is a specialized component that will need to be custom manufactured. This custom part can simply be described as a specialized blazed two-dimensional diffraction grating.

Just as in a regular one-dimensional diffraction grating, illustrated in figure 4.1, the grating is composed of a periodic structure. Incident light hitting that periodic structure is separated into different parts, forming various diffraction orders. Some of that incident light travels straight through the grating forming the panchromatic zero diffraction order. While in the outer diffraction orders, the different wavelengths of the incident light are sent in different directions, separating out the spectral components of the incident light in each order. By changing the shape of the periodic structure of the grating, the diffraction grating can be "blazed" to control how much light is sent into each of the diffraction orders.
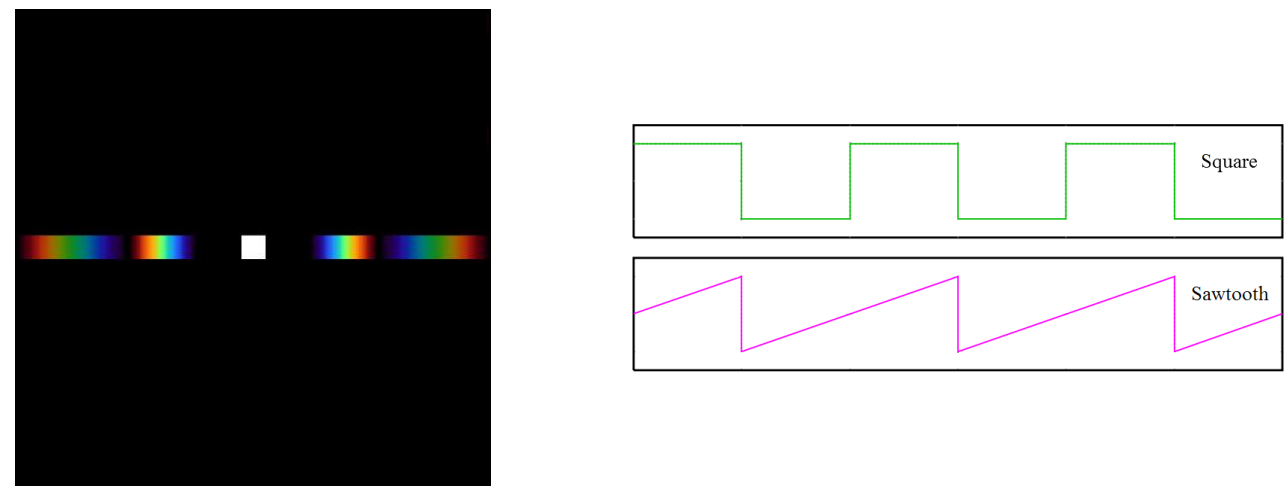

Figure 4.1: (left) Illustration of the various diffraction orders produced by a one-dimensional diffraction grating with broadband visible light input. (right) Examples of possible blazed grating profiles. These periodic structures are repeated in one dimension of the grating surface.

The CTIS CGH is basically a blazed two-dimensional diffraction grating. It is composed of a specialized two-dimensional periodic structure whose shape has been specifically designed to only send light into a two dimensional array of diffraction orders, as shown in figure 4.2. 

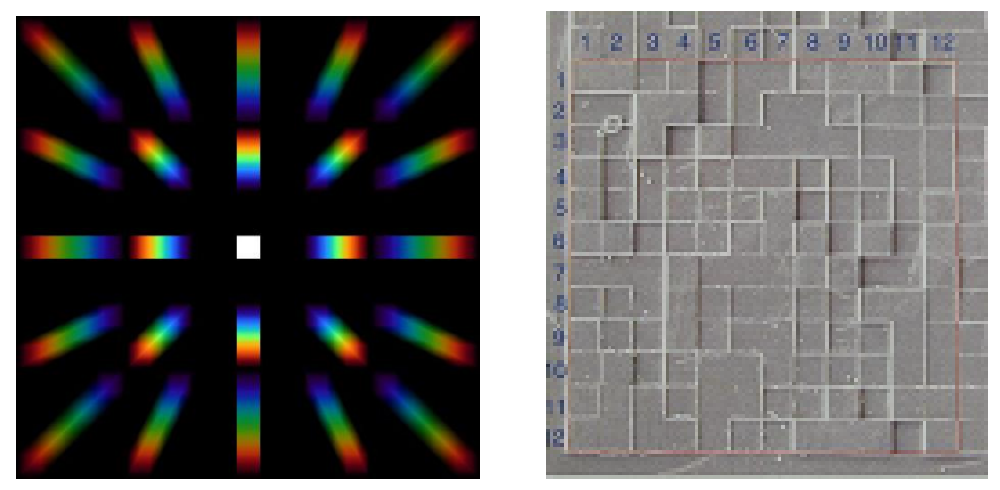

Figure 4.2: (left) Illustration of the various diffraction orders produced by a two-dimensional diffraction grating with broadband visible light input. (right) Photo of a single grating period

from a CTIS CGH. This two-dimensional structure consisting of various height levels is periodically repeated in both the $\mathrm{x}$ and $\mathrm{y}$ dimensions of the two-dimensional CGH grating.

Changes to the surface profile, or the height of the surface features, will alter the amount of light sent into each diffraction order.

\subsection{Design of CTIS CGH Disperser}

The primary challenges of designing the computer generated hologram for a CTIS disperser are:

- getting the light where the designer wants it to be on the focal plane

- having the correct diffraction efficiency/amount of light in each diffraction order

- and simultaneously managing both of these tasks for all the wavelengths in the specified range of the CTIS with the fixed CGH.

These issues will be dependent upon two properties of the CTIS CGH diffraction grating: the length of the $\mathrm{CGH}$ grating period in each dimension and the phase profile of each period of the CGH. It has already been shown in chapter 3 how the grating period, in combination with the other optics in the system, determine where the diffraction orders for each wavelength will be located on the focal plane. The remaining challenge in the design of the CTIS disperser is to select the phase profile/shape for the grating period that produces the desired diffraction efficiency for each diffraction order and is able to do so for all of the specified wavelengths in the region.

In an amplitude grating, the grating's structure will periodically absorb light across the surface of the optic, but this absorption of the incident light greatly reduces the possible diffraction efficiencies of the optic. A phase grating, however, just imposes a periodic phase variation across the incident wavefront of light, without absorbing any of the incident light. The resulting diffraction efficiencies for the phase grating can be significantly higher, producing a more light efficient optical component. The CTIS disperser should be as light efficient as possible, so the first CTIS disperser, made by Descour ${ }^{9}$, used a combination of three cosine phase gratings each rotated 60 degrees relative to the others to form a hexagonal diffraction order pattern. Volin later improved upon this design by developing an algorithm for the design of phase computer generated hologram $(\mathrm{CGH})$ that allowed for the $\mathrm{CGH}$ to have polychromatic operation. ${ }^{10}$ Volin's technique allowed for a greater control of the diffraction efficiencies of the orders and 
allowed for an increased number of diffraction order projections to be produced. The CGH disperser for the LWIR CTIS will ultimately be a phase grating created by using the polychromatic technique.

Determining an appropriate phase profile for the CTIS CGH will be investigated with the following steps. First, we will describe how the diffraction efficiencies of each diffraction order can be calculated as a function of wavelength for a given CGH phase profile. This calculation will serve as the tool used for evaluating the performance of various phase profile designs. The next step is to use this evaluation tool to try and design a phase profile that achieves the best performance. Algorithms developed for computer generated holography use this evaluation method in a systematic way to design the phase profile of a CGH that produces a given desired irradiance pattern. While many of these algorithms are limited to producing specific irradiance patterns for a single wavelength, the Volin polychromatic CGH design algorithm, briefly described above, overcomes this limitation and will be the algorithm used in the CTIS CGH design. Details on this polychromatic design algorithm will be presented. The final step is to determine the appropriate goal irradiance pattern for a CTIS imaging spectrometer system that the algorithm will design the phase profile to produce. This problem is approached in two ways: for a given CTIS system with a specified diffraction pattern, what are the optimum diffraction efficiencies for each diffraction order, and which CTIS diffraction pattern is the best for the system? Before discussing these later steps, let us first cover the mathematical description and operation of a periodic phase grating and determine how the diffraction efficiencies for a particular phase profile can be calculated.

\subsection{Calculating Diffraction Efficiencies for a Particular Phase Profile and How the Phase Pattern of the CGH Relates to The Irradiance at the Image Plane}

In general, this periodic phase profile of the CTIS disperser/grating is described as consisting of a discrete number of phase elements, or "phasels." The phase delay for each phase element will depend upon the physical thickness of the element as well as the index of refraction of the material for the wavelength $\lambda$, and is given by:

$$
\Phi_{\lambda}=\frac{2 \pi\left(n_{\lambda}-1\right) d}{\lambda}
$$

Where $\Phi_{\lambda}$ is the phase delay of the element for the wavelength $\lambda, \mathrm{n}_{\lambda}$ is the material index of refraction at the wavelength $\lambda$, and $d$ is the physical thickness of the material. In this analysis, the phase profile across the grating period will not be described as a continuous function. It will be represented by a large number of discrete phase elements, or phasels, in the same way that pixels are used to display a digital image.

The transmission function for a single phase element will not affect the amplitude of the incident light. It will only impart a phase delay depending upon the physical thickness and index of refraction of the material. Each phasel is described as having different physical dimensions in the $\mathrm{x}$ and $\mathrm{y}$ axes. If the physical length of the phasel in the $\mathrm{x}$ dimension at the CGH plane is given by a length $\xi_{\mathrm{c}}$ and the width of the phasel in the y dimension at the CGH plane is given by a length $\eta_{\mathrm{c}}$, then the transmission function for a single phasel can be written as follows. Note 
that the variable $\xi$ is used to represent the $\mathrm{x}$ dimension at the $\mathrm{CGH}$ plane and the variable $\eta$ is used to represent the y dimension at the CGH plane. Different variables will be used to express the $\mathrm{x}$ and $\mathrm{y}$ dimensions at the FPA plane:

$$
\begin{aligned}
& t_{\text {Single.Phasel }}(\xi, \eta)=e^{\frac{j 2 \pi\left(n_{\lambda}-1\right) d}{\lambda}} \operatorname{rect}\left(\frac{\xi}{\xi_{c}}\right) \operatorname{rect}\left(\frac{\eta}{\eta_{c}}\right) \\
& t_{\text {Single.Phasel }}(\xi, \eta)=e^{j \Phi_{\lambda}} \operatorname{rect}\left(\frac{\xi}{\xi_{c}}\right) \operatorname{rect}\left(\frac{\eta}{\eta_{c}}\right)
\end{aligned}
$$

One period of the CGH grating, referred to as the "unit cell" of the grating, is composed of a discrete number of phasels, as shown in figure 4.3. This same unit cell pattern is repeated again and again across the entire surface of the CGH. In general, the unit cell will be composed of either an $8 \times 8$ pattern of phase elements, $10 \times 10$ phasels, $12 \times 12$ phasels, or possibly higher number of phasels. The transmission of a single unit cell of the CGH can be expressed by combining the transmission of each phasel of the unit cell at its particular location, as shown in the following mathematical expression:

$$
\begin{aligned}
& t_{\text {CGH.UnitCell }}(\xi, \eta)= \\
& e^{j \Phi_{00}} \operatorname{rect}\left(\frac{\xi}{\xi_{c}}\right) \operatorname{rect}\left(\frac{\eta}{\eta_{c}}\right)+e^{j \Phi_{01}} \operatorname{rect}\left(\frac{\xi}{\xi_{c}}\right) \operatorname{rect}\left(\frac{\eta-\eta_{c}}{\eta_{c}}\right)+e^{j \Phi_{02}} \operatorname{rect}\left(\frac{\xi}{\xi_{c}}\right) \operatorname{rect}\left(\frac{\eta-2 \eta_{c}}{\eta_{c}}\right)+\ldots+ \\
& e^{j \Phi_{10}} \operatorname{rect}\left(\frac{\xi_{-}-\xi_{c}}{\xi_{c}}\right) \operatorname{rect}\left(\frac{\eta}{\eta_{c}}\right)+e^{j \Phi_{11}} \operatorname{rect}\left(\frac{\xi_{-} \xi_{c}}{\xi_{c}}\right) \operatorname{rect}\left(\frac{\eta-\eta_{c}}{\eta_{c}}\right)+e^{j \Phi_{12}} \operatorname{rect}\left(\frac{\xi_{-} \xi_{c}}{\xi_{c}}\right) \operatorname{rect}\left(\frac{\eta-2 \eta_{c}}{\eta_{c}}\right)+\ldots+ \\
& e^{j \Phi_{20}} \operatorname{rect}\left(\frac{\xi-2 \xi_{c}}{\xi_{c}}\right) \operatorname{rect}\left(\frac{\eta}{\eta_{c}}\right)+\ldots
\end{aligned}
$$
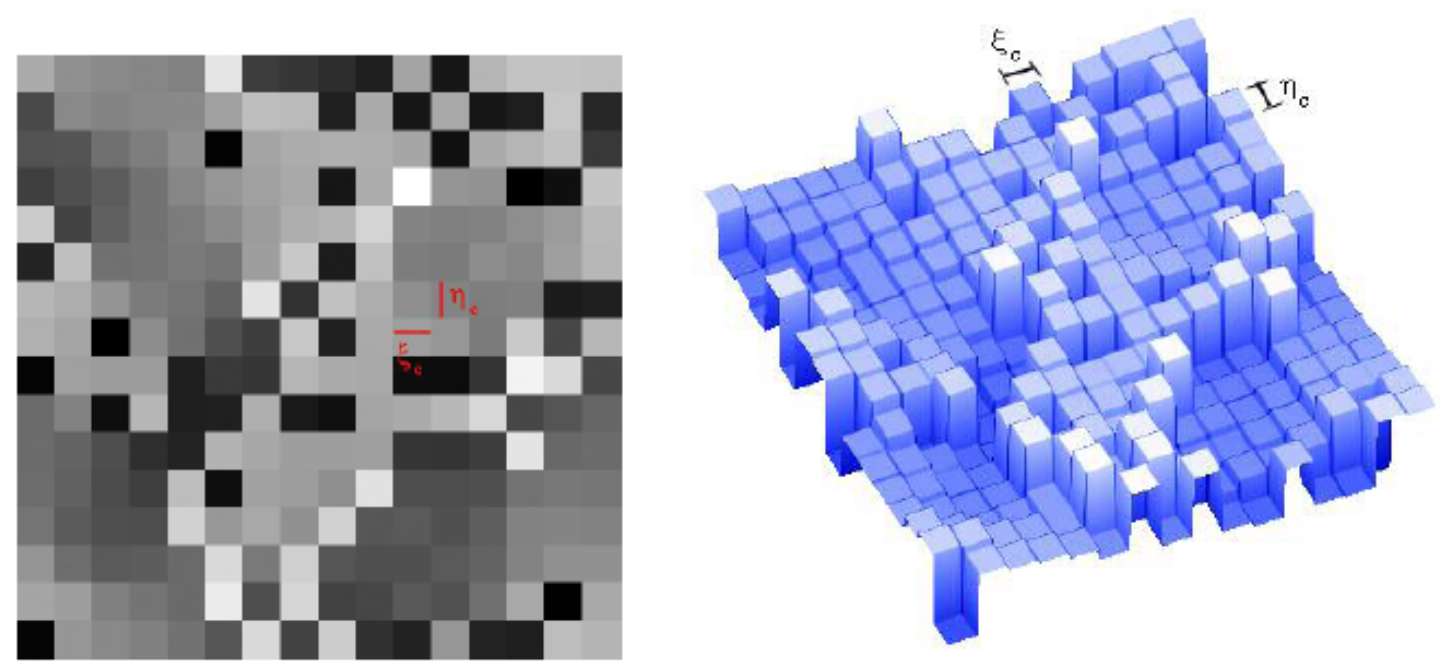

Figure 4.3: (left) Grayscale representation of a $16 \times 16$ phasel CGH unit cell, q=16. (right) 3dimensional plot of the same CGH unit cell illustrating the different thicknesses of each phasel.

This expression for the unit cell can be rewritten as: 


$$
t_{\text {CGH.UnitCell }}(\xi, \eta)=\sum_{m, n=0}^{q-1} e^{j \Phi m n} \delta\left(\xi-m \xi_{c}\right) \delta\left(\eta-n \eta_{c}\right) * *\left[\operatorname{rect}\left(\frac{\xi}{\xi_{c}}\right) \operatorname{rect}\left(\frac{\eta}{\eta_{c}}\right)\right]
$$

Where $\mathrm{q}$ is the number of phasels in each direction of a single unit cell (for example $\mathrm{q}=10$ for a $10 \times 10$ phasel unit cell), $\xi_{\mathrm{c}}$ and $\eta_{\mathrm{c}}$ are the real width and height of each CGH phasel $\left(\xi_{\mathrm{c}}=\eta_{\mathrm{c}}\right.$ for square phasels), and ** represents a two dimensional convolution.

This unit cell, which describes a single period of the 2D grating, is repeated across the entire surface of the CGH. The transmission of the entire CGH can now be expressed as:

$$
\begin{aligned}
& t_{\text {Entire.CGH }}(\xi, \eta)=\{\text { UnitCell }\}^{* *} \sum_{k, l=-\infty}^{\infty} \delta\left(\xi-k\left(q \xi_{c}\right)\right) \delta\left(\eta-l\left(q \eta_{c}\right)\right) \\
& t_{\text {Entire.CGH }}(\xi, \eta)=\left\{\sum_{m, n=0}^{q-1} e^{j \Phi m n} \delta\left(\xi-m \xi_{c}\right) \delta\left(\eta-n \eta_{c}\right) * * \operatorname{rect}\left(\frac{\xi}{\xi_{c}}\right) \operatorname{rect}\left(\frac{\eta}{\eta_{c}}\right)\right\} \\
& * * \sum_{k, l=-\infty}^{\infty} \delta\left(\xi-k\left(q \xi_{c}\right)\right) \delta\left(\eta-l\left(q \eta_{c}\right)\right)
\end{aligned}
$$

Where the first term is the same mathematical description of the unit cell as in equation (4.4) and the second term is the unit cell replication term. Since the unit cell is composed of q phase elements in each direction, the terms $\left(\mathrm{q} \xi_{\mathrm{c}}\right)$ and $\left(\mathrm{q} \eta_{\mathrm{c}}\right)$ are the physical lengths and widths of the unit cell, or stated in another way, $\left(\mathrm{q} \xi_{\mathrm{c}}\right)$ and $\left(\mathrm{q} \eta_{\mathrm{c}}\right)$ are the grating periods in the horizontal and vertical dimensions. The replication term consists of a grid of delta functions with integer grating period spacings in each dimension. The two dimensional convolution of the unit cell with the grid of dirac delta functions will replicate the unit cell at each of the dirac delta positions, effectively replicating the phase pattern over all the periods of the $2 \mathrm{D}$ grating.

From the previous chapter covering the optical layout of the CTIS, we know that the CGH in a CTIS system is located in the collimated section of the system between the collimating lens and reimaging lens. We can describe the field at the focal plane of the system, which is located at the rear focal plane of the reimaging lens, and how it is related to the transmission of the CGH with the following expression: ${ }^{11}$

$$
\mathrm{E}_{F P A}(x, y)=\frac{A}{j \lambda f} \times \iint_{-\infty}^{\infty} t_{\text {Entire.CGH }}(\xi, \eta) \exp \left[-j \frac{2 \pi}{\lambda f}(\xi x+\eta \mathrm{y})\right] d \xi d \eta
$$

This equation assumes that a monochromatic planewave of amplitude $\mathrm{A}$ is incident onto the $\mathrm{CGH}$, that the reimaging lens is a converging lens of focal length $\mathrm{f}$, and that the CGH is placed at the front focal point of the reimaging lens. If the $\mathrm{CGH}$ was placed at some other location in front of the reimaging lens, for example placed directly in front of the lens, the equation describing the field at the FPA would be the same shown above, but would include a quadratic phase factor preceding the integral. Irregardless of its specific position, we see that the field at the focal plane 
is proportional to the Fourier transform of the $\mathrm{CGH}$ transmittance function evaluated at $\mathrm{x}^{\prime}=\mathrm{x} / \lambda \mathrm{f}$ and $y^{\prime}=y / \lambda f$.

$$
\left.\mathrm{E}_{F P A}(x, y) \propto \mathfrak{I}\left\{t_{\text {Entire.CGH}}(\xi, \eta)\right\}\right|_{x^{\prime}=\frac{x}{\lambda \mathrm{f}}, y^{\prime}=\frac{y}{\lambda \mathrm{f}}}
$$

For the particular case of having the $\mathrm{CGH}$ at the front focal point, the field at the focal plane is described by the following equation.

$$
\mathrm{E}_{F P A}(x, y)=\frac{A}{j \lambda f} \times\left.\mathfrak{I}\left\{t_{\text {Entire.CGH}}(\xi, \eta)\right\}\right|_{x^{\prime}=\frac{x}{\lambda \mathrm{f}}, \mathrm{y}^{\prime}=\frac{y}{\lambda \mathrm{f}}}
$$

It is important to note that the camera will ultimately measure the irradiance at the focal plane. This example is shown to try and simplify the equations, but if the CGH was placed at a different location in the collimated space, the quadratic phase factor term would be of no consequence to the irradiance.

Since the transmission of the $\mathrm{CGH}$ is given as:

$$
\begin{gathered}
t_{\text {Entire.CGH }}(\xi, \eta)=\sum_{m, n=0}^{q-1} e^{j \Phi m n} \delta\left(\xi-m \xi_{c}\right) \delta\left(\eta-n \eta_{c}\right) * * \\
\operatorname{rect}\left(\frac{\xi}{\xi_{c}}\right) \operatorname{rect}\left(\frac{\eta}{\eta_{c}}\right) * * \\
\sum_{k, l=-\infty}^{\infty} \delta\left(\xi-k\left(q \xi_{c}\right)\right) \delta\left(\eta-l\left(q \eta_{c}\right)\right)
\end{gathered}
$$

We can calculate that the Fourier transform of the CGH transmittance function evaluated at $\mathrm{x}^{\prime}=$ $\mathrm{x} / \lambda \mathrm{f}$ and $\mathrm{y}^{\prime}=\mathrm{y} / \lambda \mathrm{f}$ is:

$$
\begin{aligned}
\left.\mathfrak{J}\left\{t_{\text {Entire.CGH}}(\xi, \eta)\right\}\right|_{x^{\prime}=\frac{x}{\lambda f}, y^{\prime}=\frac{y}{\lambda f}}= & \sum_{m, n=0}^{q-1} e^{j \Phi m n} e^{-j 2 \pi m \xi_{c} \frac{x}{\lambda f}} e^{-j 2 \pi n \eta_{c} \frac{y}{\lambda f}} \times \\
& \left|\xi_{c}\right|\left|\eta_{c}\right| \operatorname{sinc}\left(\frac{\xi_{c} x}{\lambda f}\right) \operatorname{sinc}\left(\frac{\eta_{c} y}{\lambda f}\right) \times \\
& \sum_{k, l=-\infty}^{\infty} \delta\left(x-\frac{k \lambda f}{q \xi}\right) \delta\left(y-\frac{l \lambda f}{q \eta_{c}}\right)
\end{aligned}
$$

(For more detail on this mathematical derivation see Appendix A.)

The resulting field at the focal plane is: 


$$
\begin{gathered}
\mathrm{E}_{F P A}(x, y)=\frac{A}{j \lambda f} \times \sum_{m, n=0}^{q-1} e^{j \Phi m n} e^{-j 2 \pi m \xi_{c} \frac{x}{\lambda f}} e^{-j 2 \pi n \eta_{c} \frac{y}{\lambda f}} \times \\
\left|\xi_{c}\right|\left|\eta_{c}\right| \operatorname{sinc}\left(\frac{\xi_{c} x}{\lambda f}\right) \operatorname{sinc}\left(\frac{\eta_{c} y}{\lambda f}\right) \times \sum_{k, l=-\infty}^{\infty} \delta\left(x-\frac{k \lambda f}{q \xi_{c}}\right) \delta\left(y-\frac{l \lambda f}{q \eta_{c}}\right) \\
\text { where } \operatorname{sinc}(\mathrm{x})=\frac{\sin (\pi x)}{(\pi x)}
\end{gathered}
$$

From this equation, we know that the field is zero for all points except for the $\mathrm{x}$ and $\mathrm{y}$ locations where the delta functions are nonzero. The coordinates that have nonzero fields are where $\mathrm{x}=$ $\mathrm{k} \lambda \mathrm{f} /\left(\mathrm{q} \xi_{\mathrm{c}}\right)$ and $\mathrm{y}=1 \lambda \mathrm{f} /\left(\mathrm{q} \eta_{\mathrm{c}}\right)$, which directly corresponds location of the diffraction orders given in the previous chapter which were calculated using the grating equation and small angle approximation.

We can express the field at these nonzero locations $x_{k}=k \lambda f /\left(q \xi_{c}\right)$ and $y_{1}=1 \lambda f /\left(q \eta_{c}\right)$ with the following equation.

$$
\begin{aligned}
& \mathrm{E}_{F P A}\left(x_{k}, y_{l}\right)=\frac{A\left|\xi_{c}\right|\left|\eta_{c}\right|}{j \lambda f} \times \sum_{m, n=0}^{q-1} e^{j \Phi m n} e^{\frac{-j 2 \pi m k}{q}} e^{\frac{-j 2 \pi n l}{q}} \times \operatorname{sinc}\left(\frac{k}{q}\right) \operatorname{sinc}\left(\frac{l}{q}\right) \\
& k=-\infty, \infty \text { and } l=-\infty, \infty
\end{aligned}
$$

Looking at the last two terms, we see that the field at the diffraction order locations is proportional to:

$$
\begin{gathered}
\mathrm{E}_{F P A}\left(x_{k}, y_{l}\right) \propto \sum_{m, n=0}^{q-1} e^{j \Phi m n} e^{\frac{-j 2 \pi m k}{q}} e^{\frac{-j 2 \pi n l}{q}} \times \operatorname{sinc}\left(\frac{k}{q}\right) \operatorname{sinc}\left(\frac{l}{q}\right) \\
k=-\infty, \infty \text { and } l=-\infty, \infty
\end{gathered}
$$

This first term is very similar to the two dimensional Discrete Fourier Transform (DFT) used in many computing software programs, shown in the equation below, but with $u_{m n}=e^{j \Phi m n}$. (Note that $\mathrm{U}_{\mathrm{kl}}$ and $\mathrm{u}_{\mathrm{mn}}$ are complex numbers.)

$$
U_{k l}=\frac{1}{M N} \sum_{m=0}^{M-1} \sum_{n=0}^{N-1} u_{m n} e^{-j 2 \pi\left(\frac{m k}{M}+\frac{n l}{N}\right)}
$$

So the field at the diffraction orders is proportional to a component of the two dimensional Discrete Fourier Transform of the unit cell phase values times a scaling sinc factor. 


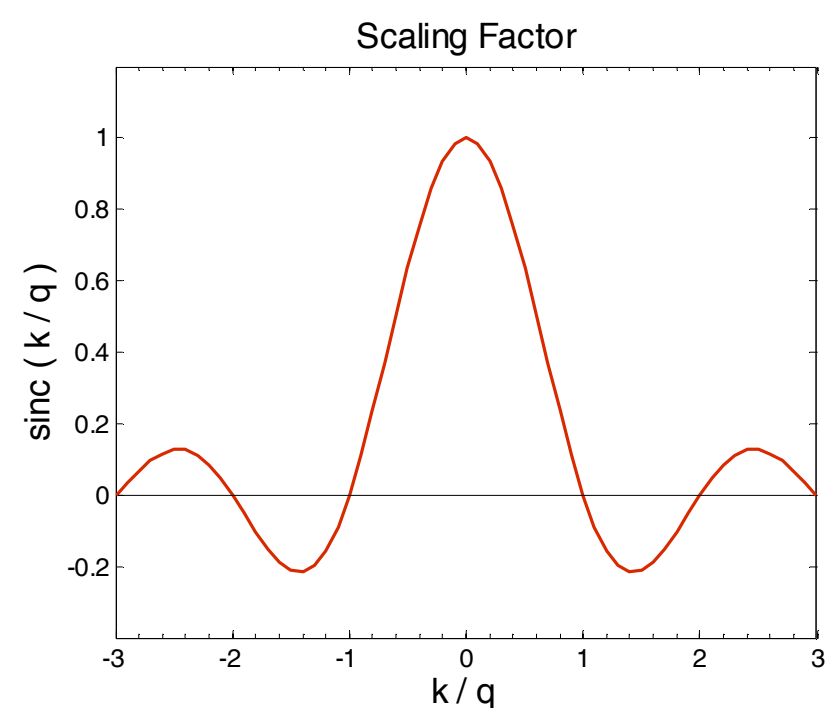

Figure 4.4: Plot of the sinc function that reduces the field at each diffraction order location

Note that excluding the scaling term, the far field pattern will repeat every q orders, since $\mathrm{U}_{(\mathrm{k}+\mathrm{q})(1+\mathrm{q})}=\mathrm{U}_{(\mathrm{k})(\mathrm{l})}$. Also note that the sinc scaling factor is solely due to the finite size/ finite height and width of each phase element. When taking into account the scaling term, shown in figure 4.4 , there will be $2 \mathrm{q}$ orders in the first lobe of the scaling sinc factor. For diffraction orders whose order numbers correspond to an integer multiple of q, excluding the zero order, the field at these orders will be zero, since they are located at the zeros of the scaling factor.

When relating this to a physical CTIS system, recall that the CGH will be designed to primarily send light into a specific pattern, such as a $3 \times 3$ array of diffraction orders. These desired orders are just a subsection of the $\mathrm{q} \times \mathrm{q}$ array of diffraction orders associated with a unit cell composed of $\mathrm{q} \times \mathrm{q}$ phasels. Without the scaling sinc term, that $\mathrm{q} \times \mathrm{q}$ pattern would repeat again and again every q orders. For example, with a $3 \times 3$ CTIS using a grating period with 8 phasels in each direction, without the scaling sinc factor, that $3 \times 3$ diffraction pattern would repeat every 8 orders. When taking into account the scaling term, the zero order of the diffraction pattern, which gets repeated every q orders, will now correspond to the center of the sinc function in the first lobe of the scaling term as well as the zeros of the sinc function, which are the locations of each repetition of the zero order. This means that if the CGH is designed to only send light into orders close to the zero order, these nearby orders will have the least scaling in the center lobe of the sinc function, but will simultaneously have the most suppression for the outer lobes of the sinc function. The orders in the center lobe will be the ones that are recorded by the focal plane, while the outer lobes corresponding to higher diffraction orders are not recorded and will fall off the focal plane.

This provides some insight when designing a CTIS CGH. In fact it will put a limit on the minimum number of phasels you can use to design the $\mathrm{CGH}$. If you want to create a $7 \mathrm{x} 7$ diffraction order pattern, there will need to be at least $7 \times 7$ phasels in the unit cell of the $\mathrm{CGH}$. When more phasels are used, the lower the field amplitude scaling effect will be on your desired orders near the zero order. In other words by increasing the value of q, the number of phasels, to be ever larger, the central lobe of the scaling factor in figure 4.4 will become wider. Keeping the 
desired orders near the central peak or expanding the central lobe allows the attenuating scaling factor for these orders to be closer to one resulting in greater efficiency for the CGH.

This analysis can be used to predict the diffraction efficiencies of the CGH as well as determining the appropriate alignment position of the CGH in the CTIS optical system. Going back to the example of the $\mathrm{CGH}$ placed at the front focal point of the reimaging lens. The field at the focal plane will be given by:

$$
\mathrm{E}_{F P A}\left(x_{k}, y_{l}\right)=\frac{A\left|\xi_{c}\right|\left|\eta_{c}\right| q^{2}}{j \lambda f} \times U_{k l} \times \operatorname{sinc}\left(\frac{k}{q}\right) \operatorname{sinc}\left(\frac{l}{q}\right)
$$

The focal plane will measure the irradiance of the light at the focal plane. Using this equation the irradiance at each of the diffraction orders will be given as:

$$
\mathrm{I}_{F P A}\left(x_{k}, y_{l}\right)=\frac{A^{2}\left|\xi_{c}\right|^{2}\left|\eta_{c}\right|^{2} q^{4}}{\lambda^{2} f^{2}} \times\left|U_{k l}\right|^{2} \times \operatorname{sinc}^{2}\left(\frac{k}{q}\right) \operatorname{sinc}^{2}\left(\frac{l}{q}\right)
$$

In regards to the appropriate location of the $\mathrm{CGH}$ in the collimated space of the system, placing the $\mathrm{CGH}$ at the front focal point of the reimaging lens allowed for a simplified analysis of the field at the focal plane, without having to keep track of the quadratic phase factor. This placement allowed for a simplified analysis, but could cause problems when constructing a physical CTIS system. In practice, it is best to put the CGH immediately in front of the reimaging lens. The simplified analysis did not consider the real finite extent of the reimaging lens. When the finite aperture is included there can be some vignetting of the input CGH. To avoid the issue of vignetting it is best to place the $\mathrm{CGH}$ directly against the reimaging lens and have the aperture of the lens be larger than the aperture of the CGH. The resulting irradiance will still be the same as given in the above equation. ${ }^{12}$

So the irradiance of light at a particular diffraction order is related to the square of the discrete Fourier transform (DFT) times the square of the scaling sinc functions. This mathematical description allows us to calculate the diffraction efficiencies as a function of wavelength for each

of the diffraction orders of the grating.

\subsection{Basic CGH Design Theory - How to Design the CGH Phase Pattern to Produce a Particular Irradiance Distribution}

In the design of the $\mathrm{CGH}$, we want to be able to select any diffraction pattern we chose, such as a $3 \times 3$ CTIS pattern or $5 \times 5$ CTIS pattern, and have high diffraction efficiencies for the selected orders at the selected wavelengths, while keeping the efficiency of the outer orders as close to zero as possible. We can now use this method of calculating the far field irradiance as a function of wavelength as a way to evaluate the performance of a particular unit cell phase distribution. The challenge in the design of the $\mathrm{CGH}$ is to be able use the results of this evaluation to find ways to make changes to the phase distribution that will improve the performance of the CGH and send more light where we want it to be in the CTIS image. 
There are several algorithms that can be used to specify the CGH phase distribution to produce a particular far field irradiance pattern. One of the traditional methods is the Gerchberg Saxton algorithm. ${ }^{13}$ The following is an outline for the steps of the Gerchberg Saxton algorithm.

\subsection{Gerchberg-Saxton Algorithm}

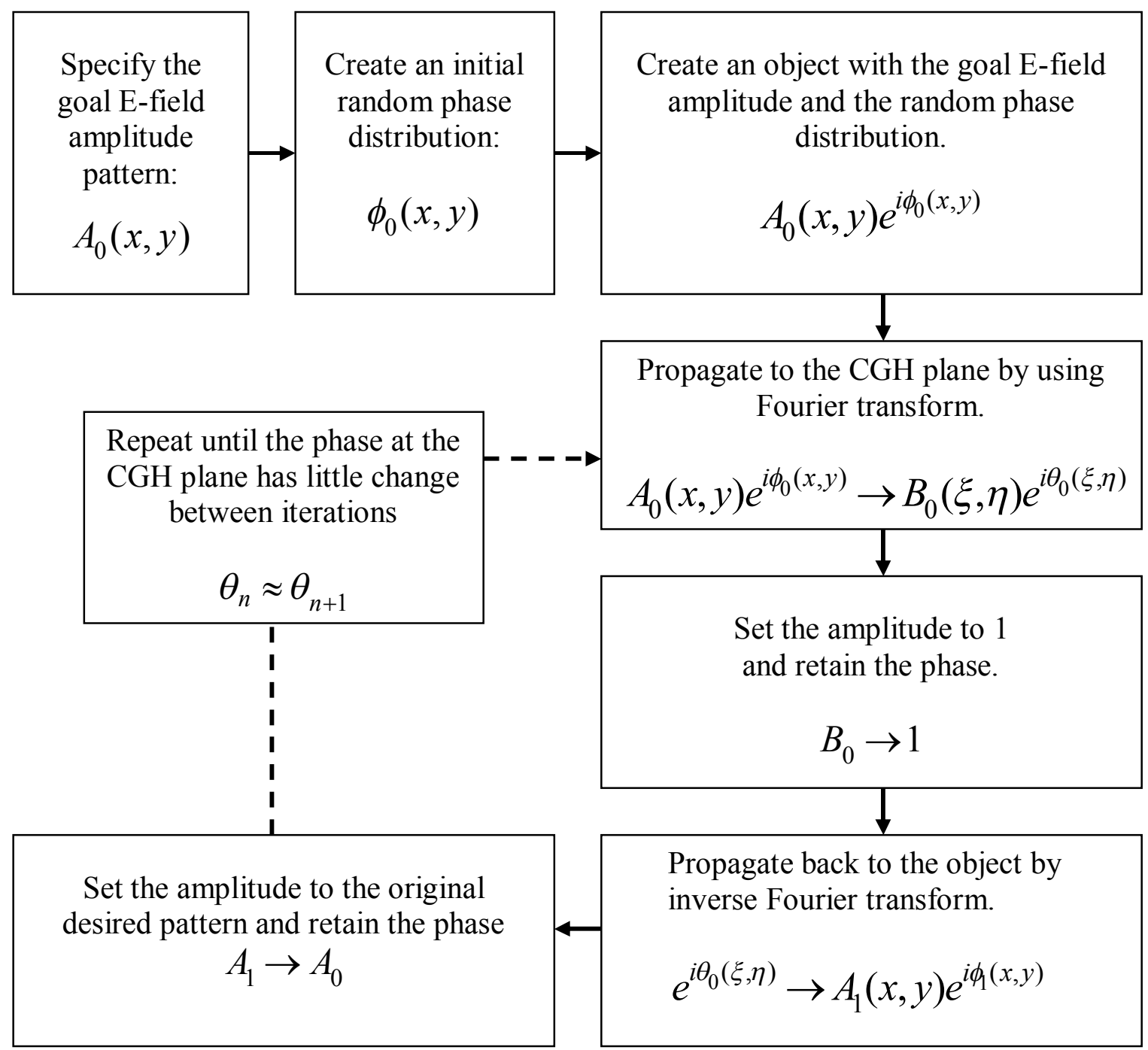

Unfortunately, this algorithm is only able to design a CGH phase profile that produces the desired irradiance pattern at a single wavelength. When the phase profile produced using this algorithm is converted into a surface profile of various thicknesses in an optical material, only a single wavelength will see the prescribed phase profile that will produce the correct irradiance pattern. There is no feedback mechanism that will allow the change in the phase distribution to incorporate the effects at different wavelengths. When other wavelengths pass through the CGH, they will experience a phase of: 


$$
\Phi_{\text {given wavelength, } \lambda_{2}}=\Phi_{\text {design wavelength, } \lambda_{1}}\left(\frac{\lambda_{1}}{\lambda_{2}}\right)\left(\frac{n_{\lambda 1}-1}{n_{\lambda 2}-1}\right)
$$

And will produce an irradiance pattern different than the original design. The CTIS imaging spectrometer must function over a wide range of wavelengths. If there is too much variation in the irradiance pattern as a function of wavelength for the gratings/CGH, a different approach must be used to design the phase profile.

\subsection{Polychromatic CGH Design Algorithm}

A CGH algorithm developed by Curtis Volin ${ }^{14}$, which uses a linear algebra based approach incorporating Singular Value Decomposition (SVD), is able to overcome this issue and design $\mathrm{CGH}$ phase profiles capable of operating at a number of wavelengths simultaneously. The approach relies on having a linear relationship between the CGH phases, $\Phi$, and the resulting electric field amplitudes, E. Unfortunately, there is no direct linear relationship between these values, but there is, however, a proven linear relationship between changes in the phase of the CGH and changes in the amplitudes in the diffraction plane. By mapping the resulting change in E-field at all the specified wavelengths for a small perturbation or change in phase of each phase element, a sensitivity matrix, S, can be formed relating the changes in CGH phase to changes in electric field amplitude at any wavelength.

The basic operation of the algorithm is to start with an initial randomly selected phase distribution. This random phase distribution will have an associated electric field distribution for all of the selected wavelengths. By calculating the difference between the electric field design the CGH is trying to achieve and the electric field produced by the current phase distribution, and by using the linear relationship between changes in the electric field and changes in the CGH phase, an update term for the initial phase selection can be produced. This update term will change the current phase profile to values that better produce the goal E-field at all of the specified wavelengths. This process can be repeated until the solution for the CGH phase converges or the produced E-field meets the performance requirements of the CGH.

The method of obtaining the phase update is as follows. The sensitivity matrix, S, relates changes in CGH phases to changes in the electric field amplitude.

$$
(\Delta E)=S(\Delta \Phi)
$$

There will be a difference between the produced electric field and the desired electric field that is related to some change in phase of the $\mathrm{CGH}$

$$
\left(E_{\text {design goal }}-E_{\text {current }}\right)=S\left(\Phi_{\text {goal }}-\Phi_{\text {current }}\right)
$$

By using the sensitivity matrix information and something like the inverse of the sensitivity matrix, we can solve for the phase update term that will improve the performance of the CGH. 
$\Phi_{\text {goal }}=\Phi_{\text {current }}+\Delta \Phi_{\text {update }}$

$\left(E_{\text {design goal }}-E_{\text {current }}\right)=S\left(\Delta \Phi_{\text {update }}\right)$

$S^{-1}\left(E_{\text {design goal }}-E_{\text {current }}\right)=S^{-1} S\left(\Delta \Phi_{\text {update }}\right)$

$S^{-1}\left(E_{\text {design goal }}-E_{\text {current }}\right)=\Delta \Phi_{\text {update }}$

However since the sensitivity matrix, $\mathrm{S}$, is a rectangular matrix, instead of using the inverse of $\mathrm{S}$ (which does not exist for rectangular matrices) the pseudoinverse of $\mathrm{S}, \mathrm{S}+$, is used instead. This pseudoinverse of $\mathrm{S}$ is calculated using the singular value decomposition (SVD) of the sensitivity matrix. This approach adds many more controls to the design of the CGH. The sensitivity matrix can incorporate evaluations of the electric field at multiple wavelengths and even different goal patterns at different wavelengths. If certain diffraction orders are considered more important than others weighting factors can be incorporated for specific orders, even at specific wavelengths. The weighting factors are especially useful in designs where many of the orders are intended to have no light, since these blank orders often outnumber the more significant orders where light is sent to and would otherwise provide more contributions in the sensitivity matrix. An outline of the steps of the polychromatic design algorithm is presented below. 


\subsection{Creating a CGH Operating at a Multiple Wavelengths that Includes Weighting Factors For Each of the Diffraction Orders}

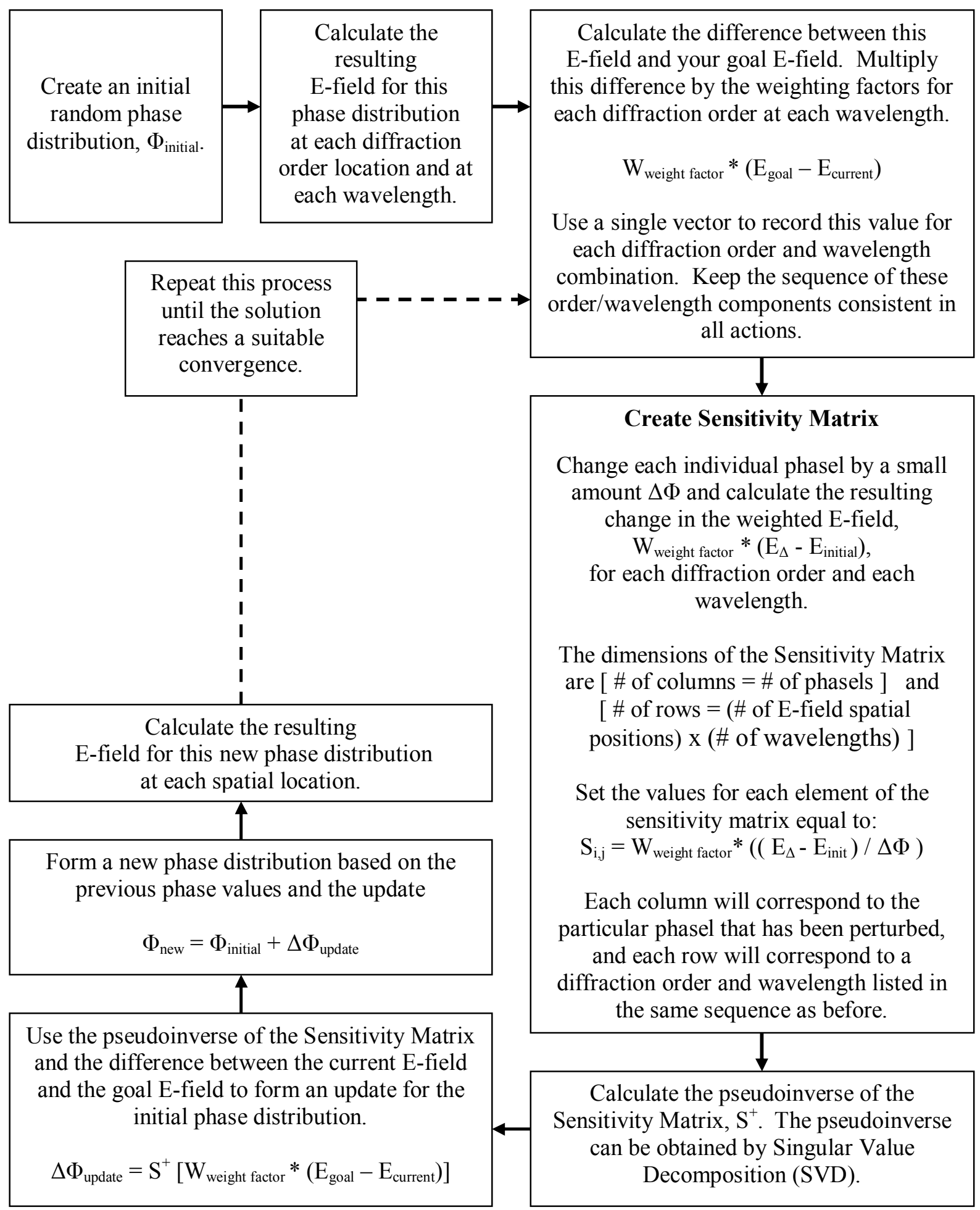




\subsection{Effect of Material Dispersion in Produced CGH}

Note that this polychromatic CGH design algorithm is creating a phase profile for the CGH and that this phase distribution is specified for a particular center wavelength within the spectral region. The algorithm is able to obtain polychromatic performance with this single phase pattern by incorporating the difference in electric field at multiple wavelengths into the sensitivity matrix. When evaluating the electric field distribution for different wavelengths in the algorithm, the phase profile associated with each wavelength is determined with the following relationship to the center design wavelength.

$$
\Phi_{\text {given wavelength, } \lambda_{2}}=\Phi_{\text {design wavelength, } \lambda_{1}}\left(\frac{\lambda_{1}}{\lambda_{2}}\right)
$$

When the CTIS CGH is manufactured, the phase profile values for the center wavelength must be converted into a physical thickness of optical material that will produce those same phase values at the center wavelength. To be explicit, the CGH algorithm designs to phase values and not material thicknesses. The algorithm does not take into account any change in index of refraction as a function on wavelength in the material. For materials with dispersion, the actual phase associated with these other wavelengths is given as:

$$
\Phi_{\text {given wavelength, } \lambda_{2}}=\Phi_{\text {design wavelength, } \lambda_{1}}\left(\frac{\lambda_{1}}{\lambda_{2}}\right)\left(\frac{n_{\lambda 1}-1}{n_{\lambda 2}-1}\right)
$$

Luckily, there is still an easy way to use the polychromatic CGH design algorithm and take into account any dispersion of the material. First consider the case of a material where the index of refraction is constant as a function of wavelength, but when creating the CGH an incorrect value for the index of refraction was used when determining the material thicknesses. The center wavelength will no longer experience the correct phase distribution and will produce a different irradiance pattern with different diffraction efficiencies in each of the orders. There will however, be another wavelength that is slightly offset from the design center wavelength that will experience the correct phase as the design center wavelength and will produce the same diffraction efficiencies as the original design. That wavelength can be calculated using the following equation.

$$
\lambda_{\text {actual }}=\left(\frac{n_{\text {actual value }}-1}{n_{\text {incorrect value }}-1}\right) \lambda_{\text {center design }}
$$

If the value for the index of refraction used to determine the material thickness was larger than the actual index of refraction, the wavelength that produces the same diffraction efficiencies as the original design is shifted to a slightly lower wavelength. If the value for the index was too low, the new wavelength that will have the desired diffraction efficiencies will be slightly higher than the design wavelength. For the manufactured CGH, there will be a new set of wavelengths, slightly offset from the original design, that will experience the design phase delays. So the net result of using the incorrect index of refraction is that the plot of diffraction efficiency as a function of wavelength will be slightly shifted left or right in wavelength. 
For materials with dispersion, the index of refraction of the material at the center wavelength should be used to determine the physical thicknesses of the CGH. Since the index of refraction changes as a function of wavelength, other wavelengths will not experience the same phase distributions as originally intended in the design and will have different diffraction efficiencies than originally designed. A different wavelength, which is slightly shifted from the original, will experience the designed phase distribution and will have the desired diffraction efficiencies. Since the index of refraction is changing as a function of wavelength, the shift for the corresponding wavelength that experiences the desired phases will also change as a function of wavelength. The result is that the plot of diffraction efficiencies as a function of wavelength will be stretched or contracted in different wavelength regions depending upon how the index of the material changes from the center wavelength index value in those regions.

The designer can easily account for these changes by using some prior knowledge of the dispersion of the material that will be used to manufacture the CGH. All the designer needs to do is to scale the design wavelengths used in the CGH algorithm to the equivalent values depending upon the dispersion of the material. This will result in the algorithm creating a phase profile where the correct diffraction efficiencies are to be produced at the correct wavelengths.

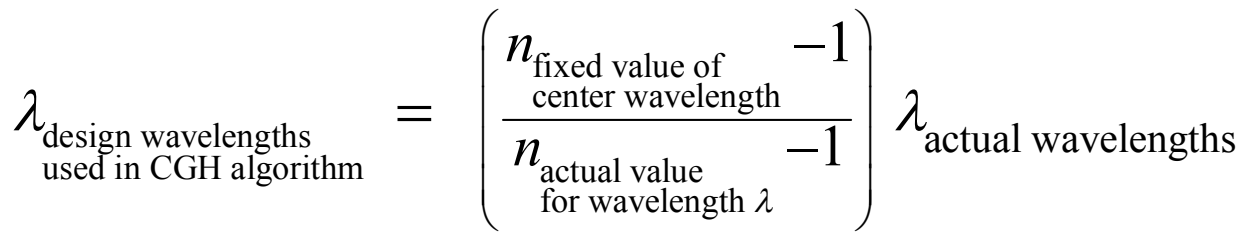

The final step in the CGH design is to determine the appropriate goal irradiance pattern for the CTIS imaging spectrometer system, the CGH algorithm will design the phase profile to produce. This problem is approached in two ways: for a given CTIS system with a specified diffraction pattern, what are the optimum diffraction efficiencies for each of the diffraction orders and which CTIS diffraction pattern is the best for the system? For the case of a given diffraction pattern the relative diffraction efficiencies for each diffraction order must be selected to avoid signal problems on the recording focal plane array.

\subsection{Selecting Relative Diffraction Order Efficiencies to Limit Issues of SNR}

The polychromatic CGH design algorithm allows the designer to select which diffraction orders light will be sent to as well as the relative diffraction efficiency for each order. While the selection of orders will determine the specific multiplexing of the spatial and spectral data in the recorded image, it is really the selection of the relative diffraction efficiencies for each order that will have the biggest effect on how that multiplexed data is measured. The optical engineer faces a challenge of selecting appropriate relative efficiencies such that the recorded CTIS images are useful in producing spectral reconstructions. If too much light is sent into one place in the CTIS image, the detector will saturate at that location. If there is too little light at a location, the output for that pixel will be dominated by noise. When this occurs, some of the 
spatial/spectral information from the scene is lost, decreasing the quality of the reconstructed data. The efficiency of each order in the CGH must be carefully designed to try and avoid these saturation and low signal problems.

The designer must answer, for a given CTIS diffraction pattern, what are the relative diffraction efficiencies of each order that best avoid saturation and low signal pixels? Another way to rephrase this question might be to ask what are the relative diffraction efficiencies of each order that produce a uniform SNR for all of the lit pixels on the focal plane? Unfortunately there is no general answer that works for all cases, because the specific efficiency values will depend upon the object viewed by the system. For a CTIS with a fixed CGH, the designer must first make some assumptions about the types of objects that will be viewed before selecting efficiencies.

Consider the case of imaging a purely monochromatic object with the CTIS. Since the object is composed of just a single wavelength, the image of that object will be replicated at one particular position in each of the diffraction orders. In order to produce a uniform SNR for the entire image, the diffraction efficiencies for all of the orders must all be the same. If the efficiency for one of the orders was higher than the others, then the lit pixels in that order would become saturated before any of the other pixels in the image.

Now consider the case of viewing a spectrally broadband object, while still keeping the relative efficiency of all of the orders the same. In the zero diffraction order of the image, all of the spectral signal for a particular spatial location of the object is added together for a single pixel's output. In the outer diffraction orders, that same amount of signal will be divided up over a range of pixels due to the dispersion in the order. Clearly the pixels in the zero order will have a much higher SNR and will be more likely to be saturated than those in the outer orders. The different spectral properties of the object will require different relative diffraction efficiencies for the orders.

For broadband objects the designer needs to decrease the relative efficiency of the zero order to avoid saturation. And to avoid saturation in the outer orders, the designer could weight the relative efficiencies for each of the orders based on the amount of dispersion in the order. This is a reasonable approach for broadband objects. Orders with more dispersion will have more light sent into that order to try and account for the added number of pixels the signal is distributed over. However, there are some drawbacks to this approach. One of the major drawbacks is that the zero order, which has no dispersion, would be completely blank. Even within a given diffraction order, for a broadband object with some spatial extent, there will be some pixels in the order that will have a higher signal than others. Recall that each pixel on the focal plane can receive light from points along a diagonal line through the datacube. Some pixels within a diffraction order will be able to receive light from more points in the datacube than others, and can result in higher SNRs. So, the goal of achieving uniform SNR for all of the lit pixels is still not met.

It turns out the only way to achieve uniform SNR on all of the lit pixels for any object viewed with the system is to have zero signal in each pixel, which is useless for a CTIS. The CGH can NOT be designed to produce uniform SNR for all pixels for all objects. We have just illustrated how the efficiency weightings would need to be different for different objects, and how even 
within a given order certain pixels will have more multiplexing than others and can have a higher signal.

For systems that will only be viewing certain types of objects, the relative efficiencies should be tailored to the properties of those objects. Objects with primarily monochromatic features will have more uniform efficiencies. For objects with multiple strong emission lines, perhaps the dispersion of the system could also be increased to reduce the overlap of the monochromatic images in the orders. Objects with primarily broadband features will want to send more light into orders that have more dispersion.

If the designer has no prior knowledge about the properties of the objects the system will view, consider the following general approach. Select the relative efficiencies such that the light is evenly distributed based on the number of pixels that could possibly receive light by the given diffraction order. Note that this is not the same as ensuring every lit pixel will have the same SNR, but it is a general approach to try and limit the effects of low signal pixels and saturation and provides a compromise between the two extreme cases.

Using this method, the selection of the efficiencies will be uniquely tailored to each CTIS system, but makes no assumptions about the object properties. The approach takes into account both the size of the replicated monochromatic images and the amount of dispersion in the orders based on the overall CTIS design and the selected wavelength range. If there is minimal dispersion over the wavelength range and a large zero order image, the possible multiplexing will be limited, and the resulting relative diffraction efficiencies will be closer to being uniform, as in the monochromatic/single band case. If the system is designed with a large amount of dispersion compared to the zero order size, this approach will still provide a useful zero order image and while also sending more light into the longer diffraction orders.

Determining the relative efficiencies for a given CTIS using this method is also quite easy. There is a simple mathematical algorithm, called the shoelace method or shoelace algorithm, that can quickly calculate the area of any simple 2D polygon using only the $\mathrm{x}, \mathrm{y}$ coordinates of the vertices/corners of the polygon (a description of the method given in Appendix B). These coordinates for the CTIS can be calculated using the equations to find the location and size of the orders, presented in chapter 3 . The resulting relative diffraction efficiency values for the CTIS would be equal to the area of pixels a given diffraction order could possibly send light to divided by the area of pixels that the entire CTIS could possibly send light to. Even though the actual diffraction efficiencies of the resulting CGH will be different (i.e. the CGH will not be $100 \%$ light efficient) their relative values created by the CGH algorithm should be close to being the same values calculated by the ratio of pixel areas. By using these diffraction efficiency values in the CGH design algorithms, the phase pattern for the CGH will be selected in such a way, as to try and limit low signal and saturation issues for the recorded image.

\subsection{Selecting a CTIS diffraction pattern for a system}

Selecting a diffraction pattern for a CTIS is perhaps one of the trickiest parts in the design of a CTIS system because the analysis and comparison of these various patterns can be quite complex and computationally tasking. In the early history of CTIS, the diffraction patterns always moved 
toward incorporating more diffraction orders in the recorded image. However, recent work by Nathan Hagen has illustrated that the addition of more orders does not necessarily improve the performance of the system ${ }^{15}$. Some ongoing evaluations using computer simulations to model the effects of various designs has also helped dispel some of the perceived limitations on what types of objects can and can not be reconstructed by the CTIS, especially in regards to the "missing cone" in the Fourier representation of the datacube, but more work will need to be done in the future to provide to provide more definitive answers as to which pattern performs better under various conditions.

When selecting the diffraction pattern for a CTIS system there are a number of factors that can help influence and guide the selection process. Some of these factors include:

- The required spatial and spectral resolution for the system

- The resolution of the focal plane array used in the system

- The types of objects being viewed with the system

- Restrictions on the time required for reconstructing data

- and the amount of error you can tolerate in the reconstructed data

When using a fixed size focal plane array, the designer must ultimately try and balance the tradeoffs in spatial and spectral sampling for various diffraction patterns to meet the spectrometer's resolution requirements for the reconstructed data. The spatial resolution of the system is often given by the number of pixels sampling the zero order image on the focal plane, while the spectral resolution of the system is related to the number of pixels sampling the spectral dispersion in the longest diffraction order. By changing the size and location of the zero order as well as the locations, dispersion, and number of the outer diffraction orders on the recorded image, the designer controls the sampling of the multiplexed spatial and spectral data, which will affect how the data for a given scene is recorded and ultimately reconstructed.

For example, when comparing a $3 \times 3$ diffraction pattern with a $7 \times 7$ diffraction pattern on the same size focal plane array, as illustrated in figure 4.5 , it is clear that the $3 \times 3$ pattern will have significantly more spatial resolution due to the larger zero order (in the example CTIS there are about 80 times more spatial samples in the zero order for the $3 \times 3$ design), however there is less dispersion and fewer spectral samples per spatial location in the outer diffraction orders of the $3 \times 3$ pattern compared to the $7 \times 7$ pattern (the $7 \times 7$ pattern will record about $30 \%$ more spectral samples in the outermost diffraction orders in the example systems due to the higher grating dispersion). If there are strict requirements on spatial and spectral resolution for the imaging spectrometer system, the designer must select an appropriate pattern to try and meet those requirements. For example, if having a higher spectral resolution is a strict requirement and the spatial resolution of the system isn't as important it may be better to select a diffraction pattern with higher diffraction orders and more dispersion, however if having a high spatial resolution is a strong requirement of the system, the designer may be limited to using a lower order design. Of course all of these selections must take into account the resolution of the focal plane array used to record the image, as it will determine the sampling rate for a given diffraction pattern fitting on the FPA, and the resolution/quality of the reconstructed data for a given system. 


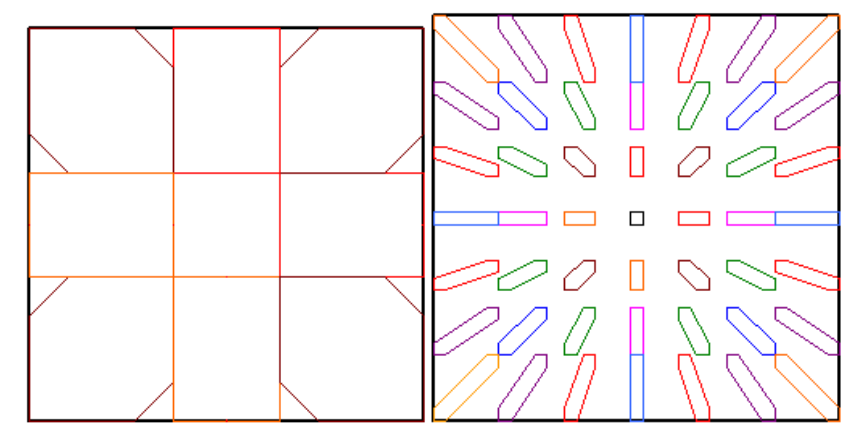

Figure 4.5: Comparison of a 3x3 CTIS diffraction pattern (left) and a 7x7 pattern (right) for a given CTIS system. Both systems use the same focal plane and the same spectral bandwidth. Even though the diffraction orders in the $3 \times 3$ are longer than the outermost orders of the $7 \times 7$ pattern recall that the total length of the order is equal to the size of the zero order plus the dispersion. So, even though the length of the orders is longer for the $3 \times 3$ pattern, there is more dispersion and thus more spectral samples for a given spatial location in the $7 \times 7$ pattern.

The analysis of determining the relative number of spatial and spectral samples in the image when comparing different diffraction patterns is fairly straight forward. However, things become more complicated when trying to relate these different samplings/different diffraction patterns to the reconstructed data for an object. When using the standard iterative reconstruction techniques it has been shown that the spectral resolution of a CTIS system is object dependent. ${ }^{16}$

Specifically, the spectral resolution of the system will be highest when viewing a point object. This is fairly intuitive, because in such cases the operator could effectively read the relative spectrum for the point object directly from any of the diffraction orders in the system. The same is true when using the standard iterative reconstruction techniques, the spectral resolution for a point object is limited by the Nyquist sampling of the spectrum in the diffraction orders, where the spectral resolution is equivalent to the spectral dispersion across 2 pixels in the outermost horizontal or vertical diffraction order. In the other extreme, the spectral resolution of a CTIS system will be its worst when viewing uniform broadband objects that fill the field of view of the CTIS. This is the case where the multiplexing of the recorded data in the system is maximized. The spectral resolution for such objects has been shown to be equivalent to the spectral dispersion across 14 pixels in the outermost horizontal or vertical diffraction orders for a $5 \times 5$ CTIS diffraction pattern. So the designer should choose an appropriate pattern based on the resolution requirements for the system, the sampling of the focal plane, as well as the types of objects intended to be viewed with the system.

When using the standard iterative reconstruction techniques, not only must the designer balance a tradeoff between spatial and spectral sampling for various patterns while trying to maintain a specified spectral resolution for various objects, there will also be slight tradeoffs in the quality of reconstructions for different diffraction patterns, different objects, different reconstruction techniques, different noise levels, and even different calibration techniques. It has been shown that for a given object, the amount of error in a datacube reconstruction can change for different diffraction patterns. ${ }^{17}$ See reference and figure 4.6. If at all possible the designer should try and model these effects in the time before designing and manufacturing the $\mathrm{CGH}$ to compare the performance for various diffraction patterns. Even though more simulation work will need to be done in the future to provide definitive answers as to which diffractions patterns will perform 
better when viewing various objects, having various noise levels, and using different reconstruction techniques, there are some general guidelines to comparing diffraction patterns.

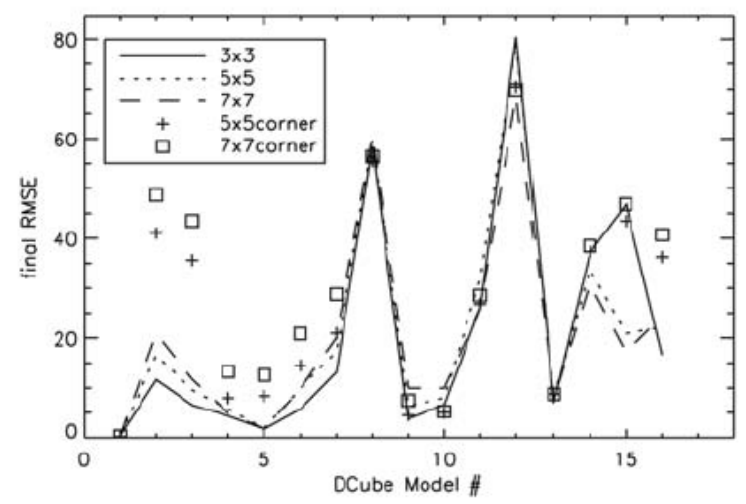

Figure 4.6: Plot from Nathan Hagen illustrating the various amounts of error (Root Mean Squared Error) for different diffraction patterns when viewing different object datacube models.

\subsection{Notes and a General Rule on Comparing Diffraction Patterns}

The general rule of thumb when comparing CTIS diffractions patterns, such as the two patterns in figure 4.7, is that systems with more unique diffraction orders will tend to have better spatial reconstructions, while systems with longer diffraction orders will tend to have better spectral reconstructions. However, not all diffraction orders are created equal. Some diffraction orders can contain redundant information of the multiplexing of the spatial and spectral data.
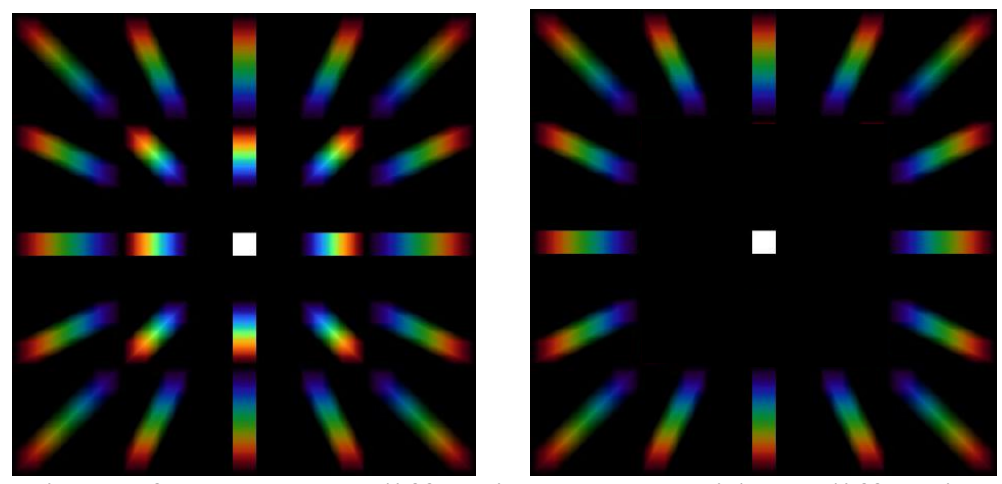

Figure 4.7: Comparison of a 5x5 CTIS diffraction pattern with 25 diffraction orders (left) and a $5 \times 5$ pattern consisting of only the 16 outermost diffraction orders (right)

For example in the $5 \times 5$ CTIS diffraction pattern, the inner ring of 8 diffraction orders all contain redundant information. Recall the linear nature of the grating's dispersion in each of the diffraction orders. All of the information in the plus one horizontal order, directly to the right of the zero order, is also contained in the plus two horizontal order since the light is linearly dispersed twice as much in the outer order than the inner order. The data in the inner, plus one, horizontal order could conceivably be recreated by binning pixel values in the plus two horizontal order. It is because these two orders share the same azimuthal angle relative to the zero order, that the information in the order with less dispersion is redundant. The "spectral smear" of the datacube data has different amounts but is in the same direction. 
Note that the same is not true when comparing the plus two horizontal diffraction order to the minus two horizontal diffraction order, since these two projections are at different azimuthal angles. The scene's multiplexed data in those orders is different since the spectral spread of the scene is in a different direction.

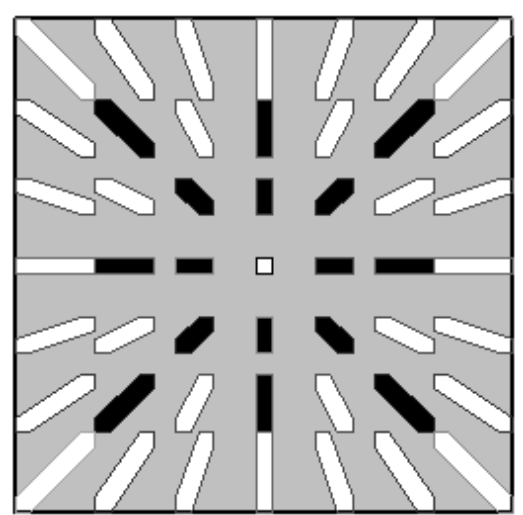

Figure 4.8: Illustration of the redundant diffraction orders (shown in black) for a 7x7 CTIS diffraction pattern.

Even though the inclusion of these redundant orders, highlighted in figure 4.8, may help reduce the effect of image noise in the reconstructed data, there are a number of reasons to design the CGH to try and eliminate these orders. The two main advantages are that the datacube reconstructions for the system can be calculated in a shorter amount of time and the relative diffraction efficiency of the outer orders could be increased, resulting in more light being able to be sent into each order increasing the SNR of the recording pixels. In general the amount of time needed to calculate a reconstructed datacube from an image depends upon the number of diffraction orders in the CTIS system, which will be discussed in a later chapter. By eliminating redundant orders, the calibration data for the system is simplified and large areas of pixels on the focal plane are no longer needed to determine the reconstructed data. The elimination of redundant orders allows the system to perform faster and reduces the noise in the recorded image. However, if you increase the size of your zero order to make more use of the focal plane, these additional lit pixels may negate some or all of the increase in reconstruction speed.

Another important note about the characterization of various CGH diffraction patterns is in regards to some of the metrics used to classify CGH design patterns. One such metric, called the "oversampling" coefficient, has been shown to be of limited use when comparing diffraction patterns to select for a system. The oversampling coefficient is calculated by determining the ratio of the number of possibly lit pixels on the focal plane of the CTIS image to the number of voxels in the datacube. The theory for this figure of merit was that this value would provide a sense for the number of "measurements" (pixel values) versus the number of "unknowns" (voxels) for a given system. The thinking was that systems with an oversampling coefficient greater than one would ensure that the system would be able produce quality reconstructions. Unfortunately this is an oversimplification of what is really occurring in a CTIS system and is not an entirely useful metric for comparing diffraction patterns for a number of reasons. As we have just illustrated, within a given CTIS image there may be some redundant information recorded in some of the lit pixels on the focal plane array. The inclusion of these pixels in the calculation of the metric would artificially inflate the oversampling coefficient for a system. The 
figure of merit also seems to imply that with merely enough lit pixels/measurements in the CTIS system, a reconstructed datacube could be determined by solving a system of linear equations. However, due the nature of the multiplexing in the system this completely deterministic reconstruction is not always possible. There are often a variety of "null vectors" for a given CTIS system, meaning that multiple different datacubes could generate the same recorded CTIS image and that a completely deterministic reconstruction is impossible. Even for systems, with no redundant orders, where the linear set of equations describing the multiplexing can be solved, the cutoff for these systems has no strict relation to the oversampling coefficient. For example some CTIS systems with an oversampling coefficient of 2.1 are able to determine a deterministic reconstruction while other systems with slightly larger spatial and spectral resolution that also have an oversampling coefficient of 2.1 can not. Perhaps the best evidence against the use of oversampling as a figure of merit is the fact that computer simulations for systems with a lower oversampling coefficient are still able to produce useful reconstruction data using the iterative reconstruction techniques.

As a final note on the diffraction patterns for the $\mathrm{CGH}$ grating and the comparison of various patterns, recall that the linear nature of the grating's dispersion in each of the diffraction orders will limit the number of spectral samples recorded by the focal plane based on the spectral bandwidth of the system. This is sometimes referred to as an "octave" problem, meaning that in order to avoid overlap between some of the diffraction orders the spectral bandwidth of the system must be limited to a specific range depending upon the order number of the diffraction orders being used. For example the $3 \times 3$ pattern, outer $5 \times 5$ pattern, and outer $7 \times 7$ pattern, have no restrictions on the highest wavelength of the spectral region relative to the lowest wavelength, since only a particular ring of orders are being used, with all the other orders being suppressed. However, even these patterns will still have a limitation on the size of the zero order and the number or recorded spectral samples in the orders based on the linear dispersion of the grating. This often results in a number of the pixels in the focal plane remaining blank that are not used to record the CTIS image. There are some new techniques for the design of the polychromatic CGH that incorporate something similar to a "grism" that are able to overcome this limitation and make better use of all of the pixels on the focal plane array. ${ }^{18}$ 


\section{CHAPTER 5: CTIS CALIBRATION AND RECONSTRUCTION OVERVIEW}

\subsection{CTIS System Information and Reconstruction}

In order to obtain reconstructed spatial and spectral data from a recorded CTIS image, some of the properties of the CTIS system must be known ahead of time. These properties of the system are measured through a calibration process, which records how each possible component used to describe an object datacube corresponds to certain pixel values on the recorded image. In the reconstruction of spatial and spectral data from a recorded CTIS image, the resulting information about the scene will be expressed as a linear combination of all of the different datacube components recorded in the calibration process. In effect, the reconstruction combines the various images corresponding to each possible component of the datacube, with different weights for each image/datacube component, such that the combined image is as similar as possible to the recorded image. It is the different weights used for each datacube component that provide the reconstructed spatial and spectral data for the scene.

This chapter will provide an overview of the reconstruction process, discuss the details of the CTIS system calibration, and provide the mathematical basis used for reconstruction and calibration. In addition, methods to simplify the calibration process and provide radiometrically correct reconstruction data will also be covered. Finally, specific details on the unique calibration procedure used for the LWIR CTIS will be presented.

\subsection{CTIS Reconstruction \& Calibration Overview}

Once the design and construction of the CTIS are complete, the system can finally be used to record images for various scenes. An example of an image recorded with the LWIR CTIS is shown in figure 5.1. In this example image, viewing a scene where the tip of a soldering iron is pointed at the exit slit of a monochromator, the general spectral properties of each object in the scene can be determined just by looking at the various diffraction orders. The soldering iron, which is the triangular shaped object in the zero order, must have broadband spectral properties in the LWIR because it produces a large spectral smear spanning nearly the entire length of each of the outer diffraction orders, while the exit slit of the monochromator, the rectangular object at the tip of the soldering iron, is clearly composed of monochromatic light since it only appears at one place in each of diffraction orders. 


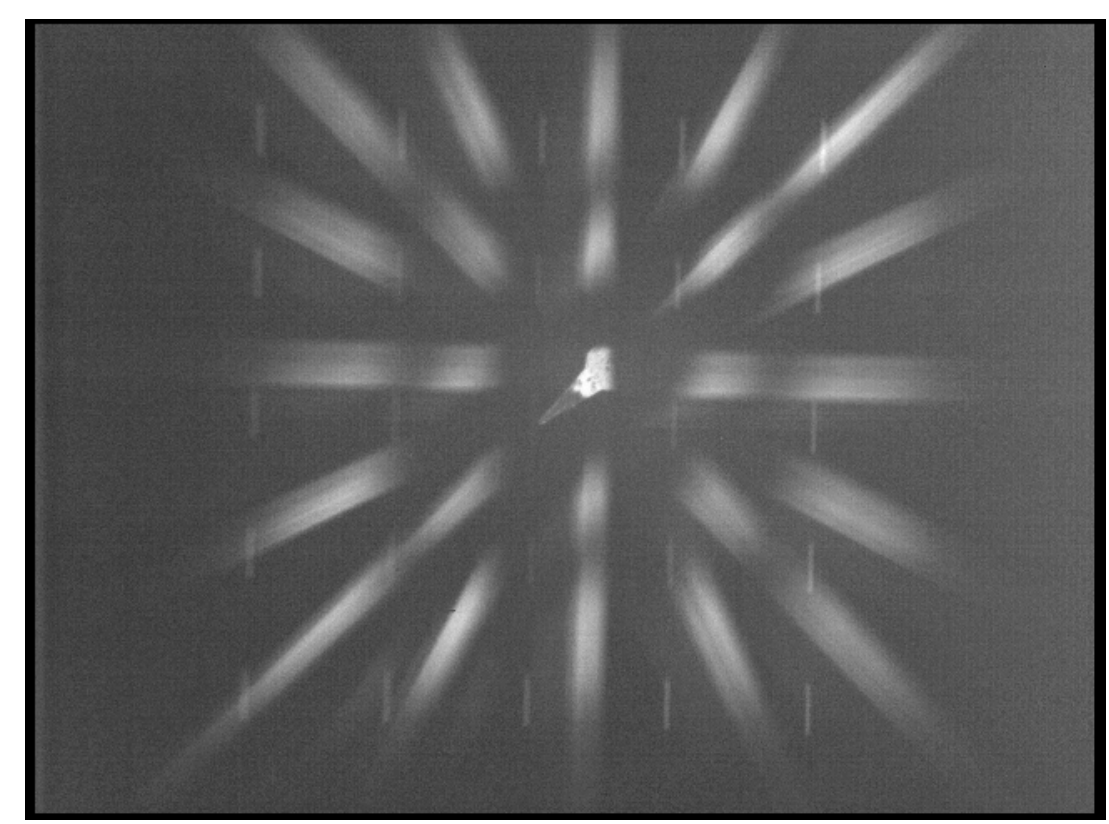

Figure 5.1: Recorded image from the LWIR CTIS viewing the tip of a soldering iron pointed at the exit slit of a monochromator.

The CTIS reconstruction process, which determines the irradiance values for all of the spatial and spectral positions in the datacube, aims to do a similar type of analysis, albeit in a much more mathematical and systematic way. By processing the raw data from the image in a particular manner, the information contained in the various diffraction orders can be analyzed in order to determine the spectral properties of all the points in the scene.

\subsection{Reconstruction Overview}

The standard imaging equation, $\mathrm{g}=\mathrm{Hf}$, provides both a general mathematical representation of how the recorded CTIS image is produced as well as the mathematical foundation of how the spatial and spectral properties of a scene can be reconstructed from the CTIS image data. In this equation, $\mathrm{g}$ represents the recorded CTIS image, $\mathrm{H}$ is a mathematical operator describing the optical system, and f represents the object datacube. In the equation $\mathrm{g}=\mathrm{Hf}$, we say that the optical system, $\mathrm{H}$, operates on the object, $\mathrm{f}$, to produce the recorded image, $\mathrm{g}$, as illustrated in figure 5.2 .
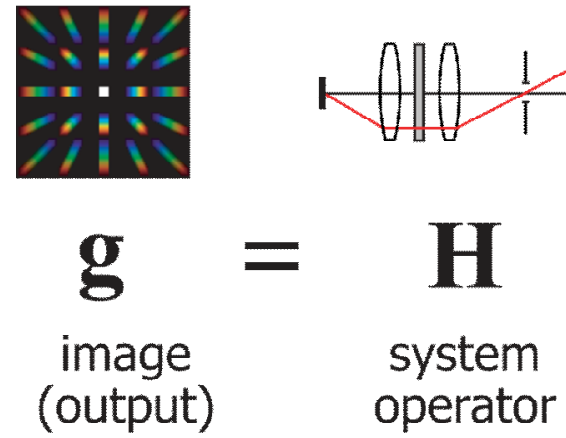
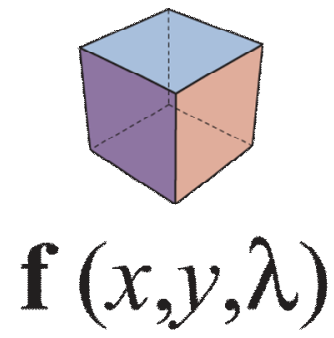

object (input)

Figure 5.2: Illustration of the imaging equation components in CTIS 
The reconstruction of data from a CTIS image will try to take this image formation process in reverse. By examining the recorded image, $\mathrm{g}$ (what we are given), and using the existing knowledge of the optical system, $\mathrm{H}$, the reconstruction process will try to determine the datacube, $\mathrm{f}$ (what we are trying to $f \mathrm{ind}$ ), that generated the original image.

Some of the specific mathematical reconstruction techniques used in CTIS are directly borrowed from medical tomography. Medical computed tomography (CT) systems reconstruct an estimate of a 3D object based on multiple projections of that object onto 2D planes. Since the diffraction orders of a CTIS image can be interpreted as projections of a 3D object datacube onto the $2 \mathrm{D}$ focal plane at different angles, as illustrated in figure 5.3, CTIS can use the same mathematical tomographic reconstruction procedures as medical CT systems to determine the three dimensional datacube information from the 2D information of the diffraction orders in the recorded image. These reconstructions, however, will still require some prior knowledge of the optical system (specifically a mathematical description of the system's H operator) to determine wavelength irradiances in the datacube. The specifics of the system $\mathrm{H}$ operator can be found by calibrating the system, as covered in the following section.

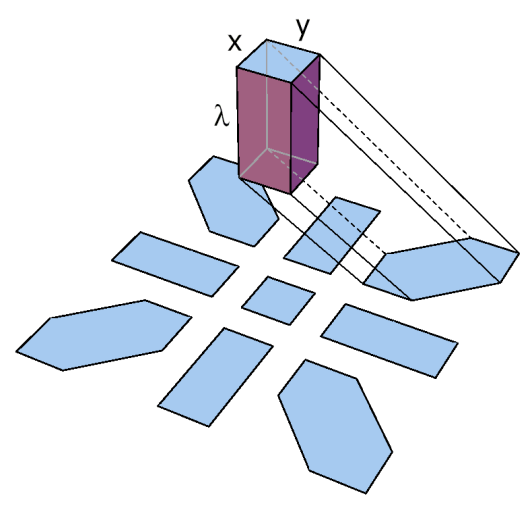

Figure 5.3: Diagram illustrating how each diffraction order of the CTIS can be interpreted as a projection of the 3D datacube onto the 2D focal plane array.

\subsection{Calibration Overview}

To illustrate the importance of $\mathrm{H}$, consider again the example CTIS image in figure 5.4. By looking at the diffraction orders, it was shown that the light emanating from the slit object was monochromatic. For imaging spectrometer systems, being able to identify specific spectral features in a scene is crucial in identifying and characterizing objects. However, with just the information presented in the image, even though it can be determined that the light leaving the slit is monochromatic, it is impossible to determine the specific wavelength of the monochromatic light emanating from the slit. The image alone does not contain enough information to make that determination. In order to identify the specific wavelength properties, we need more information about the system itself. By initially calibrating the CTIS system we can develop a mathematical representation of the system operator $\mathrm{H}$ and use that knowledge to determine specific features of the scene. 


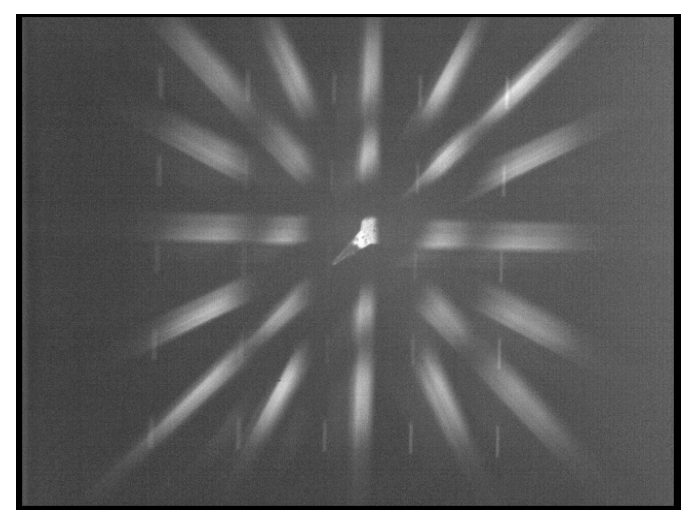

Figure 5.4: Recorded image from the LWIR CTIS viewing the tip of a soldering iron pointed at the exit slit of a monochromator.

In the example image with the soldering iron and monochromator slit, if it was already known how the different wavelengths of light at different spatial locations of the scene are mapped onto the focal plane to create the recorded images, the specific wavelength of light emanating from the slit could be determined by finding the corresponding wavelength of light, at the same spatial location as a point on the slit in the scene, that maps to the same locations of the replicated slit images in the outer diffraction orders. It is this prior knowledge that allows the specific wavelength of the monochromatic light to be determined. So in the calibration of the CTIS system, the mathematical description of the system operator $\mathrm{H}$ is created by measuring how all of the different components of the datacube are mapped onto the focal plane array by the optical system. By recording how each component of the datacube description corresponds to particular pixel values at specific locations of the recorded image, this calibration information/description of the system $\mathrm{H}$ operator will be used to reconstruct specific datacube values from the recorded images for any scene.

\subsection{CTIS Calibration Process}

The CTIS calibration information can be obtained by a variety of different ways depending on how the spatial and spectral properties of the scene are represented. In this dissertation, the spatial and spectral properties of the scene are represented as being a collection of discrete voxel irradiance values, with each voxel corresponding to a small spatial area and a given wavelength band in the scene. Other descriptions of the datacube may use a different basis to describe the properties of the scene, such as a Fourier basis, and will require different calibration techniques and different recorded calibration data. To complete the calibration of a CTIS and construct the system $\mathrm{H}$ operator, the user must record how each possible component of the description of the datacube maps to pixel values in the recorded image. In the case of using voxels to describe the datacube, a CTIS image will need to be recorded for each voxel used in the datacube description.

These CTIS images for each voxel are obtained by physically recording images of monochromatic point objects, and a separate CTIS image will need to be recorded for every monochromatic point/voxel in the datacube. This collection of images, referred to as calibration images, provide information on how all the different spatial and spectral components of the scene relate to pixel values on the recorded image. 
Each calibration image can be viewed as the "impulse response function" for a particular component of the datacube. The mathematical description of the system $\mathrm{H}$ operator is composed of the collection of the impulse response functions from all of the possible datacube components. Stated in another way, the mathematical description of the H operator is just a collection of calibration images for the system. During the reconstruction process, the estimate of the object datacube that created the CTIS image is given by a linear combination of these calibration images. So with the voxel description, the reconstructed estimate of the datacube is a collection of differently weighted monochromatic points at different spatial locations in the scene. If the reconstruction of the datacube is similar to the datacube of the actual scene used to record the image, then the weighted sum of all of the calibration images should produce an image that closely matches the recorded CTIS image.

\subsection{Recording Single Voxel Calibration Images}

To physically record these single voxel calibration images, a monochromator and an optical fiber are often used to produce a monochromatic, spatially point like object that is then imaged by the CTIS. The fiber can either be imaged by the system's objective lens or the fiber can be placed directly at the field stop, as shown in figure 5.5. With the optical fiber held at a fixed location, the wavelength output of the monochromator is scanned, and a CTIS image is taken at each wavelength step.

Each calibration image corresponds to a particular voxel, and this collected set of data, with the fiber held at a fixed location, provide the calibration images for an entire column of the datacube. The complete description of the $\mathrm{H}$ operator for the system requires a calibration image at all of the possible datacube locations, so a similar set of calibration images must be taken at each defined spatial location of the scene. This exhaustive approach can be done experimentally, however, by making an assumption that the imaging of the optical system is spatially shift invariant, the same set of wavelength data from a single spatial location can be modified to generate the required images for all of the remaining spatial locations.

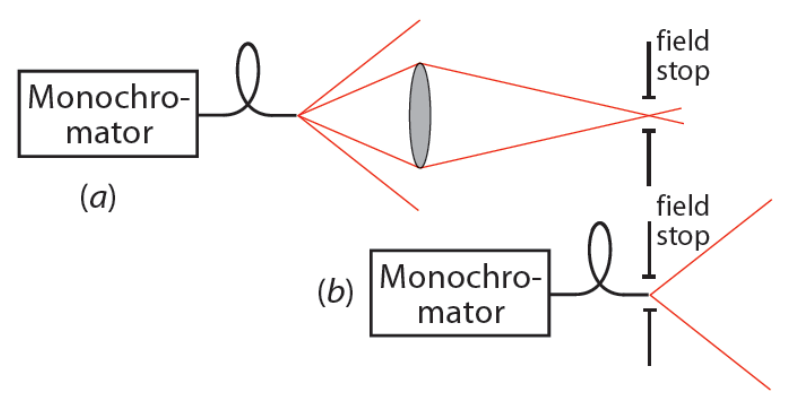

Figure 5.5: (a) The entire CTIS system, including the objective lens, images the monochromatic light output of an optical fiber to record a calibration image. (b) The objective lens from the CTIS is removed, and the optical fiber is placed directly at the field stop to record calibration images. 


\subsection{Simplified Calibration Approach and the Shift Invariance of CTIS}

For an entire optical system to be spatially shift invariant, all of the field dependent aberrations in the system must be balanced to zero, which by itself can be a reasonable assumption for many CTIS systems due to the limited diffraction angles of light exiting the CTIS disperser. However, in regards simplifying the calibration process of a CTIS system, the restrictions for shift invariance in the system are even looser. In a calibration image, each diffraction order will have its own associated point spread function. To use the argument of shift invariance to simplify CTIS calibration, the only requirement is that the corresponding point spread function (PSF) for each diffraction order at a particular wavelength be shift invariant over the small area of the diffraction order corresponding to the monochromatic image replication of the field stop at that wavelength. The PSF could vary as a function of wavelength and could vary from diffraction order to diffraction order, but the same wavelength calibration data from a single spatial location could still be used to generate all of the needed calibration images for the H operator. The system need only be shift invariant over the small area of the monochromatic image of the field stop in each diffraction order to allow calibration data for the entire datacube to be generated from a set of calibration images describing a single column in the datacube.

\subsection{How to create the system $\mathrm{H}$ operator}

For the work in this dissertation, the standard imaging equation, $\mathrm{g}=\mathrm{Hf}$, will be expressed as a matrix vector multiplication, using vectors to describe the recorded image and object datacube and a matrix description of the system $\mathrm{H}$ operator. The $\mathrm{g}$ vector describing the image will contain the recorded pixel values, while the datacube vector, $\mathrm{f}$, is composed of spectral irradiance values for each voxel. In the example imaging equation below, there are $\mathrm{M}$ pixels in the recorded image, $\mathrm{N}$ discrete voxels in the datacube vector, and the system $\mathrm{H}$ matrix is an $\mathrm{M}$ by $\mathrm{N}$ matrix.

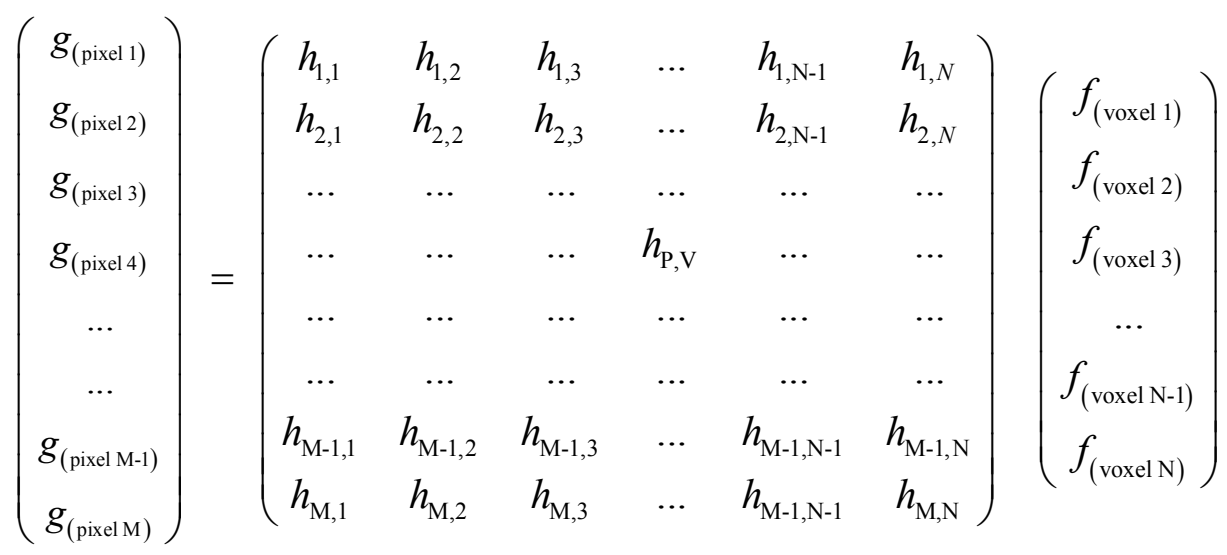

Each individual element of the $\mathrm{H}$ matrix will relate a single voxel to a single pixel. For example, the specific element of the $\mathrm{H}$ matrix, $h_{\mathrm{P}, \mathrm{V}}$, is the contribution to the output of pixel $\mathrm{P}$ (as a digital number or pixel signal value) for one unit of light in the voxel V. With this representation, it is clear that the total signal output for each pixel will be determined by the sum of all these contribution factors times the amount of light in their corresponding voxels. 


$$
g_{(\text {pixel 1) }}=\left(h_{1,1} \cdot f_{(\text {voxe 1 })}\right)+\left(h_{1,2} \cdot f_{(\text {voxe 2 })}\right)+\left(h_{1,3} \cdot f_{(\text {voxel 3) }}\right)+\ldots+\left(h_{1, N} \cdot f_{(\text {voxel N) }}\right)
$$

Another way to understand the components in the $\mathrm{H}$ matrix, and to be able to create the $\mathrm{H}$ matrix for a given system, is to consider the case where the system is viewing a scene that is only composed of a single lit voxel. In the vector representation of the datacube, the component for the arbitrarily chosen lit voxel, voxel $\mathrm{V}$, will be equal to one, while the irradiance for all of the other voxels will be zero, as shown in the following equation.

$$
\begin{aligned}
& \text { single lit voxel } \Rightarrow f_{\text {voxel V }}=1 \text {, all others }=0
\end{aligned}
$$

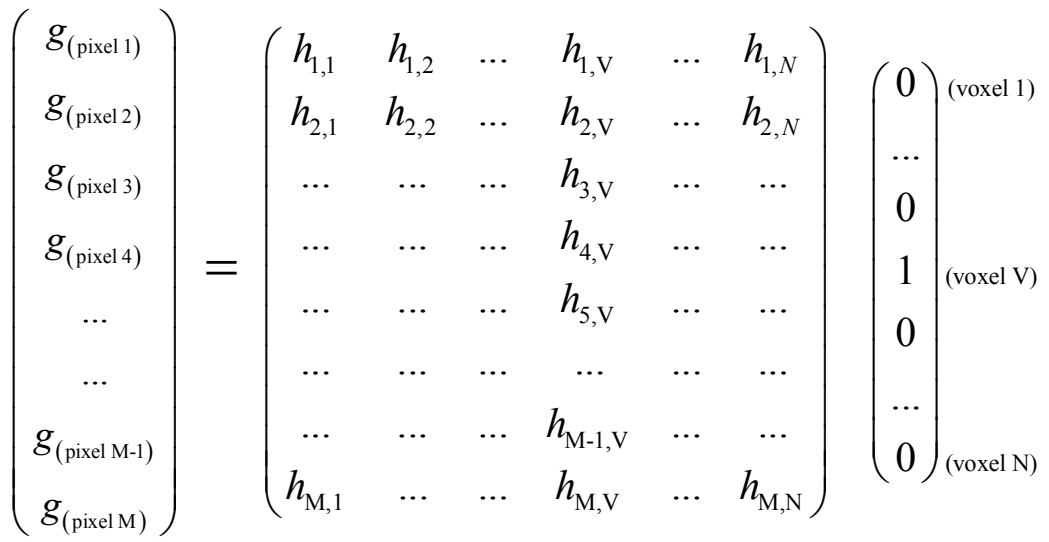

$$
\begin{aligned}
& \text { recorded image single voxel }
\end{aligned}
$$

By performing the matrix multiplication, it is found that the recorded image for this single voxel object is equal to a particular column of the $\mathrm{H}$ matrix. If a scene composed of a different voxel were viewed, the recorded image would equal a different column in the $\mathrm{H}$ matrix. In other words each column of the $\mathrm{H}$ matrix is composed of the pixel values for the calibration image associated with a particular voxel. In order to construct the $\mathrm{H}$ matrix for a system, a calibration image must be obtained for each possible voxel in the datacube, and the set of calibration images will form all the columns of the system $\mathrm{H}$ matrix.

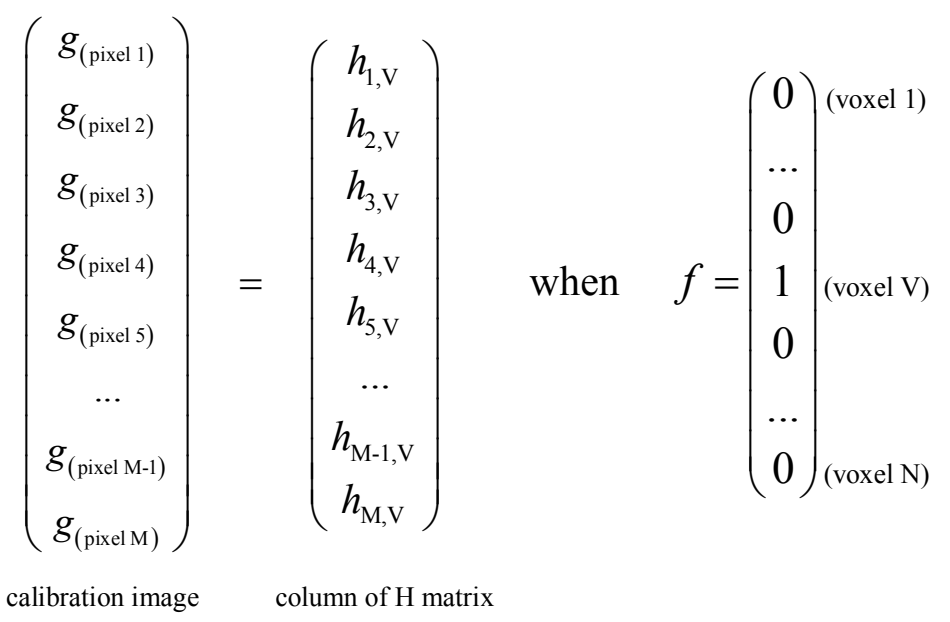




\subsection{Radiometric Calibration}

Note that with this method, the unit describing the amount of light in each voxel of the datacube is a relative value. Having a value of one for a particular component of the datacube vector means that the irradiance in that voxel is equal to the irradiance of the monochromatic point object that generated the corresponding calibration image for that voxel. So all of the irradiance values in the reconstructed datacube vector will just be values relative to the voxel irradiance that created each calibration image. For imaging spectrometer systems, this is often not sufficient. There is often a requirement to have radiometrically correct reconstructed data for the system. To obtain radiometrically correct datacube values, there needs to be a slight modification to the values composing the system $\mathrm{H}$ matrix.

Spectral irradiance has units of watts per meter squared per nanometer. Since the 2D spatial extent and spectral bandwidth of each voxel in the datacube description is the same, the irradiance value for each voxel is directly related to the radiant power, in watts, of each datacube voxel. Provided that the integration time and all the other camera settings, such as the camera's gain and offset, are fixed for all the images taken with the system (including both calibration images and images that will be reconstructed), the only modification needed is to normalize the pixel values in each calibration image by the collected radiant power of the monochromatic point source generating the image. This is physically done by using a calibrated power meter to measure the power of the light exiting the optical fiber, for each wavelength band, that is collected by the system. By dividing the pixel values in the calibration image by the recorded power that generated the image, the components of the $\mathrm{H}$ matrix will now have units of Digital Numbers per Watt. Reconstructions using this $\mathrm{H}$ matrix will produce estimates of the collected radiant power from each voxel in actual (non relative) radiometric units, watts.

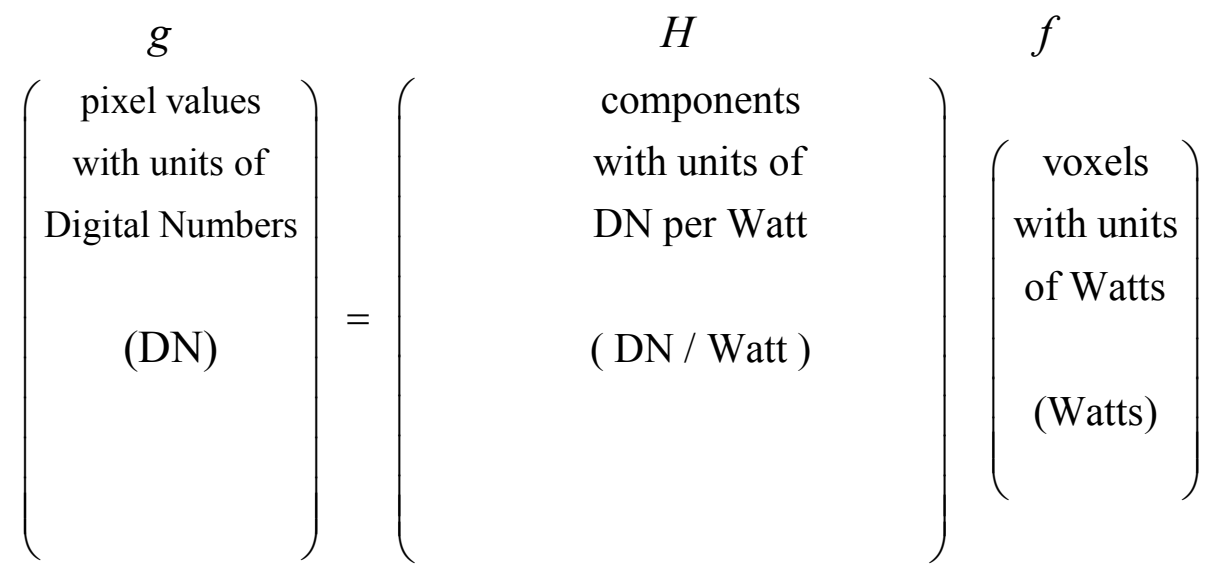

Since there can sometimes be a large difference between the signal collected in a calibration image and the collected signal for an actual scene, another modification to the system can be made to produce radiometrically calibrated datacube values that account for different integration times used in different images. Again, this is assuming that all of the other camera's settings are fixed and that the recorded signal is within the linear response region of the detector. The radiometrically correct $\mathrm{H}$ matrix for this case will be formed by dividing the pixel values of each calibration image by the integration time for the calibration image as well as the collected radiant power of the monochromatic point generating the calibration image. The recorded image, $g$, 
must also be normalized by its integration time. Reconstructions using the normalized image vector, irregardless of its integration time, and the modified $\mathrm{H}$ matrix will produce datacube voxel power estimates in units of watts.

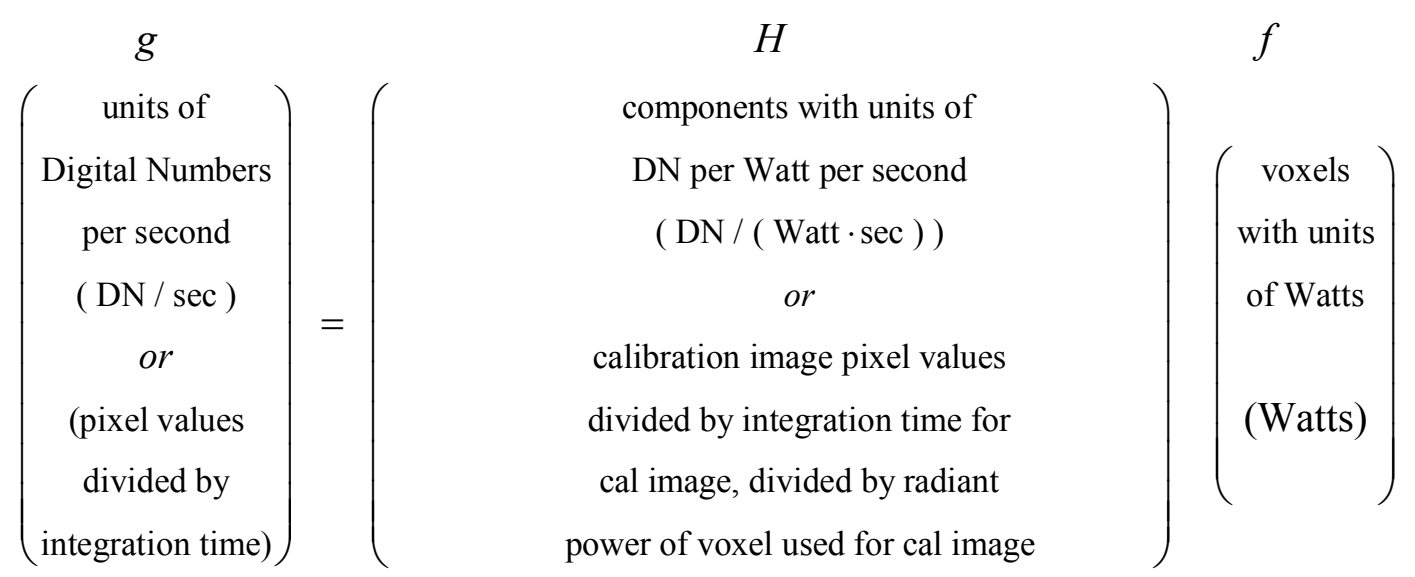

\subsection{Calibration images and sampling of reconstructed data}

The number of calibration images used in the system is arbitrarily defined by the user. This is important because the number of irradiance values produced in the reconstructed datacube estimate is equal to the number of system calibration images. The user is free to take as many or as few different calibration images as deemed appropriate, but there are some physical parameters of the system that will limit the usefulness of certain approaches.

This does NOT necessarily imply that the resolution of the CTIS system will be increased merely by using more calibration images in the optical system description, H. Even though the reconstructed data may have more data points, the resolvable resolution of the system may not have changed, since the resolution parameters are often related to physical properties of the system such as the physical size of the pixels and the amount of dispersion in a diffraction order, as well as the types of objects being viewed. ${ }^{19}$

A reasonable approach to determine the appropriate number of calibration images is to try and take calibration images corresponding to integer pixel steps on the focal plane. For example, a set of calibration images would be acquired at the spatial locations of the scene corresponding to the center of each pixel in the zero order. The separation of the wavelength bands in the datacube would also be selected such that the difference between two adjacent wavelength bands corresponds to a shift of one pixel, from the center of one pixel to the center of the adjacent pixel, in the diffraction order with the highest amount of dispersion.

The problem is that the actual spatial and spectral properties of the scene are given by continuous functions. This discrete representation, corresponding to integer pixel steps, can give a reasonable, but not perfect, description of the object. There will always be a particular spatial location or particular wavelength that maps to a position right at the edge of two pixels. The user can record calibration images for these intermediary datacube positions, but the signal on the focal plane array will be divided out and interpolated over multiple pixels. By acquiring 
calibration images at finer than integer pixel steps, the user can try and obtain sub pixel resolution, but this adds complexity to the reconstruction and $\mathrm{H}$ matrix, and may not always be beneficial since the resolvable resolution of most CTIS systems corresponds to steps somewhat larger than a single pixel.

\subsection{Challenges in the calibration of the LWIR CTIS system}

There are two factors that can limit the system's ability to record the monochromatic point images necessary for the calibration of a CTIS. These two factors are the loss of light when generating a monochromatic point object and the limited sensitivity of the detector focal plane array. At times, these factors can even make it impossible to record spatially and spectrally point like objects with a CTIS, in which case new approaches towards the calibration of the system must be taken.

The most straight forward ways to combat these issues are to use extremely sensitive detectors and increase the amount of light in the point objects. However, these may not always be feasible options. In the case of the LWIR CTIS, it was a requirement that the system use an uncooled detector. This requirement lead to the selection of a silicon microbolometer focal plane array, which is far less sensitive than other LWIR detectors, such as a cooled Mercury Cadmium Telluride ( $\mathrm{HgCdTe}$ ) FPA. So in many situations it may not always be possible to increase the sensitivity of the detector.

In regards to increasing the amount of light in the monochromatic point objects, there are often many places for improvement due to the many sources of loss in the system. Some of the parameters that affect the amount of light in the generation of a monochromatic point are: the radiance of the source, the limited collection of radiation from the source sent to the monochromator, the f number of light sent into the monochromator relative to the acceptance angle of the monochromator, the entrance slit size and the size of the source image at the entrance slit of the monochromator, the diffraction efficiency of the monochromator grating, the exit slit size, the coupling efficiency into the optical fiber, the numerical aperture of optical fiber's output, the position and size of the optical fiber, and the acceptance angle of the CTIS system. The net result of all these effects is that for a limited brightness source, only a very small fraction of that light is sent into the CTIS system when recording a calibration image, and that small amount of light may not be enough to overcome the noise levels in the detector due to the sensitivity of the detector.

The ideal radiation source would be a high radiance point emitter that has a directional output matching the acceptance angle of the monochromator. In the LWIR, the radiance of the source will often be related to the temperature and emissivity of the object and Planck's law. For the calibration of the LWIR CTIS the highest temperature source available, a 1000 degree Celsius blackbody source with a 1 inch aperture, was used as the input to the monochromator. Wein's Law states that the wavelength with the peak radiant emittance for this source is around 2.3 microns and that the radiant emittance will continually decrease at higher wavelengths. Again, there were many factors contributing to the loss of throughput when recording calibration images, but even when optimizing many of these parameters the system was unable to record 
calibration images at 12 micron wavelengths due to the source's blackbody falloff of emittance at higher wavelengths.

The solution to these issues was to use a "synthetic" approach to calibration. This synthetic approach uses a combination of physically recorded data and computer simulations to generate the calibration images used in the system $\mathrm{H}$ matrix. An example of such a synthetic calibration image is shown in figure 5.6.

Similar approaches have been used in previous visible CTIS systems, and the approach implemented in the LWIR system is an extension of this previous work. In the visible CTIS systems, the primary problem faced in the calibration images was the extremely narrow spectral bandwidth of each voxel. When trying to record calibration images the signal within that narrow bandwidth was too small to be detected by the system. The solution was to open up the slits of the monochromator, increasing the spectral bandwidth of the light entering the CTIS, and thus increasing collected signal. However the calibration images were no longer spectrally point like. Each diffraction order of the image consisted of a larger area of lit pixels. Since the spectral bandwidth of the output of the monochromator is related to the convolution of the entrance slit with the exit slit, so long as both slits are of the same size, and assuming that the emission of the source, the diffraction efficiencies of the gratings, and the responsivity of the detector have little change over narrow spectral bandwidth, the peak of the transmitted spectral bandwidth will be at the center wavelength. So to mitigate the affect that the recorded calibration images consisted of multiple wavelengths and large areas of lit pixels in each diffraction order, the centroid signal location was calculated in each diffraction order and associated with the center wavelength. The synthetic calibration image for the center wavelength is generated based upon this recorded image by estimating the point spread function in each order and applying that psf at the centroid location in the generated image. For the calibration of the LWIR CTIS, the same approach was taken, but for the wavelengths where the monochromatic point object could not be recorded an estimate of the point spread function of each order was made based on the specific wavelength and the psf of wavelengths that could be recorded in the system. The centroid location for the wavelengths was calculated based on the centroid locations of the recorded images and the linear spectral dispersion of the grating in each of the orders.

This approach can actually further simplify the calibration process of a CTIS system. Not only can you use shift invariance to generate many of the calibration images of the system. For a fixed spatial position, images at only a handful of wavelengths need to be recorded and the remaining wavelength calibration images can be generated. 


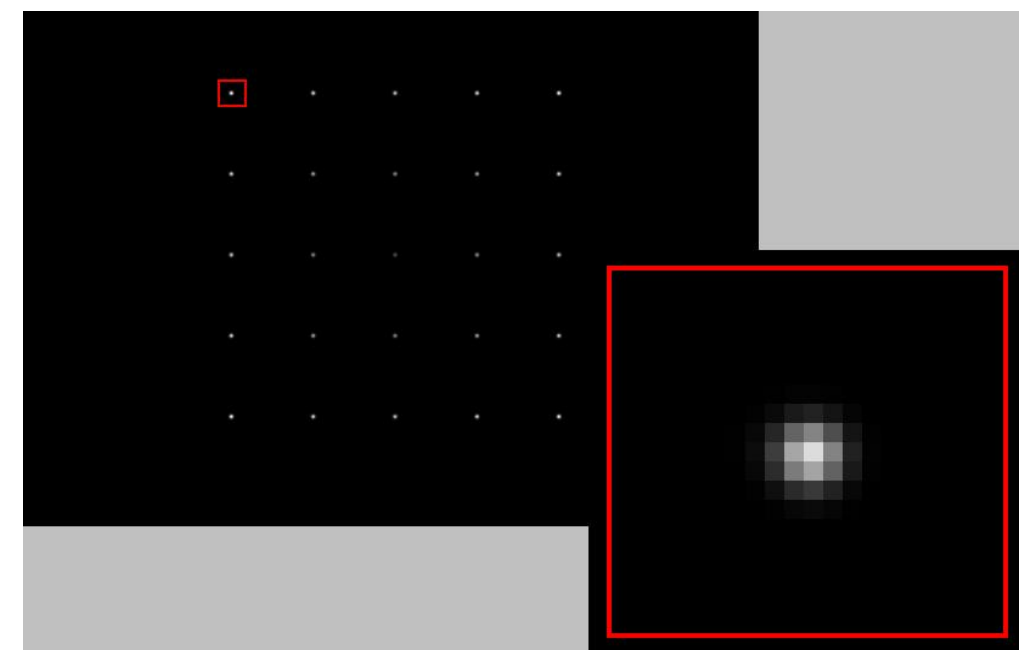

Figure 5.6: Picture of a synthetic calibration image. Note that a simulated PSF is used 


\section{CHAPTER 6: CTIS H MATRIX PROPERTIES}

The previous chapter described that the system H matrix for a CTIS is formed from calibration images. This chapter will continue the analysis of CTIS H matrices and their properties, showing in practice how an $\mathrm{H}$ matrix is formed from calibration images, describing what these system matrices look like, how they can be reformed and manipulated, and finally will describe some of the specific mathematical properties of CTIS H matrices.

\subsection{Size of H matrix}

Perhaps the most significant property of a CTIS H matrix is its size. These systems matrices often contain a huge number of elements. The $\mathrm{H}$ matrix serves as a mapping from object/voxel space to image/pixel space, so the number of elements in a CTIS H matrix is equal to the number of pixels on the focal plane (corresponding to the number of rows in the $\mathrm{H}$ matrix) times the number of voxels in the datacube (the number of columns in the H matrix). For a CTIS with a zero order size of 33 pixels by 33 pixels with 40 spectral bands that uses a focal plane with 480 by 480 pixel resolution, the resulting system $\mathrm{H}$ matrix will contain close to 10 billion total elements $\left(33 \times 33 \times 40 \times 480 \times 480=10.0362 * 10^{10}\right)$. The size of these matrices grow exponentially for systems with higher resolutions. Typical datacube dimensions for a visible CTIS are often around 100 pixels by 100 pixels spatial with 300 spectral bands using a focal plane with 2048 by 2048 resolution, resulting in an $\mathrm{H}$ matrix with more than 12 trillion elements. Due to the sheer size of these matrices, the system H matrices for constructed CTIS systems are relegated to only being stored digitally on a computer. It becomes a nearly impossible task to try and determine the properties or general structure of the matrix just by looking at the various individual elements with so many millions of matrix elements to examine.

In order to get a general visual feel for what the CTIS H matrix is like, this chapter will investigate a "simple representation" of the CTIS system and examine its H matrix. This "simple" CTIS consists of a limited number of diffraction orders, an extremely limited number of spatial and spectral samples, and only considers a strictly discrete to discrete mapping of the object onto the focal plane. Specifically, the example CTIS system will have 9 diffraction orders, a zero order that is 3 pixels x 3 pixels in size, consist of only 3 spectral bands (blue, green, and red), and will have a minimally sized focal plane. Figure 6.1 shows what a CTIS image from such a system would like when viewing a broadband object filling the field of view. 


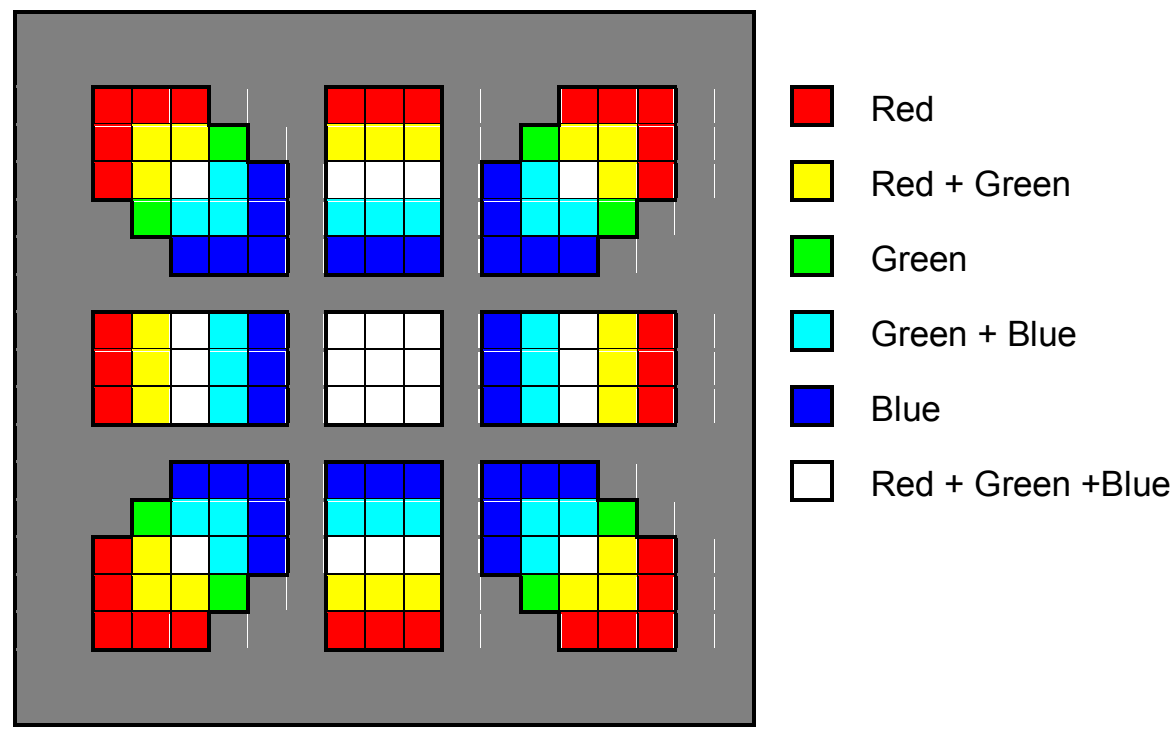

Figure 6.1: Representation of the CTIS image of a uniform and discrete broadband object filling the field of view, for a CTIS system with 3 spectral bands and $3 \times 3$ spatial resolution.

The resulting $\mathrm{H}$ matrix for this example system is extremely small in comparison to $\mathrm{H}$ matrices for constructed CTIS systems. When excluding pixels on the focal plane that do not receive any light, the $\mathrm{H}$ matrix for this simplified system contains less than four thousand elements. This lower resolution example allows one to see the overall structure of the CTIS system $\mathrm{H}$ matrix since the $\mathrm{H}$ matrix can be visually examined with just a few pages of paper. The actual $\mathrm{H}$ matrix for the LWIR CTIS system will have a very similar structure but will be scaled to have more spectral bands, diffraction orders, and zero order spatial positions. An example of a calibration image used to form the H matrix for the LWIR CTIS is shown in figure 6.2. The following pages illustrate the procedure for the formation of the $\mathrm{H}$ matrix with the example CTIS system.

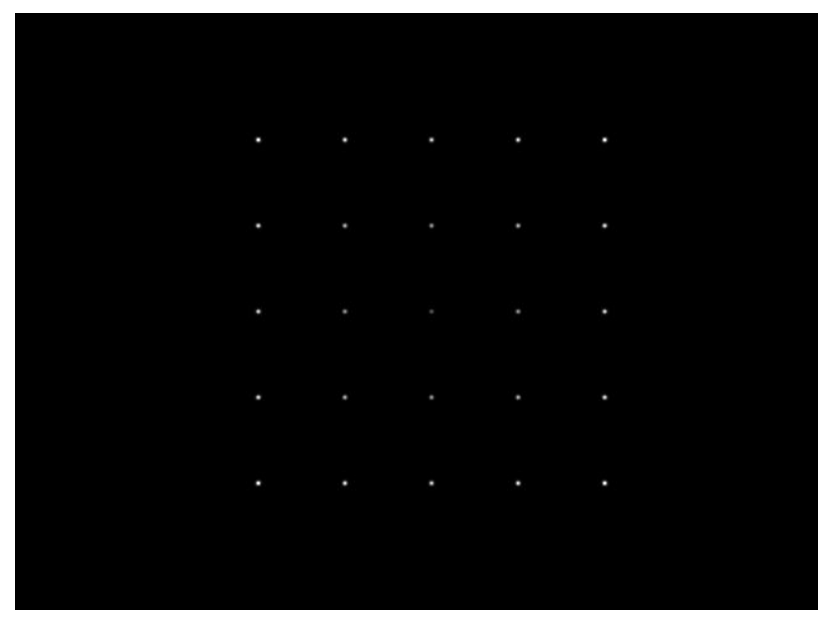

Figure 6.2: Sample calibration image for the LWIR CTIS 


\subsection{Forming the H Matrix}

During the calibration of a CTIS system, a calibration image must be acquired for each voxel in the description of the datacube. As previously described, this is often done by coupling the output of a monochromator into an optical fiber, that is held in a fixed position, and recording images of the fiber as the wavelength output of the monochromator is changed. Due to the shift invariance property of the system, all of the other calibration images needed to form the $\mathrm{H}$ matrix can be generated from this single set of data, simplifying the calibration procedure. Figure 6.3 illustrates the set of calibration images, one taken at each wavelength, with the fiber held at a fixed location.

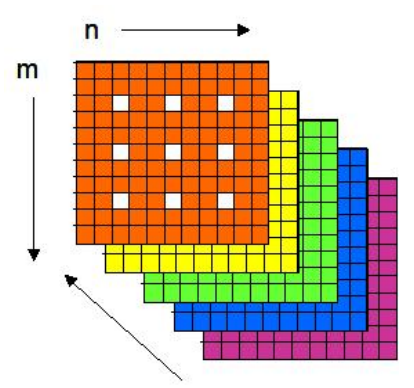

$\lambda$

Figure 6.3: With the point source at a fixed location in the scene, the wavelength of the point source is changed and calibration images are taken at each wavelength.
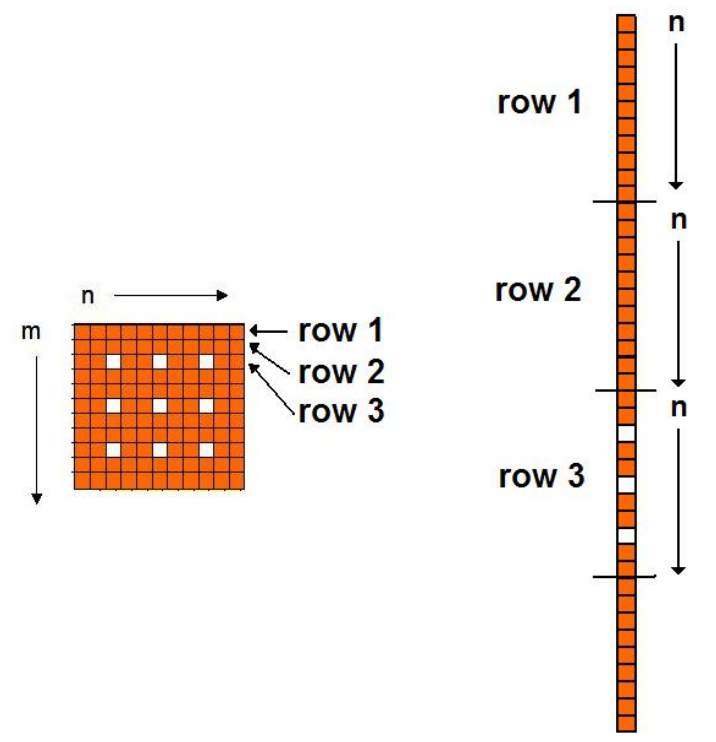

Figure 6.4: Each calibration image can then be represented as a single column vector by a simple rasterization.

When forming the system $\mathrm{H}$ matrix, each column of the $\mathrm{H}$ matrix will be composed of a single calibration image. Each 2-dimensional calibration image is turned into a 1-dimensional column vector by rasterizing the data across the rows of the calibration image, as shown in figure 6.4. The set of calibrations images acquired with the fiber held at a fixed location will form a group 
of columns within the $\mathrm{H}$ matrix. In this example, the calibration image for the highest wavelength of the spectrum is used to form the first column of the $\mathrm{H}$ matrix, and the next column of the $\mathrm{H}$ matrix will contain the data from the calibration image of the same point object in the same spatial position, but with a slightly shorter wavelength. This continues until the shortest wavelength is reached, where the next column of the $\mathrm{H}$ matrix will be an image of a point object composed of the longest wavelength at the next spatial location in the zero order, as illustrated in figures 6.5 and 6.6 .
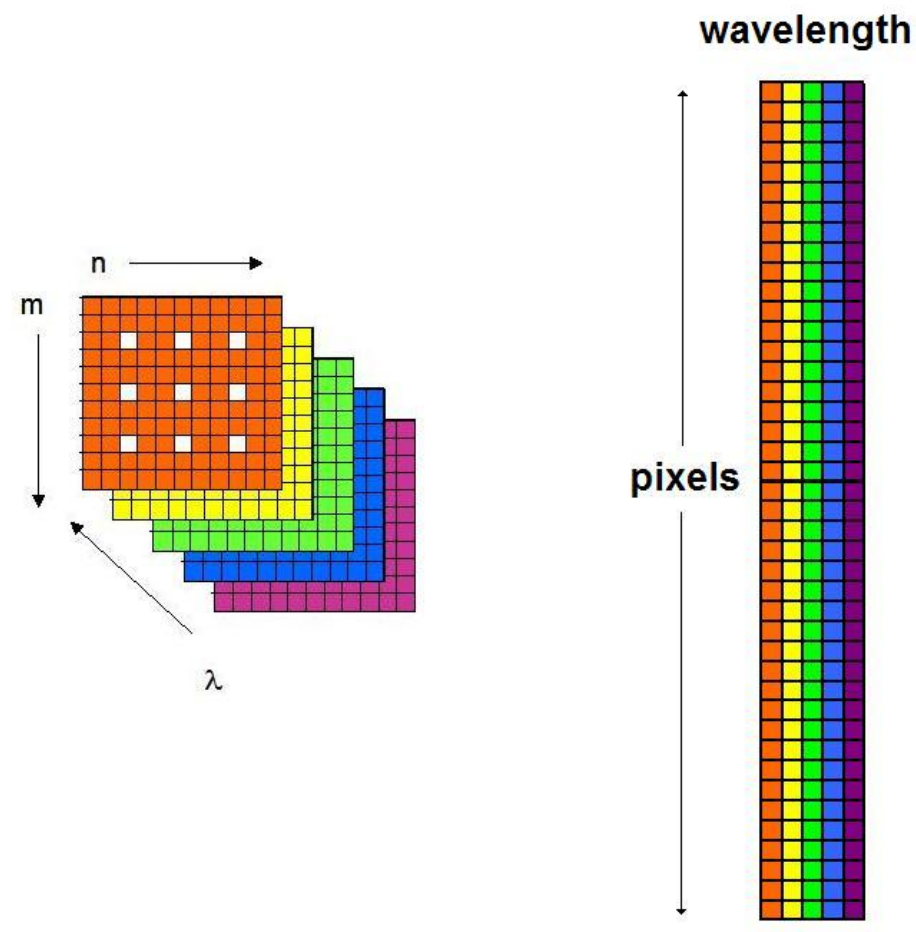

Figure 6.5: With the monochromatic point source at a fixed location in the scene and describing each calibration image as a column vector, the set of calibration images can be combined to form a matrix of column vectors. 

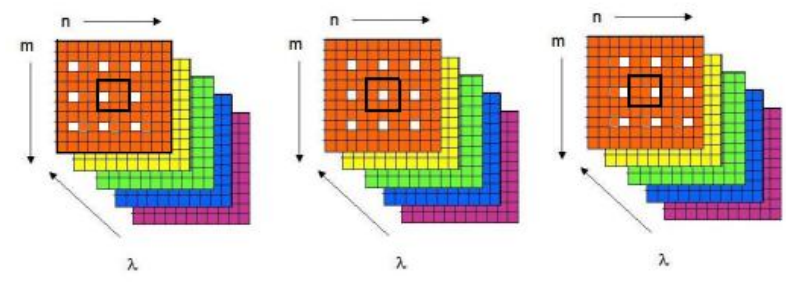

\section{Zero Order consists of 9 spatial positions}

Data is taken at each wavelength at each spatial position to form the H Matrix.

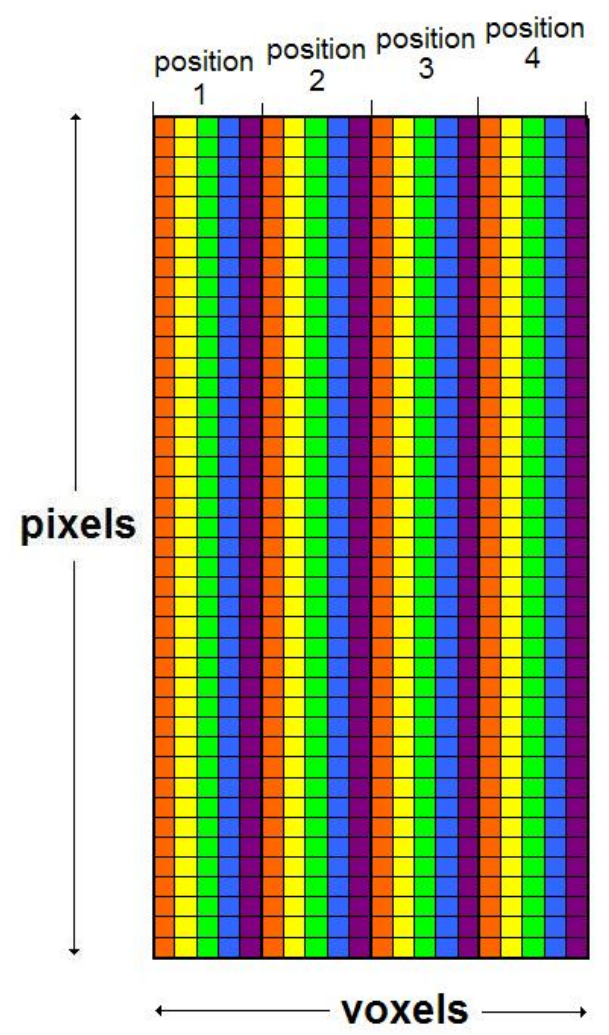

Figure 6.6: The H matrix of the CTIS system is made from many sets of column vectors. Each set is composed of the images of each wavelength at a given spatial position. The number of spatial positions is defined by the size of the zero order on the focal plane array.

Once all of the calibration images for all of the spatial locations are incorporated into the columns of the matrix, the newly formed CTIS H matrix gives us a mathematical operator describing the system, relating the object/voxel space to the image/pixel space as illustrated in figure 6.7 by the standard imaging equation. In this equation the object datacube is represented with a 1D column vector. The order of the datacube voxel elements in the $1 \mathrm{D}$ vector is given by the order of voxel calibration images used to make the $\mathrm{H}$ matrix. In this example, the first element of the datacube vector describes the irradiance for the highest wavelength band at position 1. The next element in the vector describes the irradiance at position 1 for the next lower wavelength band, and so on. Similarly, the elements in the 1D image vector, g, are related to the order of the pixels values that form the rows of the H matrix. In this example, the first element of the image vector corresponds to the top left corner pixel, the subsequent element corresponds to the next pixel to the right, and so on in the same type of raster scan used to vectorize the calibration images. 

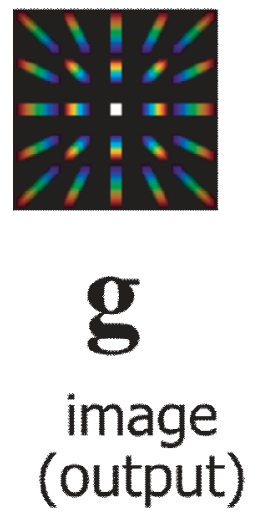

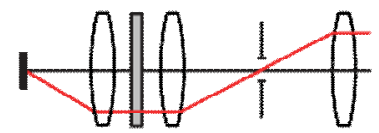

H

system operator

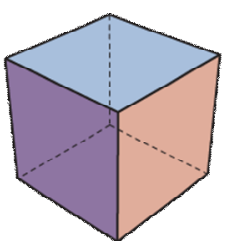

$\mathbf{f}(x, y, \lambda)$

object

(input)

Figure 6.7: One dimensional vectors are used to describe both the object datacube, $f$, and the recorded CTIS image, $\mathrm{g}$, while the system $\mathrm{H}$ operator is given by a matrix. The order of the vector elements describing the voxels in the object vector and pixels in the image vectors must

be in the same order as given in the columns and rows of the $\mathrm{H}$ matrix.

Relating back to the specific example of the CTIS with 9 diffraction orders, a 3x3 zero order, and 3 wavelength bands, consider what the calibration images for such a system would look like. A few examples are shown in figure 6.8.

Wavelength 1
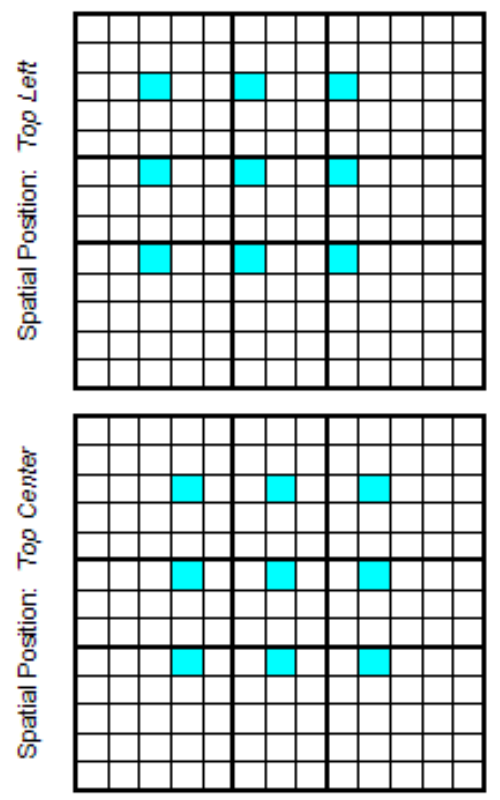

Wavelength 2
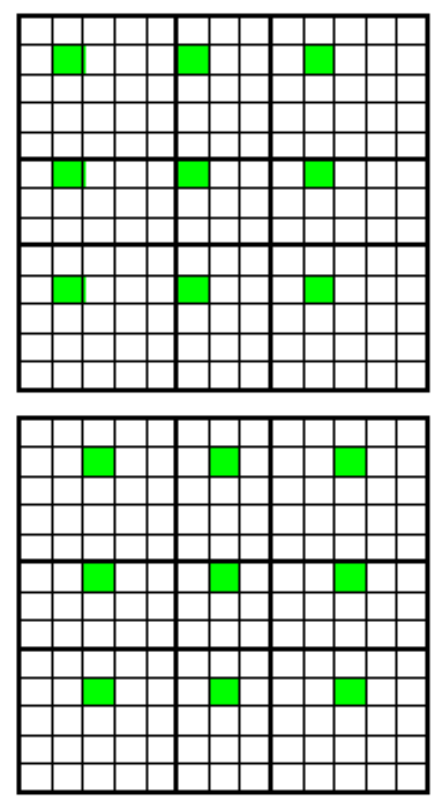

Wavelength 3
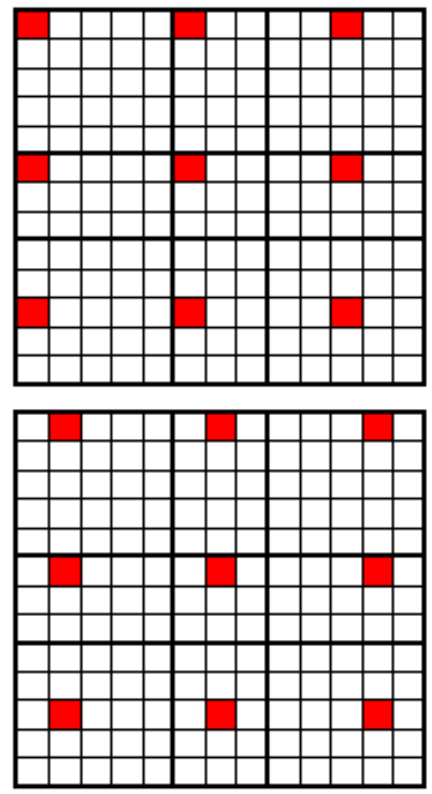

Figure 6.8: Various calibration images for the example CTIS system

The H matrix for this CTIS system will be constructed in a similar fashion described above by turning each of the calibration images into a column of the $\mathrm{H}$ matrix. The corresponding $\mathrm{H}$ matrix created for the example $3 \times 3$ CTIS system is shown on the following pages illustrating some of the various sets of calibration images, row/pixel locations, and the signal locations of the various diffraction orders. Note that in this example, all of the pixels forming the various 
calibration images have values of either zero or one. For the pixels that have a value of one, the matrix element is colored to represent the wavelength band for that calibration image. From the matrix representation, it is clear which rows of the matrix correspond to pixels in the zero diffraction order. In these rows, the same pixels remain lit when scanning through the various wavelengths. Since the raster scan of the pixels was from left to right, the horizontal orders to the left and right of the zero order are easily identifiable as the pixel separation increases at higher wavelengths. The matrix elements for the remaining orders do seem to have a diagonal like structure for each wavelength, but due to the different wavelengths mapping to different rows, the raster scan vectorization of the images, and the order of calibration images these nonzero matrix elements are somewhat interspersed.

Columns corresponding to calibration images for lit Top Left pixel of zero order

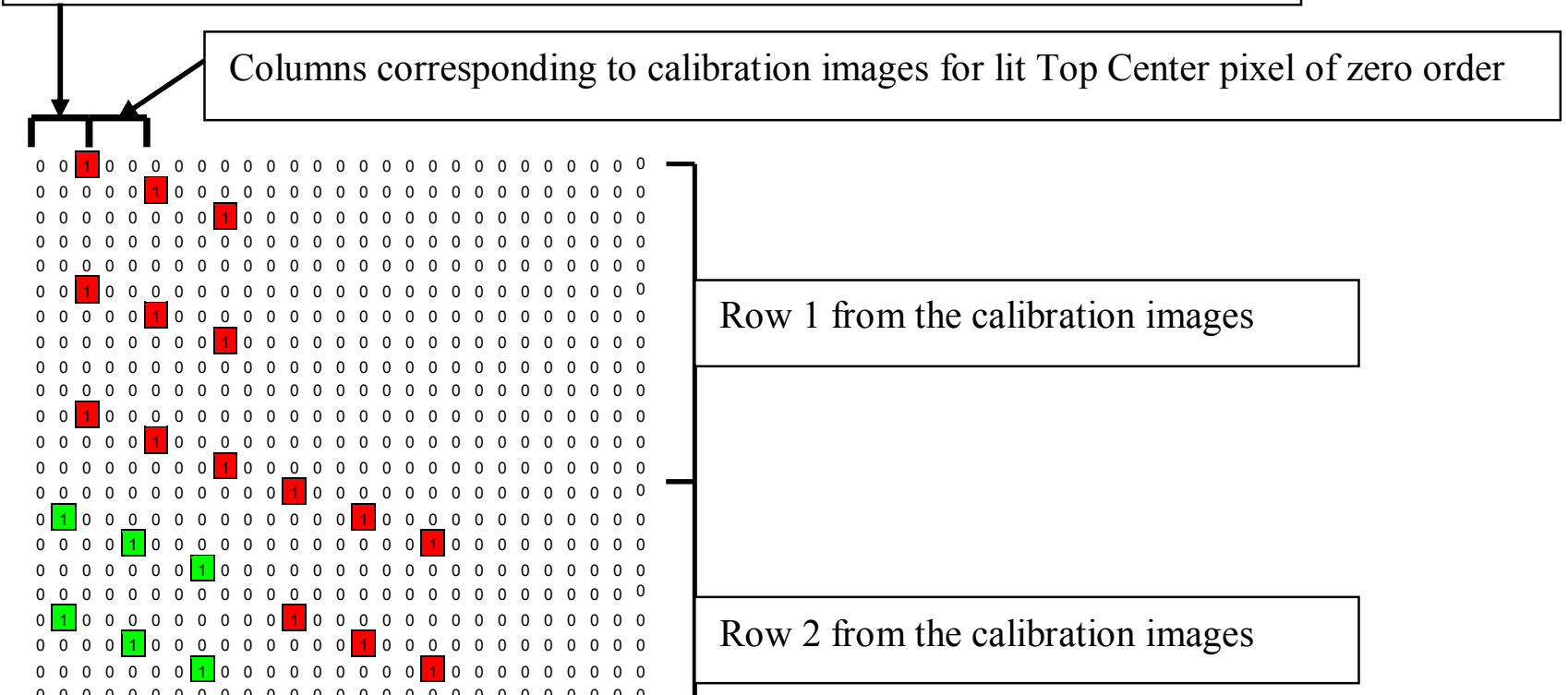
$100 \begin{array}{lllllllllll}1 & 0 & 0 & 0 & 0 & 0 & 0 & 0 & 0\end{array}$ $0 \begin{array}{lllllllllllllllllllllllllll}0 & 0 & 0 & 0 & 0 & 0 & 0 & 0 & 0 & 0 & 0 & 0 & 0 & 0 & 0 & 0 & 0 & 0 & 0 & 0 & 0 & 0 & 0 & 0 & 0 & 0\end{array}$

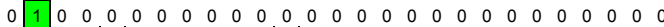

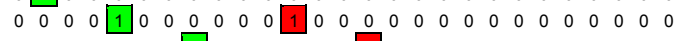
$\begin{array}{lllllllllllllllllllllllllll}0 & 0 & 0 & 0 & 0 & 0 & 0 & 1 & 0 & 0 & 0 & 0 & 0 & 0 & 1 & 0 & 0 & 0 & 0 & 0 & 0 & 0 & 0 & 0 & 0 & 0 & 0\end{array}$ $\begin{array}{lllllllllllllllllllllllllll}0 & 0 & 0 & 0 & 0 & 0 & 0 & 0 & 0 & 0 & 0 & 0 & 0 & 0 & 0 & 0 & 0 & 1 & 0 & 0 & 0 & 0 & 0 & 0 & 0 & 0 & 0\end{array}$ $\begin{array}{lllllllllllllllllllllllllll}0 & 0 & 0 & 0 & 0 & 0 & 0 & 0 & 0 & 0 & 0 & 0 & 0 & 0 & 0 & 0 & 0 & 0 & 0 & 0 & 1 & 0 & 0 & 0 & 0 & 0 & 0\end{array}$

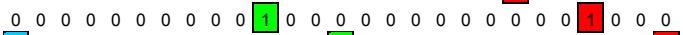

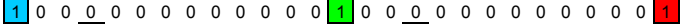

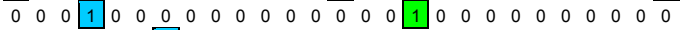

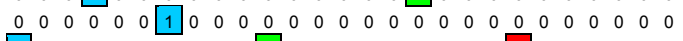
$\begin{array}{lllllllllllllllllllllllllll}1 & 0 & 0 & 0 & 0 & 0 & 0 & 0 & 0 & 0 & 1 & 0 & 0 & 0 & 0 & 0 & 0 & 0 & 0 & 0 & 1 & 0 & 0 & 0 & 0 & 0 & 0\end{array}$ $\begin{array}{lllllllllllllllllllllllllll}0 & 0 & 0 & 1 & 0 & 0 & 0 & 0 & 0 & 0 & 0 & 0 & 0 & 1 & 0 & 0 & 0 & 0 & 0 & 0 & 0 & 0 & 0 & 1 & 0 & 0 & 0\end{array}$ $\begin{array}{lllllllllllllllllllllllllll}0 & 0 & 0 & 0 & 0 & 0 & 1 & 0 & 0 & 0 & 0 & 0 & 0 & 0 & 0 & 0 & 1 & 0 & 0 & 0 & 0 & 0 & 0 & 0 & 0 & 0 & 1\end{array}$

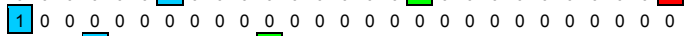
$\begin{array}{llllllllllllllllllllllllllll}0 & 0 & 0 & 1 & 0 & 0 & 0 & 0 & 0 & 0 & 1 & 0 & 0 & 0 & 0 & 0 & 0 & 0 & 0 & 0 & 0 & 0 & 0 & 0 & 0 & 0 & 0\end{array}$ $\begin{array}{lllllllllllllllllllllllllll}0 & 0 & 0 & 0 & 0 & 0 & 1 & 0 & 0 & 0 & 0 & 0 & 0 & 1 & 0 & 0 & 0 & 0 & 0 & 0 & 1 & 0 & 0 & 0 & 0 & 0 & 0\end{array}$ $\begin{array}{llllllllllllllllllllllllllll}0 & 0 & 0 & 0 & 0 & 0 & 0 & 0 & 0 & 0 & 0 & 0 & 0 & 0 & 0 & 0 & 1 & 0 & 0 & 0 & 0 & 0 & 0 & 1 & 0 & 0 & 0\end{array}$ $\begin{array}{lllllllllllllllllllllllllll}0 & 0 & 0 & 0 & 0 & 0 & 0 & 0 & 0 & 0 & 0 & 0 & 0 & 0 & 0 & 0 & 0 & 0 & 0 & 0 & 0 & 0 & 0 & 0 & 0 & 0 & 1\end{array}$ $\begin{array}{lllllllllllllllllllllllllll}0 & 0 & 0 & 0 & 0 & 0 & 0 & 0 & 0 & 0 & 0 & 0 & 0 & 0 & 0 & 0 & 0 & 0 & 0 & 0 & 0 & 0 & 0 & 0 & 0 & 0 & 0\end{array}$ $\begin{array}{lllllllllllllllllllllllllll}0 & 0 & 0 & 0 & 0 & 0 & 0 & 0 & 0 & 0 & 0 & 0 & 0 & 0 & 0 & 0 & 0 & 0 & 0 & 1 & 0 & 0 & 0 & 0 & 0 & 0 & 0\end{array}$ $\begin{array}{lllllllllllllllllllllllllll}0 & 0 & 0 & 0 & 0 & 0 & 0 & 0 & 0 & 1 & 0 & 0 & 0 & 0 & 0 & 0 & 0 & 0 & 0 & 0 & 0 & 0 & 1 & 0 & 0 & 0 & 0\end{array}$ $\begin{array}{lllllllllllllllllllllllllll}0 & 0 & 0 & 0 & 0 & 0 & 0 & 0 & 0 & 0 & 0 & 0 & 1 & 0 & 0 & 0 & 0 & 0 & 0 & 0 & 0 & 0 & 0 & 0 & 0 & 1 & 0\end{array}$ $\begin{array}{lllllllllllllllllllllllllll}0 & 0 & 0 & 0 & 0 & 0 & 0 & 0 & 0 & 0 & 0 & 0 & 0 & 0 & 0 & 1 & 0 & 0 & 0 & 0 & 0 & 0 & 0 & 0 & 0 & 0 & 0\end{array}$ $\begin{array}{lllllllllllllllllllllllllll}0 & 0 & 0 & 0 & 0 & 0 & 0 & 0 & 0 & 1 & 0 & 0 & 0 & 0 & 0 & 0 & 0 & 0 & 0 & 1 & 0 & 0 & 0 & 0 & 0 & 0 & 0\end{array}$ $\begin{array}{llllllllllllllllllllllllllll}0 & 0 & 0 & 0 & 0 & 0 & 0 & 0 & 0 & 0 & 0 & 0 & 1 & 0 & 0 & 0 & 0 & 0 & 0 & 0 & 0 & 0 & 1 & 0 & 0 & 0 & 0\end{array}$ $\begin{array}{llllllllllllllllllllllllllll}0 & 0 & 0 & 0 & 0 & 0 & 0 & 0 & 0 & 0 & 0 & 0 & 0 & 0 & 0 & 1 & 0 & 0 & 0 & 0 & 0 & 0 & 0 & 0 & 0 & 1 & 0\end{array}$ $\begin{array}{lllllllllllllllllllllllllll}0 & 0 & 0 & 0 & 0 & 0 & 0 & 0 & 0 & 1 & 0 & 0 & 0 & 0 & 0 & 0 & 0 & 0 & 0 & 0 & 0 & 0 & 0 & 0 & 0 & 0 & 0\end{array}$ $\begin{array}{lllllllllllllllllllllllllll}0 & 0 & 0 & 0 & 0 & 0 & 0 & 0 & 0 & 0 & 0 & 0 & 1 & 0 & 0 & 0 & 0 & 0 & 0 & 1 & 0 & 0 & 0 & 0 & 0 & 0 & 0\end{array}$ $\begin{array}{lllllllllllllllllllllllllll}0 & 0 & 0 & 0 & 0 & 0 & 0 & 0 & 0 & 0 & 0 & 0 & 0 & 0 & 0 & 1 & 0 & 0 & 0 & 0 & 0 & 0 & 1 & 0 & 0 & 0 & 0\end{array}$ 


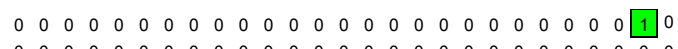
$\begin{array}{lllllllllllllllllllllllllll}0 & 0 & 0 & 0 & 0 & 0 & 0 & 0 & 0 & 0 & 0 & 0 & 0 & 0 & 0 & 0 & 0 & 0 & 0 & 0 & 0 & 0 & 0 & 0 & 0 & 0 & 0\end{array}$ $\begin{array}{llllllllllllllllllllllllllllll}0 & 0 & 0 & 0 & 0 & 0 & 0 & 0 & 0 & 0 & 0 & 0 & 0 & 0 & 0 & 0 & 0 & 0 & 0 & 0 & 0 & 0 & 0 & 0 & 0 & 0 & 0\end{array}$ $\begin{array}{lllllllllllllllllllllllllll}0 & 0 & 0 & 0 & 0 & 0 & 0 & 0 & 0 & 0 & 0 & 0 & 0 & 0 & 0 & 0 & 0 & 0 & 0 & 0 & 0 & 0 & 0 & 0 & 0 & 0 & 0\end{array}$ $\begin{array}{lllllllllllllllllllllllllll}0 & 0 & 0 & 0 & 0 & 0 & 0 & 0 & 0 & 0 & 0 & 0 & 0 & 0 & 0 & 0 & 0 & 0 & 1 & 0 & 0 & 0 & 0 & 0 & 0 & 0 & 0\end{array}$

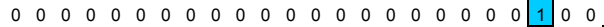

$\begin{array}{llllllllllllllllllllllllll}0 & 0 & 0 & 0 & 0 & 0 & 0 & 0 & 0 & 0 & 0 & 0 & 0 & 0 & 0 & 0 & 0 & 0 & 0 & 0 & 0 & 0 & 0 & 0\end{array}$

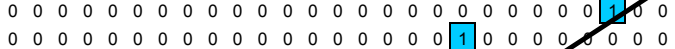

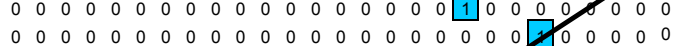

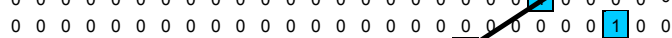
$\begin{array}{llllllllllllllllllllllllllllllllll}0 & 0 & 0 & 0 & 0 & 0 & 0 & 0 & 0 & 0 & 0 & 0 & 0 & 0 & 0 & 0 & 0 & 0 & 0 & 0 & 0 & 0 & 0 & 0 & 0\end{array}$

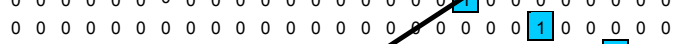
$\begin{array}{llllllllllllllllllllllllllllllllll}0 & 0 & 0 & 0 & 0 & 0 & 0 & 0 & 0 & 0 & 0 & 0 & 0 & 0 & 0 & 0 & 0 & 0 & 0 & 0 & 0 & 0 & 0 & 0 & 1 & 0 & 0\end{array}$

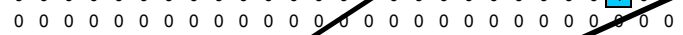

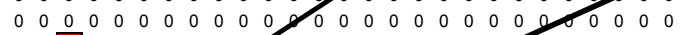
$\begin{array}{llllllllllllllllllllllllll}0 & 0 & 1 & 0 & 0 & 0 & 0 & 0 & 0 & 0 & 0 & 0 & 0 & 0 & 0 & 0 & 0 & 0 & 0 & 0 & 0 & 0 & 0 & 0 & 0 & 0\end{array}$ $\begin{array}{llllllllllllllllllllllllll}0 & 1 & 0 & 0 & 0 & 1 & 0 & 0 & 0 & 0 & 0 & 0 & 0 & 0 & 0 & 0 & 0 & 0 & 0 & 0 & 0 & 0 & 0 & 0 & 0\end{array}$ $\begin{array}{lllllllllllllllllllllllllll}1 & 0 & 0 & 0 & 1 & 0 & 0 & 0 & 1 & 0 & 0 & 0 & 0 & 0 & 0 & 0 & 0 & 0 & 0 & 0 & 0 & 0 & 0 & 0 & 0 & 0\end{array}$

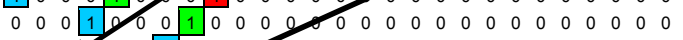
$\begin{array}{llllllllllllllllllllllll}0 & 0 & 0 & 0 & 0 & 1 & 0 & 0 & 0 & 0 & 0 & 0 & 0 & 0 & 0 & 0 & 0 & 0 & 0 & 0 & 0 & 0 & 0 & 0\end{array}$ $\begin{array}{llllllllllllllllllllllllll}1 & 1 & 1 & 0 & 0 & 0 & 0 & 0 & 0 & 0 & 0 & 0 & 0 & 0 & 0 & 0 & 0 & 0 & 0 & 0 & 0 & 0 & 0 & 0 & 0\end{array}$ $\begin{array}{llllllllllllllllllllllllllll}0 & 0 & 0 & 1 & 1 & 1 & 0 & 0 & 0 & 0 & 0 & 0 & 0 & 0 & 0 & 0 & 0 & 0 & 0 & 0 & 0 & 0 & 0 & 0 & 0 & 0 & 0\end{array}$ \begin{tabular}{lllllll|l|lllllllllllllllllll}
0 & 0 & 0 & 0 & 0 & 0 & 1 & 1 & 1 & 0 & 0 & 0 & 0 & 0 & 0 & 0 & 0 & 0 & 0 & 0 & 0 & 0 & 0 & 0 & 0 & 0 & 0
\end{tabular}

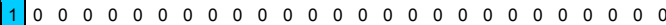
$\begin{array}{llllllllllllllllllllllllllll}0 & 1 & 0 & 1 & 0 & 0 & 0 & 0 & 0 & 0 & 0 & 0 & 0 & 0 & 0 & 0 & 0 & 0 & 0 & 0 & 0 & 0 & 0 & 0 & 0 & 0 & 0\end{array}$ $\begin{array}{llllllllllllllllllllllllllll}0 & 0 & 1 & 0 & 1 & 0 & 1 & 0 & 0 & 0 & 0 & 0 & 0 & 0 & 0 & 0 & 0 & 0 & 0 & 0 & 0 & 0 & 0 & 0 & 0 & 0 & 0\end{array}$ $\begin{array}{lllllllllllllllllllllllllll}0 & 0 & 0 & 0 & 0 & 1 & 0 & 1 & 0 & 0 & 0 & 0 & 0 & 0 & 0 & 0 & 0 & 0 & 0 & 0 & 0 & 0 & 0 & 0 & 0 & 0 & 0\end{array}$ $\begin{array}{lllllllllllllllllllllllllll}0 & 0 & 0 & 0 & 0 & 0 & 0 & 0 & 1 & 0 & 0 & 0 & 0 & 0 & 0 & 0 & 0 & 0 & 0 & 0 & 0 & 0 & 0 & 0 & 0 & 0 & 0\end{array}$ $\begin{array}{lllllllllllllllllllllllllll}0 & 0 & 0 & 0 & 0 & 0 & 0 & 0 & 0 & 0 & 0 & 1 & 0 & 0 & 0 & 0 & 0 & 0 & 0 & 0 & 0 & 0 & 0 & 0 & 0 & 0 & 0\end{array}$ $\begin{array}{llllllllllllllllllllllllllll}0 & 0 & 0 & 0 & 0 & 0 & 0 & 0 & 0 & 0 & 1 & 0 & 0 & 0 & 1 & 0 & 0 & 0 & 0 & 0 & 0 & 0 & 0 & 0 & 0 & 0 & 0\end{array}$ $\begin{array}{llllllllllllllllllllllllllll}0 & 0 & 0 & 0 & 0 & 0 & 0 & 0 & 0 & 1 & 0 & 0 & 0 & 1 & 0 & 0 & 0 & 1 & 0 & 0 & 0 & 0 & 0 & 0 & 0 & 0 & 0\end{array}$ $\begin{array}{lllllllllllllllllllllllllll}0 & 0 & 0 & 0 & 0 & 0 & 0 & 0 & 0 & 0 & 0 & 0 & 1 & 0 & 0 & 0 & 1 & 0 & 0 & 0 & 0 & 0 & 0 & 0 & 0 & 0 & 0\end{array}$ $\begin{array}{lllllllllllllllllllllllllll}0 & 0 & 0 & 0 & 0 & 0 & 0 & 0 & 0 & 0 & 0 & 0 & 0 & 0 & 0 & 1 & 0 & 0 & 0 & 0 & 0 & 0 & 0 & 0 & 0 & 0 & 0\end{array}$

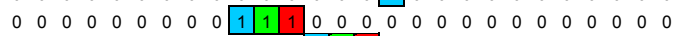
\begin{tabular}{lllllllllllll|lllllllllllllll}
0 & 0 & 0 & 0 & 0 & 0 & 0 & 0 & 0 & 0 & 0 & 0 & 1 & 1 & 1 & 0 & 0 & 0 & 0 & 0 & 0 & 0 & 0 & 0 & 0 & 0 & 0
\end{tabular} \begin{tabular}{llllllllllllllll|l|l|lllllllll}
0 & 0 & 0 & 0 & 0 & 0 & 0 & 0 & 0 & 0 & 0 & 0 & 0 & 0 & 0 & 1 & 1 & 1 & 0 & 0 & 0 & 0 & 0 & 0 & 0 & 0 & 0
\end{tabular} $\begin{array}{lllllllllllllllllllllllllll}0 & 0 & 0 & 0 & 0 & 0 & 0 & 0 & 0 & 1 & 0 & 0 & 0 & 0 & 0 & 0 & 0 & 0 & 0 & 0 & 0 & 0 & 0 & 0 & 0 & 0 & 0\end{array}$ $\begin{array}{llllllllllllllllllllllllllll}0 & 0 & 0 & 0 & 0 & 0 & 0 & 0 & 0 & 0 & 1 & 0 & 1 & 0 & 0 & 0 & 0 & 0 & 0 & 0 & 0 & 0 & 0 & 0 & 0 & 0 & 0\end{array}$ $\begin{array}{lllllllllllllllllllllllllllll}0 & 0 & 0 & 0 & 0 & 0 & 0 & 0 & 0 & 0 & 0 & 1 & 0 & 1 & 0 & 1 & 0 & 0 & 0 & 0 & 0 & 0 & 0 & 0 & 0 & 0 & 0\end{array}$ $\begin{array}{llllllllllllllllllllllllllll}0 & 0 & 0 & 0 & 0 & 0 & 0 & 0 & 0 & 0 & 0 & 0 & 0 & 0 & 1 & 0 & 1 & 0 & 0 & 0 & 0 & 0 & 0 & 0 & 0 & 0 & 0\end{array}$ $\begin{array}{lllllllllllllllllllllllllll}0 & 0 & 0 & 0 & 0 & 0 & 0 & 0 & 0 & 0 & 0 & 0 & 0 & 0 & 0 & 0 & 0 & 1 & 0 & 0 & 0 & 0 & 0 & 0 & 0 & 0 & 0\end{array}$ $\begin{array}{lllllllllllllllllllllllllll}0 & 0 & 0 & 0 & 0 & 0 & 0 & 0 & 0 & 0 & 0 & 0 & 0 & 0 & 0 & 0 & 0 & 0 & 0 & 0 & 1 & 0 & 0 & 0 & 0 & 0 & 0\end{array}$ $\begin{array}{lllllllllllllllllllllllllll}0 & 0 & 0 & 0 & 0 & 0 & 0 & 0 & 0 & 0 & 0 & 0 & 0 & 0 & 0 & 0 & 0 & 0 & 0 & 1 & 0 & 0 & 0 & 1 & 0 & 0 & 0\end{array}$ \begin{tabular}{llllllllllllllllllllllllllll}
0 & 0 & 0 & 0 & 0 & 0 & 0 & 0 & 0 & 0 & 0 & 0 & 0 & 0 & 0 & 0 & 0 & 0 & 1 & 0 & 0 & 0 & 1 & 0 & 0 & 0 & 1 \\
\hline
\end{tabular} $\begin{array}{llllllllllllllllllllllllllll}0 & 0 & 0 & 0 & 0 & 0 & 0 & 0 & 0 & 0 & 0 & 0 & 0 & 0 & 0 & 0 & 0 & 0 & 0 & 0 & 0 & 1 & 0 & 0 & 0 & 1 & 0\end{array}$ $\begin{array}{llllllllllllllllllllllllllll}0 & 0 & 0 & 0 & 0 & 0 & 0 & 0 & 0 & 0 & 0 & 0 & 0 & 0 & 0 & 0 & 0 & 0 & 0 & 0 & 0 & 0 & 0 & 0 & 1 & 0 & 0\end{array}$ $\begin{array}{llllllllllllllllllllllllllll}0 & 0 & 0 & 0 & 0 & 0 & 0 & 0 & 0 & 0 & 0 & 0 & 0 & 0 & 0 & 0 & 0 & 0 & 1 & 1 & 1 & 0 & 0 & 0 & 0 & 0 & 0\end{array}$ \begin{tabular}{llllllllllllllllllllllll|l|l|lll}
0 & 0 & 0 & 0 & 0 & 0 & 0 & 0 & 0 & 0 & 0 & 0 & 0 & 0 & 0 & 0 & 0 & 0 & 0 & 0 & 0 & 1 & 1 & 1 & 0 & 0 & 0 \\
\hline
\end{tabular}

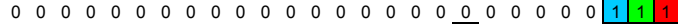
$\begin{array}{lllllllllllllllllllllllllll}0 & 0 & 0 & 0 & 0 & 0 & 0 & 0 & 0 & 0 & 0 & 0 & 0 & 0 & 0 & 0 & 0 & 0 & 1 & 0 & 0 & 0 & 0 & 0 & 0 & 0 & 0\end{array}$ $\begin{array}{lllllllllllllllllllllllllllllll}0 & 0 & 0 & 0 & 0 & 0 & 0 & 0 & 0 & 0 & 0 & 0 & 0 & 0 & 0 & 0 & 0 & 0 & 0 & 1 & 0 & 1 & 0 & 0 & 0 & 0 & 0\end{array}$ $\begin{array}{llllllllllllllllllllllllllll}0 & 0 & 0 & 0 & 0 & 0 & 0 & 0 & 0 & 0 & 0 & 0 & 0 & 0 & 0 & 0 & 0 & 0 & 0 & 0 & 1 & 0 & 1 & 0 & 1 & 0 & 0\end{array}$ $\begin{array}{lllllllllllllllllllllllllll}0 & 0 & 0 & 0 & 0 & 0 & 0 & 0 & 0 & 0 & 0 & 0 & 0 & 0 & 0 & 0 & 0 & 0 & 0 & 0 & 0 & 0 & 0 & 1 & 0 & 1 & 0\end{array}$ $\begin{array}{lllllllllllllllllllllllllll}0 & 0 & 0 & 0 & 0 & 0 & 0 & 0 & 0 & 0 & 0 & 0 & 0 & 0 & 0 & 0 & 0 & 0 & 0 & 0 & 0 & 0 & 0 & 0 & 0 & 0 & 1\end{array}$ $\begin{array}{lllllllllllllllllllllllllll}0 & 0 & 0 & 0 & 0 & 0 & 0 & 0 & 0 & 0 & 0 & 0 & 0 & 0 & 0 & 0 & 0 & 0 & 0 & 0 & 0 & 0 & 0 & 0 & 0 & 0 & 0\end{array}$ $0 \begin{array}{lllllllllllllllllllllllllll}0 & 0 & 0 & 0 & 0 & 0 & 0 & 0 & 0 & 0 & 0 & 0 & 0 & 0 & 0 & 0 & 0 & 0 & 0 & 0 & 0 & 0 & 0 & 0 & 0 & 0 & 0\end{array}$

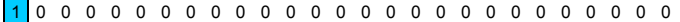

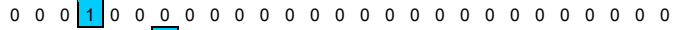

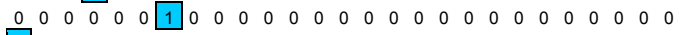

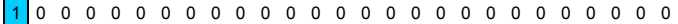
$\begin{array}{lllllllllllllllllllllllllll}0 & 0 & 0 & 1 & 0 & 0 & 0 & 0 & 0 & 0 & 0 & 0 & 0 & 0 & 0 & 0 & 0 & 0 & 0 & 0 & 0 & 0 & 0 & 0 & 0 & 0 & 0\end{array}$ $\begin{array}{lllllllllllllllllllllllllll}0 & 0 & 0 & 0 & 0 & 0 & 1 & 0 & 0 & 0 & 0 & 0 & 0 & 0 & 0 & 0 & 0 & 0 & 0 & 0 & 0 & 0 & 0 & 0 & 0 & 0 & 0\end{array}$

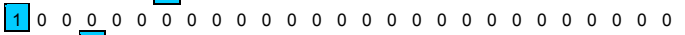
$\begin{array}{lllllllllllllllllllllllllll}0 & 0 & 0 & 1 & 0 & 0 & 0 & 0 & 0 & 0 & 0 & 0 & 0 & 0 & 0 & 0 & 0 & 0 & 0 & 0 & 0 & 0 & 0 & 0 & 0 & 0 & 0\end{array}$

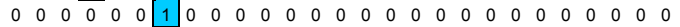
$\begin{array}{lllllllllllllllllllllllllll}0 & 0 & 0 & 0 & 0 & 0 & 0 & 0 & 0 & 0 & 0 & 0 & 0 & 0 & 0 & 0 & 0 & 0 & 0 & 0 & 0 & 0 & 0 & 0 & 0 & 0 & 0\end{array}$ $\begin{array}{lllllllllllllllllllllllllll}0 & 0 & 0 & 0 & 0 & 0 & 0 & 0 & 0 & 0 & 0 & 0 & 0 & 0 & 0 & 0 & 0 & 0 & 0 & 0 & 0 & 0 & 0 & 0 & 0 & 0 & 0\end{array}$ $\begin{array}{lllllllllllllllllllllllllll}0 & 0 & 0 & 0 & 0 & 0 & 0 & 0 & 0 & 0 & 0 & 0 & 0 & 0 & 0 & 0 & 0 & 0 & 0 & 0 & 0 & 0 & 0 & 0 & 0 & 0 & 0\end{array}$ $0 \begin{array}{lllllllllllllllllllllllllll}0 & 0 & 0 & 0 & 0 & 0 & 0 & 0 & 0 & 0 & 0 & 0 & 0 & 0 & 0 & 0 & 0 & 0 & 0 & 0 & 0 & 0 & 0 & 0 & 0 & 0\end{array}$

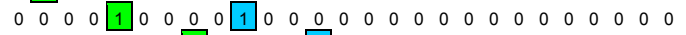

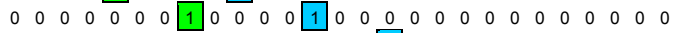
$\begin{array}{lllllllllllllllllllllllllll}0 & 0 & 0 & 0 & 0 & 0 & 0 & 0 & 0 & 0 & 0 & 0 & 0 & 0 & 0 & 1 & 0 & 0 & 0 & 0 & 0 & 0 & 0 & 0 & 0 & 0 & 0\end{array}$ $0 \begin{array}{llllllllllllllllllllllllll}0 & 0 & 0 & 0 & 0 & 0 & 0 & 0 & 1 & 0 & 0 & 0 & 0 & 0 & 0 & 0 & 0 & 0 & 0 & 0 & 0 & 0 & 0 & 0 & 0 & 0\end{array}$

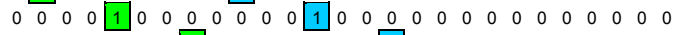
$\begin{array}{lllllllllllllllllllllllllll}0 & 0 & 0 & 0 & 0 & 0 & 0 & 1 & 0 & 0 & 0 & 0 & 0 & 0 & 0 & 1 & 0 & 0 & 0 & 0 & 0 & 0 & 0 & 0 & 0 & 0 & 0\end{array}$ $\begin{array}{lllllllllllllllllllllllllll}0 & 0 & 0 & 0 & 0 & 0 & 0 & 0 & 0 & 1 & 0 & 0 & 0 & 0 & 0 & 0 & 0 & 0 & 0 & 0 & 0 & 0 & 0 & 0 & 0 & 0 & 0\end{array}$

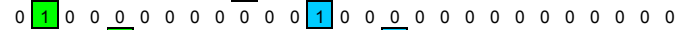

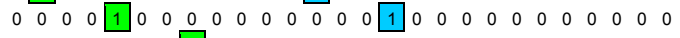
$\begin{array}{lllllllllllllllllllllllllll}0 & 0 & 0 & 0 & 0 & 0 & 0 & 1 & 0 & 0 & 0 & 0 & 0 & 0 & 0 & 0 & 0 & 0 & 0 & 0 & 0 & 0 & 0 & 0 & 0 & 0 & 0\end{array}$ $\begin{array}{lllllllllllllllllllllllllll}0 & 0 & 0 & 0 & 0 & 0 & 0 & 0 & 0 & 0 & 0 & 0 & 0 & 0 & 0 & 0 & 0 & 0 & 0 & 0 & 0 & 0 & 0 & 0 & 0 & 0 & 0\end{array}$

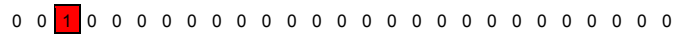

Top Left pixel of zero order

Top Center pixel of zero order 


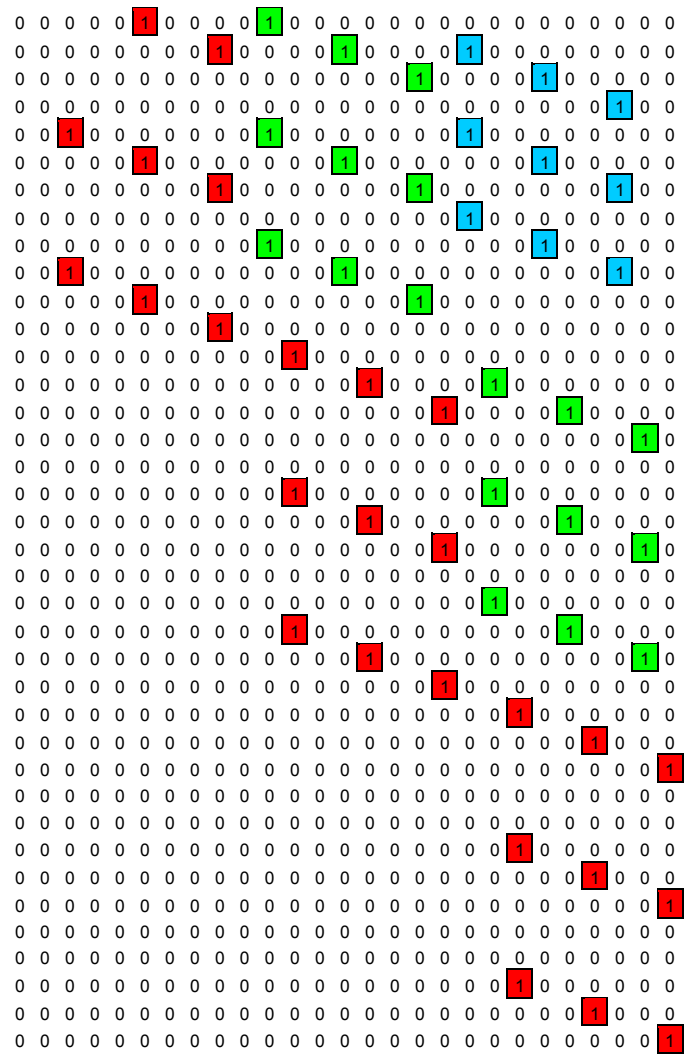

This example $\mathrm{H}$ matrix does illustrate that there is some type of a structure to the nonzero elements in the matrix, however, this particular representation may not necessarily be as insightful and may not provide any sort of mathematical processing advantages as other expressions of the system H matrix. Since each column of the CTIS H matrix represents a calibration image taken with the system, the specific order of the columns/calibration images within the matrix is completely arbitrary. Columns can be swapped and repositioned within the matrix at will. The same is also true for the rows of the $\mathrm{H}$ matrix. The user need only keep a consistent order when vectorizing the recorded CTIS image and the datacube (i.e. the first column of $\mathrm{H}$ matrix must correspond to the first element of the vectorized datacube, the first row of the $\mathrm{H}$ matrix corresponds to the first element in the vectorized CTIS image, etc.). Figure 6.9 on the following page presents a diagram showing two representations of the complete system $\mathrm{H}$ matrix for this example CTIS. The colored boxes correspond to the nonzero components in the matrix, where the color represents the wavelength band for each calibration image, while white boxes are used for components with a value of zero. The representation on the left is the same matrix as shown previously, while the representation on the right has rearranged the columns first based on the wavelength of the calibration image going from lowest to highest, and then subsequently by the spatial position of the point object in the zero order. It is important to note that both representations of the $\mathrm{H}$ matrix are equivalent, they are composed of the same information, only the order of elements in the $1 \mathrm{D}$ object vector describing the voxel irradiances are rearranged. This new representation of the $\mathrm{H}$ matrix has a clear diagonal like property to the non zero elements. 

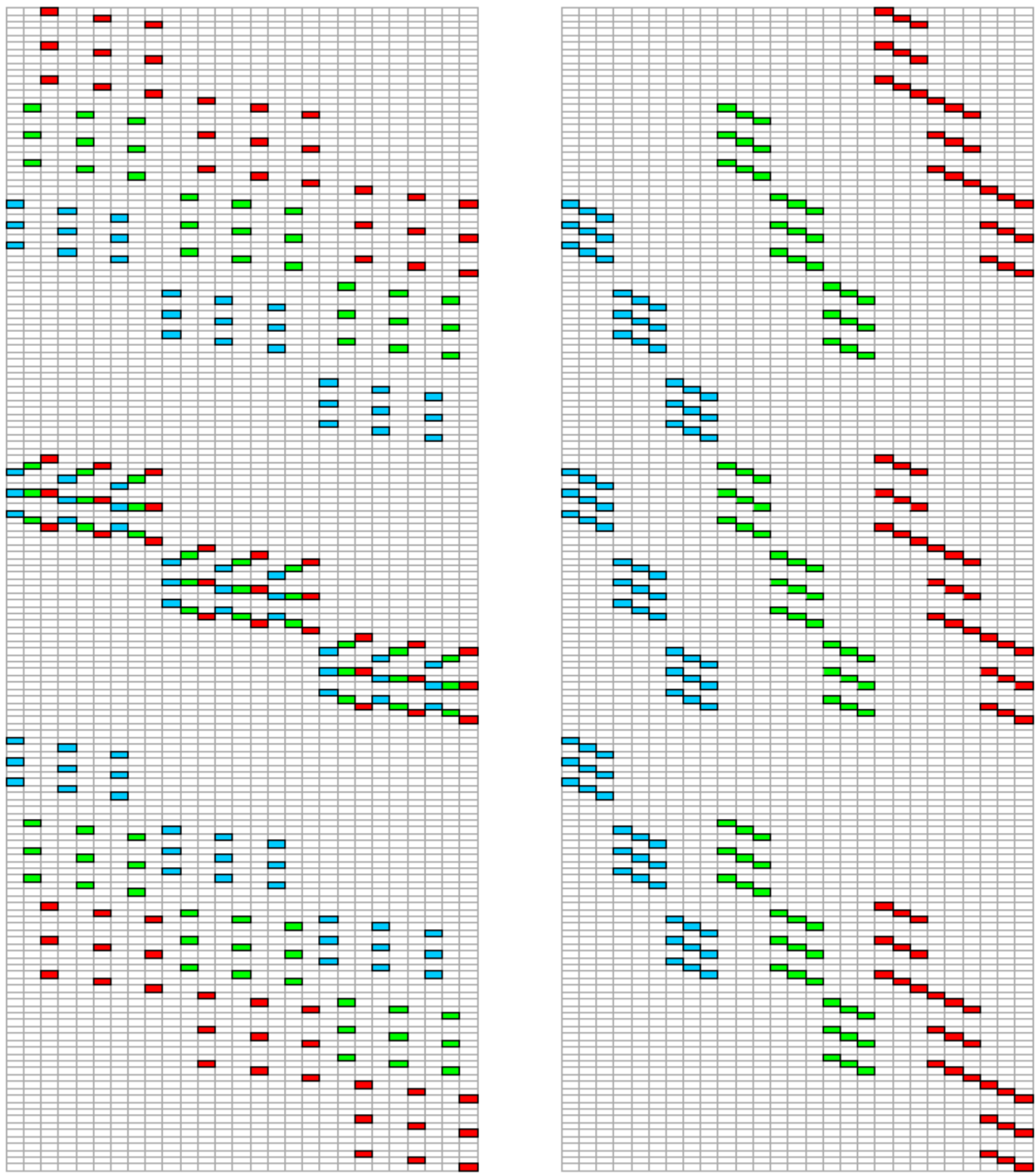

Figure 6.9: (left) Diagram of H matrix for example simple CTIS system. Order of calibration images/columns arranged first by spatial position of object in the zero order, and subsequently by wavelength going from lowest to highest. (right) Diagram of $\mathrm{H}$ matrix for the same example simple CTIS system with columns rearranged, first by wavelength and then by spatial position. This representation of the example CTIS H matrix can be described as being a Block Toeplitz matrix.

Specifically the H matrix for the CTIS system can be described as being a Sparse Block Toeplitz matrix. Before going on to describe why this particular CTIS H matrix has this property and how it can be applied to other CTIS systems, let us first review what exactly is a Toeplitz matrix. 


\subsection{Toeplitz Matrix Properties}

A Toeplitz matrix can be referred to as a diagonal constant matrix. It is a matrix where each descending diagonal element from left to right is the same. Some examples of a Toeplitz matrix are shown in figure 6.10 .

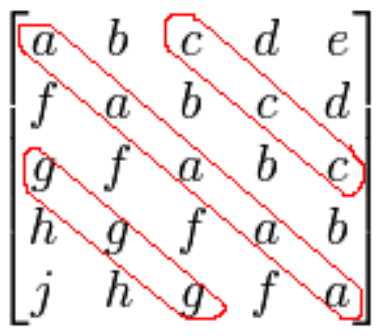

$$
A=\left[\begin{array}{cccccc}
a_{0} & a_{-1} & a_{-2} & \ldots & \ldots & a_{-n+1} \\
a_{1} & a_{0} & a_{-1} & \ddots & & \vdots \\
a_{2} & a_{1} & \ddots & \ddots & \ddots & \vdots \\
\vdots & \ddots & \ddots & \ddots & a_{-1} & a_{-2} \\
\vdots & & \ddots & a_{1} & a_{0} & a_{-1} \\
a_{n-1} & \ldots & \ldots & a_{2} & a_{1} & a_{0}
\end{array}\right]
$$

Figure 6.10: Examples of Toeplitz Matrices

Note that the values along all of the diagonals are the same. Another way to think of a Toeplitz matrix is that all of the columns contain the same elements in the same order, but are just shifted down by different amounts. For example the second column is the same as the first, but just vertically shifted down by one. The same pattern is true when comparing the second and third columns. Each subsequent column is the same as the previous but shifted down one, which ensures that all of the diagonal elements are constant.

\subsection{Toeplitz and Shift Invariance}

Using the new representation of the $\mathrm{H}$ matrix, it is clear that the entire matrix can be partitioned into sections where each matrix subsection has the properties of being a Toeplitz matrix. This means that the CTIS H matrix can be described as a block Toeplitz matrix.

The Toeplitz structure of the subsections is due to the shift invariant property of the CTIS system and the raster scanning used to vectorize images. The shift invariant property of the CTIS system allowed the calibration images for all the zero order spatial positions to be generated by a set of calibration images from a fixed spatial position. All of the generated images were just pixel shifted forms of the original images. Since the raster scanning used to vectorize the images goes from left to right across the pixel rows, when comparing the calibration images for a single wavelength point object at different spatial positions along a row, going from left to right, we find that the vectors contain the same data but are just shifted down by one for each subsequent spatial location along the row forming the Toeplitz structure. So provided that the assumption of shift invariance for the system is true, the complete system $\mathrm{H}$ matrix will be a block Toeplitz matrix with the number of blocks/Toeplitz subsections equal to the number of rows in the zero order times the number of wavelength bands in the datacube.

Note that this example is of a "simplified" CTIS system. While useful to see the general properties of the CTIS H matrix, the actual H matrices for constructed systems (such as the 
LWIR CTIS) will share the same general properties, but will be slightly more complicated. The $\mathrm{H}$ matrix for a constructed system will have orders of magnitude more elements, more lit pixels in each calibration image, and more shifted replications due to the higher spatial resolution. Visually interpreting sections of this matrix is far more challenging. As opposed to lighting only a single pixel, as in the example, the psf in each diffraction order will likely illuminate multiple pixels on separate rows causing the information from a specific order to appear in multiple sections of the column vector making identification of particular components a challenge. However, the system will still share the same block Toeplitz or shifted replication properties as the example.

\subsection{Sparse Property of the $\mathrm{H}$ matrix}

Another important property of the CTIS H matrix is the sparse nature of the matrix, meaning that a majority of the elements in the matrix will have a value of zero. This sparse property can readily be seen in the example system, where each calibration image has all zero value pixels with the exception of the one lit pixel in each diffraction order. Calibration images and system $\mathrm{H}$ matrices for constructed systems will share the same general sparse property but the amount of sparseness will depend upon the size of the point spread function in each diffraction order, noise levels in the recorded image, and whether a minimum pixel value threshold was applied to the calibration images in post processing. In practice it is best to adjust the camera's gain and offset settings to try and make the recorded background levels of the calibration images as close to zero as possible while still maintaining decent signal for the lit pixels across all of the calibration wavelength images while not saturating the pixels when recording CTIS scene images. Then by applying a low value threshold to the recorded calibration images the background pixels containing noise will now have values of zero producing a sparse system $\mathrm{H}$ matrix.

For these reasons the CTIS system H matrix can be described as a sparse block Toeplitz matrix. Having the sparse nature and shift invariant properties of the matrix provides a number of advantages when performing calculations and storing the $\mathrm{H}$ matrix. General techniques for sparse or Toeplitz matrices, as well as techniques specific to CTIS systems, can be applied which make the storage of extremely large $\mathrm{H}$ matrices as well as multiplications of the $\mathrm{H}$ matrix more practical on existing computer hardware. Some more details on these mathematical procedures will be covered in the following chapter on CTIS reconstructions. However, before moving on to the next chapter, there is one other important mathematical property of the example "simple" CTIS H matrix. The matrix is actually invertible.

\subsection{Peculiar Inverse Properties of the $\mathrm{H}$ matrix}

In comparison to the extremely large size $\mathrm{H}$ matrices for constructed CTIS systems, the $\mathrm{H}$ matrix for the "simplified" CTIS system presented in this chapter also allowed for the examination of other mathematical properties of the matrix, such as the rank of the matrix. The rank of a matrix describes how many of the matrix's columns or rows are linearly independent from each other. That is to say, it examines whether or not any of the rows or columns of the matrix could have the values that could be made by adding a linear combination of other rows or columns of the matrix. If a matrix has full rank, which by definition means that all of its columns are linearly independent, then some form of a matrix inverse can be calculated for that particular matrix. If 
the initial matrix is square, this means that the actual inverse can be determined. If the matrix is rectangular, then either the left or right inverse can be determined.

Students who have worked on CTIS in the past have stated that the H matrices for CTIS systems are thought to be singular, implying that an inverse does not exist. However, for the example system with $3 \times 3$ spatial and 3 spectral bands, the corresponding $\mathrm{H}$ matrix was found to be full rank. A direct left inverse of the matrix can be calculated, meaning that datacube reconstructions for the system could be directly calculated with a single matrix multiplication.

This has some important ramifications when it comes to performing spectral reconstructions with a CTIS system. In the past, when it was thought that the inverse to CTIS H matrices did not exist, even attempts at calculating the pseudoinverse of the matrix were not done due to the fact that the computation of a pseudoinverse on such a large matrix would be too computationally intensive. For this example system, not only can a pseudoinverse be calculated, the result would be an actual matrix inverse capable of providing a direct (non estimated) reconstructed datacube values.

Since it was found that the system matrix for this "simple" CTIS with its particular system parameters was invertible, a variety of CTIS systems, each with different parameters for the size of the zero order and the amount of dispersion in the diffraction orders, were also tested. The results from these tests are presented in the table below. Note that all of these CTIS system only have 9 diffraction orders, unlike the constructed LWIR CTIS which has 25 diffraction orders.

\section{Is a given 9 diffraction order CTIS H matrix invertible?}

\begin{tabular}{|c|c|c|c|c|c|c|c|c|c|c|c|c|c|c|c|c|}
\hline & \multicolumn{15}{|c|}{ Amount of Spectral Bands / Pixels of Dispersion } \\
\hline & & 1 & 2 & 3 & 4 & 5 & 6 & 7 & 8 & 9 & 10 & 11 & 12 & 13 & 14 & 15 \\
\hline \multirow{15}{*}{ 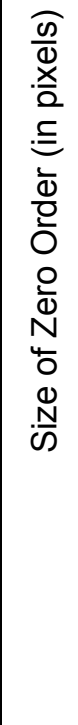 } & $1 \times 1$ & $\mathrm{Y}$ & $\mathrm{Y}$ & $\mathrm{Y}$ & $\mathrm{Y}$ & $\mathrm{Y}$ & $\mathrm{Y}$ & $\mathrm{Y}$ & $\mathrm{Y}$ & $\mathrm{Y}$ & $\mathrm{Y}$ & $\mathrm{Y}$ & $\mathrm{Y}$ & $\mathrm{Y}$ & $\mathrm{Y}$ & $\mathrm{Y}$ \\
\hline & $2 \times 2$ & $\mathrm{Y}$ & $\mathrm{Y}$ & $\mathrm{Y}$ & $\mathrm{Y}$ & $\mathrm{Y}$ & $\mathrm{Y}$ & $\mathrm{Y}$ & $\mathrm{Y}$ & $\mathrm{Y}$ & $\mathrm{Y}$ & $\mathrm{Y}$ & $\mathrm{Y}$ & $\mathrm{Y}$ & $\mathrm{Y}$ & $\mathrm{Y}$ \\
\hline & $3 \times 3$ & $Y$ & $\mathrm{Y}$ & $\mathrm{Y}$ & $Y$ & $\mathrm{Y}$ & $Y$ & $Y$ & $Y$ & $Y$ & $\mathrm{Y}$ & $\mathrm{Y}$ & $\mathrm{Y}$ & $\mathrm{Y}$ & $Y$ & $\mathrm{Y}$ \\
\hline & $4 \times 4$ & $Y$ & $Y$ & $Y$ & $Y$ & $Y$ & $Y$ & $Y$ & $Y$ & $Y$ & $Y$ & $Y$ & $Y$ & $Y$ & $Y$ & $Y$ \\
\hline & $5 \times 5$ & $Y$ & $Y$ & $Y$ & $Y$ & $Y$ & $Y$ & $Y$ & $Y$ & $Y$ & $Y$ & $Y$ & $Y$ & $Y$ & $Y$ & $\mathrm{Y}$ \\
\hline & $6 \times 6$ & $Y$ & $Y$ & $\mathrm{Y}$ & $\bar{Y}$ & $Y$ & $Y$ & $Y$ & $Y$ & $Y$ & $Y$ & $Y$ & $Y$ & $Y$ & $Y$ & $Y$ \\
\hline & $7 \times 7$ & $Y$ & $Y$ & $Y$ & $Y$ & $\mathrm{Y}$ & $Y$ & $Y$ & $Y$ & $Y$ & $\mathrm{~N}$ & $\mathrm{~N}$ & $\mathrm{~N}$ & $\mathrm{~N}$ & $\mathrm{~N}$ & $\mathrm{~N}$ \\
\hline & $8 \times 8$ & $Y$ & $Y$ & $Y$ & $Y$ & $Y$ & $Y$ & $Y$ & $Y$ & $Y$ & $\mathrm{~N}$ & $\mathrm{~N}$ & $\mathrm{~N}$ & $\mathrm{~N}$ & $\mathrm{~N}$ & $\mathrm{~N}$ \\
\hline & $9 \times 9$ & $Y$ & $Y$ & $Y$ & $Y$ & $Y$ & $Y$ & $Y$ & $Y$ & $Y$ & $\mathrm{~N}$ & $\mathrm{~N}$ & $\mathrm{~N}$ & $\mathrm{~N}$ & $\mathrm{~N}$ & $\mathrm{~N}$ \\
\hline & $10 \times 10$ & $Y$ & $Y$ & $Y$ & $Y$ & $Y$ & $Y$ & $Y$ & $Y$ & $Y$ & $\mathrm{~N}$ & $\mathrm{~N}$ & $\mathrm{~N}$ & $\mathrm{~N}$ & $\mathrm{~N}$ & $\mathrm{~N}$ \\
\hline & $11 \times 11$ & $Y$ & $Y$ & $Y$ & $Y$ & $Y$ & $Y$ & $Y$ & $Y$ & $Y$ & $\mathrm{~N}$ & $\mathrm{~N}$ & $\mathrm{~N}$ & $\mathrm{~N}$ & $\mathrm{~N}$ & $\mathrm{~N}$ \\
\hline & $12 \times 12$ & $Y$ & $Y$ & $Y$ & $Y$ & $Y$ & $Y$ & $Y$ & $Y$ & $Y$ & $\mathrm{~N}$ & $\mathrm{~N}$ & $\mathrm{~N}$ & $\mathrm{~N}$ & $\mathrm{~N}$ & $\mathrm{~N}$ \\
\hline & $13 \times 13$ & $Y$ & $Y$ & $Y$ & $Y$ & $Y$ & $Y$ & $Y$ & $Y$ & $Y$ & $\mathrm{~N}$ & $\mathrm{~N}$ & $\mathrm{~N}$ & $\mathrm{~N}$ & $\mathrm{~N}$ & $\mathrm{~N}$ \\
\hline & $14 \times 14$ & $Y$ & $Y$ & $Y$ & $Y$ & $Y$ & $Y$ & $Y$ & $Y$ & $Y$ & $\mathrm{~N}$ & $\mathrm{~N}$ & $\mathrm{~N}$ & $\mathrm{~N}$ & $\mathrm{~N}$ & $\mathrm{~N}$ \\
\hline & $15 \times 15$ & $Y$ & $Y$ & $Y$ & $Y$ & $Y$ & $Y$ & $Y$ & $Y$ & $Y$ & $\mathrm{~N}$ & $\mathrm{~N}$ & $N$ & $\mathrm{~N}$ & $N$ & $\mathrm{~N}$ \\
\hline
\end{tabular}

Table 2: Evaluating existence of $\mathrm{H}$ matrix left inverse for various $3 \times 3$ CTIS designs 
The results showed that for any 9 diffraction order CTIS with a zero order of size $6 \times 6$ pixels or smaller, the corresponding $\mathrm{H}$ matrix would be invertible no matter how many spectral bands the system had. It also showed that if the CTIS had 9 spectral bands or less, no matter how large the zero order was, the $\mathrm{H}$ matrix would be invertible. This pattern continued out to as far as the memory limitations of testing computer would allow. For example, a system with a $4 \times 4$ zero order and 70 spectral bands was invertible, as was a $6 \times 6$ spatial resolution system with 50 spectral bands.

\subsection{Expanding the range of invertible CTIS systems}

These results were quite interesting, especially the sharp cutoff between invertible and noninvertible systems when the zero order is larger than 6x6 and the dispersion is larger than 9 pixels. This cutoff appears to be a property of the inherent nature of the multiplexing in the CTIS system, which depends on the zero order size, amount of spectral dispersion, number of diffraction orders, and other parameters of the system.

As previously noted, these results were for CTIS systems with 9 diffraction orders producing a $3 \times 3$ diffraction pattern. A second set of tests were run on CTIS systems that contained only 5 diffraction orders as opposed to 9 . The location pattern for these 5 orders were the same as the $3 \times 3$ pattern, but with the 4 diagonal orders removed. In this case, the cutoff between invertible and non-invertible was shifted to systems with lower spatial and spectral resolutions. Fewer systems were invertible in comparison to CTIS systems with 9 diffraction orders. It appears as though the added diffraction orders provide more unique multiplexed information in the $\mathrm{H}$ matrix allowing more systems to be invertible.

The next step was to test CTIS systems with 25 diffraction orders ( $5 \times 5$ pattern). In addition to just having more orders, these tests differed than the previous tests in the fact that the discrete to discrete mapping of object voxels to image pixels no longer had perfect pixel registration in all of the orders. That is to say that within a given diffraction order, light from a single discrete voxel may now be contributing signal to multiple pixels on the focal plane array instead of a single pixel as before. This is because in the off diagonal diffraction orders of the outermost ring, and in the inner 9 orders, the location of the mapping of a voxel doesn't necessarily correspond to the center of a pixel. The computer simulation used to generate calibration images for the test systems accounted for this by interpolating the signal from each voxel onto the focal plane depending upon its mapping locations.

The results for the $5 \times 5$ pattern testing, given below, show that all of the CTIS systems that were tested before running into computer memory limitations are invertible. Systems with zero order and spectral parameters that were previously not invertible using a $3 \times 3$ diffraction order pattern are now shown to be invertible with a $5 \times 5$ diffraction pattern. 
Is a given $\mathbf{2 5}$ diffraction order CTIS $\mathrm{H}$ matrix invertible?

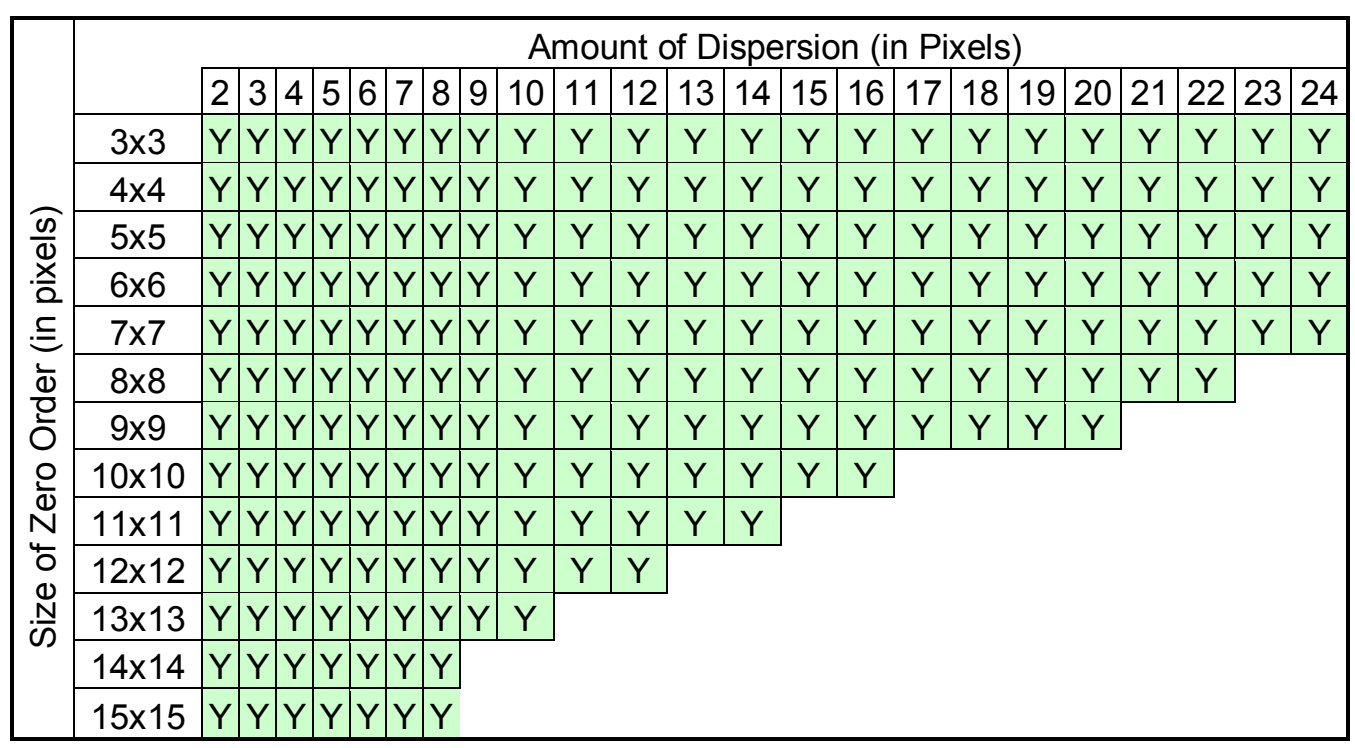

Table 3: Evaluating existence of $\mathrm{H}$ matrix left inverse for various $5 \times 5$ CTIS designs

These results for the $5 \times 5$ pattern testing seem to indicate that a direct left inverse of the LWIR CTIS H matrix may exist. Recall that the LWIR CTIS datacube has a zero order size that is 33 pixels by 33 pixels with 70 pixels of dispersion. Moving from a $3 \times 3$ dispersion pattern to a $5 \times 5$ dispersion pattern has clearly expanded the range of invertible CTIS H matrices. Intuitively this makes sense because, with a fixed amount of dispersion, the $5 \times 5$ pattern will contain all of the information from a corresponding $3 \times 3$ pattern in addition to some new information from the added diffraction orders at new dispersion angles. The more diffraction orders you have at unique dispersion angles, the more unique information you have recorded in your image. These added orders at new angles give us more information, or a better understanding, of the spectral signature multiplexing of the various spatial locations. While the switch from a $3 \times 3$ pattern to a $5 \times 5$ expanded the range of invertible CTIS H matrices, there may still be some cutoff for noninvertible $5 \times 5$ systems, just as there was in the $3 \times 3$ case, outside the tested values. Due to computer memory limitations in the testing, we were unable to find or determine if such a threshold exists.

If the $\mathrm{H}$ matrix for the $5 \times 5$ LWIR CTIS system did have a direct left inverse, what benefit would it be? One clear benefit is that reconstructions for the system could be carried out in just a single mathematical step, a single matrix-vector multiplication. However, recall that the LWIR CTIS H matrix (and possible left inverse) would have over 23 billion elements. The reconstructions would only require one step, but a lot of computing power would be needed to complete that single action. There is no guarantee that the left inverse would be any faster than the standard reconstruction techniques. Not to mention the fact that the computing power problem only gets compounded when using higher resolution sensors, like those found in visible systems. The following chapter will evaluate this and other CTIS reconstructions in an attempt to find methods that can dramatically increase the speed of CTIS reconstructions. 


\section{CHAPTER 7: DESCRIPTION AND COMPARISON OF CTIS RECONSTRUCTION ALGORITHMS}

\subsection{Overview}

Once a CTIS image for a particular scene has been recorded, we are left with the task of using that single image to determine all of spatial and spectral irradiance properties of the scene. Determining the datacube irradiance values from the recorded CTIS image will require significantly more work than what is needed in conventional scanning imaging spectrometers. In a conventional scanning system you can often directly relate irradiance values in the datacube to the signal from an individual pixel. However, due to the multiplexing of the spatial and spectral data in the CTIS image, there is not a simple one to one direct relationship for all of the datacube irradiance values. A given pixel on the focal plane will receive light from a variety of points along a diagonal line through the datacube with some pixels on the focal plane having more multiplexing than others, adding complexity to our ability to reconstruct the datacube irradiance values based on the raw image data.

Our analysis of the datacube reconstruction process will be based upon the standard imaging equation:

$$
\mathrm{g}=\mathrm{Hf}
$$

With this equation, we ultimately want to use our existing knowledge of the optical system and its calibrated properties, $\mathrm{H}$, to take the given image information, $\mathrm{g}$, and find the irradiance values of the datacube, $f$, that created the image.

There are a number of ways to approach this reconstruction problem. First, you need to clearly define how you are going to describe the datacube and all of the other elements of the imaging equation. Historically, CTIS systems have used an approach where the datacube and image are represented by discrete vectors, with the image vector being composed of discrete pixel values, the datacube vector being composed of discrete irradiance values corresponding to the irradiance of a small volume element, or voxel, in the three dimensional datacube, and the optical system is described by a rectangular system matrix $\mathrm{H}$. In this description the imaging equation is given by a matrix vector multiplication, where the multiplication of the $\mathrm{H}$ matrix times the datacube vector provides the mapping of the given datacube voxels to a pixel image. This is the description which will be used in the work for this dissertation, but recognize that there are other ways to represent the system. For example, Jim Scholl recently completed some reconstruction work where the datacube is described as being composed of a combination of continuous three dimensional frequencies, similar to a Fourier series, spanning the three dimensional datacube space rather than using the voxel approach. ${ }^{20}$

Once you have chosen how you are going to describe the imaging equation parameters, there is still the problem of how to actually calculate the irradiance values describing the datacube. Once again, there are a number of different mathematical ways to try and determine, or at least estimate, the actual datacube values corresponding to a particular CTIS image. 
In this investigation of datacube reconstruction algorithms, we will cover reconstructions using the inverse of $\mathrm{H}$, the pseudo inverse of $\mathrm{H}$, and the conventional iterative reconstruction techniques EM and MART. Note that the list of possible reconstruction techniques is not limited to what is presented here. Nathan Hagen has provided information on a variety of other techniques in his dissertation as well. ${ }^{21}$ Following this description, we will then evaluate the speed of each reconstruction method in order to compare the relative speed of CTIS reconstructions using each technique. This speed comparison will be evaluated for both sequential and parallel processing of the data using various techniques. Ultimately, the information from these comparisons can be used along with the relative performance/quality of reconstructions to try and optimize the reconstruction process to achieve reconstructions of acceptable quality in the shortest amount of time possible.

\subsection{Reconstruction by Finding an Inverse of the $\mathrm{H}$ matrix}

By just looking at the imaging equation and considering the matrix/vector representation of its parameters, it is clear that something like the inverse of the $\mathrm{H}$ matrix could be used in order to find the vector $\mathrm{f}$, describing the datacube.

If the inverse of $\mathrm{H}$ existed you could do the following.

$\mathrm{g}=\mathrm{H} \mathrm{f}$

$\mathrm{H}^{-1} \mathrm{~g}=\mathrm{H}^{-1} \mathrm{H} \mathrm{f}$

$\mathrm{H}^{-1} \mathrm{~g}=\mathrm{If}$

$\mathrm{H}^{-1} \mathrm{~g}=\mathrm{f}$

(I is the identity matrix)

You would now have an expression for the datacube, $f$, that produced the recorded image, $g$, and you would be done.

Unfortunately the strict definition of a matrix inverse is only defined for square matrices. Since the dimensions of the CTIS H matrix has the number of rows equaling the number of pixels in the focal plane and the number of columns equaling the number of voxels in the datacube, the CTIS H matrix is almost guaranteed to be a rectangular matrix, which by definition will not have a strict matrix inverse.

However, in the previous chapter we discovered that for certain CTIS systems a left inverse of the $\mathrm{H}$ matrix does exist, but this is by no means true for all CTIS systems. For systems that have a left inverse of the $\mathrm{H}$ matrix, the reconstruction of the datacube can be done in a single mathematical step. This approach is referred to as the direct left inverse technique and is accomplished by merely multiplying the left inverse of the system $\mathrm{H}$ matrix to the recorded CTIS image vector, $g$, as shown in the following equations:

$\mathrm{g}=\mathrm{H} \mathrm{f}$

$\mathrm{H}^{-1}{ }_{\text {left }} \mathrm{g}=\left(\mathrm{H}^{-1}\right.$ left $\left.\mathrm{H}\right) \mathrm{f}$

$\mathrm{H}^{-1}{ }_{\text {left }} \mathrm{g}=(\mathrm{I}) \mathrm{f}$

$\mathrm{H}^{-1}{ }_{\text {left }} \mathrm{g}=\mathrm{f}$ 
For systems where the left inverse of the system matrix does not exist, it would still be possible to calculate a reconstructed datacube using the pseudoinverse of the $\mathrm{H}$ matrix, $\mathrm{H}^{+}$. This approach will also consist of just a single matrix multiplication.

$\mathrm{H}^{+} \mathrm{g}=\mathrm{f}$

In many CTIS systems, especially those with larger datacubes, the shear size of the system $\mathrm{H}$ matrix will make it impractical to even calculate either the left inverse of $\mathrm{H}$ or the pseudoinverse of H. However, these approaches may be useful for simpler/lower resolution CTIS systems that do not have such issues with computation. In the future, as more computing resources become available, these approaches may become more practical for use in higher resolution systems, but in the mean time other approaches can be considered or CTIS systems can be specifically tailored to use these reconstruction techniques.

Historically the computational challenges of these methods have been overcome by using iterative reconstruction approaches, which only need to perform operations using the extremely sparse, and more computationally friendly, $\mathrm{H}$ matrix itself as opposed to the inverse of $\mathrm{H}$ or the pseudoinverse of $\mathrm{H}$.

\subsection{Conventional Iterative Reconstruction Techniques EM and MART}

The two conventional iterative reconstruction methods used in CTIS systems are Expectation Maximization (EM) and Multiplicative Algebraic Reconstruction Technique (MART). The mathematical expressions for these iterative reconstructions techniques are as follows:

$$
\begin{array}{ll}
\text { EM: } & \hat{f}^{(k+1)}=\frac{\hat{f}^{(k)}}{\sum_{m=1}^{M} H_{m n}} H^{T}\left(\frac{g}{H \hat{f}^{(k)}}\right) \\
\text { MART: } & \hat{f}^{(k+1)}=\hat{f}^{(k)}\left(\frac{H^{T} g}{H^{T} H \hat{f}^{(k)}}\right)
\end{array}
$$

Upon first glance, it is difficult to understand, or even just get a feel for what each of these iterative reconstruction techniques are doing, especially if you do not have an extensive mathematical background. At a minimum, the optical engineer of the system must understand that these mathematical operations are iterating on a guess/estimate of the datacube, $\hat{f}$, using the recorded focal plane data, $\mathrm{g}$, and system information, $\mathrm{H}$, to try and develop a better estimate of the spectral properties of each spatial location of the scene, thus performing the mathematical reconstruction of the data cube.

The optical engineer can get a better understanding of how each of these techniques works, as well as determining some of the properties and differences between the two techniques, by examining each component of these equations and relating it to the standard imaging equation. Each component or step can be related to how the various spatial/spectral properties of the 
collected light form the pixel values recorded by the focal plane. In the following pages, a walkthrough of the various required steps for each iterative method are presented along with a written description and illustration of what each step is doing and what that step is trying to accomplish. Our analysis begins with a look at Expectation Maximization and will be followed by a description of how the Multiplicative Algebraic Reconstruction Technique works.

\subsection{Description of How Expectation Maximization (EM) Works}

$$
\text { EM: } \quad \hat{f}^{(k+1)}=\frac{\hat{f}^{(k)}}{\sum_{m=1}^{M} H_{m n}} H^{T}\left(\frac{g}{H \hat{f}^{(k)}}\right)
$$

Step 1) Start with an initial random guess of what the answer might be. For CTIS we will generate an initial data cube, $\hat{f}^{(0)}$, that is filled with random irradiance values. See figure 7.1 .

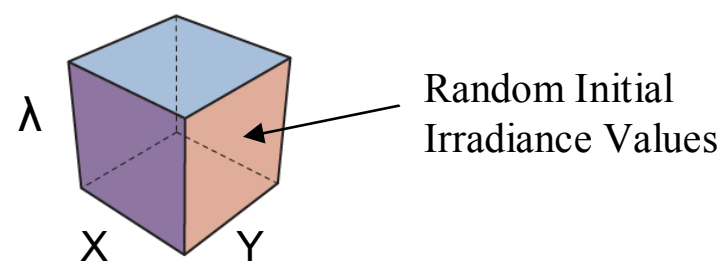

Figure 7.1: Initial random estimate of datacube

Step 2) How close was your guess? If the CTIS system were to actually view a scene whose spatial/spectral properties were described by your randomly generated datacube, would the image recorded by the CTIS viewing that scene be similar to the measured CTIS image, g, we are trying to reconstruct? That similarity can be determined by calculating the ratio of the pixel values in the measured image we are trying to reconstruct to the pixel values of the image corresponding to the current estimate.

First, calculate $g_{\text {estimated }}=H \hat{f}^{(0)}$ to determine what the resulting image would be for our current estimate of the datacube. Just as in the standard imaging equation, this calculation projects the initial random datacube, $\hat{f}^{(0)}$, to a focal plane image, $\mathrm{g}_{\text {estimated, }}$ as illustrated in figure 7.2. We say the datacube is projected from datacube space to an image in image space. 


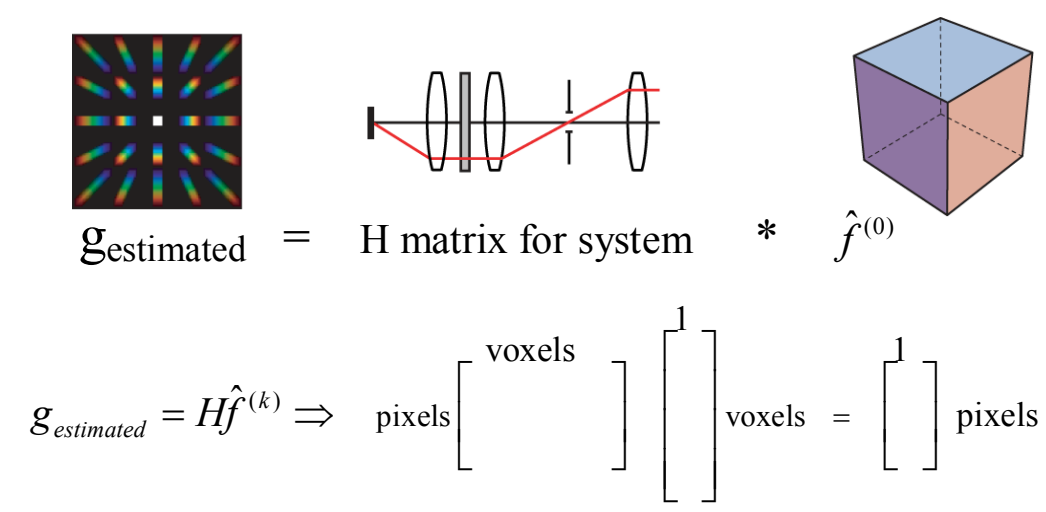

Figure 7.2: Projection of datacube estimate to image space

Step 3) We then divide element by element, the actual measured pixel values from the image we are trying to reconstruct, $g$, by the current estimated image we just calculated, gestimated. This resulting vector, $\left(g / H \hat{f}^{(0)}\right)$, is a list of factors of how similar the pixel values in the images are. If the images are similar, many of these values will be close to 1 . Those elements that are not similar will have a value far from 1 and will later result in changes to our estimate. Specifically, the factor for dissimilar pixels will be either greater than 1 or a value less than one but greater than zero depending upon the actual and estimated pixel values. See figure 7.3.

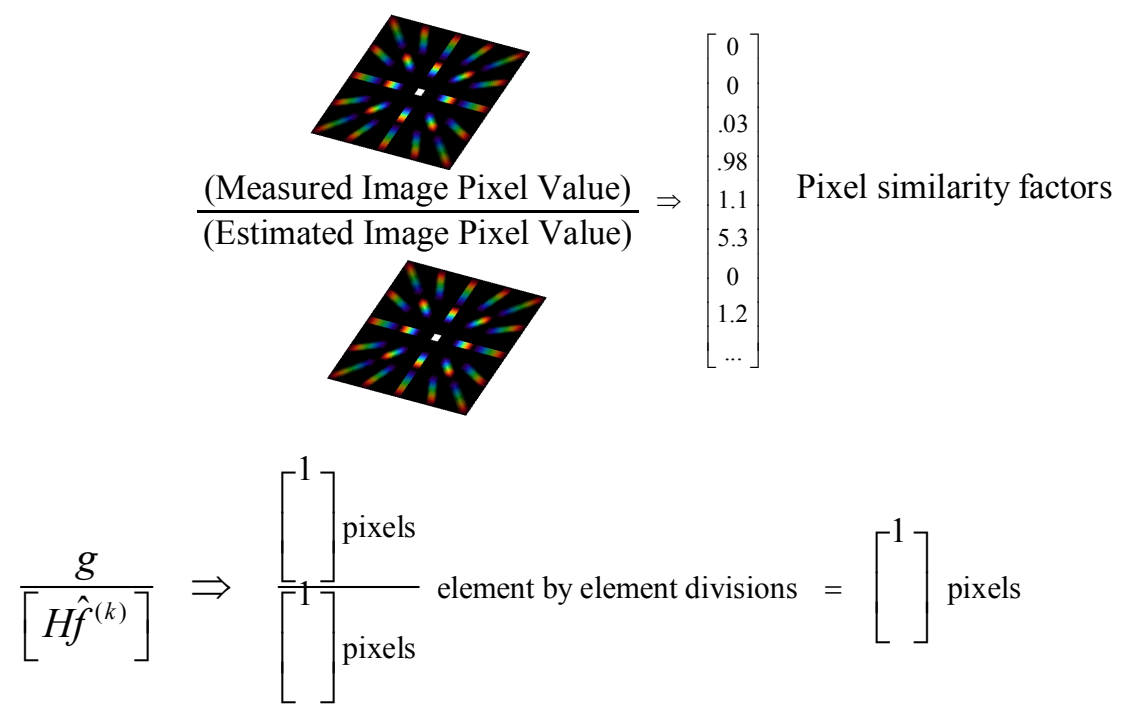

Figure 7.3: Calculating similarity between measured and estimated image.

Note on the implementation of this procedure, if there are any pixels in the estimated image that have a value of zero, for such pixels we instead divide by 1 when calculating the vector of similarity factors to avoid a division by zero error. Also note that any pixel in the measured image, $g$, which has a value of 0 will always result in a corresponding value of 0 in the vector of similarity factors. 
Step 3) In the next steps, we effectively want to relate the calculated similarities between the two images to similarities/dissimilarities between the actual datacube and our current estimate of what that datacube might be. By knowing how close our estimate of the datacube is to the actual datacube, we can form an update to our estimate in an attempt to get closer to the actual values.

To do this, we first calculate $\mathrm{H}$ transpose times the vector of pixel similarity factors. By using $\mathrm{H}$ transpose, we back project the image similarity factors back into datacube space. Another way to see this is to consider the following. Recall that each column of the $\mathrm{H}$ matrix corresponds to a calibration image. This calibration image is the actual recorded CTIS image when viewing a scene where only a voxel/single monochromatic point of the datacube is lit. For the $\mathrm{H}$ transpose matrix, the dimensions are flipped. Now, each row in $\mathrm{H}$ transpose corresponds to a calibration image. When calculating $H^{T}\left(g / H \hat{f}^{(0)}\right)$, each element of the resulting vector will be like the dot product of a calibration image and the similarity vector, as shown in figure 7.4.

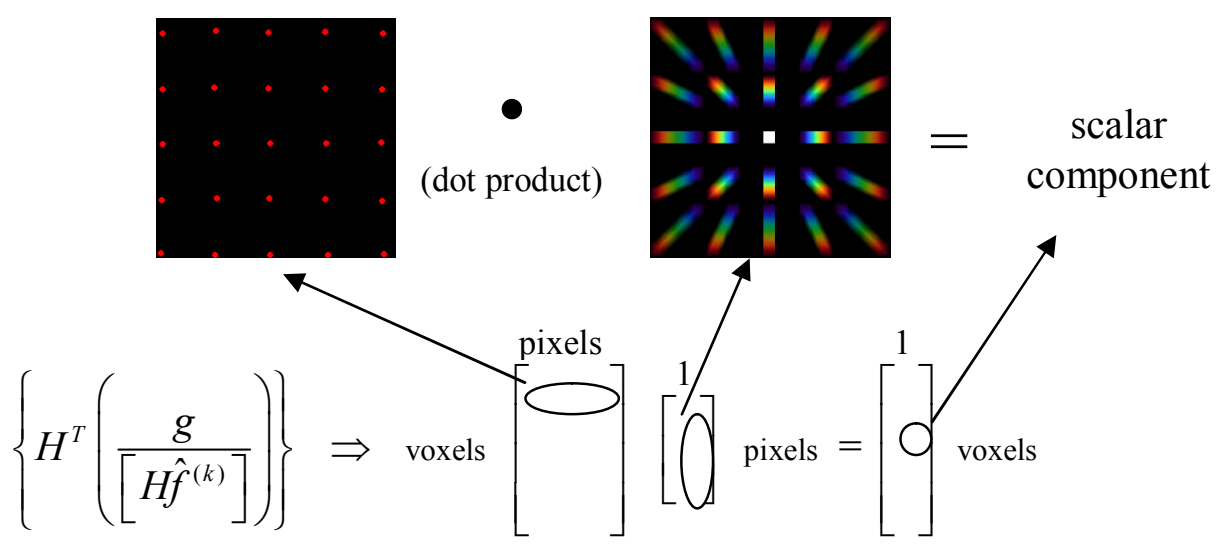

Figure 7.4: Illustration of how the scalar elements of the resulting back projected vector are given by the dot product of a calibration image and a CTIS image.

Consider the case of just multiplying $\mathrm{H}^{\mathrm{T}}$ by a regular CTIS image $\mathrm{g}$. When you take the dot product of a calibration image and the CTIS image, the resulting scalar component is given by the sum of the products of the shared pixels between the calibration image and the CTIS image. If the lit pixels on the image correspond well to the lit pixels of the calibration image for a particular voxel, the resulting scalar value for that voxel will be quite large. This provides a method of determining how much contribution a given voxel might have towards the generation of the CTIS image. If the calibration image for a voxel did not share any lit pixels with the CTIS image, then the resulting scalar, and that voxel's contribution to the image would be zero. It is this relation that allows $\mathrm{H}$ transpose times an image to correspond to backprojecting that image into datacube space. 
(Note that in step one it can be better to use $\mathrm{h}$ transpose times the recorded image $\mathrm{g}$ for your initial datacube guess instead of using random values.)

In the case of multiplying $\mathrm{H}^{\mathrm{T}}$ by the vector of pixel similarity factors, the result will be a single vector which is related to the similarity between the actual datacube and our current estimate of the datacube. It will eventually allow us to see if our initial estimate of the voxel value is too high or too low from the actual voxel values.

Step 4) Consider the case where the similarity vector has values of 1 at all the lit pixel locations of the calibration image. In this case the pixel values from the measured CTIS image exactly equal the pixel values from our currently estimated datacube image at all the nonzero pixel locations of the calibration image. The resulting component value of $H^{T}\left(g / H \hat{f}^{(0)}\right)$ would equal the total sum of the products

between all the pixel values in the calibration image times the corresponding values in the similarity vector, which are all one in this example. For this case the resulting component is equal to the total sum of all the pixel values in the calibration image. This is why in this step we divide, element by element, the values in this resulting vector by the corresponding total signal in each calibration image. Effectively, we are trying to normalize these similarity values by the total irradiance recorded in each calibration image. $\sum_{m=1}^{M} H_{m n}$ is a vector whose values are given by the sum of each column of $\mathrm{H}$. In other words, the values of $\sum_{m=1}^{M} H_{m n}$ are equal to the total signal in each system calibration image. So if the similarity vector had values of 1 at all the lit pixel locations of the calibration image, then after the normalization by calibration image irradiances, the resulting element would have a value of 1 . Ultimately, the values in this resulting vector, after the normalization, will serve as an update factor to our estimate of the datacube, as illustrated in figure 7.5.

$\frac{H^{T}\left(\frac{g}{H \hat{f}^{(k)}}\right)}{\sum_{m=1}^{M} H_{m n}}$

is a vector of update factors to our initial estimate of the datacube.

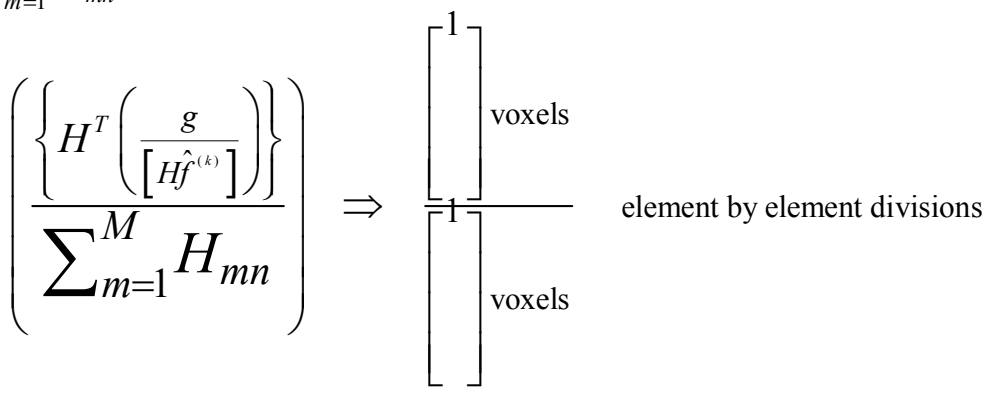




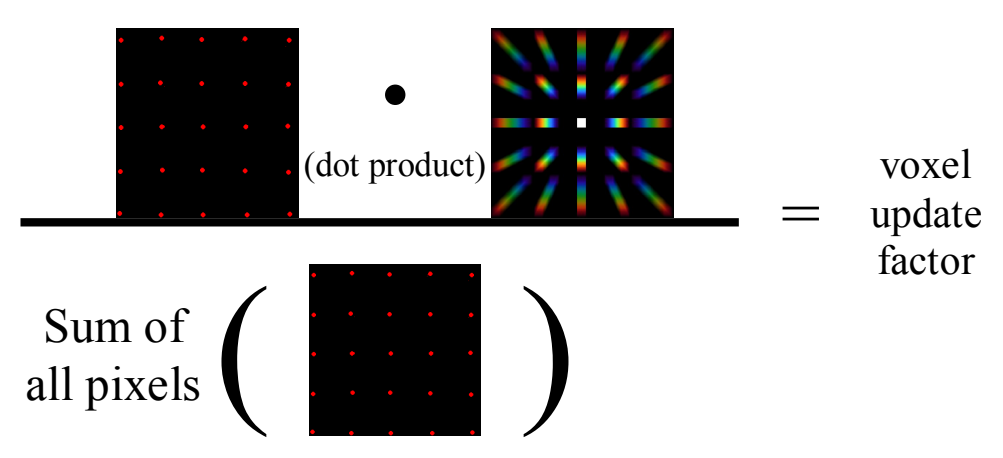

Figure 7.5: Illustration of how voxel update factors are calculated using calibration images

If update factor $>1$, the initial estimate for that voxel is too low.

If update factor $<1$, the estimate for that voxel is too high.

If update factor $=1$, no change is needed for our estimate of that voxel.

If update factor $=0$, that voxel is not present in the recorded image.

Another more realistic scenario, which illustrates how this step works, is to consider the case where the values of the similarity vector are all low, less than one. This implies that the pixel values for our current estimate of the datacube are significantly higher than the recorded image. When the dot product between a calibration image and the similarity vector is calculated, the result would be something like the following:

$$
\frac{V_{1}(.01)+V_{2}(.1)+V_{3}(.12)+\ldots}{V_{1}+V_{2}+V_{3}+\ldots}=\text { scalar value }<1
$$

Since the components of the similarity vector are all less than 1 , the corresponding normalized value of the update vector component will also be less than one. This means our initial estimate of the datacube irradiance value is too high, which is why the pixels of the CTIS image corresponding to our estimate were much higher than the actual recorded image. When we multiply this update factor by our original voxel irradiance estimate, it will decrease our estimated irradiance value for that voxel.

Step 5) In this final step, we create a new estimate of the datacube by multiplying our original estimate of the datacube by the vector of scaling voxel update factors. See figure 7.6. We are in effect trying to perform an educated rescaling of our initial guess in an attempt to get closer to the actual values. 


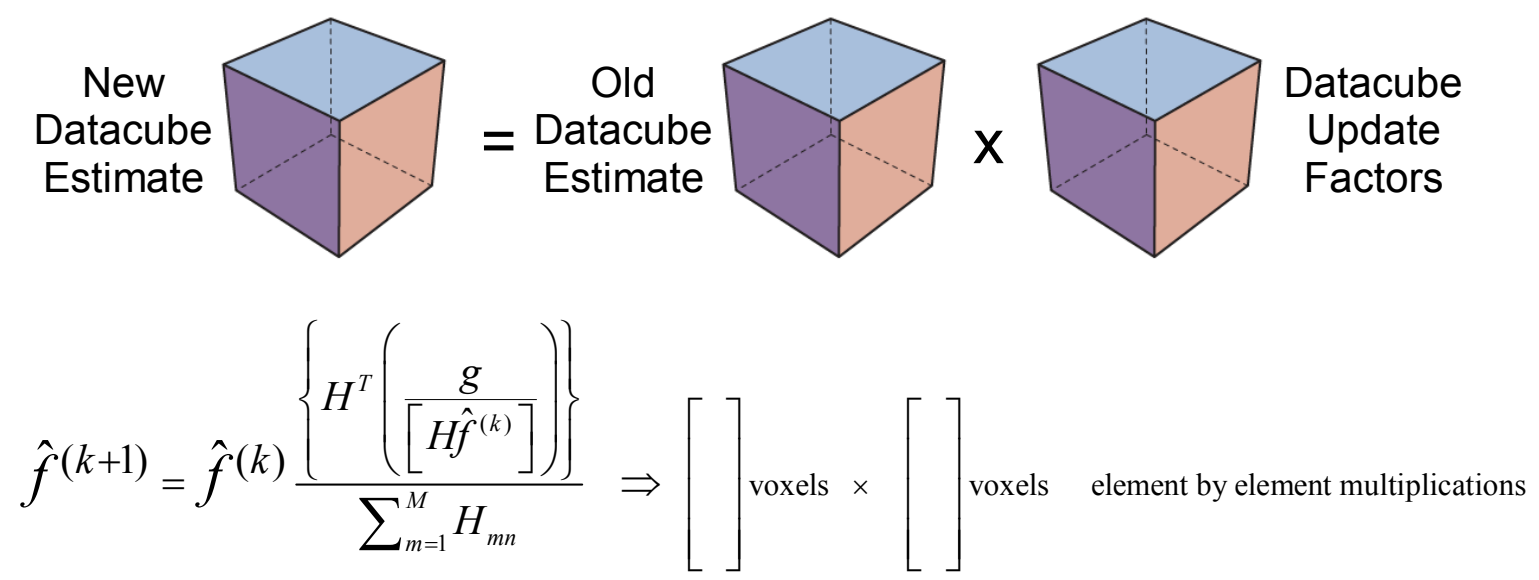

Figure 7.6: Illustration of how original estimate of the datacube is scaled by updating factors to create a new estimate of the datacube.

This completes one iteration of the Expectation Maximization reconstruction method. If needed, the result from step 5 can be used as the input for a subsequent iteration repeating the process from steps 2 through 5 again.

When using this method the largest changes to the estimated datacube will be made in just the first few iterations, which is the case for the MART technique as well. Subsequent iterations will produce smaller changes trying to fine tune the estimate to the actual datacube values. It is also important to note that as long as your initial estimate of the datacube does not contain any negative values, each subsequent estimate produced by EM will also only contain positive or zero values. This can be useful for the CTIS system, especially when viewing scenes where many voxel values are close to zero, since the irradiance values in the estimated datacube will never be negative. This positivity constraint is not present in the direct left inverse technique.

A similar analysis, albeit slightly more condensed, of the multiplicative algebraic reconstruction technique (MART) is presented on the following page, helping to illustrate what each process is doing and how the overall process differs from Expectation Maximization (EM). To briefly summarize, in contrast to EM, where the similarity/update factors were initially calculated in image space, MART will do something similar, but in datacube space. We will also find that the updates to the estimated datacube are "smoother" for MART than EM. This is primarily due to the fact that MART introduces more blurring operations to the data then EM since it uses more $\mathrm{H}$ matrix operations than EM. A description of this blurring is also found in the following MART description. 


\subsection{Description of How Multiplicative Algebraic Reconstruction Technique Works}

$$
\text { MART: } \quad \hat{f}^{(k+1)}=\hat{f}^{(k)}\left(\frac{H^{T} g}{H^{T} H \hat{f}^{(k)}}\right)
$$

Step 1) Generate an initial estimate of the datacube irradiances vector, $\hat{f}^{(0)}$.

Step 2) Calculate $H^{T}$.

In this step the CTIS image we are trying to reconstruct, $g$, is backprojected into datacube space. As previously described in the EM example, multiplying $\mathrm{H}$ transpose times an image provides a method of determining how much contribution a given voxel might have towards the generation of that CTIS image. Note that this calculation only needs to be done once. Any subsequent iterations will not require this step. You can just use the previously calculated values.

In contrast to EM, where the similarity/update factors were initially calculated in image space, MART will do something similar, but in datacube space. We will use this information from the back projected CTIS image to eventually form an update of our initial estimate.

Step 3) Calculate $H \hat{f}^{(0)}$.

Project the initial datacube estimate to image space and determine the resulting CTIS image from that particular datacube.

Step 4) Calculate $H^{T}\left(H \hat{f}^{(0)}\right)$.

Backproject the image you just calculated from the datacube estimate back into datacube space. This step may seem a bit strange, but it important to note that the resulting datacube will most likely not be the same datacube estimate you began with. Only certain types of matrices have the property of $\mathrm{H}^{\mathrm{T}} \mathrm{H}$ equaling the identity matrix. For other matrices, the operations involving $\mathrm{H}_{\text {or }} \mathrm{H}^{\mathrm{T}}$ will produce a certain amount of blurring in the result. So, $\mathrm{Hf}^{(\mathrm{k})}$ is actually a "blurred" estimate of the CTIS image for that datacube. $\mathrm{H}^{\mathrm{T}} \mathrm{H} \mathrm{f}^{(\mathrm{k})}$, being a forward and then back projection of the datacube estimate, will have two associated blurrings to the data.

Step 5) Calculate $\left(\frac{H^{T} g}{H^{T} H \hat{f}^{(0)}}\right)$.

This is just an element by element division of the components of the two datacube vectors, providing similarity factors between the back projected raw CTIS image and the forward and back projected datacube estimate. The result will form the scaling update vector to the initial datacube estimate.

Step 6) $\quad$ Calculate $\hat{f}^{(0)}\left(\frac{H^{T} g}{H^{T} H \hat{f}^{(0)}}\right)$.

Multiply the initial datacube estimate by the scaling update factors. If needed use this newly calculated estimate as the input for another iteration of steps 3-6. 


\subsection{Speed of Reconstruction Considerations}

Before continuing on with the discussions of reconstructions in the CTIS system and the time needed to perform the reconstructions, let us temporarily put aside our knowledge of the optical system, and focus on a particular mathematical property that will be useful in our system evaluation later on.

Consider the general case of multiplying a rectangular $\mathrm{m} \times \mathrm{n}$ matrix, $\mathrm{A}$, (containing $\mathrm{m}$ rows and $\mathrm{n}$ columns) by a column vector, $\mathrm{B}$, with dimensions $\mathrm{n} \times 1$. How many calculations are needed to complete this matrix vector multiplication operation?

To evaluate the number of calculations needed, let us look at the example of multiplying a $4 \times 3$ matrix by a $3 \times 1$ vector as a useful illustration.

$$
\begin{gathered}
\text { (n columns, } \mathrm{n}=3) \\
(\mathrm{m} \text { rows, } \mathrm{m}=4)\left[\begin{array}{lll}
a_{11} & a_{12} & a_{13} \\
a_{21} & a_{22} & a_{23} \\
a_{31} & a_{32} & a_{33} \\
a_{41} & a_{42} & a_{43}
\end{array}\right] \text { (n=3) }\left[\begin{array}{l}
b_{1} \\
b_{2} \\
b_{3}
\end{array}\right]=\left[\begin{array}{l}
\left(a_{11} b_{1}+a_{12} b_{2}+a_{13} b_{3}\right) \\
\left(a_{21} b_{1}+a_{22} b_{2}+a_{23} b_{3}\right) \\
\left(a_{31} b_{1}+a_{32} b_{2}+a_{33} b_{3}\right) \\
\left(a_{41} b_{1}+a_{42} b_{2}+a_{43} b_{3}\right)
\end{array}\right]
\end{gathered}
$$

In this example, the first component of the resulting vector is given by the value $\left(a_{11} b_{1}+a_{12} b_{2}+\right.$ $a_{13} b_{3}$ ). To find this component $n$ multiplications are required and (n-1) additions are required. So for the example case, $n=3$, we see that 3 multiplications are performed $\left(a_{11} b_{1}, a_{12} b_{2}, a_{13} b_{3}\right)$ and 2 additions are performed to determine the value of the component, $\left(a_{11} b_{1}+a_{12} b_{2}+a_{13} b_{3}\right)$. Each subsequent component value in vector will require the same $n+(n-1)$, or equivalently $(2 n-1)$, calculations needed to determine the component value. Since there will be $\mathrm{m}$ components in the resulting vector, the total number of calculations needed to perform the matrix vector multiplication is $(2 \mathrm{n}-1) \mathrm{m}$, or equivalently $2 \mathrm{~nm}-\mathrm{m}$.

If the user knows the matrix A ahead of time, there may be some benefit to the number of calculations needed to perform the matrix multiplication. Consider the following case, where a number of the components in the matrix A have a value of 0 .

$$
\begin{gathered}
(\mathrm{n} \text { columns, } \mathrm{n}=3) \\
(\mathrm{m} \text { rows, } \mathrm{m}=4)\left[\begin{array}{ccc}
a_{11} & a_{12} & 0 \\
0 & 0 & a_{23} \\
a_{31} & 0 & a_{33} \\
0 & a_{42} & 0
\end{array}\right] \quad(\mathrm{n}=3)\left[\begin{array}{l}
b_{1} \\
b_{2} \\
b_{3}
\end{array}\right]=\left[\begin{array}{c}
\left(a_{11} b_{1}+a_{12} b_{2}+0\right) \\
\left(0+0+a_{23} b_{3}\right) \\
\left(a_{31} b_{1}+0+a_{33} b_{3}\right) \\
\left(0+a_{42} b_{2}+0\right)
\end{array}\right]
\end{gathered}
$$

In this example, the first component of the resulting matrix is given by the value $\left(a_{11} b_{1}+a_{12} b_{2}+\right.$ 0 ). Since we already know that the $\mathrm{a}_{13}$ term of matrix A has a value of zero, we really don't need to calculate $\mathrm{a}_{13} \mathrm{~b}_{3}$ term since it will also be 0 . And subsequently, the extra addition calculation of the $a_{13} b_{3}$ term is no longer needed. Two fewer calculations are needed to find the resulting component value. The same will be true for any component of the matrix A that we know ahead 
of time has a value of zero. Each zero component will decrease the number calculations needed by 2: eliminating the need for one multiplication and one addition.

So in the case of multiplying a rectangular $\mathrm{m} \times \mathrm{n}$ matrix, $\mathrm{A}$, that contains $\mathrm{z}$ components with a value of zero, by an $\mathrm{n} x 1$ vector, the total number of calculations needed to perform the operation is $2 \mathrm{~nm}-\mathrm{m}-2 \mathrm{z}$, or equivalently $2(\mathrm{~nm}-\mathrm{z})-\mathrm{m}$.

Since $\mathrm{A}$ is an $\mathrm{n} \mathrm{x}$ m matrix, the total number of matrix components is given by $\mathrm{nm}$. If there are $\mathrm{z}$ components that have a value of zero, that means that the number of components that have a non zero value equals $\mathrm{nm}-\mathrm{z}$. If we let $\mathrm{k}$ equal the number of non zero components of the matrix $\mathrm{A}$, then $\mathrm{k}=\mathrm{nm}-\mathrm{z}$. So the total number of calculations needed to multiply a rectangular $\mathrm{m} \times \mathrm{n}$ matrix, A, which contains k nonzero components, by an $\mathrm{n} x 1$ vector can be re-expressed as $2 \mathrm{k}-\mathrm{m}$. This will be a useful mathematical tool we can use to evaluate some of the reconstruction methods of the CTIS system.

In the following pages we will use this mathematical tool to analyze the number of calculations needed to perform some of the various reconstruction techniques, such as EM and MART. This will be an important analysis because, historically, reconstruction times for CTIS systems have been extremely long, and have limited many of the applications of CTIS. One of the aims of this dissertation work is to try and overcome this reconstruction speed problem. Once we know the total number of calculations needed for each reconstruction method, we can then determine which methods of reconstruction will be faster than others for various conditions.

Ultimately, future CTIS systems may be able to be designed with certain inherent properties that will allow for faster reconstructions. Additionally our methods of system calibration, selected reconstruction methods, specified performance requirements, interpretation of reconstructed data, and post processing of data can be changed in order to allow both future CTIS designs and existing CTIS systems to achieve faster datacube reconstructions.

Before describing some of those approaches, the following pages will examine the number of calculations needed in each step of the EM, MART, and direct inverse reconstruction methods. Each presented reconstruction method is broken into its various steps, with each step consisting of a matrix vector multiplication or an element by element multiplication or division of the components of two vectors. By using the same analysis outlined above, we can determine the number of calculations needed for each step. The sum of the number of required calculations for each step will provide the total number of calculations needed for each reconstruction technique or the total number of calculations needed for each iteration, for the iterative reconstruction techniques. Since some methods will require fewer calculations than others, they may be faster to compute. On each page, a table is provided for each implementation of a reconstruction method outlining the various steps and the total number of required calculations. 


\subsection{Expectation Maximization (EM) Analysis}

EM: $\quad \hat{f}^{(k+1)}=\frac{\hat{f}^{(k)}}{\sum_{m=1}^{M} H_{m n}} H^{T}\left(\frac{g}{H \hat{f}^{(k)}}\right)$

$H \hat{f}^{(k)} \Rightarrow \operatorname{pixels}\left[\begin{array}{c}\text { voxels } \\ \end{array}\left[^{1}\right]\right.$ voxels

Result dimensions: (pixels x 1)

$\frac{g}{\left[\hat{f}^{(k)}\right]} \Rightarrow \frac{\left[{ }^{1}\right] \text { pixels }}{\left[{ }^{1}\right] \text { pixels }} \quad$ element by element divisions

Result dimensions: (pixels x 1)

Step 3)

$\left\{H^{T}\left(\frac{g}{\left[H \hat{f}^{(k)}\right]}\right)\right\} \Rightarrow$ voxels

Result dimensions: (voxels x 1)

Step 4)

$\left(\frac{\left\{H^{T}\left(\frac{g}{\left[H \hat{f}^{(k)}\right]}\right)\right\}}{\sum_{m=1}^{M} H_{m n}} \Rightarrow \frac{\left.{ }^{1}\right]}{{ }_{\text {voxels }}^{1}}\right.$

Result dimensions: (voxels x 1)

Step 5)

$\hat{f}(k) \frac{\left\{H^{T}\left(\frac{g}{\left[H \hat{f}^{(k)}\right]}\right)\right\}}{\sum_{m=1}^{M} H_{m n}} \Rightarrow\left[\begin{array}{l}1 \\ \text { voxels } \times\left[^{1}\right] \text { voxels element by element multiplications }\end{array}\right.$

Result dimensions: (voxels x 1)

Number of calculations needed for Step $5=\mathrm{v}$

Where $\mathrm{p}=\#$ of focal plane pixels, $\mathrm{v}=\#$ of datacube voxels, and $\mathrm{k}=\#$ of nonzero elements in the system $\mathrm{H}$ matrix

Total Number of Calculations Needed

Per Iteration of EM $=\mathbf{4 k}+\mathbf{v}$ 
7.8 Multiplicative Algebraic Reconstruction Technique (MART) Analysis

Implementation 1: Two Matrix vector multiplications for $\mathrm{H}^{\mathrm{T}} \mathbf{H f}$

$$
\text { MART: } \quad \hat{f}^{(k+1)}=\hat{f}^{(k)}\left(\frac{H^{T} g}{H^{T} H \hat{f}^{(k)}}\right)
$$

$$
\text { Step 1) } \quad H^{T} g \Rightarrow \text { voxels }\left[\begin{array}{c}
\text { pixels } \\
{[}
\end{array}\right] \text { pixels }
$$

NOTE: This initial calculation only needs to be done ONCE at the beginning of the reconstruction Result dimensions: (voxels x 1)

Number of calculations needed for Step $1=2 \mathrm{k}-\mathrm{v}$

Step 2)

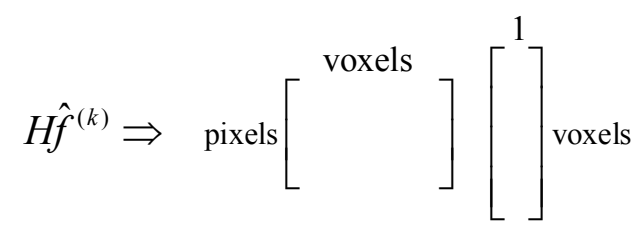

Result dimensions: (pixels x 1)

Number of calculations needed for Step $2=2 \mathrm{k}-\mathrm{p}$

$H^{T}\left(H \hat{f}^{(k)}\right) \Rightarrow$ voxels $\left[\begin{array}{c}\text { pixels } \\ \end{array}\right]\left[\begin{array}{l}1 \\ \end{array}\right]$ pixels

Result dimensions: (voxels x 1)

Number of calculations needed for Step $3=2 \mathrm{k}-\mathrm{v}$

$\left(\frac{H^{T} g}{H^{T} \hat{f}^{(k)}}\right) \Rightarrow \frac{\left[\begin{array}{l}1 \\ {[}\end{array}\right] \text { voxels }}{\left[\begin{array}{l}1 \\ \text { voxels }\end{array}\right.}$

Result dimensions: (voxels x 1)

$\hat{f}^{(k)}\left(\frac{H^{T} g}{H^{T} H \hat{f}^{(k)}}\right) \Rightarrow\left[{ }^{1}\right]$ voxels $\times\left[^{1}\right]_{\text {voxels }} \quad$ element by element multiplications

Result dimensions: (voxels x 1)

Number of calculations needed for Step $5=\mathrm{v}$

$$
\begin{gathered}
\text { Total Number of Calculations Needed } \\
\text { Per Iteration of MART }=\mathbf{4 k}+\mathbf{v}-\mathbf{p} \\
\text { Initial Start of MART }=\mathbf{2} \mathbf{k}-\mathbf{v}
\end{gathered}
$$




\subsection{Multiplicative Algebraic Reconstruction Technique (MART) Analysis} Implementation 2: A single Matrix vector multiplication for $\mathrm{H}^{\mathrm{T}} \mathbf{H f}$

$$
\text { MART: } \quad \hat{f}^{(k+1)}=\hat{f}^{(k)}\left(\frac{H^{T} g}{H^{T} H \hat{f}^{(k)}}\right)
$$

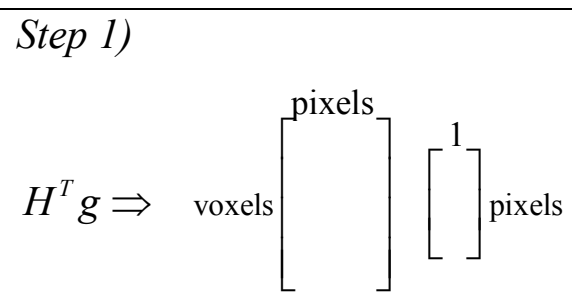

NOTE: This initial calculation only needs to be done ONCE at the beginning of the reconstruction

Result dimensions: (voxels x 1)

$\left(H^{T} H\right) \hat{f}^{(k)} \Rightarrow$ voxels $\left[\begin{array}{c}\text { voxels } \\ \end{array}\right]\left[\begin{array}{c}1 \\ \end{array}\right]$ voxels

NOTE: The term $\mathrm{H}^{\mathrm{T}} \mathrm{H}$ is determined before hand.

Knowing just the number of zero elements in $\mathrm{H}$, does not tell us the number of zero elements in $\mathrm{H}^{\mathrm{T}} \mathrm{H}$.

Result dimensions: (voxels x 1)

$\left(\frac{H^{T} g}{H^{T} H \hat{f}^{(k)}}\right) \Rightarrow \frac{\left[^{1}\right]_{\text {voxels }}}{\left[\begin{array}{l}1 \\ \text { voxels }\end{array}\right.}$ element by element divisions

Result dimensions: (voxels x 1)

$\hat{f}^{(k)}\left(\frac{H^{T} g}{H^{T} H \hat{f}^{(k)}}\right) \Rightarrow\left[\left[^{1}\right]\right.$ voxels $\times\left[{ }^{1}\right]$ voxels element by element multiplications

Result dimensions: (voxels x 1)

Number of calculations needed for Step $5=\mathrm{V}$

Where $\mathrm{p}=\#$ of focal plane pixels, $\mathrm{v}=\#$ of datacube voxels, and $\mathrm{k}=\#$ of nonzero elements in the system $\mathrm{H}$ matrix

Total Number of Calculations Needed

Per Iteration of MART $=\mathbf{2} \mathbf{v}^{\mathbf{2}}+\mathbf{v}$

Initial Start of MART $=\mathbf{2} \mathbf{k}-\mathbf{v}$ 


\subsection{Newly Proposed Direct Inverse Reconstruction Technique: Analysis}

$$
\text { Direct Left Inverse: } f=H_{l e f t}^{-1} g
$$

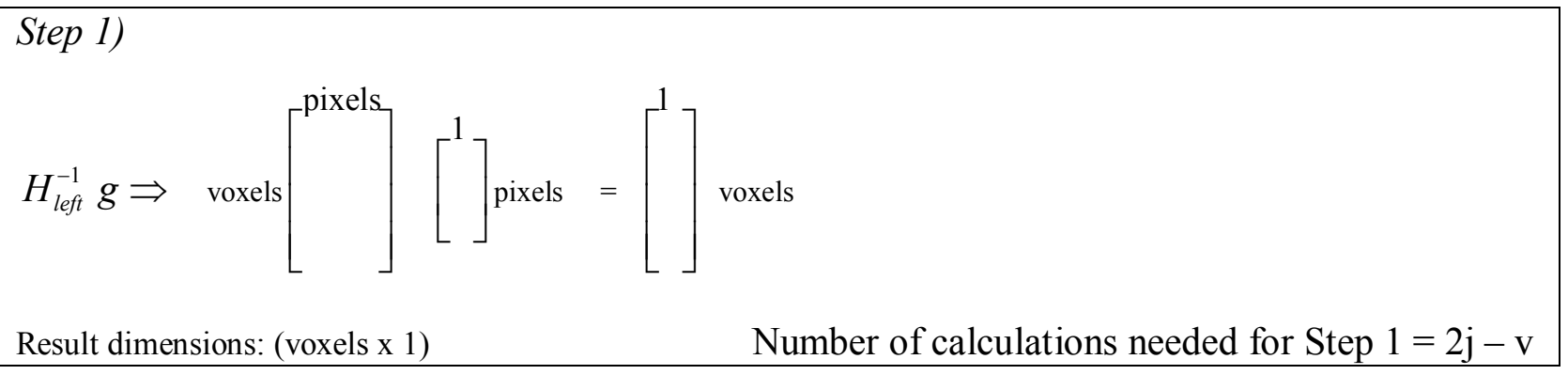

Where $\mathrm{p}=\#$ of focal plane pixels, $\mathrm{v}=\#$ of datacube voxels, $\mathrm{k}=\#$ of nonzero elements in the system $\mathrm{H}$ matrix, and $\mathrm{j}=\#$ of nonzero elements in the matrix $\mathrm{H}^{-1}$ left

\section{Total Number of Calculations Needed Direct Left Inverse Reconstruction Technique $=\mathbf{2} \mathbf{j}-\mathbf{V}$}

\subsection{Evaluating Which Reconstruction Methods are Faster}

Now that we have a mathematical description of the total number of calculations needed for each reconstruction method, we can determine which methods will be faster than others for various conditions. In terms of comparing the speed of these operations, lets first compare the iterative methods starting with MART. There are two implementations of MART, one where the $\mathrm{H}^{\mathrm{T}} \mathrm{Hf}$ term is calculated through two separate matrix vector multiplications and the other where the term is determined from a single multiplication of the square matrix $\left(\mathrm{H}^{\mathrm{T}} \mathrm{H}\right)$ and the datacube vector, $f$. The analysis of the reconstruction techniques in the following pages will answer questions such as: Which one of the two implementations of MART is faster/requires the fewer number of calculations? Under what conditions will EM be faster than MART? Are there any cases where the direct left inverse technique will be faster than the iterative approaches?

\subsection{Comparing Speed of Different Implementations of MART}

There are two possible implementations of MART:
1) $\mathrm{H}^{\mathrm{T}}\left[\mathrm{Hf}^{(\mathrm{k})}\right]$
2) $\left[\mathrm{H}^{\mathrm{T}} \mathrm{H}\right] \mathrm{f}^{(\mathrm{k})}$
two separate matrix multiplications one matrix multiplication

Even though we know the number of zero elements in $\mathrm{H}$, based on just this information we can not always state the number of zeros in $\mathrm{H}^{\mathrm{T}} \mathrm{H}$. $\mathrm{H}^{\mathrm{T}} \mathrm{H}$ is by no means guaranteed to have a number of nonzero elements related to the number of nonzero elements of $\mathrm{H}$. We will use the general approach to determine the number of calculations needed to multiply this square matrix times the vector $\mathrm{f}^{(\mathrm{k})}$. 
Total Number of Calculations needed for Implementation 1, with two separate matrix multiplications:

- $4 \mathrm{k}+\mathrm{v}-\mathrm{p} \quad$ per iteration

- $2 \mathrm{k}-\mathrm{v} \quad$ initial

Total Number of Calculations needed for Implementation 2, with one matrix multiplications:

- $2 \mathrm{v}^{2}+\mathrm{v} \quad$ per iteration

- $2 \mathrm{k}-\mathrm{v} \quad$ initial

Since the number of initial calculations are the same for both implementations, we only need to consider the number of calculations per iteration: $\left(2 v^{2}+v\right)$ vs. $(4 k+v-p)$.

The number of nonzero values, $\mathrm{k}$, in the matrix $\mathrm{H}$ is effectively given by the number of lit pixels in a given calibration image times the number of calibration images, $v$, assuming that the number of lit pixels is the same in all of the calibration images.

Assume that $\mathrm{k}=\mathrm{Lv}$, where $\mathrm{L}$ is the number of lit pixels in a calibration image.

$2 \mathrm{v}^{2}+\mathrm{v}$ vs. $4 \mathrm{k}+\mathrm{v}-\mathrm{p} \quad$ (subtract $\mathrm{v}$ from both sides)

$2 \mathrm{v}^{2}$ vs. $4 \mathrm{k}-\mathrm{p}$

$2 v^{2}$ vs. $4 \mathrm{Lv}-\mathrm{p} \quad$ (substitute in $\mathrm{k}=\mathrm{Lv}$ )

Consider the case where $\mathrm{L}=(\mathrm{v} / 2)$. In this case the number of lit pixels in each calibration image is equal to half the number of voxels in the system.

$2 \mathrm{v}^{2}$ vs. $4(\mathrm{v} / 2) \mathrm{v}-\mathrm{p}$

$2 \mathrm{v}^{2}$ vs. $2 \mathrm{v}^{2}-\mathrm{p}$

$2 v^{2}>2 v^{2}-p$

Even in the case where $\mathrm{L}=(\mathrm{v} / 2)$, implementation 1 of MART, using two separate matrix multiplications will require fewer calculations to complete than using one matrix multiplication. In general L will be much smaller than (v/2) because we are trying to make the $\mathrm{H}$ matrix as sparse as possible to increase the speed of reconstructions. So using two separate matrix multiplications, which take advantage of the zero values of the sparse $\mathrm{H}$ matrix, is the faster way to implement the Multiplicative Algebraic Reconstruction Technique (MART). 


\subsection{Comparing Reconstruction Speed for EM and MART}

Note that this analysis is not taking into account the quality of reconstructions, it is merely aimed at determining for a given number of iterations, which technique would be completed first. The number of calculations needed for each technique are as follows:

EM requires: $\quad 4 \mathrm{k}+\mathrm{v} \quad$ calculations per iteration

MART requires: $\quad 4 \mathrm{k}+\mathrm{v}-\mathrm{p} \quad$ calculations per iteration and $2 \mathrm{k}-\mathrm{v} \quad$ initial calculations

where $\mathrm{v}=\#$ of voxels in the system, $\mathrm{p}=\#$ of pixels, and $\mathrm{k}=\#$ of nonzero values in the system matrix, $\mathrm{H}$

The total number of calculations needed to complete I iterations:

EM: $\quad(4 \mathrm{k}+\mathrm{v}) \mathrm{I} \quad=(4 \mathrm{kI}+\mathrm{vI})$

MART: $\quad(4 \mathrm{k}+\mathrm{v}-\mathrm{p}) \mathrm{I}+2 \mathrm{k}-\mathrm{v}=(4 \mathrm{kI}+\mathrm{vI})-\mathrm{pI}+2 \mathrm{k}-\mathrm{v}$

The term, $-\mathrm{pI}+2 \mathrm{k}-\mathrm{v}$, will determine which method will be faster for a fixed number of iterations, I. If $\mathrm{pI}$ is greater $2 \mathrm{k}-\mathrm{v}$, MART will be faster. If $\mathrm{pI}$ is less than $2 \mathrm{k}-\mathrm{v}$, EM will be faster.

EM faster if: $\quad I<\frac{2 k-v}{p}, \quad$ MART faster if: $\quad I>\frac{2 k-v}{p}$

Assuming that the number of nonzero values, $\mathrm{k}$, in the matrix $\mathrm{H}$ is effectively given by the number of lit pixels in a given calibration image, $\mathrm{L}$, times the number of calibration images, $\mathrm{v}$, we have $\mathrm{k}=\mathrm{Lv}$.

EM faster if: $\quad I<\frac{v}{p}(2 L-1)$

MART faster if: $\quad I>\frac{v}{p}(2 L-1)$

In general for higher resolution constructed systems, $v>p$, implying that the system will have more unknowns (voxels) than measurements (recorded pixel values). It may be reasonable to say that the smallest value of $\mathrm{v} / \mathrm{p}$ would be 1 . Also the smallest $\mathrm{L}$ value would correspond to only a single pixel being lit in each of the diffraction orders in each of the calibration images, resulting in $\mathrm{L}=$ the number of diffraction orders in the system. If we consider just a $3 \times 3$ diffraction pattern for this case, $\mathrm{L}$ would equal 9 . In this case, trying to make the right hand side of the inequality as small as possible, we see that EM is faster if, $I<17$.

As a somewhat general rule for higher resolution systems, if you are completing 15 iterations or less, EM will be the faster reconstruction method. If you are completing more than 15 iterations, you can use the equations above to determine whether EM or MART will be faster. For a lower resolution CTIS, use the equations above to determine which is faster. 


\subsection{Comparing Reconstruction Speed for EM and Direct Left Inverse}

The total number of calculations needed to complete reconstructions for I iterations of EM and the direct left inverse technique are:

$$
\text { EM: } \quad(4 \mathrm{k}+\mathrm{v}) \mathrm{I}
$$

DIRECT INVERSE: $(2 \mathrm{j}-\mathrm{v})$

Where $\mathrm{v}=\#$ of datacube voxels, $\mathrm{k}=\#$ of nonzero elements in the system $\mathrm{H}$ matrix, and $\mathrm{j}=\#$ of nonzero elements in the matrix $\mathrm{H}^{-1}$ left.

Reconstructions using EM will be faster when $(4 k+v) I<(2 j-v)$.

However, there is a limit to the number of iterations of EM that can be calculated before the direct left inverse technique would finish first.

EM will be faster if: $\quad I<\frac{(2 j-v)}{(4 k+v)}$

If a given matrix, $\mathrm{H}$, is very sparse, this by no means implies that the left inverse of $\mathrm{H}$ will also be sparse. So the number of nonzero elements, $\mathrm{j}$, in the left inverse of the $\mathrm{H}$ matrix will be significantly greater than the number of nonzero elements, $\mathrm{k}$, in the $\mathrm{H}$ matrix itself. Because of this EM should be faster if only a small number of iterations are required. This is illustrated in the example table below for various simple CTIS systems that have a spatial resolution of $6 \times 6$ pixels and various size wavelength bands.

\begin{tabular}{|l|r|r|c|c|}
\hline \multicolumn{5}{|c|}{$\begin{array}{c}\text { Comparison of Direct Left Inverse to EM for } \\
\text { Simple CTIS systems with 3x3 diffraction pattern }\end{array}$} \\
\hline datacube & Voxels & \multicolumn{1}{c|}{$\mathrm{J}$} & \multicolumn{1}{c|}{ K } & $(2 \mathrm{j}-\mathrm{v}) /(4 \mathrm{k}+\mathrm{v})$ \\
\hline $6 \times 6 \times 5$ & 180 & 111060 & 1620 & $\mathbf{3 3 . 3 2}$ \\
\hline $6 \times 6 \times 10$ & 360 & 376200 & 3240 & $\mathbf{5 6 . 4 6}$ \\
\hline $6 \times 6 \times 20$ & 720 & 1442160 & 6480 & $\mathbf{1 0 8 . 2 4}$ \\
\hline $6 \times 6 \times 30$ & 1080 & 3247560 & 9720 & $\mathbf{1 6 2 . 5 1}$ \\
\hline
\end{tabular}

Table 4: $\mathrm{H}$ and $\mathrm{H}$ inverse properties of sample CTIS systems

In the first example, reconstructions of the datacube using EM will be faster than the direct left inverse technique, as long as the number of iterations of EM is less than or equal to 33. In the remaining examples, systems that have an increased number of wavelength bands will be able to compute more iterations of EM faster than the direct left inverse technique because the number of nonzero values in the $\mathrm{H}$ matrix is not increasingly nearly as fast as the number of nonzero values in $\mathrm{H}^{-1}$ left as more bands are added.

Note that the values listed in the table are using a "simple" representation of the CTIS system. In actual systems, things can become a bit more complicated. Certain parameters, like the value of 
$\mathrm{k}$, can be quite different for actual systems compared to the simple representation. There may be cases where the direct inverse will be faster, but if certain changes are made to the calibration, reconstruction, and possibly post processing of the data, the value of $\mathrm{k}$ for actual systems can be made much smaller, closer resembling the simple representation. These changes will allow reconstructions using EM to be faster than the direct left inverse in nearly all cases. Details on how this would be implemented will be presented later in the chapter.

So as a general rule, multiple iterations of EM can be completed in the time it takes to finish a single direct left inverse reconstruction for a CTIS system.

Before moving on to discuss the effect of parallel processing methods for reconstructions it is worthwhile to briefly note a few properties of the reconstruction results from both the EM and direct left inverse techniques. Recall that this analysis of the number of computations needed for each reconstruction technique has not taken into account the quality of reconstructions. When considering the quality of the results, the direct left inverse is capable of producing a "perfect" error free reconstruction in simulated simple CTIS systems, where the direct left inverse exists. In those same simulated CTIS systems, we see that the results from EM will asymptotically approach the direct left inverse results as more iterations of EM are calculated. The largest changes, or decreases in errors of the reconstruction, occur in the first few iterations of EM, but even as the number of iterations is dramatically increased it will still have a reconstruction error that is greater than the direct left inverse result. Note that these effects can change in physical implemented CTIS systems as described below.

While considering the speed of CTIS reconstructions using the various reconstruction techniques it is useful to keep in mind a sense of when the results are "good enough." And while the asymptotic approach of EM to the direct left inverse results is true for simulated systems, noise and other factors can change these effects in implemented systems. In the direct left inverse case the conditioning of the left inverse matrix will relate how noise in the original image is amplified in the reconstruction data, and will vary depending upon the system and calibration/H matrix. Nathan Hagen has also shown that when using EM with noisy input images there is a limit to the number of iterations of EM that provide improvements of your datacube estimate. After a given point, more iterations of EM will only amplify the effect of the noise and increase the error in the reconstruction results. ${ }^{22}$ These effects should be evaluated for each CTIS system and its particular application. When determining the optimal method of selecting reconstruction techniques and corresponding reconstruction speeds it is useful to have an idea of when the reconstruction results are good enough.

\subsection{How parallel processing of the CTIS H matrix multiplications could increase speeds}

The previous comparisons illustrate that in all of the examined reconstruction techniques the most computationally intensive steps always involve performing matrix vector multiplications with a very large matrix. Also note that this comparison of reconstructions does NOT take into account any possible parallel processing of the calculations. The results merely describe the total number of calculations needed to complete each reconstruction method, which is directly related to the speed of reconstruction when each mathematical operation is done sequentially. By 
performing these calculations simultaneously, spread out over multiple processors, the speed of reconstructions can be dramatically increased. With the goal of increasing the speed of reconstructions, the following methods of performing parallel computations will be investigated as possible ways to quickly finish those computationally intensive tasks:

1) Parallel processing of matrix vector multiplication that separate multiplications and additions into parallel operations

2) Parallel processing of matrix vector multiplication that separate multiplications and additions into parallel operations taking into account sparse nature of the matrix

3) Parallel processing of matrix vector multiplication using a fused multiply and add operation

4) Parallel processing of matrix vector multiplication using a fused multiply and add operation taking into account sparse nature of the matrix

Each of these methods will be applied to both the iterative EM reconstruction technique and the direct left inverse technique to see how each method could be implemented, how quickly the reconstruction could be completed, and how many parallel processors would be needed.

In order to determine how these methods could be implemented, let's begin by looking at method 1 and how the first step of the EM calculation could be run in a parallel fashion to increase the speed of reconstructions.

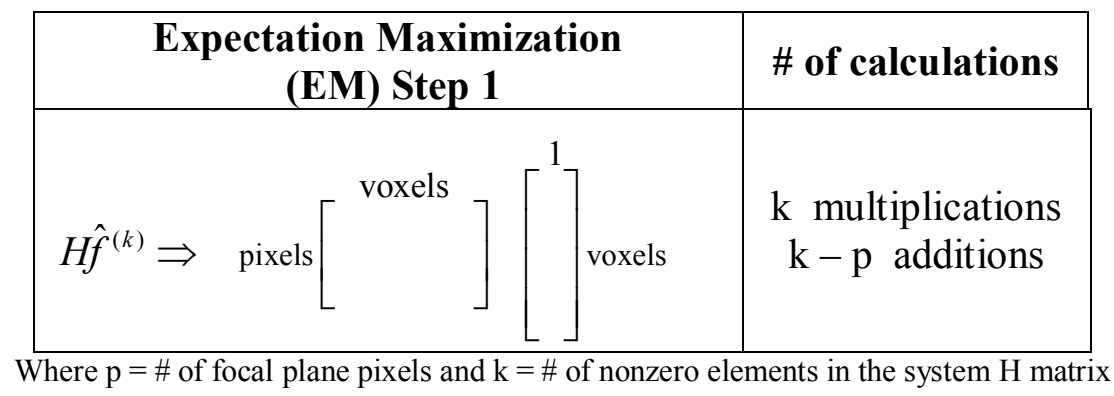

If you had k separate parallel processors working together to perform the calculation, all of the necessary multiplications could be done simultaneously in a single calculation cycle/processor clock. So in the example matrix vector multiplication below, one processor would calculate the product of $a_{11} b_{1}$. A separate would calculate $a_{12} b_{2}$, another $a_{13} b_{3}$, another $a_{32} b_{2}$, etc. By using k $=12$ processors, all of the required multiplications could be computed simultaneously in a single calculation cycle/processor clock.

$$
\begin{gathered}
(\mathrm{n} \text { columns, } \mathrm{n}=3) \\
\left(\begin{array}{lll}
a_{11} & a_{12} & a_{13} \\
a_{21} & a_{22} & a_{23} \\
a_{31} & a_{32} & a_{33} \\
a_{41} & a_{42} & a_{43}
\end{array}\right] \quad(\mathrm{n}=3)\left[\begin{array}{l}
b_{1} \\
b_{2} \\
b_{3}
\end{array}\right]=\left[\begin{array}{c}
\left(a_{11} b_{1}+a_{12} b_{2}+a_{13} b_{3}\right) \\
\left(a_{21} b_{1}+a_{22} b_{2}+a_{23} b_{3}\right) \\
\left(a_{31} b_{1}+a_{32} b_{2}+a_{33} b_{3}\right) \\
\left(a_{41} b_{1}+a_{42} b_{2}+a_{43} b_{3}\right)
\end{array}\right]
\end{gathered}
$$


However, in order to complete the matrix vector multiplication, all of these products need to be added in a particular manner to produce the components of the resulting vector. In the example, the first component of the vector will require two addition operations: the first operation being $\left(a_{11} b_{1}+a_{12} b_{2}\right)$ and the second operation being the sum of that result with $a_{13} b_{3}$. Note that because the second addition operation is dependent upon the results of the first, the required addition operations cannot be made parallel nearly as efficiently as the multiplication operations.

The calculation of all of the additions can be made parallel by separating the products for each component into groups of two and then adding each group simultaneously. This process would be repeated until only a single value remained for each component of the vector. Unlike the operation to determine the products, completing all of the additions will require multiple calculation cycles to complete.

In the example two additions must be performed for each component of the resulting vector. In the general case the maximum number of additions needed for a single component is (n-1), where $\mathrm{n}$ is the number of columns of the matrix. When using the above method to make this process parallel, the maximum number of calculations cycles/processor clocks needed to complete the calculations would equal the exponent value of the closest integer power of 2 that is greater than (n-1). For example, if there are 10 separate numbers that need to be added to determine a component, the number of cycles of parallel additions needed to complete the calculation is 4 . (The closest power of 2 that is greater than 9 is $2^{4}=16$.) Starting with 10 values, 5 additions will be made in the first step, leaving 5 remaining values. In the second step, 2 additions will be made, leaving 3 remaining values. In the third step, 1 addition will be made, leaving 2 values. In the final fourth step, a single addition would be made for those final 2 values, with the result equaling the component value. Using $\mathrm{k}$ separate processors, this same parallel process could be carried out for all $\mathrm{m}$ components of the final vector simultaneously.

Method 2 is extremely similar, but will take advantage of the sparse nature of the matrix. Consider the following sparse example that has $\mathrm{k}=6$ nonzero elements in the matrix:

$$
\begin{gathered}
\text { (n columns, } \mathrm{n}=3) \quad(\mathrm{c}=1) \\
(\mathrm{m} \text { rows, } \mathrm{m}=4)\left[\begin{array}{ccc}
a_{11} & a_{12} & 0 \\
0 & 0 & a_{23} \\
a_{31} & 0 & a_{33} \\
0 & a_{42} & 0
\end{array}\right] \quad(\mathrm{n}=3)\left[\begin{array}{c}
b_{1} \\
b_{2} \\
b_{3}
\end{array}\right]=\left[\begin{array}{c}
\left(a_{11} b_{1}+a_{12} b_{2}+0\right) \\
\left(0+0+a_{23} b_{3}\right) \\
\left(a_{31} b_{1}+0+a_{33} b_{3}\right) \\
\left(0+a_{42} b_{2}+0\right)
\end{array}\right]
\end{gathered}
$$

Again, if you had k separate parallel processors working together to perform the calculation, all of the necessary multiplications could be done simultaneously in a single cycle. However in this case the number of necessary additions is reduced. Instead of being related to the number of columns in the matrix, the number of needed additions will be related to the maximum number of nonzero elements in a single row of the matrix. For a CTIS system, this is the same as the maximum \# of voxels that map to a single pixel when performing an $\mathrm{H}$ times $\mathrm{f}$ calculation, and the maximum \# of lit pixels in a voxel's calibration image when performing an $H$ transpose times g operation. Using the same parallel addition steps, the number of cycles needed to complete the 
calculations now equals the exponent value of the closest power of 2 that is greater than the maximum number of nonzero elements in a single row of the matrix minus 1 .

Method 3 uses a processing technique called Fused Multiply and Add (FMA), which calculates the product of two numbers and adds that product to an accumulator value. There is a speed benefit to the FMA implementation in modern graphics processing units (GPU's) because it allows both the multiplication and addition operation of single precision floating point values to be performed in a single clock while still retaining full precision in the intermediate stage. Upcoming graphics processors, such as the Fermi GPU from NVIDIA, will contain 512 processing cores allowing 512 FMA operations to be performed per clock.

$$
\begin{gathered}
(\mathrm{n} \text { columns, } \mathrm{n}=3) \quad(\mathrm{c}=1) \\
\left(\begin{array}{lll}
a_{11} & a_{12} & a_{13} \\
a_{21} & a_{22} & a_{23} \\
a_{31} & a_{32} & a_{33} \\
a_{41} & a_{42} & a_{43}
\end{array}\right] \quad(\mathrm{n}=3)\left[\begin{array}{l}
b_{1} \\
b_{2} \\
b_{3}
\end{array}\right]=\left[\begin{array}{c}
\left(a_{11} b_{1}+a_{12} b_{2}+a_{13} b_{3}\right) \\
\left(a_{21} b_{1}+a_{22} b_{2}+a_{23} b_{3}\right) \\
\left(a_{31} b_{1}+a_{32} b_{2}+a_{33} b_{3}\right) \\
\left(a_{41} b_{1}+a_{42} b_{2}+a_{43} b_{3}\right)
\end{array}\right]
\end{gathered}
$$

When applying Fused Multiply and Add to the example matrix vector multiplication above, the calculation can be completed in just 3 cycles using only 4 four processors. Each processor would perform the calculations for a specific component of the resulting vector. The processor assigned to the first component of the vector would perform the following operations during each cycle:

Fused Multiply and Add (FMA): $\quad a \leftarrow\{a\}+(b \times c)$

Cycle 1: $\quad a \leftarrow\left(a_{11} \times b_{1}\right)$

Cycle 2: $\quad a \leftarrow\left\{a_{11} b_{1}\right\}+\left(a_{12} \times b_{2}\right)$

Cycle 3: $\quad a \leftarrow\left\{a_{11} b_{1}+a_{12} b_{2}\right\}+\left(a_{13} \times b_{3}\right)$ multiplication operation

FMA operation

FMA operation

In the general case when using FMA to calculate the resulting vector when multiplying an $\mathrm{m} \times \mathrm{n}$ matrix with an $\mathrm{n} \times 1$ column vector, the number of processors needed will equal the \# of rows of the matrix, and the number of processor cycles need will equal the \# of columns of the matrix.

Method 4 will also use Fused Multiply and Add, but will take advantage of the sparse nature of the matrix. Consider the example below:

$$
\begin{gathered}
(\mathrm{n} \text { columns, } \mathrm{n}=3) \quad(\mathrm{c}=1) \\
(\mathrm{m} \text { rows, } \mathrm{m}=4)\left[\begin{array}{ccc}
a_{11} & a_{12} & 0 \\
0 & 0 & a_{23} \\
a_{31} & 0 & a_{33} \\
0 & a_{42} & 0
\end{array}\right] \quad(\mathrm{n}=3)\left[\begin{array}{c}
b_{1} \\
b_{2} \\
b_{3}
\end{array}\right]=\left[\begin{array}{c}
\left(a_{11} b_{1}+a_{12} b_{2}+0\right) \\
\left(0+0+a_{23} b_{3}\right) \\
\left(a_{31} b_{1}+0+a_{33} b_{3}\right) \\
\left(0+a_{42} b_{2}+0\right)
\end{array}\right]
\end{gathered}
$$


This calculation can be completed in just 2 cycles using 4 processors by assigning each processor to a specific component of the resulting vector. In general the number of cycles needed will be equal to the maximum \# of nonzero elements in a row of the matrix, and number of processors needed will equal the $\#$ of rows of the matrix.

The following pages will provide details on how each of these methods can be applied to the EM reconstruction technique and the direct left inverse reconstruction technique. The analysis will compare the speed/total number of cycles needed to complete the reconstruction as well as the number of processors needed to perform the calculations for each method. Specific numbers will be presented for an example CTIS system to illustrate the relative speed and computing hardware needed, as well as details for a generalized CTIS system.

Again, each implementation of the reconstruction method will be broken into steps. For the given parallel processing method, the number of processors and calculation cycles needed to complete each step will be listed. By summing the calculation cycles for all of the steps we can compare the total number of calculation cycles needed for each parallel processing method and reconstruction technique. We can also compare the number of processors needed for each implementation. The following pages provide tables describing each method of parallel processing for each reconstruction technique indicating the total number of processors and calculations cycles needed for each reconstruction iteration. For each reconstruction method, a separate page will compare the four parallel processing methods providing the details for a general CTIS system and a specific example CTIS system with a $3 \times 3$ diffraction order pattern and a $6 \times 6 \times 30$ datacube. 


\subsection{EM using Parallel Method 1}

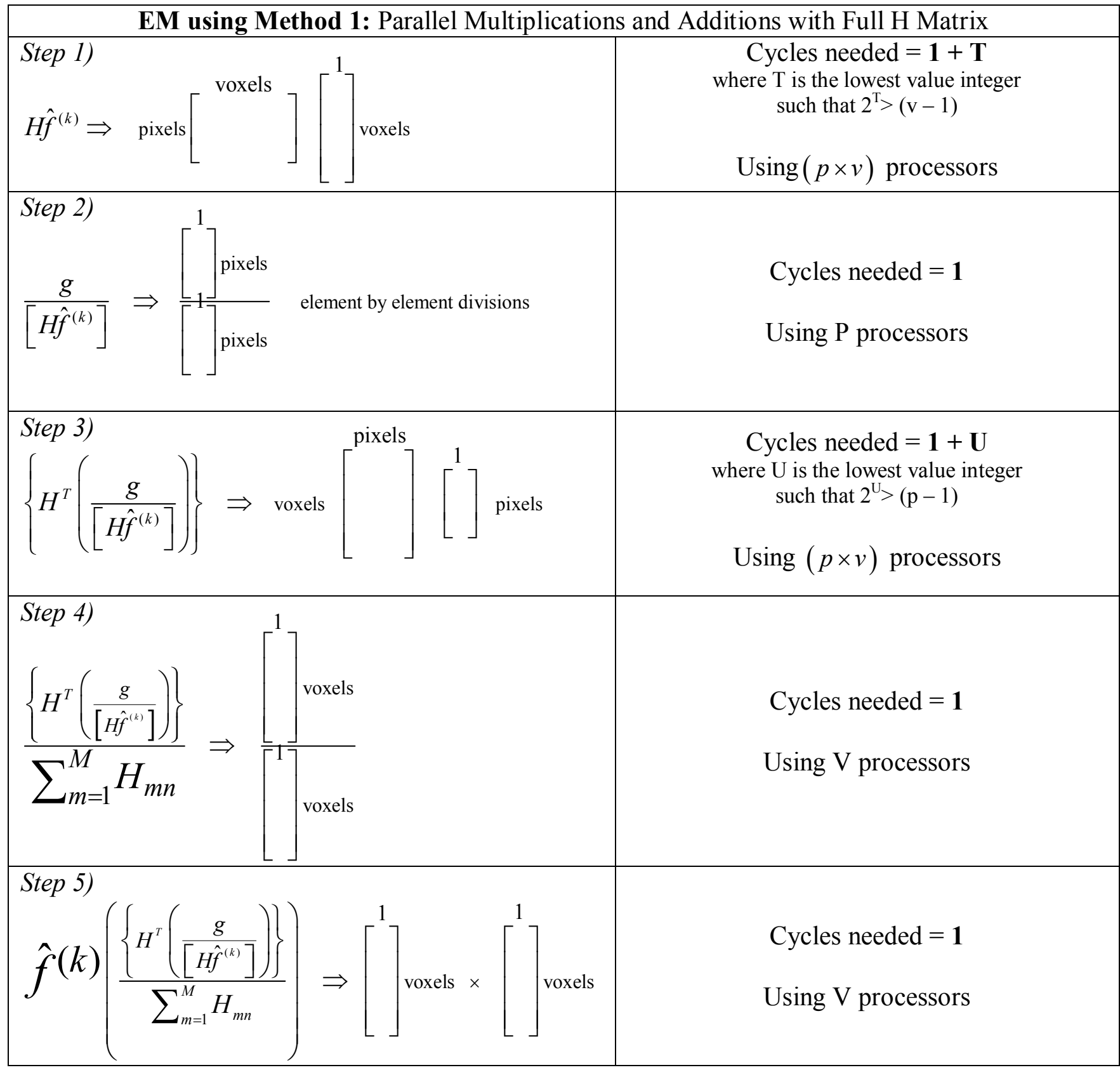

$\mathrm{p}=\#$ of focal plane pixels and $\mathrm{v}=\#$ of datacube voxels. Note that pixels that are never lit by any voxel are not counted in the $\mathrm{p}$ value.

Total Number of Cycles Needed Per Iteration of EM $=\mathbf{5}+\mathbf{T}+\mathbf{U}$ Total Processors Needed $=(\mathbf{P} \mathbf{x} \mathbf{V})$

Where $T$ is the lowest value integer such that $2^{\mathrm{T}}>(\mathrm{v}-1)$ and $\mathrm{U}$ is the lowest value integer such that $2^{\mathrm{U}}>(\mathrm{p}-1)$. 


\subsection{EM using Parallel Method 2}

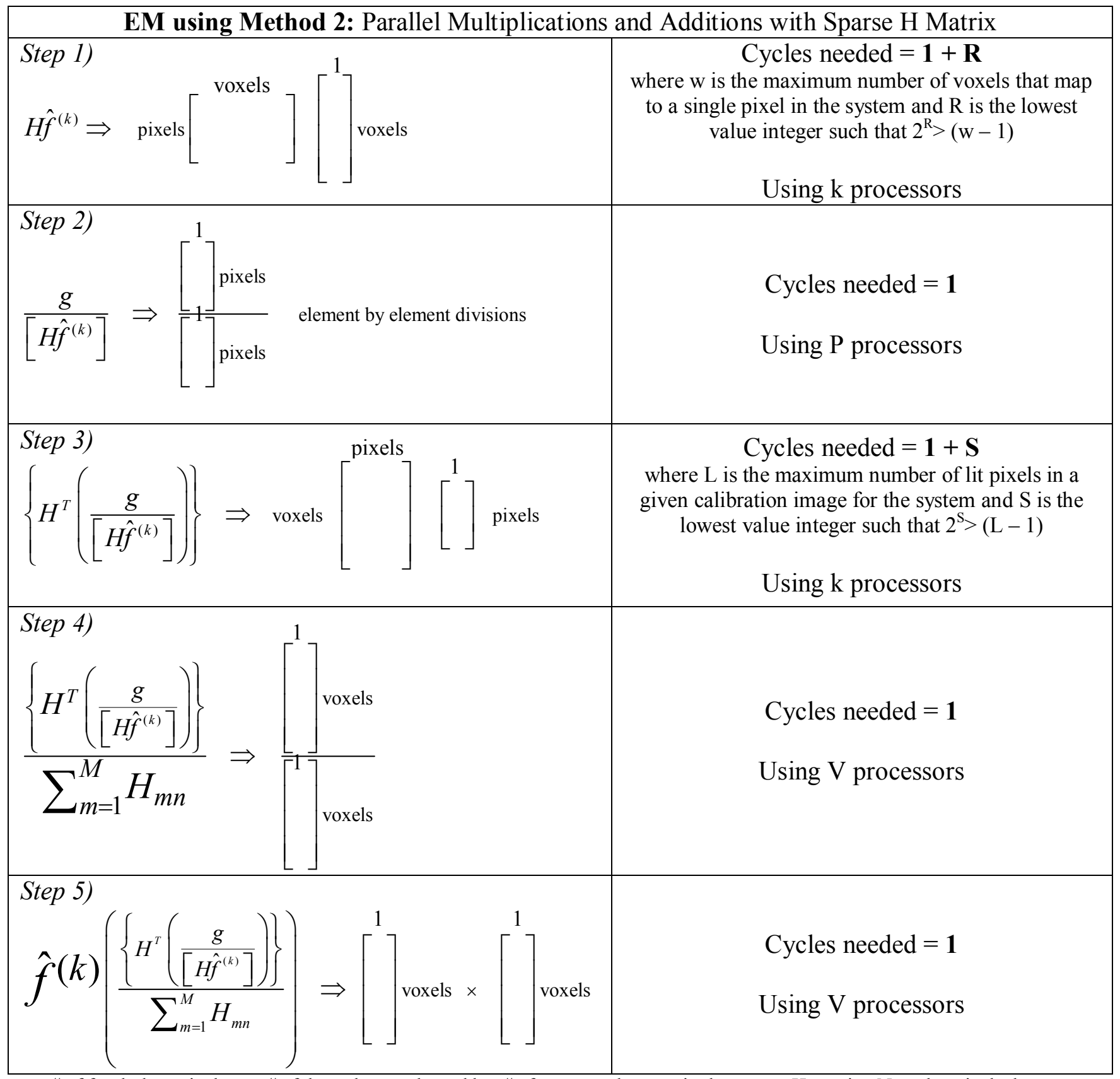

$\mathrm{p}=\#$ of focal plane pixels, $\mathrm{v}=\#$ of datacube voxels, and $\mathrm{k}=\#$ of nonzero elements in the system $\mathrm{H}$ matrix. Note that pixels that are never lit by any voxel are not counted in the $p$ value.

\section{Total Number of Cycles Needed Per Iteration of EM $=\mathbf{5}+\mathbf{R}+\mathbf{S}$ \\ Total Processors Needed $=\mathbf{k}$}

Where $\mathrm{w}$ is the maximum number of voxels that map to a single pixel in the system, $\mathrm{R}$ is the lowest value integer such that $2^{\mathrm{R}}>(\mathrm{w}-1)$, $\mathrm{L}$ is the maximum number of lit pixels in a given calibration image for the system, and $\mathrm{S}$ is the lowest value integer such that $2^{\mathrm{S}}>(\mathrm{L}-1)$. 


\subsection{EM using Parallel Method 3}

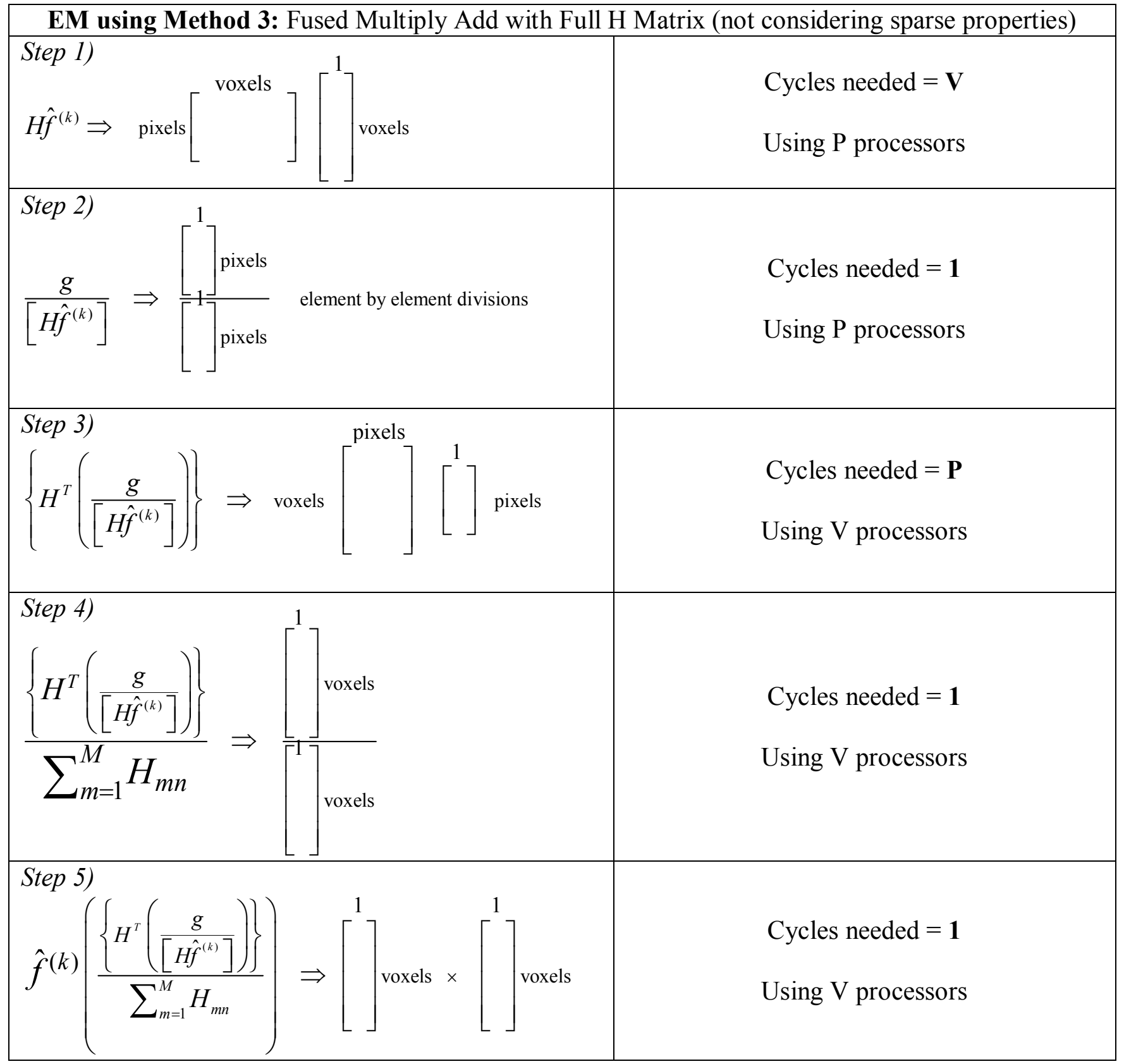

$\mathrm{p}=\#$ of focal plane pixels and $\mathrm{v}=\#$ of datacube voxels. Note that pixels that are never lit by any voxel are not counted in the $\mathrm{p}$ value.

Total Number of Cycles Needed Per Iteration of EM $=\mathbf{3}+\mathbf{V}+\mathbf{P}$

Total Processors Needed $=\mathbf{V}$ or $\mathbf{P}$, whichever is greater 


\subsection{EM using Parallel Method 4}

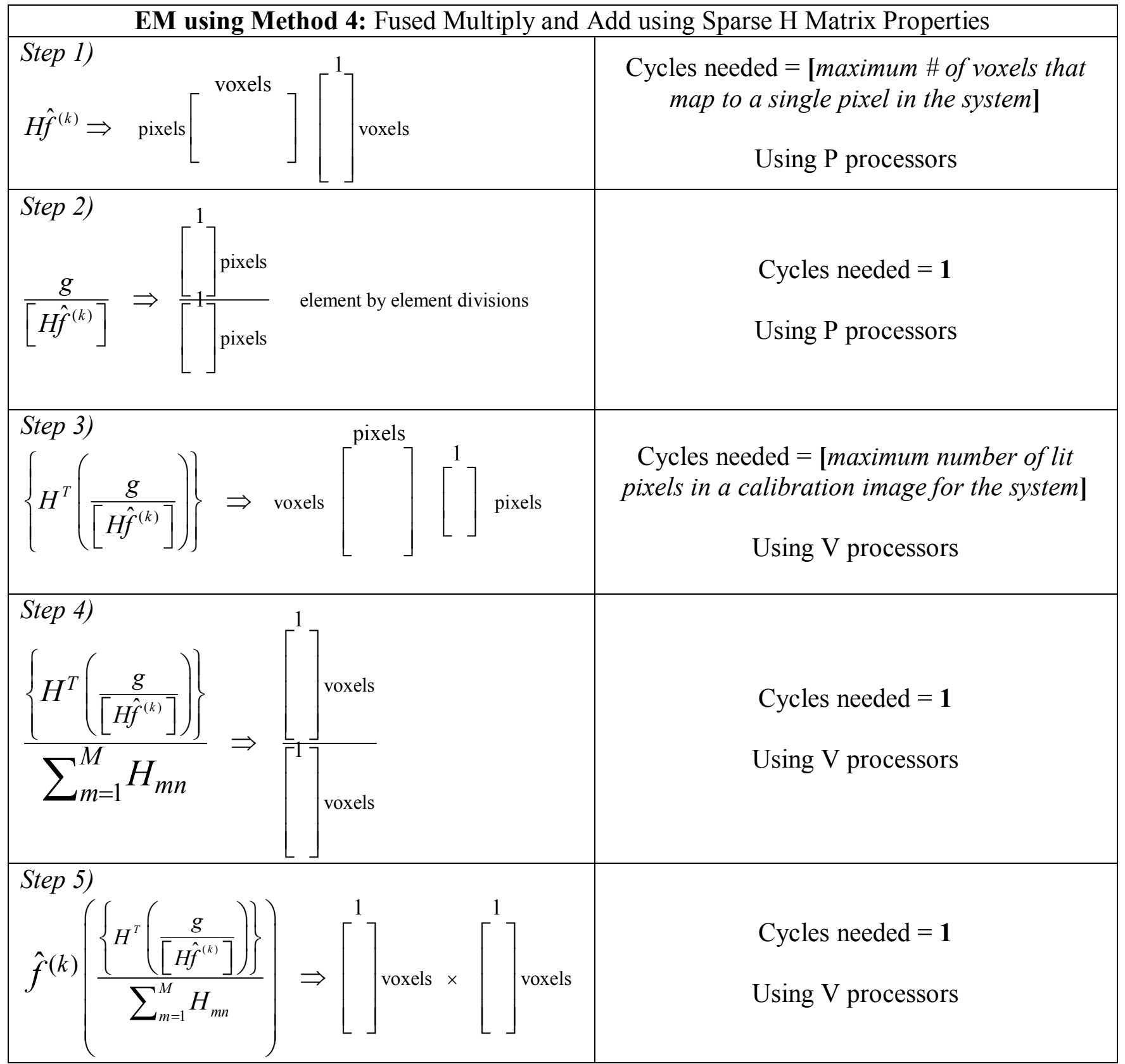

$\mathrm{p}=\#$ of focal plane pixels and $\mathrm{v}=\#$ of datacube voxels. Note that pixels that are never lit by any voxel are not counted in the $\mathrm{p}$ value.

Total Number of Cycles Needed Per Iteration of EM $=\mathbf{3}+[$ maximum \# of voxels that map to a single pixel in the system $]+[$ maximum number of lit pixels in a given calibration image for the system]

Total Processors Needed $=\mathbf{V}$ or $\mathbf{P}$, whichever is greater 


\subsection{CTIS General Case for a single iteration of EM}

\section{CTIS General Case:}

\section{Comparing parallel methods for calculating a single iteration of EM}

1) Parallel Processing of Matrix Vector multiplication using separate multiplication and addition operations

Total \# of processors needed $=($ Total $\#$ of $\mathrm{H}$ matrix elements $)=($ voxels $\mathrm{x}$ pixels $)$

Total \# of cycles $=\mathbf{5}+\mathbf{T}+\mathbf{U}$

Where $T$ is the lowest value integer such that $2^{\mathrm{T}}>(\mathrm{v}-1)$ and $\mathrm{U}$ is the lowest value integer such that $2^{\mathrm{U}}>(\mathrm{p}-1)$

2) Parallel Processing of Matrix Vector multiplication using separate multiplication and addition operations taking into account sparse nature of the matrix

Total \# of processors needed $=(\#$ of nonzero $\mathrm{H}$ matrix elements $)$

Total \# of cycles $=\mathbf{5}+\mathbf{R}+\mathbf{S}$

Where $\mathrm{w}$ is the maximum number of voxels that map to a single pixel in the system and $\mathrm{R}$ is the lowest value integer such that $2^{\mathrm{R}}>(\mathrm{w}-1)$. Note that $\mathrm{R}$ will be less than or equal to $\mathrm{T}$ and that this value may be dramatically decreased by excluding the zero order in the reconstruction calculations. And where $\mathrm{L}$ is the maximum number of lit pixels in a given calibration image for the system and $\mathrm{S}$ is the lowest value integer such that $2^{\mathrm{S}}>(\mathrm{L}-1)$. Note that $\mathrm{L}$ will be less than or equal $\mathrm{U}$.

3) Parallel Processing of Matrix Vector multiplication using a fused multiply and add operation

Total \# of processors needed $=\mathrm{V}$ or $\mathrm{P}$, whichever is larger

Total \# of cycles $=\mathbf{3}+\mathbf{V}+\mathbf{P}$

4) Parallel Processing of Matrix Vector multiplication using a fused multiply and add operation taking into account sparse nature of the matrix

Total \# of processors needed $=(\#$ of voxels or the \# of fpa pixels that can possibly be lit by the CTIS pattern, whichever is larger)

Total \# of cycles $=\mathbf{3}+[$ maximum \# of voxels that map to a single pixel in the system $]+$ [maximum number of lit pixels in a given calibration image for the system]

Note that the maximum number of voxels that map to a single pixel in the system can be often be reduced by excluding the zero order in the reconstruction calculations. 


\title{
7.21 CTIS Example Case for a single iteration of EM
}

\author{
CTIS Example Case: \\ Comparing parallel methods for calculating a single iteration of EM
}

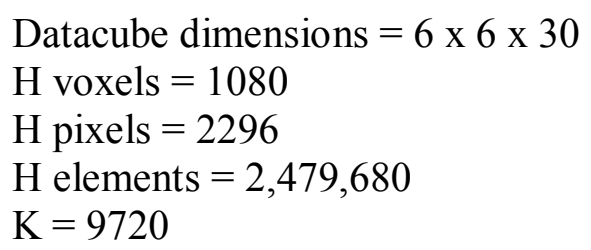

\author{
Method 1) \\ \# of cycles needed $\quad=\underline{\mathbf{2 8} \text { cycles }} \quad(5+11+12=28)$ \\ \# of processors $\quad=2,479,680$
}

\section{Method 2)}

$\{\mathbf{2 A}\}$ If we include the zero order in the data used to determine the reconstruction, then the maximum number of voxels that map to a single pixel in the system is equal to the number of wavelength bands in the system, 30 . Since the example system has 9 diffraction orders, the maximum number of lit pixels per calibration image is 9 .

\# of cycles needed $\quad \underline{\mathbf{1 4} \text { cycles }} \quad(5+5+4=14)$

\# of processors $\quad=9,720$

\{2B When excluding the zero order the maximum number of voxels that map to a single pixel in this simple example system is going to equal the maximum number of spatial resolution elements in one dimension of the zero order image, which in this case is 6 . Note that in more complex systems and systems with diffraction orders that do not have perfect pixel registration (such as a $5 \times 5$ diffraction pattern) this number will be greater. In this case the maximum number of lit pixels per calibration image is now 8 .

\# of cycles needed $\quad \underline{\mathbf{1 1} \text { cycles }} \quad(5+3+3=8)$

\# of processors $\quad=9,720$

Method 3)

\# of cycles needed $\quad=\underline{\mathbf{3 , 3 7 9} \text { cycles }} \quad(3+1080+2296=3379)$

\# of processors $\quad=2,296$

\section{Method 4)}

\{4A\} If we include the zero order in the data used to determine the reconstruction, then the maximum number of voxels that map to a single pixel in the system is equal to the number of wavelength bands in the system, 30 . Since the example system has 9 diffraction orders, the maximum number of lit pixels per calibration image is 9 .

\# of cycles needed $\quad=\underline{\mathbf{4 2} \text { cycles }} \quad(3+30+9=42)$

\# of processors $\quad=2,296$

\{4B When excluding the zero order the maximum number of voxels that map to a single pixel in this simple example system is going to equal the maximum number of spatial resolution elements in one dimension of the zero order image, which in this case is 6 . Note that in more complex systems and systems with diffraction orders that do not have perfect pixel registration (such as a $5 \times 5$ diffraction pattern) this number will be greater. In this case the maximum number of lit pixels per calibration image is now 8 .

\# of cycles needed $\quad=\underline{\mathbf{1 7} \text { cycles }} \quad(3+6+8=17)$

\# of processors $\quad=2,296$ 


\subsection{CTIS General Case for a direct left inverse reconstruction}

\section{CTIS General Case:}

\section{Comparing parallel methods for calculating a direct left inverse reconstruction}

1) Parallel Processing of Matrix Vector multiplication using separate multiplication and addition operations

Total $\#$ of processors needed $=($ Total $\#$ of $\mathrm{H}$ matrix elements $)=($ voxels $\mathrm{x}$ pixels $)$

Total \# of cycles $=\mathbf{1}+\mathbf{C}$

Where $\mathrm{C}$ is the lowest value integer such that $2^{\mathrm{C}}>(\mathrm{p}-1)$

2) Parallel Processing of Matrix Vector multiplication using separate multiplication and addition operations taking into account sparse nature of the matrix

Total $\#$ of processors needed $=(\#$ of nonzero elements in $\mathrm{H}$ left inverse $)$

Total \# of cycles $=\mathbf{1}+\mathbf{D}$

Where $\mathrm{Z}$ is the largest number of nonzero elements in any of the rows of the $\mathrm{H}$ left inverse matrix and $D$ is the lowest value integer such that $2^{\mathrm{D}}>(Z-1)$

3) Parallel Processing of Matrix Vector multiplication using a fused multiply and add operation

Total \# of processors needed $=(\#$ of voxels $)$

Total \# of cycles $=(\#$ of pixels $)$

4) Parallel Processing of Matrix Vector multiplication using a fused multiply and add operation taking into account sparse nature of the matrix

Total \# of processors needed $=(\#$ of voxels $)$

Total \# of cycles $=\mathbf{Z}$

Where $\mathrm{Z}$ is the largest number of nonzero elements in any of the rows of the $\mathrm{H}$ left inverse matrix 


\subsection{CTIS Example Case for a direct left inverse reconstruction}

\section{CTIS Example Case: \\ Comparing parallel methods for calculating a direct left inverse reconstruction}

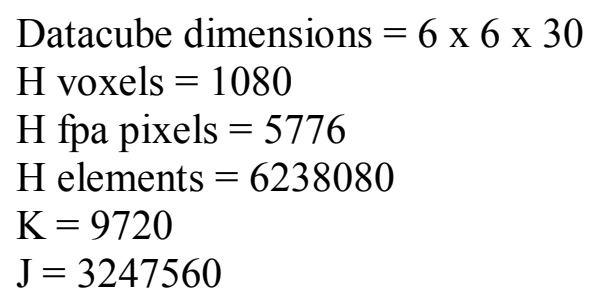

$\begin{array}{ll}\begin{array}{l}\text { Method 1) } \\ \text { \# of cycles needed } \\ \text { \# of processors }\end{array} & =\underline{\mathbf{1 4} \text { cycles }} \\ \begin{array}{l}\text { Method 2) } \\ \text { \# of cycles needed }\end{array} & \leq \underline{\mathbf{1 4} \text { cycles }} \\ \text { \# of processors } & =3,247,560 \\ & \\ \begin{array}{l}\text { Method 3) } \\ \text { \# of cycles needed }\end{array} & =\underline{\mathbf{5 , 7 7 6} \text { cycles }} \\ \text { \# of processors } & =1,080 \\ \begin{array}{l}\text { Method 4) } \\ \text { \# of cycles needed }\end{array} & \leq \underline{\mathbf{5 , 7 7 6} \text { cycles }} \\ \text { \# of processors } & =1,080\end{array}$

\subsection{Parallel processing significantly increases reconstruction speeds}

This analysis shows that implementing parallel processing methods to the reconstruction calculations can produce a dramatic increase in the speed of CTIS reconstructions. In the example CTIS system, the parallel processing methods examined were over 10 times to 3,000 times faster than using sequential operations when completing a single iteration of Expectation Maximization (EM). These same methods were up to 400,000 times faster when using a direct left inverse reconstruction with the example system. (See summary below).

EM sequential required $4 \mathrm{k}+\mathrm{v}=39,960$ operations/cycles

EM parallel required a maximum of 3,379 cycles. (Over 10 times faster.)

EM parallel required a minimum of at least 11 cycles. (Over 3,000 times faster.)

Direct left inverse sequential required $(2 j-v)=6,494,040$ operations/cycles

Direct left inverse parallel required a minimum of at least 14 cycles. (Over 400,000 times faster.)

It is also important to note that this analysis only looked at four methods of parallel processing. There are other methods of parallel processing, not examined in this dissertation, which might further improve reconstruction speed. 


\subsection{Limits on Speed and Upcoming Processing Hardware}

These results for the example CTIS system can be used to estimate how quickly reconstructions can be calculated using parallel processing on certain types of computer hardware. So how fast can it be? Consider the previous example CTIS system and the number of processing cycles needed for some of the reconstruction methods. Each method had a different requirement for the number of processing cores needed to achieve the maximum parallel processing speed. In this analysis, let's assume that the maximum number of required processing cores are available, but each processor is limited to a speed of $1000 \mathrm{MHz}$. Table 5 provides a comparison of the various speeds, illustrating the time needed for each reconstruction as well as the number of possible reconstructions that could be calculated per second.

\begin{tabular}{|c|c|c|}
\hline \multicolumn{3}{|c|}{$\begin{array}{c}\text { With every processor operating at } 1000 \mathrm{MHz} \\
\text { And using the needed number of processors }\end{array}$} \\
\hline $\begin{array}{c}\text { \# of cycles needed } \\
\text { per reconstruction }\end{array}$ & $\begin{array}{c}\text { Time } \\
\text { (in sec) }\end{array}$ & $\begin{array}{c}\text { Reconstructions } \\
\text { per second }\end{array}$ \\
\hline 11 & $1.10 \mathrm{E}-08$ & $90,909,091$ \\
\hline 14 & $1.40 \mathrm{E}-08$ & $71,428,571$ \\
\hline 17 & $1.70 \mathrm{E}-08$ & $58,823,529$ \\
\hline 29 & $2.90 \mathrm{E}-08$ & $34,482,759$ \\
\hline 42 & $4.20 \mathrm{E}-08$ & $23,809,524$ \\
\hline 5776 & $5.78 \mathrm{E}-06$ & 173,130 \\
\hline
\end{tabular}

Table 5: Chart illustrating possible reconstruction speeds for the example CTIS system using N processing cores at $1000 \mathrm{MHz}$, where $\mathrm{N}$ is the number of required cores to achieve maximum parallel processing speed for each reconstruction method.

Note that this is a very simplified analysis, merely comparing the number of processor cores, their processing speeds, and the number of processing cycles needed for a given reconstruction technique. Actual hardware systems will likely have many other factors, such as limited access speed and amount RAM memory, that will become bottlenecks to the overall speed of reconstruction, but the table should provide a best case scenario comparison of reconstruction speeds implying that calculating thousands or even millions of CTIS reconstructions per second could be possible.

New computer hardware is becoming available ${ }^{23,24}$ that provides a huge increase in the number of available parallel processing cores. As previously noted, there are upcoming graphics processors from NVIDIA that will contain 512 processing cores per GPU. By using multiple GPU's together the parallel processing of CTIS reconstructions can be made significantly faster. Unfortunately such parallel processing CPU and GPU hardware was not available for implementation during this work. In the future the parallel methods described can be implemented, but in the meantime we can use our analysis of reconstruction methods to look for ways to modify existing CTIS systems to improve the speed of reconstructions on our existing current computer hardware. Some of these possible modifications are described in the following pages. 


\subsection{Modifications to Increase the Speed of Reconstructions for CTIS Systems}

Without changing existing CTIS systems, calibration techniques, or reconstruction methods, the fastest way to obtain reconstructed data is to implement parallel processing techniques like those outlined in the previous section. However, for the work in this dissertation the computer hardware needed to implement these parallel approaches was not available. Instead of having multiple thousands or even millions of processors to perform the calculations, the reconstructions had to be computed on existing consumer level computer hardware that is far more limited in its ability to perform parallel computations.

The goal now becomes to optimize the sequential operations in such a way as to increase the speed of reconstructions. This method of thinking is what led to the investigation of the direct left inverse technique as a means of performing reconstructions, investigating newly proposed CTIS system designs, and the development of a new reconstruction technique that slightly modifies both the calibration and reconstruction of CTIS systems called the "dirac delta technique." These approaches were developed and demonstrated with the goal of achieving a CTIS that can perform spectral/spatial reconstructions in real time. Lets first examine the direct left inverse reconstruction technique.

\subsection{Direct Left Inverse Reconstructions using Modified CTIS Systems}

The previous chapter described that for certain simple/low resolution CTIS systems the left inverse of the system $\mathrm{H}$ matrix exists and can be used to calculate reconstructions in a single step (no iterations). However, constructed CTIS systems often have much larger spatial and spectral resolutions with significantly larger $\mathrm{H}$ matrices making the left inverse calculation intractable. In order to take advantage of the direct left inverse technique and its corresponding reconstruction speed for low res systems, CTIS systems would need to be modified to effectively reduce the resolution of the system such that the left inverse calculations were more practical.

One way to implement this on an actual CTIS system would be to physically decrease the size of the field stop to limit the spatial resolution and add a narrow spectral bandpass filter to decrease the spectral resolution. The smaller field stop and narrower spectral band would now only use a small fraction of the focal plane compared to the full resolution system. The remaining part of the FPA would be black. Yet another way of accomplishing the same effect would be to only view a single small object on a black background. In such a case, a CTIS system should be capable of producing a direct inverse reconstruction (no estimation) of that object as long as its spatial extent of the object is 6x6 pixels or less (using only information from 9 diffraction orders in a $3 \times 3$ pattern). The black background ensures that there will be no overlap of spectral data from spatial locations outside the 6x6 pixel region of interest in the same manner as using smaller sized field stop. A direct left inverse reconstruction could be done using just the data from the selected regions of interest.

A demonstration of a direct left inverse reconstruction, and its corresponding reconstruction speed, is presented below using raw data taken from a visible CTIS system. In this demonstration, the image to be reconstructed is a raw calibration image from a visible CTIS 
system shown in figure 7.7. The image consists of a spatially point like object with a fairly narrow spectral bandwidth of about $8 \mathrm{~nm}$, centered at $680 \mathrm{~nm}$. The object's spectral intensity distribution should be like that of a triangle function since the monochromator slit widths used to produce the monochromatic light were equal in width. (The spectral output of the monochromator is related to the convolution of the entrance slit with the exit slit, with the convolution of a rect function with an equal sized rect function producing a triangle function.) Even though the size of the zero order of the full sized CTIS system was $100 \times 100$ pixels, since the object was located on a black background, one could construct an equivalent $\mathrm{H}$ matrix for the $6 \times 6$ pixels that enclosed the object in the zero order that also only uses diffraction order data from 9 of the outermost diffraction orders. Note that the constructed $\mathrm{H}$ matrix was also limited to 40 spectral bands. The results from this test, which are shown in figure 7.8 , demonstrate that using these limitations/modifications reconstructions on an existing CTIS can now be done at 21 frames per second on current computer hardware!

This single addition to the CTIS would bring the added functionality of quick reconstructions to the system. Having the ability to perform real time reconstructions with the systems would be a great advantage and would allow for the real time detection and identification of objects/targets in the field based on their unique spectral and polarization signatures.

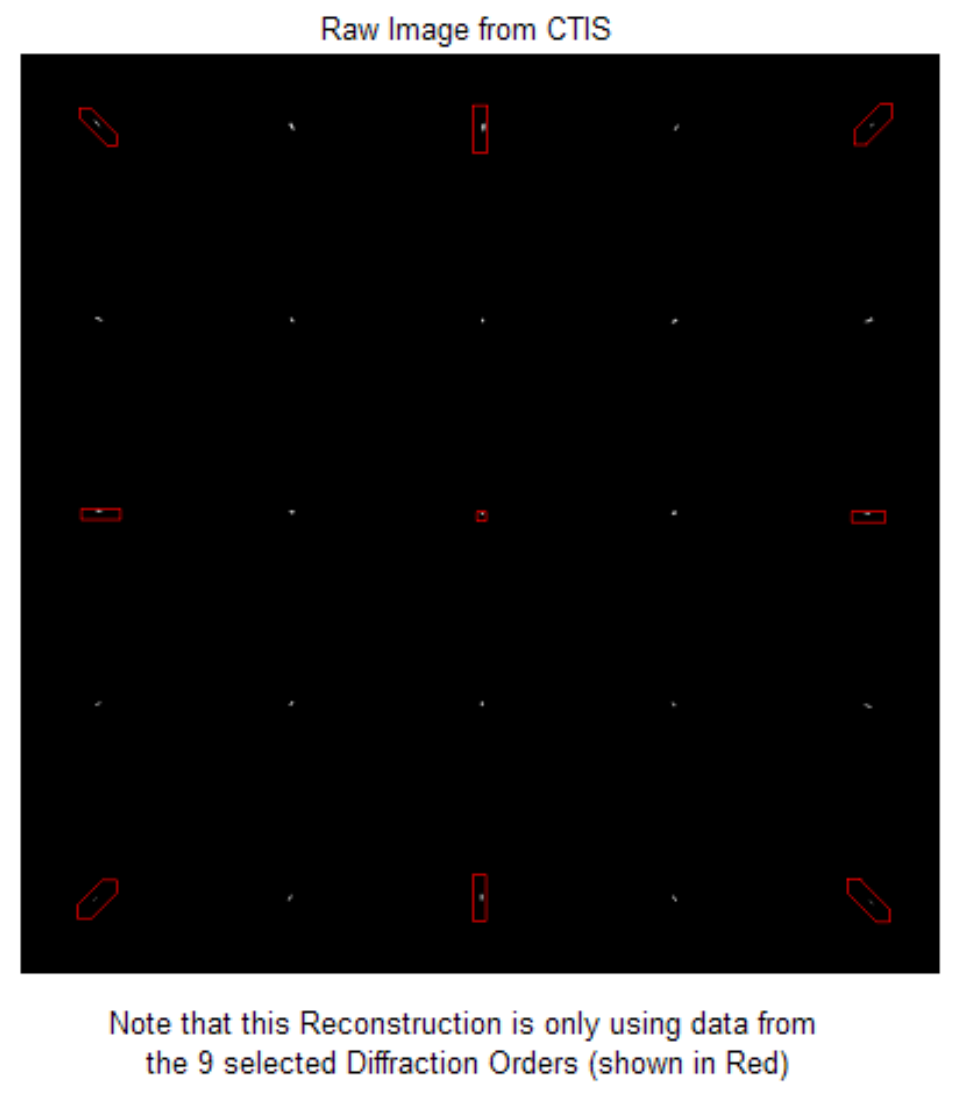

Figure 7.7: Raw calibration image from visible CTIS system. Pixel data within the 9 highlighted regions will be used to demonstrate a direct left inverse reconstruction. 


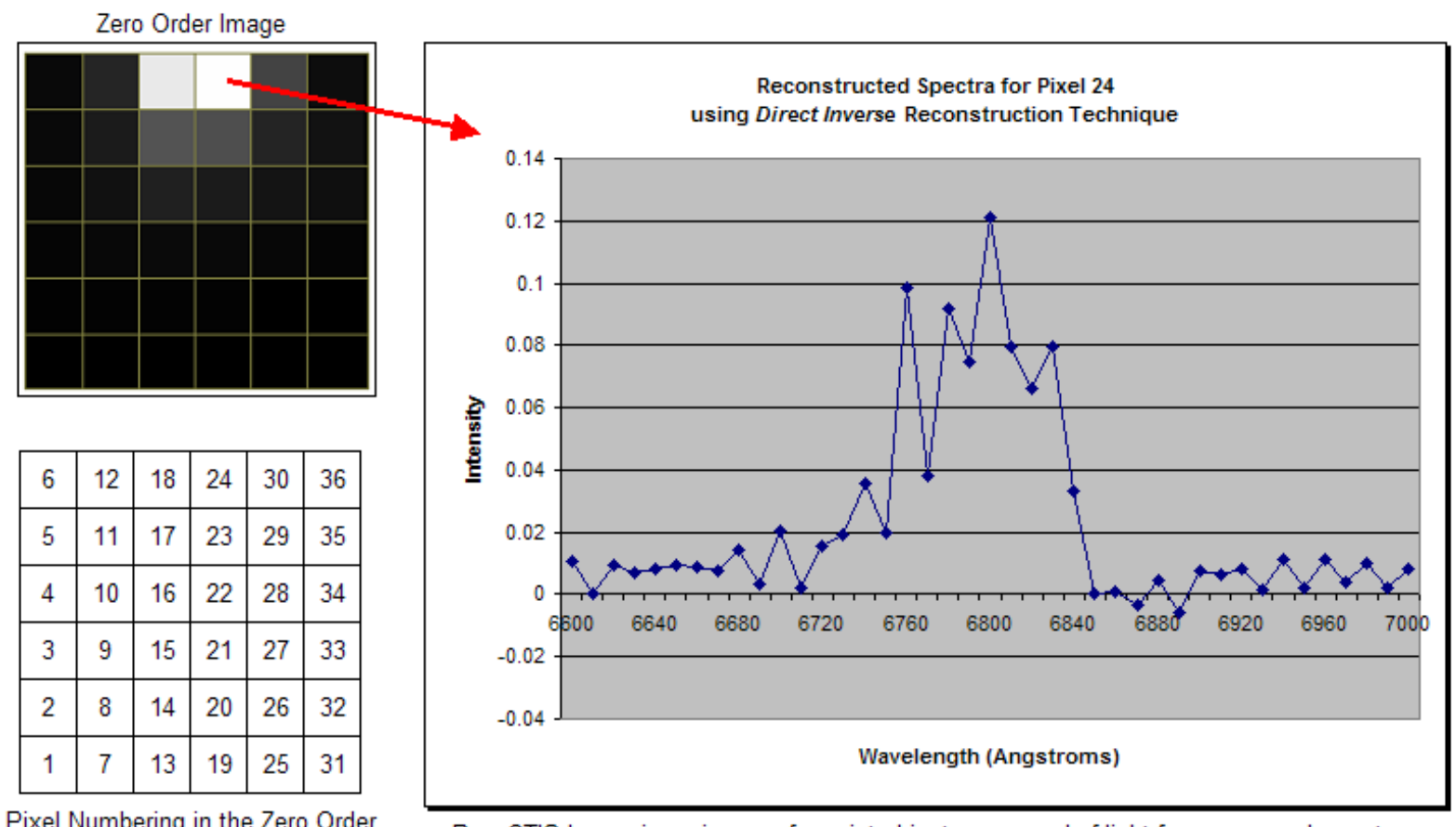

Pixel Numbering in the Zero Order

Raw CTIS Image is an image of a point object composed of light from a monochrometer centered at $680 \mathrm{~nm}$ with a FWHM spectral bandwidth of approximately $8 \mathrm{~nm}$

\section{Reconstruction Time for $6 \times 6$ data cube $w / 40$ spectral bands $=0.047$ seconds can reconstruct 21 frames per second}

Figure 7.8: Demonstration of Direct Left Inverse reconstruction on actual CTIS data

The previous example looked at a case where the objects in the scene were set against a black background, however for many hyperspectral imaging applications the objects of interest will not necessarily be against such a black background. For these more general purpose applications the CTIS system must be modified in order to incorporate the left inverse technique and take advantage the technique's fast single step reconstructions. One way to incorporate the left inverse reconstruction technique would be to add a spatial light modulator or digital micromirror array at the field stop of the CTIS system. The SLM or micromirror array acts like an active variable size field stop, allowing the operator to effectively turn off or block all the light from the scene except for a particular 6x6 pixel spatial region in the zero order. This would be equivalent to having a black background across the entire scene, except for the selected lit spatial region of interest. Since the operation of the SLM or micromirrors can be done extremely quickly, a high resolution image of the scene could be obtained by changing the position of the $6 \times 6$ spatial region for each captured frame and combining the reconstructed data from a series of frames.

Another approach would be to use pixel binning to artificially lower the resolution of the FPA. Pixel binning combines a set of adjacent camera pixels into a single signal output. For example a two by two square of 4 pixels on a CCD chip might be reported as one pixel containing their combined value. For an existing CTIS system, one could bin certain groups of pixels from the zero order to create an effective $6 \times 6$ sized zero order. The same type of binning could also be used for pixels in the diffraction orders as well. 
Incorporating both of these methods into a single CTIS design would provide an extreme amount of control for the resolution and location of the selected region of interest to be measured using the direct left inverse reconstruction. Because the reconstruction processing could be done in real time, these different combinations could be quickly cycled or changed as the instrument is in operation based on the analyzed reconstructed data from each frame. For example, by utilizing a database of existing $\mathrm{H}$ matrices for the system, the CTIS could quickly identify a general region of interest in the scene and then acquire a higher resolution set of data for that particular region of interest. Subsequently the system could use that new reconstructed data to better define a smaller region of interest over the target, and then acquire an even higher spatial and spectral resolution dataset just for the target. This could all be done in just a handful of frames. A more detailed example of this type of operation for this newly proposed CTIS design is illustrated in the following diagrams.

\subsection{Sample Operation of Modified CTIS using Left Inverse Reconstruction}

For this example CTIS system, the zero order has a size of 48 pixels by 48 pixels on the FPA. An appropriately sized spatial light modulator is placed at the field stop of the system allowing the light hitting each pixel in the zero order to be either blocked (absorbed by the SLM) or passed through to the rest of the CTIS system. The full resolution of the system is shown in figure 7.9 .

Step 1: For the first recorded frame of data, the CTIS system will set the SLM to be completely transmissive and will bin pixels on the CCD output using groups of $8 \times 8$ pixels. This binning level has been selected to allow the zero order to have a resolution of $6 \times 6$ effective pixels, allowing the use of the single step left inverse reconstruction technique. This recorded frame will be analyzed using the left inverse reconstruction technique with an $\mathrm{H}$ matrix covering the entire spectral region of the CTIS system. Note that this H matrix may have a lower spectral resolution due to binning of pixels in the outer orders of the FPA image.
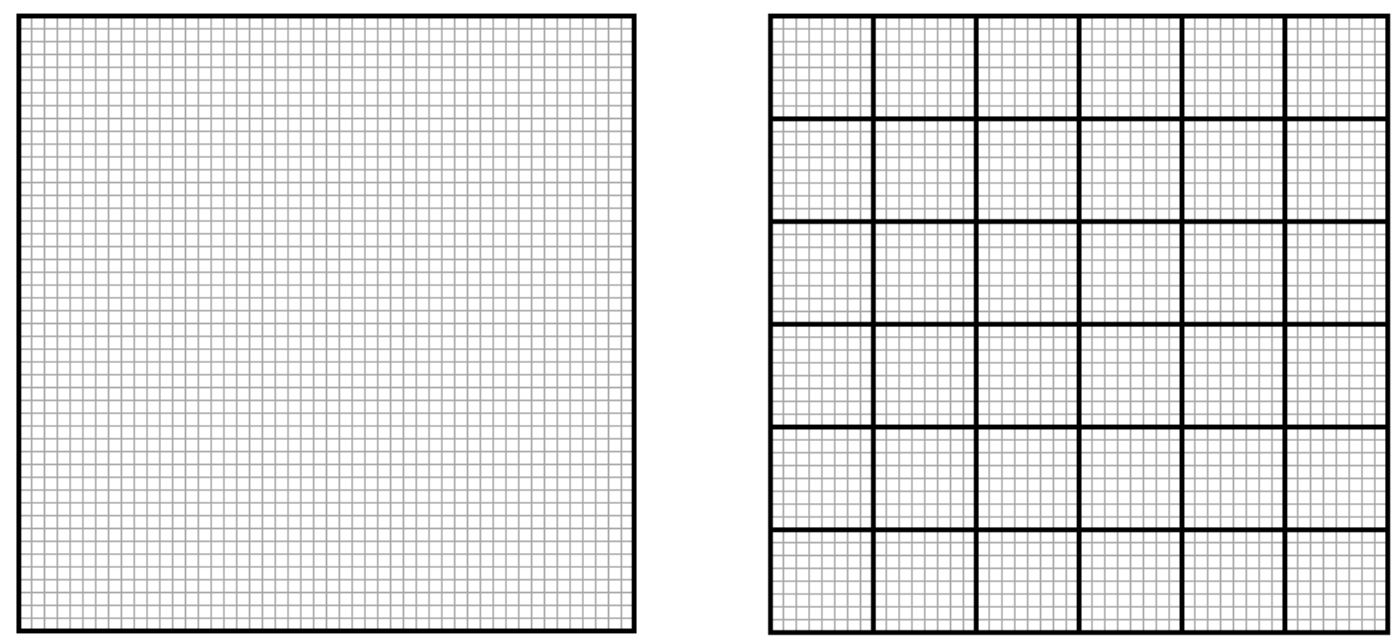

Figure 7.9: (Left) Illustration of the full resolution 48 by 48 pixel resolution of the CTIS system's zero order. (Right) Zero order illustration showing the $8 \times 8$ binning of individual pixels and the $6 \times 6$ effective resolution of the zero order after binning. 
Step 2: Detect object of interest using the reconstructed spatial and spectral data from the first frame. Figure 7.10 shows the reconstructed zero order for this frame. An object of interest is detected based on the reconstructed spatial and spectral data and is highlighted in orange in figure 7.10 .

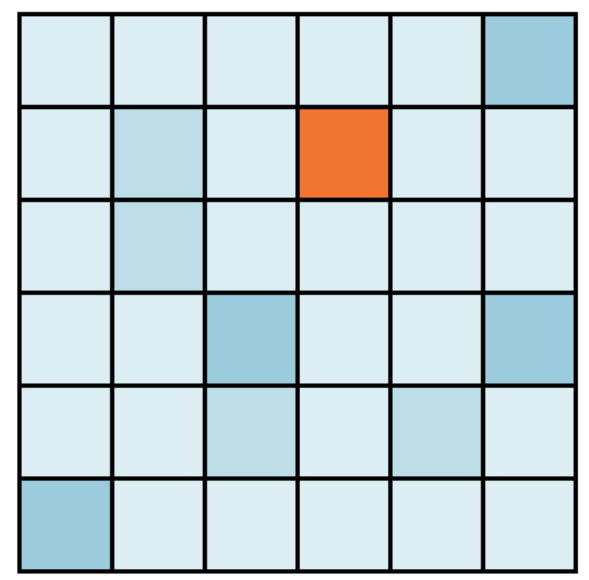

Figure 7.10: Recorded Zero order image for first frame of data.

Step 3: Use the spatial light modulator to turn OFF or block light from the entire scene except for the detected region of interest, as illustrated in figure 7.11. This smaller area will be defined as the zero order for the following frame. The binning of the FPA is changed to now have a binning of $2 \times 2$ pixels in order to decrease the spatial resolution enough to use the left inverse reconstruction technique for this section of the FPA. These new binning settings will provide an improved spatial resolution for this area compared to the data from the first frame. The spectral region of interest will also be redefined based on the previously recorded data in an attempt to get finer details of spectral features, and the corresponding $\mathrm{H}$ matrix for these settings is pulled up from a database. A second frame of data is then acquired by the system using these new modulator and binning settings. The zero order for this second frame of data is shown in figure 7.12 .

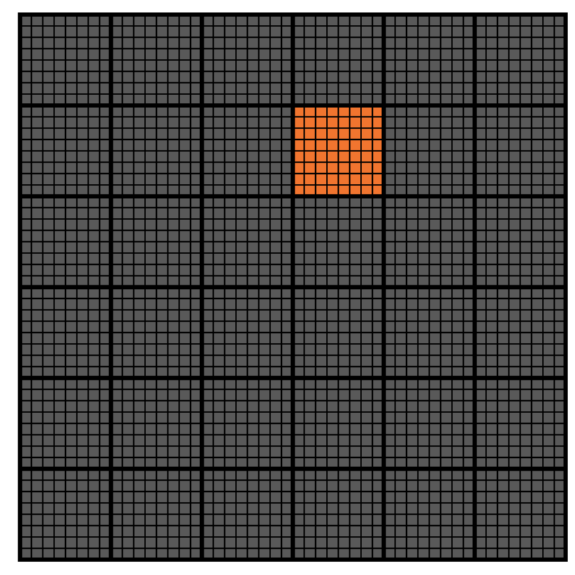

Figure 7.11: The spatial light modulator is used to block light for all of the physical pixels of the FPA except for the region of interest defined by the reconstructed data from the first frame. 


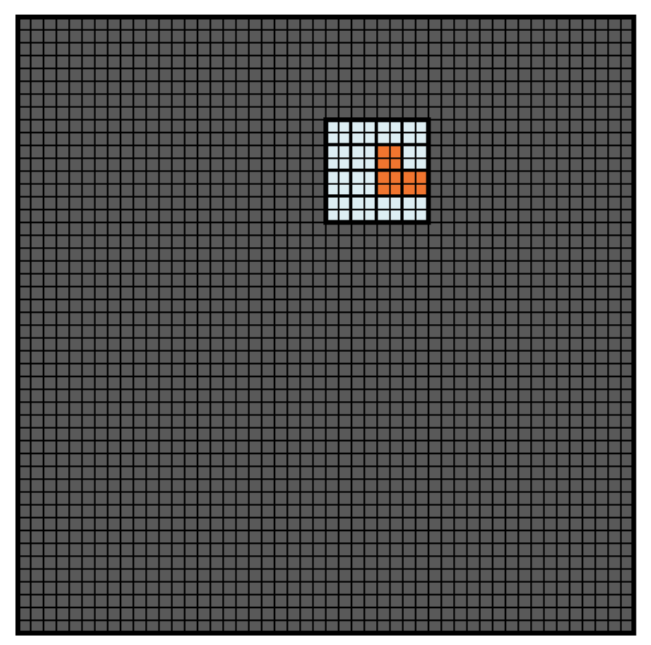

Figure 7.12: Recorded Zero order image for the second frame of data. Note that the spatial light modulator was used to block light for from a majority of the system's field of view and that the remaining pixels in the narrower field of view utilize a $2 \times 2$ pixel binning.

Step 4: Reconstruct the spatial and spectral data from frame 2 using the direct left inverse technique and use this reconstructed information to better define or characterize the target's location, shape, and spectral features. Figure 7.12 now shows a slightly higher spatial resolution image of the region of interest, and the target highlighted in orange, compared to what was acquired in figure 7.10 .

Step 5: Repeat steps 2 through 4. Define a new region of interest, turn OFF light from all spatial positions outside of your target, and if necessary change the binning output from the FPA. Acquire the next frame. Load the appropriate $\mathrm{H}$ matrix for these settings and then reconstruct the spatial and spectral data for that frame. Use the reconstructed spectral data to define a new spatial and spectral region of interest, taking into account the zero order size and possible FPA binning, and repeat the process. The highest spatial resolution will be achieved when the modulator blocks all light from the zero order except for a $6 \times 6$ region of physical pixels with no binning, like the case illustrated in figure 7.13 . 


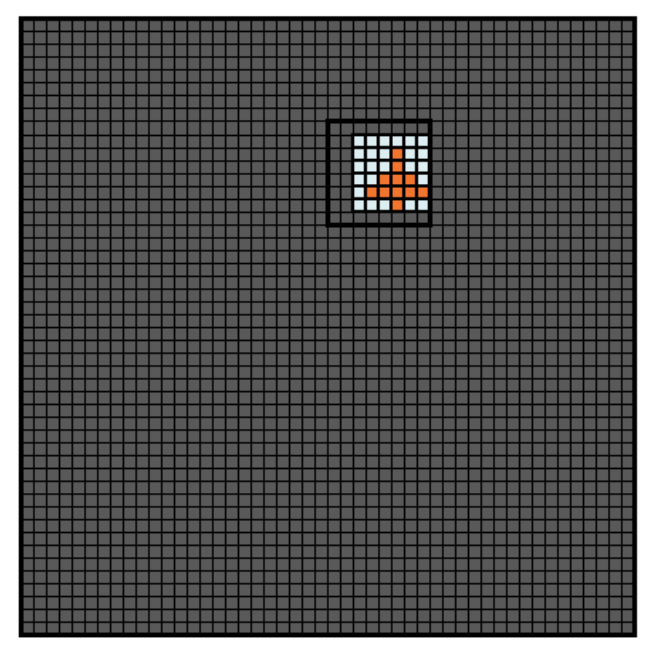

Figure 7.13: Recorded Zero order image for a subsequent frame of data. Note that the only portion of the zero order not blocked by spatial light modulator is a $6 \times 6$ group of pixels on the FPA with no binning. This corresponds to the highest spatial resolution achieved by this modified CTIS system utilizing the direct left inverse technique.

This modification to the CTIS takes advantage of the fast reconstruction processing of the direct left inverse technique, which has been currently demonstrated at 21 frames per second on commercial computer hardware, to allow the system to rapidly acquire high resolution hyperspectral data for an object or group of objects in the scene. Note that the acquisition of high resolution data for the entire scene is no longer snapshot, but can be acquired in a limited number of frames through a rapid succession of different SLM and binning combinations. This approach will help bring the system closer to the goal of real time operation, but the addition of extra hardware and processing routines can add significantly more complexity to the CTIS system. A third approach has been investigated that requires no additional hardware and adds very little complexity to current CTIS systems. It tries to increase the speed of reconstructions by making the system $\mathrm{H}$ matrix as sparse as possible, and is referred to as the dirac delta reconstruction technique.

\subsection{Dirac Delta CTIS Reconstruction Technique}

\section{Trade Spatial Resolution for Increase in Speed}

The dirac delta CTIS reconstruction technique can drastically speed up CTIS reconstructions. It uses the same iterative reconstruction algorithms of EM and MART, can be used on low resolution CTIS systems as well as high resolution systems, and only consists of a slight change to the CTIS calibration method. The technique works by creating a modified $\mathrm{H}$ matrix/set of "synthetic" calibration images that consist of only delta functions as opposed to the PSF's of the optical system, making the resulting system $\mathrm{H}$ matrix extremely sparse which in turn increases reconstruction speed. In effect this reconstruction technique aims to trade a small amount of 
spatial resolution in reconstructed data for an increase in the speed of the reconstruction while keeping the spectral resolution of the system the same. If higher spatial resolution data is required, additional deconvolution post processing steps can then be applied to regain some of the lost spatial resolution at a cost of processing speed.

The idea of using synthetic calibration images to form the $\mathrm{H}$ matrix has been previously used to address low light level issues when recording calibration images, but this is the first use of modified calibration images specifically aimed at maximizing the reconstruction speed of a CTIS system. Before covering the details of the technique and how it compares to conventional reconstructions lets first examine how reconstruction speed is related to the sparseness of the $\mathrm{H}$ matrix and some of the CTIS system parameters.

Recall that the total number of calculations needed to complete a single iteration of Expectation Maximization is $4 \boldsymbol{k}+\boldsymbol{v}$, where $\boldsymbol{k}$ is the number of nonzero elements in the system $\mathrm{H}$ matrix (a measure of how sparse the matrix is) and $\boldsymbol{v}$ is the number of datacube voxels. For sequential operations, the reconstruction will be completed in a shorter amount of time if fewer calculations are required. So decreasing the number of nonzero elements in the system $\mathrm{H}$ matrix will produce sparser CTIS systems H matrices with faster reconstruction speeds.

With that in mind, each the following parameters of the CTIS system and corresponding $\mathrm{H}$ matrix/calibration image set can be modified to decrease the number of nonzero elements in the $\mathrm{H}$ matrix:

1. Number of lit pixels in each calibration image

1.1. Size of the recorded PSF (in \# of pixels) for each diffraction order

1.2. Number of diffraction orders

2. Number of calibration images/datacube voxels

2.1. Number of spatial positions

2.2. Number of wavelength bands

The following sections will describe how modifications to each of these parameters can be incorporated using the dirac delta reconstruction technique and the relative increase in speed associated with each modification.

\section{Size of PSF in pixels}

The dirac delta reconstruction technique achieves an increase in reconstruction speed primarily by addressing the size of the PSF (in pixels) in each calibration image. As its name implies, the dirac delta reconstruction technique will use dirac delta functions to replace the recorded optical PSF's of the system when creating a synthetic calibration image set used to form the system $\mathrm{H}$ matrix. As a result, the number of nonzero elements in the $\mathrm{H}$ matrix will be decreased by a factor related to the size of the PSF's in the system. If the PSF for a CTIS system generally covers 25 pixels ( $5 \times 5$ pixels) in each diffraction order, reconstructions using an $\mathrm{H}$ matrix of delta functions would be done approximately 25 times faster. To put this single change into perspective, CTIS reconstructions that previously would have taken 50 minutes to complete could now be done in 2 minutes! 
Number of diffraction orders

Reducing the number of diffraction orders in the calibration images will reduce the number of lit pixels in each calibration image making the system $\mathrm{H}$ matrix sparser. Even though the physical CTIS system will have a fixed number of orders, for the purposes of reconstruction calculation and the creation of the synthetic calibration images, the user can select which diffraction orders will be used.

This can be especially useful to eliminate the orders containing redundant information in an existing CTIS system to give a moderate boost to the reconstruction speed. These eliminated diffraction orders in the synthetic calibration images will be completely black with pixel values of zero. For example in a CTIS with a full 5x5 diffraction pattern array, if only the outer ring of orders were used for the reconstruction calculation, the reconstruction would be approximately 1.5 times faster. Similarly a $3 \times 3$ pattern (the subset of the outermost diagonal, horizontal, and vertical orders) could be selected from the initial $5 \times 5$ array to provide an approximate 3 times increase in reconstruction speed. Again note that this current analysis is only considering reconstruction speed and not necessarily reconstruction quality.

\section{Number of spatial positions}

The number of spatial positions is generally set by the size of the zero order in pixels. Decreasing the size of the field stop can reduce the number of spatial positions, and thus reduce the number of voxels, allowing for a slightly faster reconstruction but at a cost of reducing the system's field of view. For example decreasing the zero order size from 100x100 pixels to 70x70 pixels should produce reconstructions approximately 2 times faster.

\section{Number of wavelength bands}

The system designer can arbitrarily choose the number of spectral wavelength bands in the system. However, in order to maximize the speed of reconstructions with the dirac delta technique, the designer should make this selection based upon the spectral spread per pixel in the diffraction orders with the highest dispersion and the total amount of dispersion in those orders. Ideally, the selected calibration wavelengths and wavelength bandwidths should cover the entire spectral range at bandwidth steps that correspond to integer pixels steps in the CTIS image in order to minimize the number of lit pixels in each calibration image.

The net effect of incorporating changes to all of these parameters is that reconstruction speeds for a given CTIS system can often be made well over an order of magnitude times faster than the conventional approach while still using the same computer hardware.

\subsection{How the Technique Works}

As previously mentioned, there is a trade off made when using the dirac delta reconstruction technique. You are effectively trading a small amount of spatial resolution in the reconstructed data for an increase in reconstruction speed. To illustrate how this works and the difference 
between it and conventional reconstructions, consider the example of using a physically recorded CTIS calibration image as the input image to be reconstructed and compare the datacube outputs when using an $\mathrm{H}$ matrix composed of PSF calibration images for reconstruction and an $\mathrm{H}$ matrix composed of modified delta function calibration images.

Since the image to be reconstructed is a physically recorded calibration image, its datacube corresponds to a datacube with only a single lit voxel. In other words the object associated with the image is a single point object emitting monochromatic light. When performing the reconstruction, the conventional $\mathrm{H}$ matrix will be composed of PSF calibration images, but the modified $\mathrm{H}$ matrix will be composed of dirac delta calibration images as illustrated in figure 7.14 .
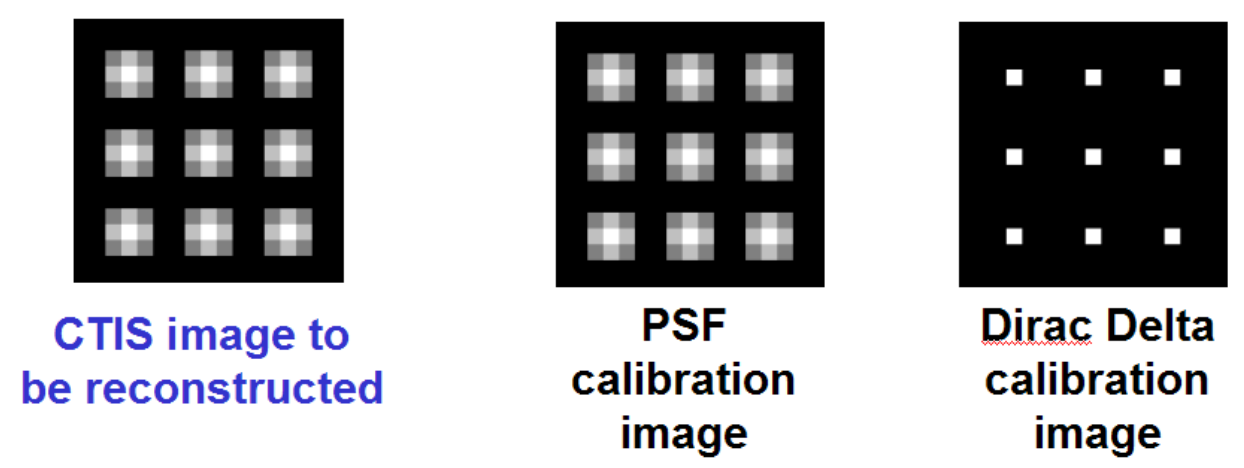

Figure 7.14: Comparison of the image to be reconstructed (left), a calibration image composed of PSF's (center), and a calibration image composed of dirac delta functions (right). 


\section{CTIS Image to be} Reconstructed
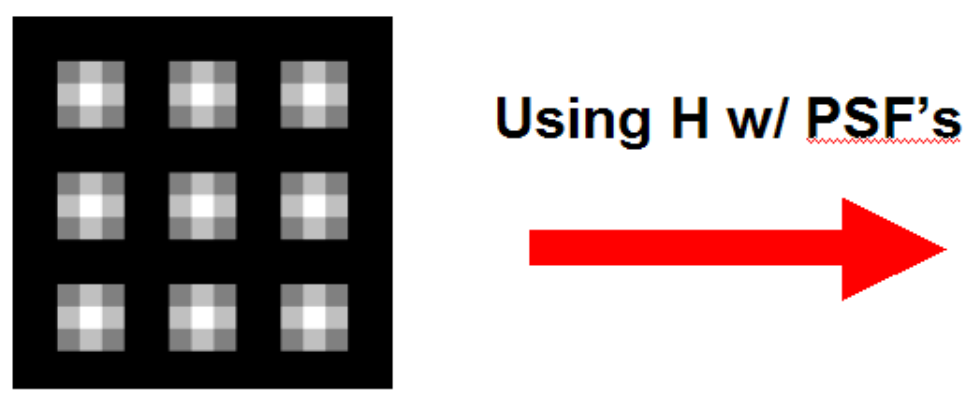

\section{Reconstructed Results}

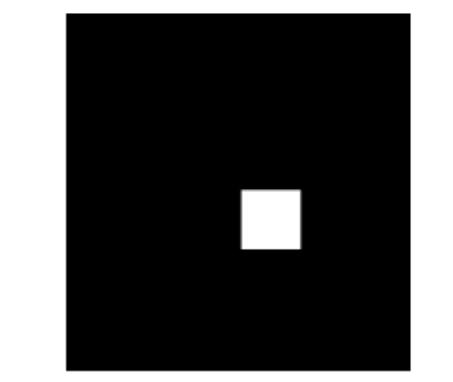

Datacube with single lit voxel

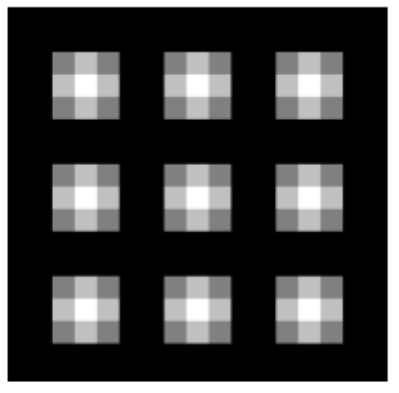

Original CTIS image is a calibration image with only a single lit voxel

\section{Using H w/ Deltas}

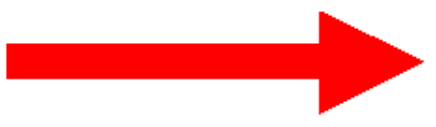

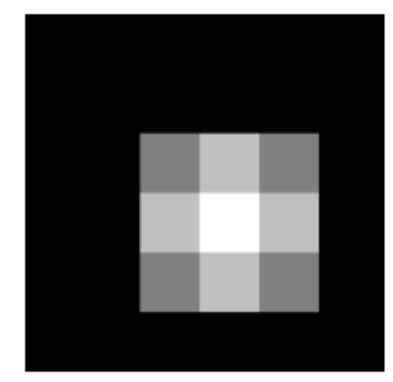

Datacube with multiple lit voxels

Figure 7.15: Comparison of the reconstructed results when using an $\mathrm{H}$ matrix composed of PSF calibration images and an $\mathrm{H}$ matrix composed of dirac delta calibration images. The use of the modified dirac delta calibration images produces a significantly faster reconstruction but has reduced spatial resolution in the reconstructed results.

During the reconstruction, the input image will be compared with the calibration images found in the $\mathrm{H}$ matrix and weights will be assigned to each voxel depending upon how similar or dissimilar each calibration image is with the associated parts of the input image. Since the input image is the exact same as one of the PSF calibration images found in the conventional PSF H matrix, the corresponding single voxel will have a high value in the reconstructed data when using the PSF H matrix, while all other voxels will have low values. However when using the delta function $\mathrm{H}$ matrix, if the PSF shape is the same in all of the diffraction orders as shown in the illustration above, then there will be a single voxel/calibration image that matches each spatial location of the PSF in the recorded image. In the example illustration, there will be a dirac delta calibration image where the location and spacing between the orders of the lit pixels in that calibration image will match the top left corner pixel of all of the PSF's in the input recorded image. There will be a separate calibration image (a shifted version of the previous one with the same wavelength) that will match all its pixels with the top center portion of all the PSF's in the input image, etc. In the resulting reconstructed data there will now be multiple lit voxels, with the irradiance of each voxel scaled to match the relative irradiance of each spatial 
position in the PSF, as shown in figure 7.15. Comparing these two results shows that the reconstructed wavelength is the same for both cases, but the use of the delta function $\mathrm{H}$ matrix has resulted in a slight loss of spatial resolution compared to the conventional approach.

This example shows how the reconstructed results compare when looking at a monochromatic point object, but the same type of results were found for a variety of other objects types: multiple spectral line spatially point like objects, spectrally broadband point like spatial objects, spatially broad objects with broadband spectrums, etc. irregardless of the reconstruction method used (direct left inverse, EM, etc.). The results show that when comparing systems with an $\mathrm{H}$ matrix made of PSF's and systems with a modified $\mathrm{H}$ matrix made of delta functions the spectral resolution should be the same for both the PSF and Dirac Delta H matrices, with the primary difference being that the delta $\mathrm{H}$ matrix reconstructions have a lower spatial resolution in the results. The dirac delta technique allows a single modification to the calibration images to trade spatial resolution in the reconstructed results for an increase in reconstruction speed.

In addition to the previously outlined speed increases for the calculation of each iteration of EM, the dirac delta technique has also been shown in simple CTIS simulations to converge faster to its associated datacube solution when compared to equivalent systems with a conventional PSF $\mathrm{H}$ matrix. That is to say that fewer iterations of EM are needed. The solution from the dirac delta reconstruction will still suffer from the loss of spatial resolution tradeoff, with multiple lit voxels, but on an iteration by iteration basis it will reach that result faster than the conventional PSF system will reach the solution of the single lit voxel. So the modifications made by the dirac delta technique allow each iteration of EM to be calculated faster and requires fewer iterations of EM to approach its solution. In testing, useful reconstructed spectral data can be achieved using only a single iteration of EM. Results of the dirac delta reconstruction technique implemented in actual CTIS systems are shown on the following pages. 


\subsection{Demonstrated Reconstruction Speed Increases Using Dirac Delta Technique}

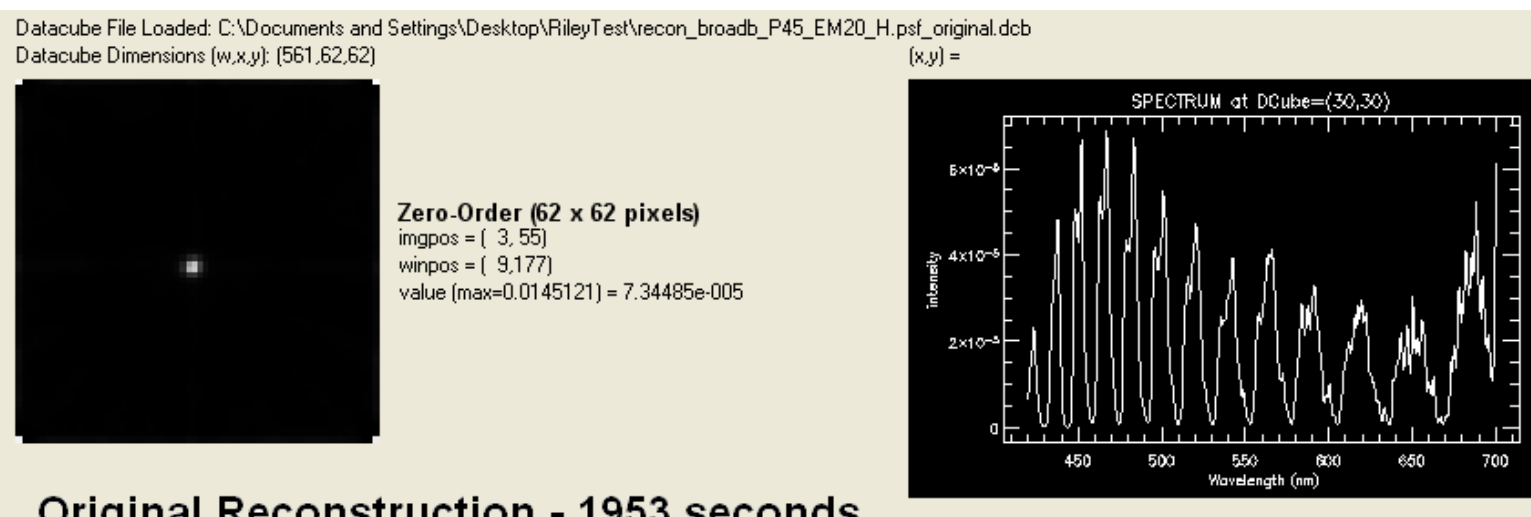

Original Reconstruction - 1953 seconds

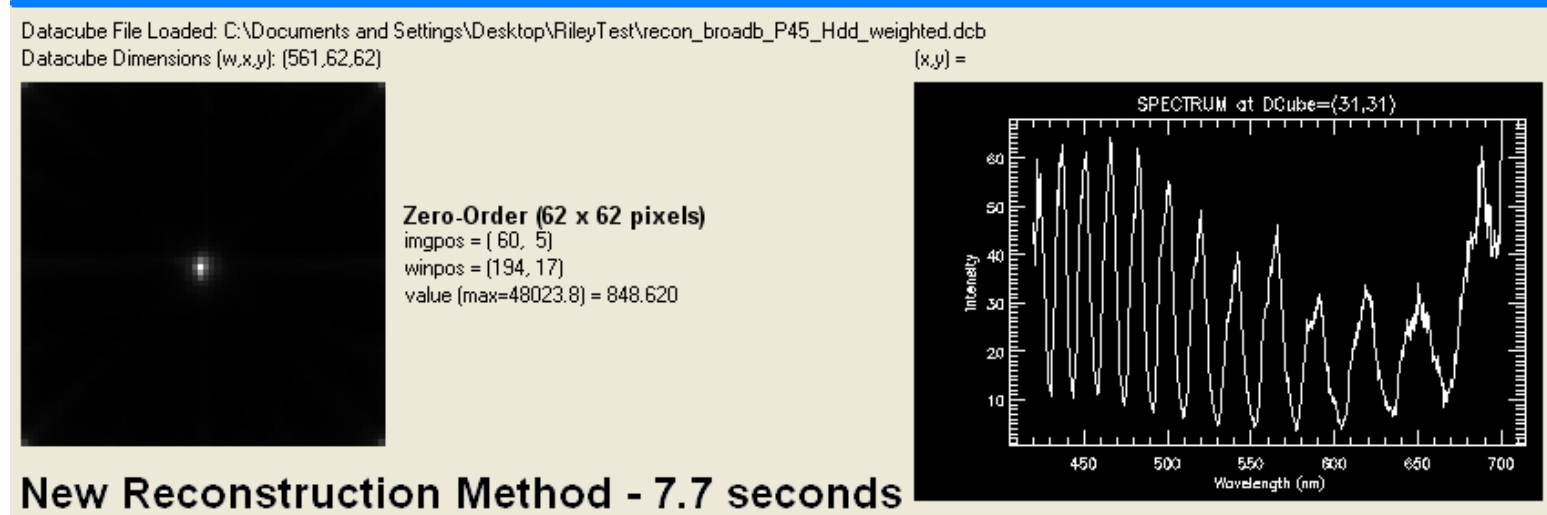

New Reconstruction Method - 7.7 seconds

Zero-Order (62 x 62 pixels)

imgpos $=[60,7]$

winpos $=(195,24)$

value $[\max =11260.4]=918.537$
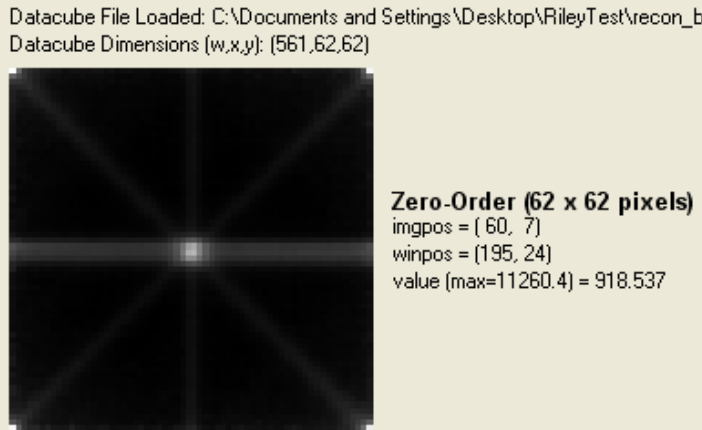

Using Outer $3 \times 3$ Orders -2.47 seconds

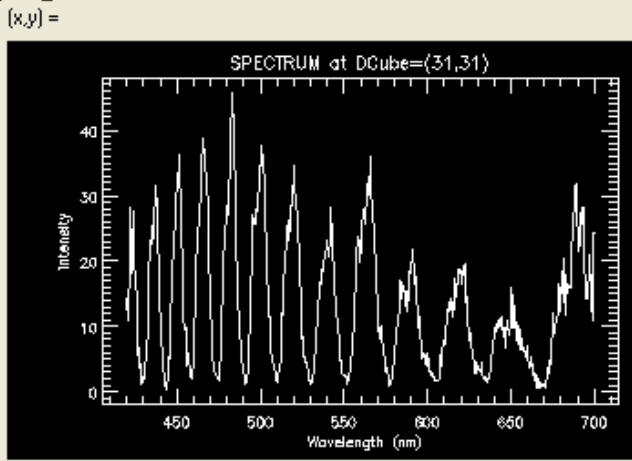

Figure 7.16: Comparison of reconstructions showing the $\mathrm{x} y$ spatial data on the left and spectral data from the center position on the right. (Top) Results using 20 iterations of EM and PSF calibration image set. (Middle) Results using 1 iteration of EM with dirac delta calibration image set. Note each calibration image contains 25 diffraction orders. (Bottom) Results using 1 iteration of EM with dirac delta calibration image set with only 8 diffraction orders. 
The reconstructions in figure 7.16 are of a spectrally broadband point source polarized at 45 degrees that has its spectrum modulated due to channeled spectropolarimeter components inside a visible wavelength CTIS system. Data from the conventional reconstruction method is shown at the top of the figure, while data using the new method is shown in the middle and bottom sections. Note that the conventional method using the PSF H matrix was the result of 20 iterations of EM, while the dirac delta results only used one iteration of EM even though the reconstructed spectral results are markedly similar for both approaches.

The spatial resolution tradeoff can be seen when comparing the reconstructed spatial data of the various methods. In the original method, you see that the broadband point is reconstructed as to a space of about $4 \times 4$ pixels, with the center $2 \times 2$ group of pixels being the brightest. This is a bit smaller in comparison to the new dirac delta method, which reconstructs the same broadband point object to a larger area of about $5 \times 5$ or $6 \times 6$ pixels. The new method, however, has a single bright spot in the center and Gaussian like falloff over the rest of the area. The results show that the spatial resolution of the reconstruction isn't quite as high, but the reconstruction was now done significantly faster needing only a single iteration of EM.

The final set of data, shown at the bottom of figure 7.16, illustrates this effect even further. The speed of the reconstruction can be increased by limiting the number of diffraction orders analyzed in the reconstruction, but at the cost of a decrease in spatial resolution. In this case only 8 orders, the outer ring of a $3 \times 3$ pattern composed of the just the outermost diagonal, horizontal, and vertical orders of the original $5 \times 5$ CTIS pattern (no off diagonal orders), were used to in the reconstruction calculation. You can see that the point object has been reconstructed to a star shape, with each of the lines representing the contribution of the backprojection of each of diffraction orders to the spatial reconstruction. Having more iterations will reduce this effect, making the reconstruction much more point like, but with slower reconstructions. For an application where the operator is primarily concerned about the spectrum of an object and only need its general location, this "outer $3 \times 3$ " method combined with the dirac delta technique could be very useful.

Figures 7.17 through 7.19 on the following page demonstrate the increase in speed of the dirac delta reconstruction technique. In this example the visible CTICS system has a $5 \times 5$ diffraction order pattern and is viewing a white light point source polarized at 45 degrees producing a modulated spectrum. The results show that the same modulated features are present in the dirac delta reconstructed data but the data from the original PSF system is much smoother in comparison to the higher frequency dirac delta data. Depending upon the application, the dirac delta results may be sufficient. Slight tweaks to the implementation of the dirac delta technique can be made to try to reduce this effect and improve these results. For example the irradiance or pixel values of the synthetically generated dirac delta calibration images could be tweaked to control the relative intensity of the reconstructed data. Other factors such as the pixel alignment of the diffraction pattern and the specifics of how the dirac delta calibration images are generated can also cause some of the much higher frequency features in the reconstructed data. Some details on the implementation of the dirac delta reconstruction technique and the formation of the synthetic calibration images are presented in the following pages. 


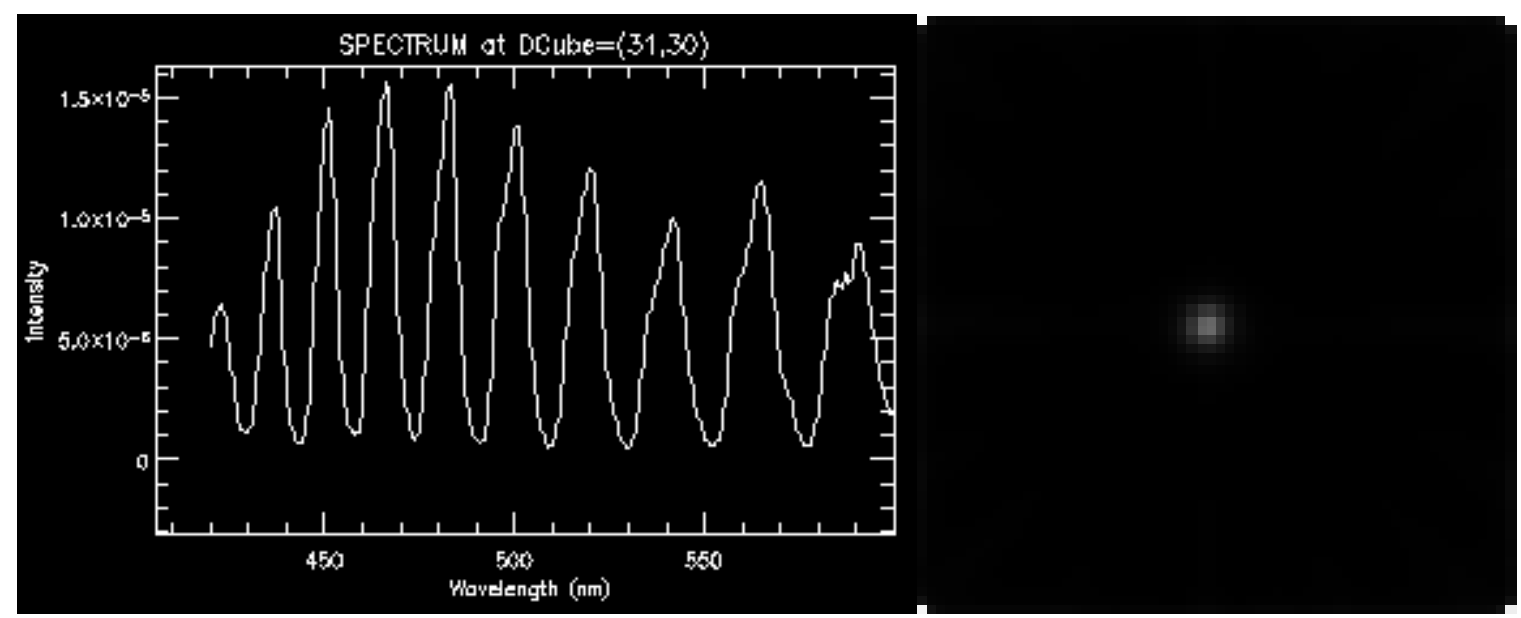

\section{Reconstruction Time $=\mathbf{4 3 6 . 9} \mathbf{~ s e c}$}

\section{ORIGINAL SYSTEM}

Figure 7.17: (left) The reconstructed spectrum of a white light point source polarized at 45 degrees in a visible CTICS system using existing $\mathrm{H}$ matrix with PSF calibration images. (right) Reconstructed zero order

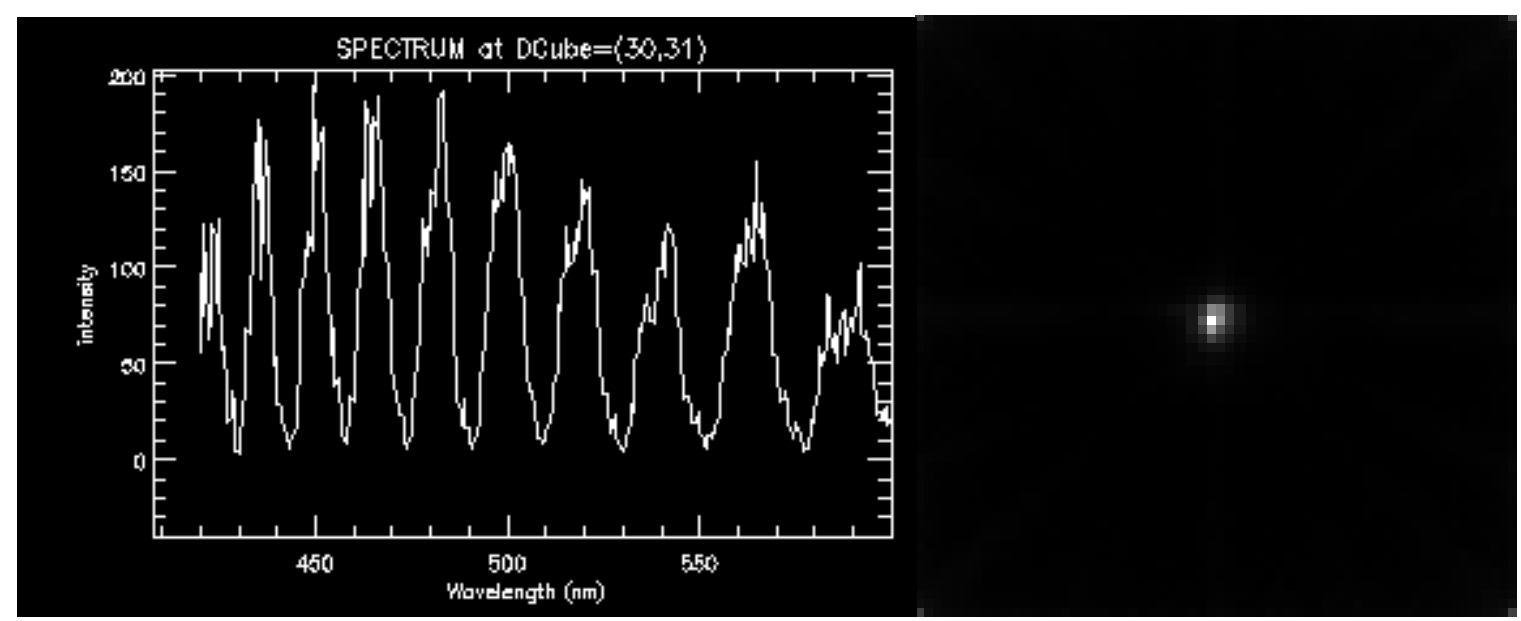

Reconstruction Time $=\mathbf{2 2 . 8}$ sec

MODIFIED H MATRIX

Figure 7.18: Reconstruction of same source using the newly modified dirac delta $\mathrm{H}$ matrix. Note that the reconstruction is nearly 20 times faster and still shows the same modulated spectral features. However, the spectral data from the original PSF H matrix is smoother.

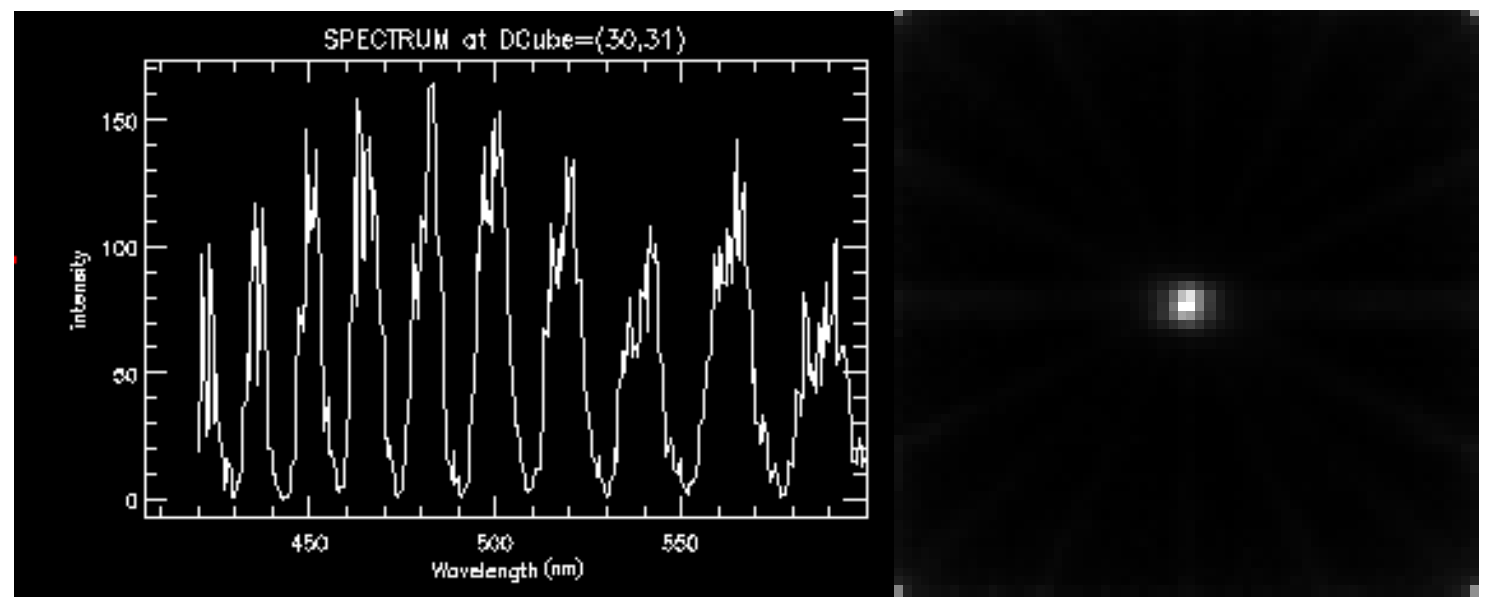

Reconstruction Time $=\mathbf{1 4 . 7} \mathbf{~ s e c}$

MODIFIED H MATRIX with only OUTER orders

Figure 7.19: Reconstruction of the same source using new H matrix, but only using the outer 16 diffraction orders. The resulting spectra is nearly identical to that in the center figure, but the reconstruction is now 1.5 times faster. 


\subsection{How to Implement the Dirac Delta Technique}

The goal of the dirac delta technique is to reduce the number of lit pixels in the CTIS system's H matrix. The following steps outline how the dirac delta technique can be implemented for a CTIS system in an attempt to meet this goal.

- Record CTIS calibration images following the standard process

- Calculate and record the PSF centroid location for each of the diffraction orders in each calibration image

- Fit a line to the centroid location in each diffraction order as a function of wavelength

- If possible, adjust the rotation of the $\mathrm{CGH}$ to improve the pixel alignment of the diffraction orders. In other words, try to get the line fit data for the horizontal and vertical orders to lay right along the exact pixel center locations across the entire diffraction order. If needed try to shift the object position when recording calibration images or apply a slight position shift to all of the diffraction order line fits (the same shift applied to all orders) to try to improve pixel registration.

- Use the line fit data to determine the calibration wavelengths that correspond to having centroids at the pixel center locations.

- Interpolate a signal consisting of a single lit pixel at the centroid line fit locations for the specified calibration wavelengths. (For wavelengths whose centroid corresponds to a focal plane location at the exact center of a pixel, the interpolation will result in a single lit pixel for that diffraction order in the synthetic calibration image. If the centroid is not at the exact pixel center, multiple pixels will be lit after this interpolation process.)

- Do this for all the diffraction orders and for all the calibration wavelengths, saving the synthetic calibration image for each wavelength. Use those synthetic calibration images to form the CTIS system dirac delta H matrix.

During this process it is important to utilize whatever methods are available to improve the CGH alignment in the system as well as improve the accuracy of the centroid calculations in order to ensure a proper line fit. Fine adjustment rotation stages can be used to help the CGH alignment, and other mathematical techniques and calibration methods may improve the accuracy of the centroid calculations. For example using an object with some spatial extent rather than a point object and/or the use of more advanced centroid estimation algorithms may provide further improvements.

In most cases it will be impossible to get perfect pixel alignment of the CGH diffraction orders to the FPA pixel array. When there is not a perfect pixel registration for the wavelength position in a given calibration image, multiple pixels will be lit in that diffraction order as opposed to one. Once this is done, the user may want to adjust or scale the relative intensities or pixel values in each synthetic calibration image based on some recorded system parameters to help improve smooth spectral reconstruction values. So when implementing the dirac delta technique, the user must make some selections based on the measured data to try to minimize the number of lit pixels in the synthetic calibration images and apply appropriate relative pixel values for each synthetic calibration image. 


\subsection{Using Deconvolution to Mitigate Spatial Resolution Tradeoff}

As a final note on the dirac delta reconstruction technique, the method does make a tradeoff of spatial resolution for an increase in reconstruction speed, but if higher spatial resolution is required additional deconvolution post processing steps can be applied to try to regain some of the lost spatial resolution at a cost of processing speed. There are a few different approaches on how to apply the deconvolution. A user could deconvolve either the known 2D point spread function for each wavelength in each monochromatic image slice of the reconstructed data or the user could deconvolve the $3 \mathrm{D}(\mathrm{x}, \mathrm{y}$, and $\lambda$ ) voxel spread function of the CTIS system. More details on the CTIS voxel spread function are described in a publication by Nathan Hagen. ${ }^{25}$

\subsection{Multiple Paths to Real Time CTIS Reconstruction}

The results outlined in this chapter provide a thorough description of current CTIS reconstructions and outline a clear step towards real time CTIS reconstruction. In fact, there are now multiple paths to achieve real time CTIS reconstructions through either the use of parallel processing or the use of some of the newly developed methods for sequential processing.

The parallel techniques outlined in the chapter can take advantage of the sparse nature of the CTIS H matrix, while separating out the necessary calculations into parallel processes through either the parallel calculation of the multiplication operations and additions operations or through combined fused multiply and add operations. Advancements in GPU architectures with hundreds of separate parallel processing cores should allow the necessary computer hardware for real time CTIS processing to become obtainable using these techniques with the possibility of having thousands of reconstructions to be calculated each second.

For sequential operations two methods have been proposed to create a CTIS that can perform reconstructions in real time using current computer hardware. The first method involves calculating a direct inverse of the CTIS H matrix, which has been shown feasible for small resolution CTIS systems that meet certain parameter requirements. A simulation of such a simple system using data from a constructed visible CTIS system along with a normal desktop computer, was able to demonstrate reconstructions that can be completed in $1 / 20^{\text {th }}$ of a second. If a CTIS were to be built matching these low resolution requirements, $6 \times 6$ pixels spatial resolution and 40 spectral bands, or if an existing CTIS were to be modified to meet these parameter requirements as outlined earlier in the chapter, demonstrations of a CTIS operating in real time at near video frame rates could be achievable today with standard computer hardware.

The second method, referred to as the dirac delta technique, has been tested and demonstrated on actual CTIS hardware and is able to increase the speed of reconstruction by over an order of magnitude by trading off some spatial resolution of the reconstructed data and modifying the calibration technique. The primary advantage to this method, as opposed to the first direct inverse method proposed, is that you are no longer constrained to simple CTIS systems. CTIS systems with hundreds of spectral bands and tens of hundreds of spatial resolution elements would see this same dramatic increase in reconstruction speed. CTIS reconstructions that had taken tens of minutes to complete can now be calculated in seconds or fractions of a second with this method bringing higher resolution CTIS much closer to real time operation. 


\section{CHAPTER 8: DETAILS AND RESULTS FROM THE LWIR CTIS}

\subsection{Demonstration of the LWIR CTIS System}

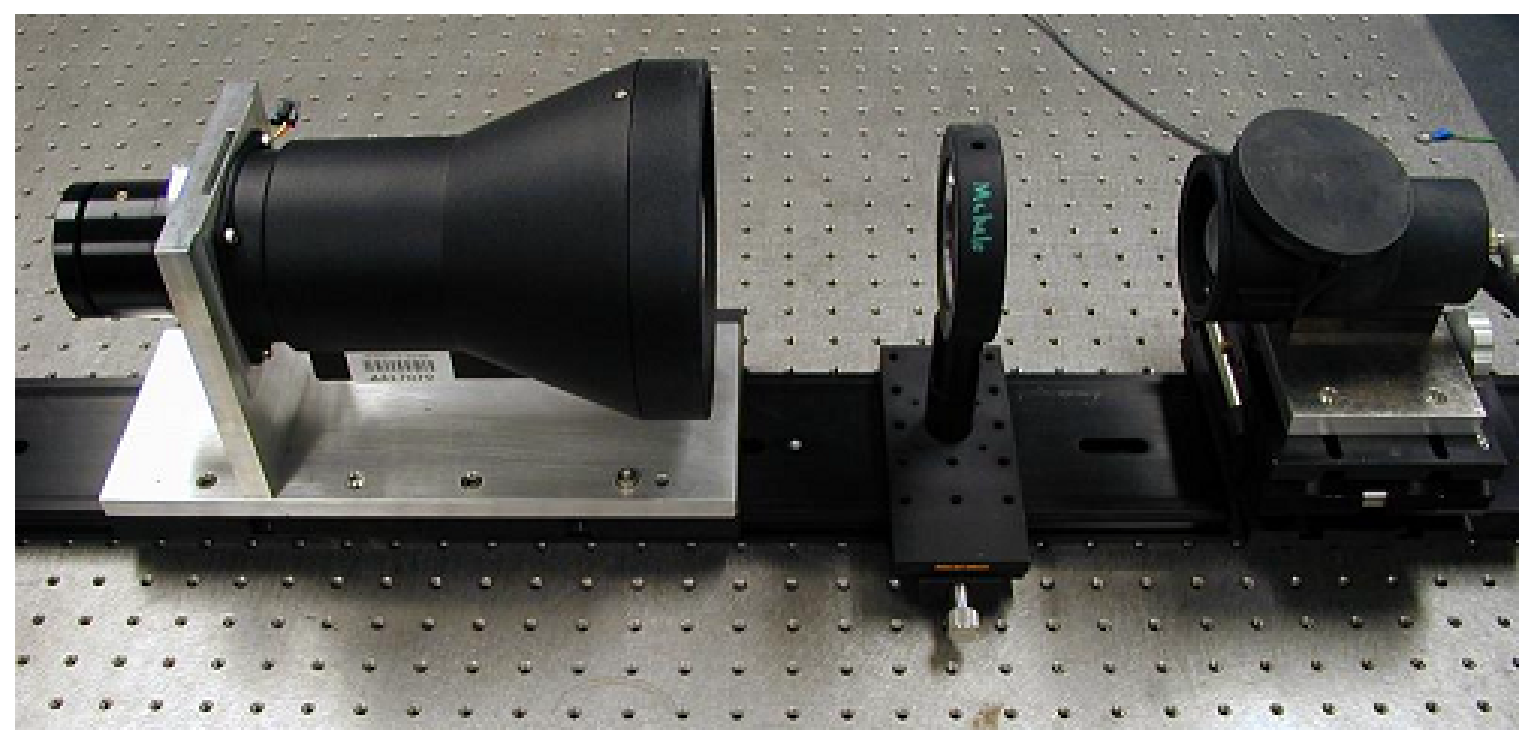

Figure 8.1: Photo of the assembled LWIR CTIS system

One of the main objectives of this dissertation work was to design and construct a proof-ofconcept snapshot imaging spectropolarimeter that works in the 8 to 12 micron LWIR region using a combination of Computed Tomographic Imaging Spectrometry (CTIS) and channeled spectropolarimetry. The construction of the system was separated into two parts. First, the LWIR CTIS snapshot spectrometer was assembled, tested, and demonstrated. Secondly, the three necessary polarization elements were manufactured and added to the LWIR CTIS system to incorporate channeled spectropolarimetry and complete the snapshot LWIR spectropolarimeter. This chapter will present some results from the constructed LWIR CTIS spectrometer system, shown in figure 8.1, while the following chapter will provide the theory of operation, design details, and demonstration results of the complete LWIR spectropolarimeter system.

The setup of the LWIR CTIS consists of three lenses, a field stop, a focal plane array, and a specialized Computer Generated Hologram (CGH) dispersing element. The objective and collimating lenses are commercial lenses from Axsys IR systems, with focal lengths of $50 \mathrm{~mm}$ and $150 \mathrm{~mm}$ respectively. The two lenses are placed in a custom made mount that also houses the small $2.0 \mathrm{~mm} \times 2.0 \mathrm{~mm}$ square aperture field stop at the intermediate image plane. The CGH disperser has been etched onto a 3-inch wafer of GaAs with a grating period of 288 microns and placed into a mount in the collimated beam space. Finally the reimaging lens and focal plane array are found in the high resolution $640 \times 480$ uncooled silicon microbolometer LWIR camera system from DRS-Nytech with a pixel pitch of 25 microns and integrated $62 \mathrm{~mm}$ focal length imaging lens. The specifications of all of the optical components were chosen to work in conjunction with the other elements to ensure that the CTIS pattern of 25 diffraction orders fits onto the focal plane array with minimal overlap. 
The results in the following figures show that the LWIR CTIS works, demonstrating its ability to simultaneously measure and determine the spectral signatures of all the spatial locations/targets in a scene with just a single frame of data. In figure 8.2, the LWIR CTIS is imaging the output of the exit slit of a monochromator. The reconstructed spectrum from a pixel location along the slit is shown on the right in figure 8.2 , demonstrating the monochromatic output and allowing the identification of the monochromator's wavelength output. For figure 8.3, the LWIR CTIS is viewing the output from a blackbody source that has a unique spectral feature. It would be extremely difficult, if not impossible, for a standard panchromatic LWIR camera to identify this target based on this spectral feature, however, the LWIR CTIS clearly demonstrates that a 9.4 micron wavelength cut-on spectral filter had been placed in front of the blackbody source.
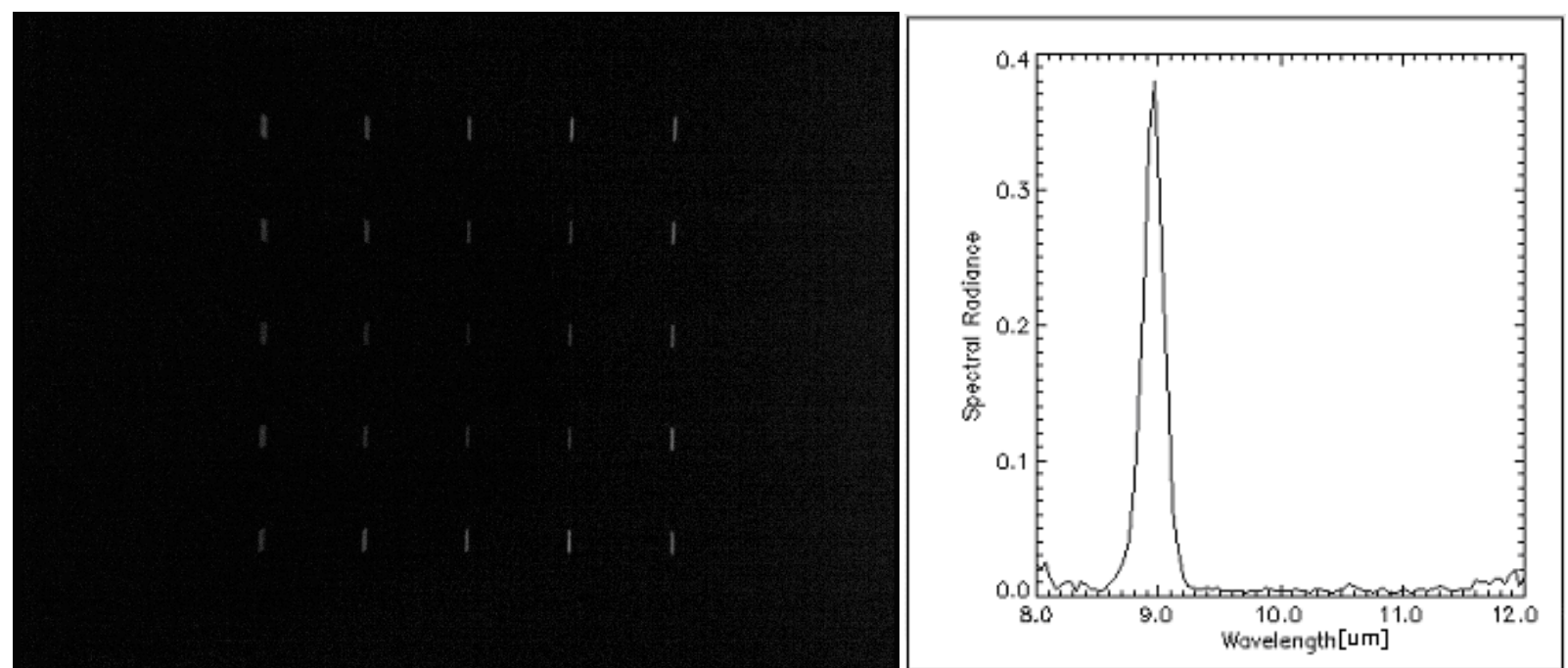

Figure 8.2: (left) The raw image captured by the LWIR CTIS when viewing the exit slit of a monochromator set to have a 9 micron wavelength output. (right) The spectral reconstruction of the exit slit.
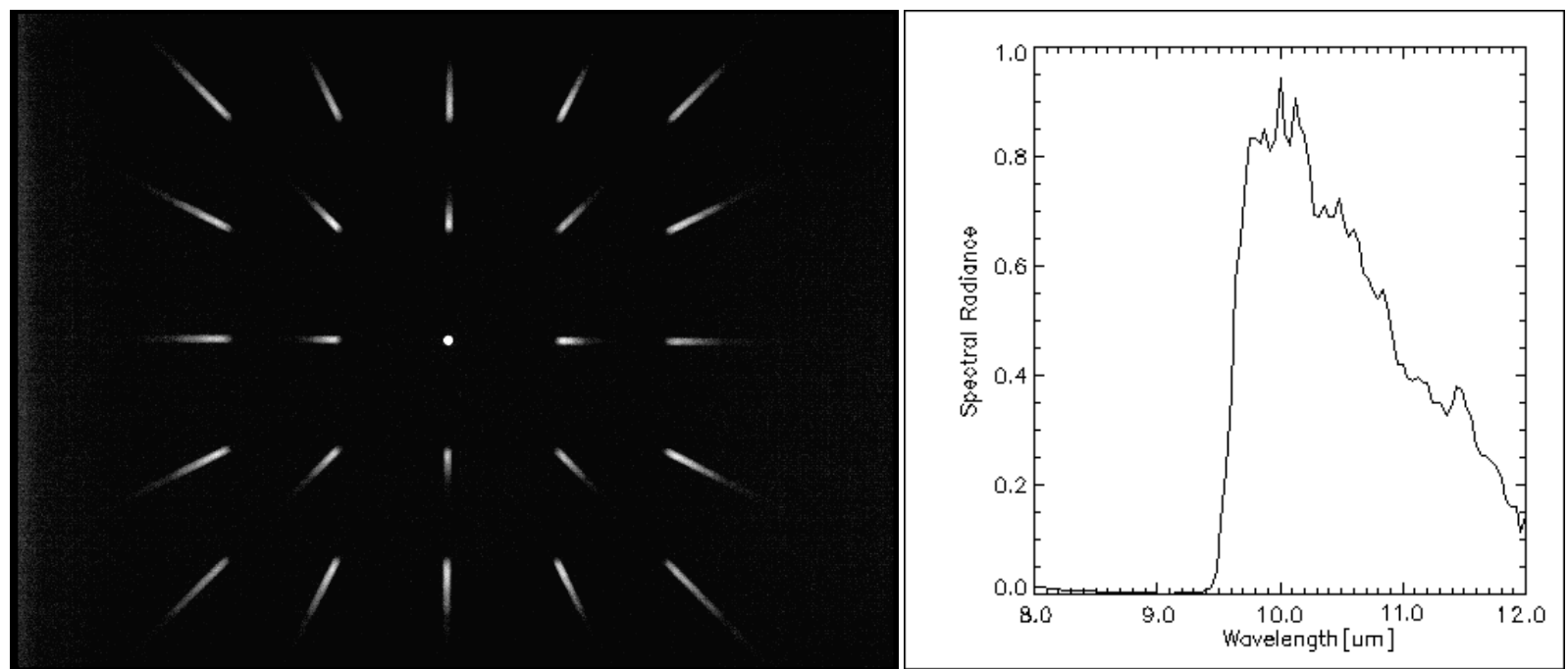

Figure 8.3: (left) The raw image captured by the LWIR CTIS when viewing a 1000 degree C blackbody through a 9.4 micron cut on spectral filter. (right) The spectral reconstruction of the exit port of the filtered blackbody. 
While the previous examples consisted of static scenes with only a single target, the results shown in figures 8.4 and 8.5 demonstrate the LWIR CTIS system's ability to record complete spatial and spectral data for dynamic events that change quickly and for scenes with multiple targets. In figure 8.4 the LWIR CTIS is viewing two targets, the tip of a hot soldering iron and the output spot from a $\mathrm{CO} 2$ laser. Even though the scene was spatially static, its spectral properties were changing in time, since the wavelength output of the $\mathrm{CO} 2$ laser was unstable and would shift in time. For a brief moment in time, lasting only a fraction a second, the unstable laser output was actually lasing in two spectral modes. The LWIR CTIS was able to record and measure the spatial and spectral properties of both targets during this event in the single frame of data shown below.

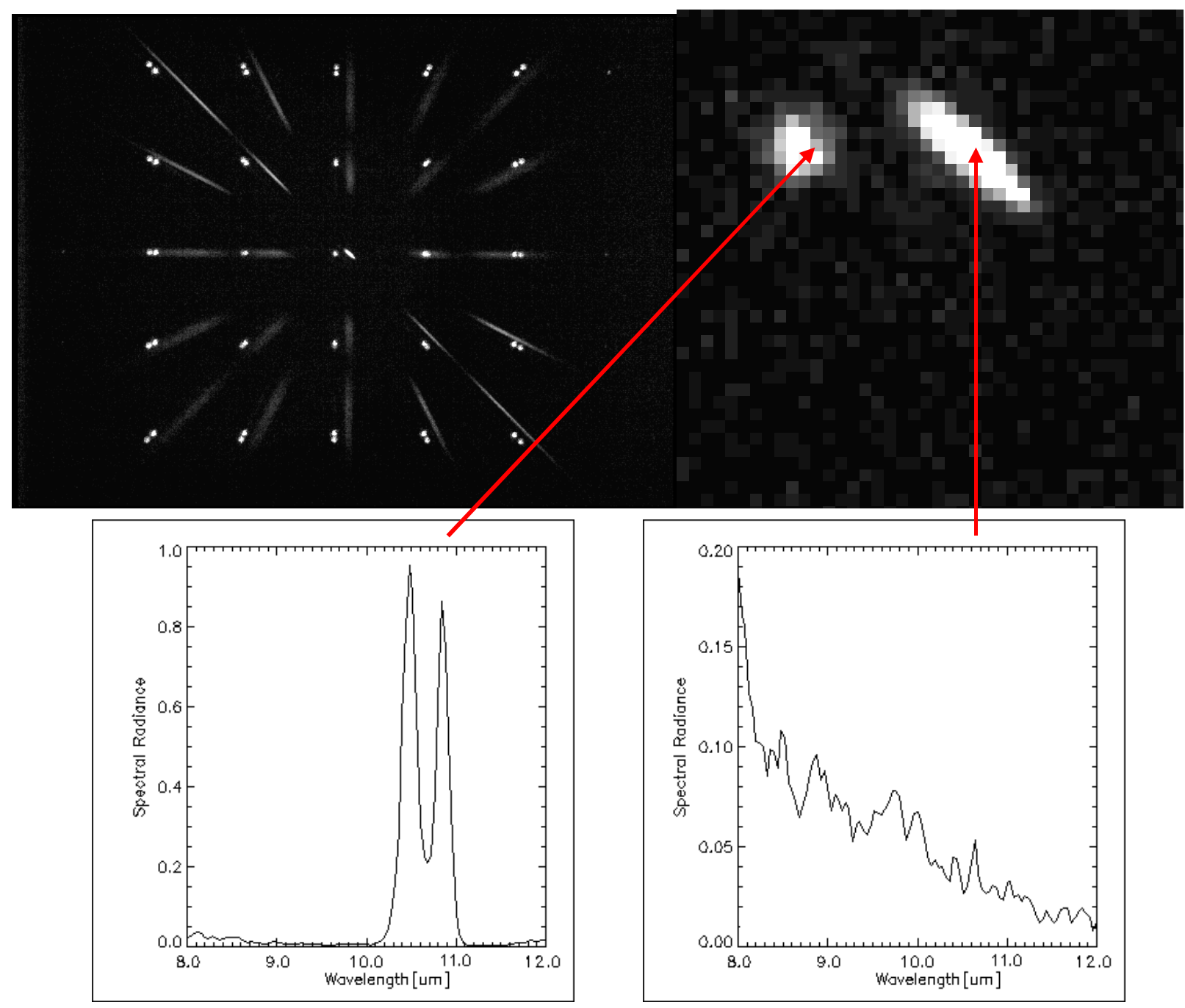

Figure 8.4: (top left) The raw CTIS image viewing the tip of a soldering iron and output of a $\mathrm{CO} 2$ laser. (top right) Zero order image. (bottom left) The spectral reconstruction of light from the $\mathrm{CO} 2$ laser. The light output from the laser wasn't stable, and the lasing wavelength of the laser would vary in time. In this instance you can see that the laser was actually lasing in two spectral modes, which only occurred for a very short period of time, providing an excellent demonstration of the snapshot capability of the CTIS system. (bottom right) The blackbody like spectral output from the tip of the soldering iron. 
In figure 8.5 the LWIR CTIS was viewing a scene consisting of three objects at different spatial locations. The three objects were the laser light output from a CO2 laser, the tip of a hot soldering iron, and a separate portion of a hot soldering iron that was covered by a spectral filter. This test setup was intended to demonstrate the LWIR CTIS systems ability to provide simultaneous multiple target characterization, classification, and identification based on the recorded spatial and spectral properties of the scene. The reconstructed spectral data from each spatial location in the recorded zero order is clearly able to identify these three objects based on their spectral properties as shown in the results below.

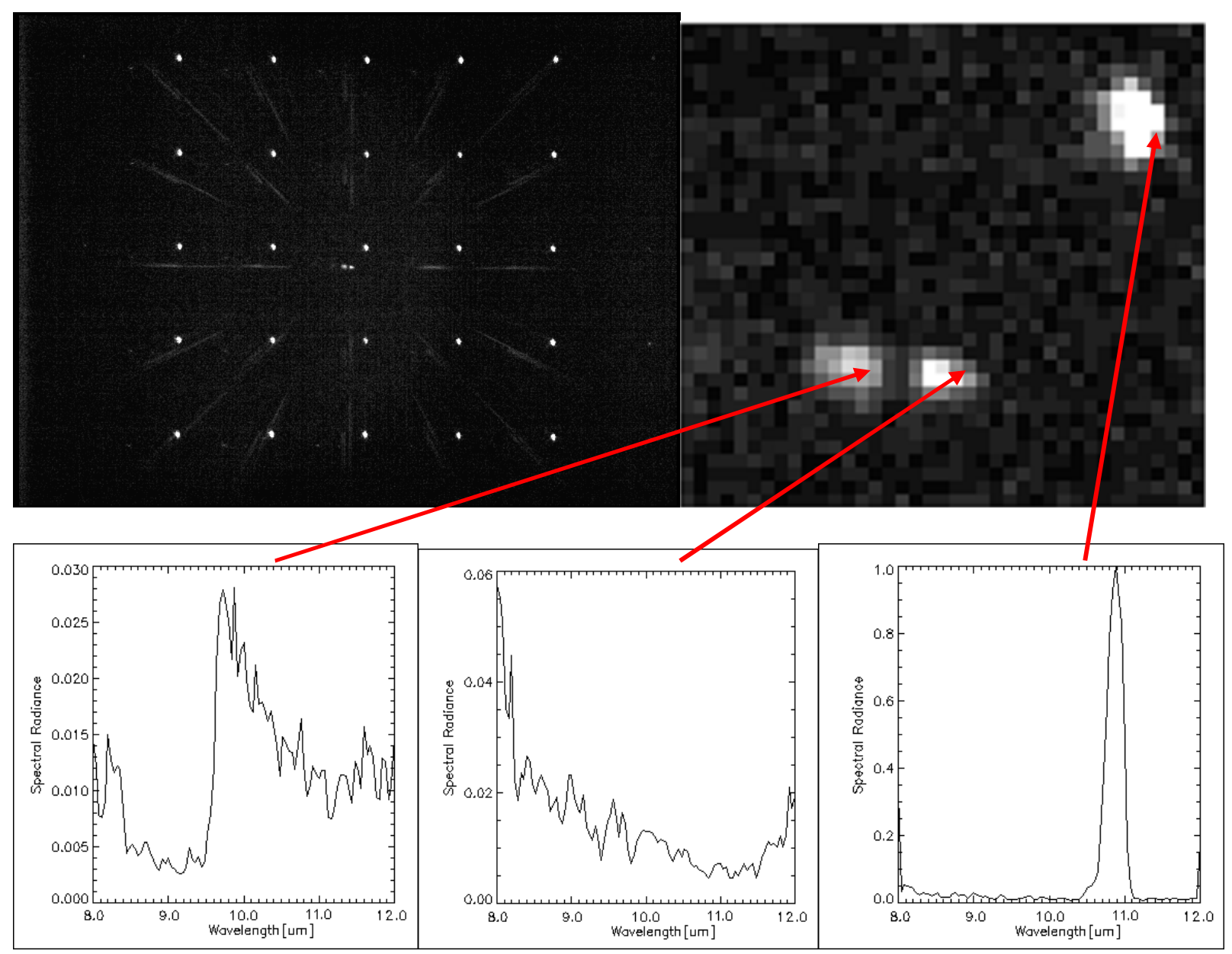

Figure 8.5: (top left) The raw CTIS image viewing CO2 laser light and the tip of a soldering iron where part of the soldering iron is covered by a 9.4 micron cut on spectral filter. (top right) Zero order image. (bottom left) The spectral reconstruction of this point clearly shows that this was the part of the soldering iron covered by the spectral filter. You can clearly see the spectral features due to the 9.4 micron cut on filter. (bottom middle) The blackbody like spectral output, indicating that this target was the unfiltered tip of the soldering iron. Note that the data points at the extremes of the spectrum suffer from artifacts of the iterative tomographic reconstruction process. (bottom right) The spectral reconstruction of the monochromatic $\mathrm{CO} 2$ laser light. 


\section{CHAPTER 9: CTICS DETAILS AND DESIGN}

\subsection{Channeled Spectropolarimetry \& CTIS}

The results from the previous chapter demonstrate that the constructed LWIR CTIS works. The optical system is able to function as a snapshot imaging spectrometer, providing the spectra from 8-12 microns for all of the spatial locations of the scene with just a single frame of data. The capabilities of this proof of concept system can be extended further to include polarization measurements by combining channeled spectropolarimetry with the LWIR CTIS. The resulting system will be an LWIR snapshot imaging spectropolarimeter, able to simultaneously record both the spectral and polarimetric signatures of all the spatial locations/targets in a scene with one integration period of the camera.

\subsection{Incorporating Channeled Spectropolarimetry}

To incorporate channeled spectropolarimetry into a CTIS system only three polarization elements need to be added. The optical layout for the system is shown in figure 9.1. The added elements are placed in the collimated space of the CTIS, before the CGH, and consist of two thick birefringent crystal retarders, with their fast axes oriented at 0 and 45 degrees respectively, and an analyzing polarizer oriented at 0 degrees. These added components will encode the polarization information of the light into intensity modulations across the spectra as shown in figure 9.2. By carefully analyzing these recorded intensity modulations, the polarization state of each wavelength band in the spectrum can be determined at all the spatial locations of the scene. We refer to this combined system as a Computed Tomographic Imaging Channeled Spectropolarimeter (CTICS).

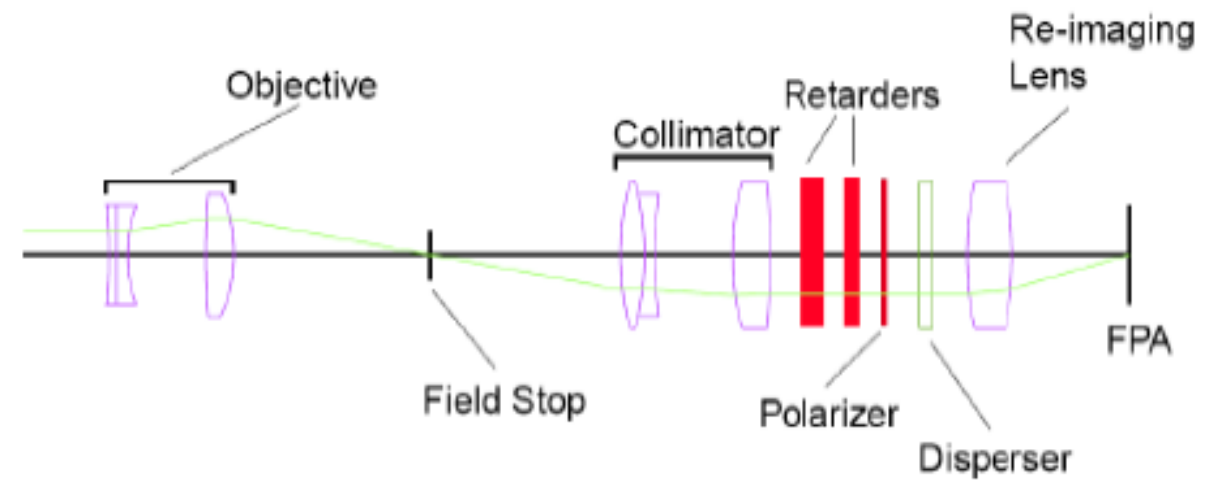

Figure 9.1: Optical layout for CTICS 


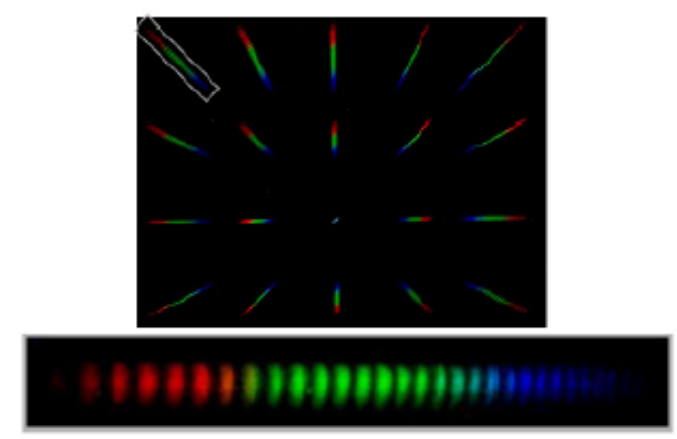

Figure 9.2: Example of modulated spectra recorded when viewing a polarized broadband object with a visible CTICS system.

We can see how the spectral dependence of the Stokes components are encoded into intensity modulations in the spectra by analyzing the added components of the system. Mueller matrices and stokes vectors provide an ideal way to analyze the electric field output of the light leaving the set of polarization elements. The following equation uses the Mueller matrices for the added optical elements to determine the Stokes vector output from the elements with an arbitrary polarization input.

$$
S_{\text {out }}=\left(\begin{array}{cccc}
1 / 2 & 1 / 2 & 0 & 0 \\
1 / 2 & 1 / 2 & 0 & 0 \\
0 & 0 & 0 & 0 \\
0 & 0 & 0 & 0
\end{array}\right)\left(\begin{array}{cccc}
1 & 0 & 0 & 0 \\
0 & \cos \left(\delta_{2}\right) & 0 & -\sin \left(\delta_{2}\right) \\
0 & 0 & 1 & 0 \\
0 & \sin \left(\delta_{2}\right) & 0 & \cos \left(\delta_{2}\right)
\end{array}\right)\left(\begin{array}{cccc}
1 & 0 & 0 & 0 \\
0 & 1 & 0 & 0 \\
0 & 0 & \cos \left(\delta_{1}\right) & \sin \left(\delta_{1}\right) \\
0 & 0 & -\sin \left(\delta_{1}\right) & \cos \left(\delta_{1}\right)
\end{array}\right)\left(\begin{array}{c}
s_{0} \\
s_{1} \\
s_{2} \\
s_{3}
\end{array}\right)
$$

Note that $\delta_{1}$ and $\delta_{2}$ correspond to the retardance of the first and second optical retarders, and that both of these quantities vary as a function of wavelength. Specifically the retardance is given by:

$$
\delta=2 \pi * \mathrm{OPD}^{*} \sigma=2 \pi * \mathrm{~d}^{*} \Delta \mathrm{n}^{*}(1 / \lambda)
$$

Where $\mathrm{d}$ is the thickness of the material and $\Delta \mathrm{n}$ is the difference in index of refraction between the ordinary and extraordinary axes of the material at the given wavelength $\lambda$. The quantity $1 / \lambda$ is equal to the wavenumber of light and is often expressed with the symbol $\sigma$.

Multiplying the matrices reveals that the output stokes vector is given by:

$$
s_{\text {out }}=1 / 2\left(\begin{array}{c}
s_{0}+s_{1} \cos \left(\delta_{2}\right)+s_{2} \sin \left(\delta_{1}\right) \sin \left(\delta_{2}\right)-s_{3} \cos \left(\delta_{1}\right) \sin \left(\delta_{2}\right) \\
s_{0}+s_{1} \cos \left(\delta_{2}\right)+s_{2} \sin \left(\delta_{1}\right) \sin \left(\delta_{2}\right)-s_{3} \cos \left(\delta_{1}\right) \sin \left(\delta_{2}\right) \\
0 \\
0
\end{array}\right)
$$

Since the detector itself is only sensitive to intensity and not to a particular polarization state, it only responds to the first component of the stokes vector, which corresponds to the total intensity 
of the light leaving the polarizer. This recorded intensity is a function of wavelength/wavenumber and is given by the equation:

$$
2 I(\sigma)=s_{0}+s_{1} \cos \left(\delta_{2}\right)+s_{2} \sin \left(\delta_{1}\right) \sin \left(\delta_{2}\right)-s_{3} \cos \left(\delta_{1}\right) \sin \left(\delta_{2}\right)
$$

This equation describes how the addition of the two retarders and polarizer allow the four stokes components, which also vary as a function of wavenumber, to be encoded as intensity modulations in the recorded spectra. Consider the case where you are imaging a spectrally uniform, broadband point object that is horizontally polarized across the entire spectrum $\left(\mathrm{s}_{0}=1\right.$, $\left.\mathrm{s}_{1}=1, \mathrm{~s}_{2}=0, \mathrm{~s}_{3}=0\right)$. You can see from the equation that the intensity modulation will vary as the cosine of the retardance of the second retarder, which is linear with wavenumber. If the polarization properties of the object were different, the resulting modulations in the spectra would also be different, given by the previous equation. These modulations will appear in the diffraction orders of the CTICS system as shown in figure 9.3.
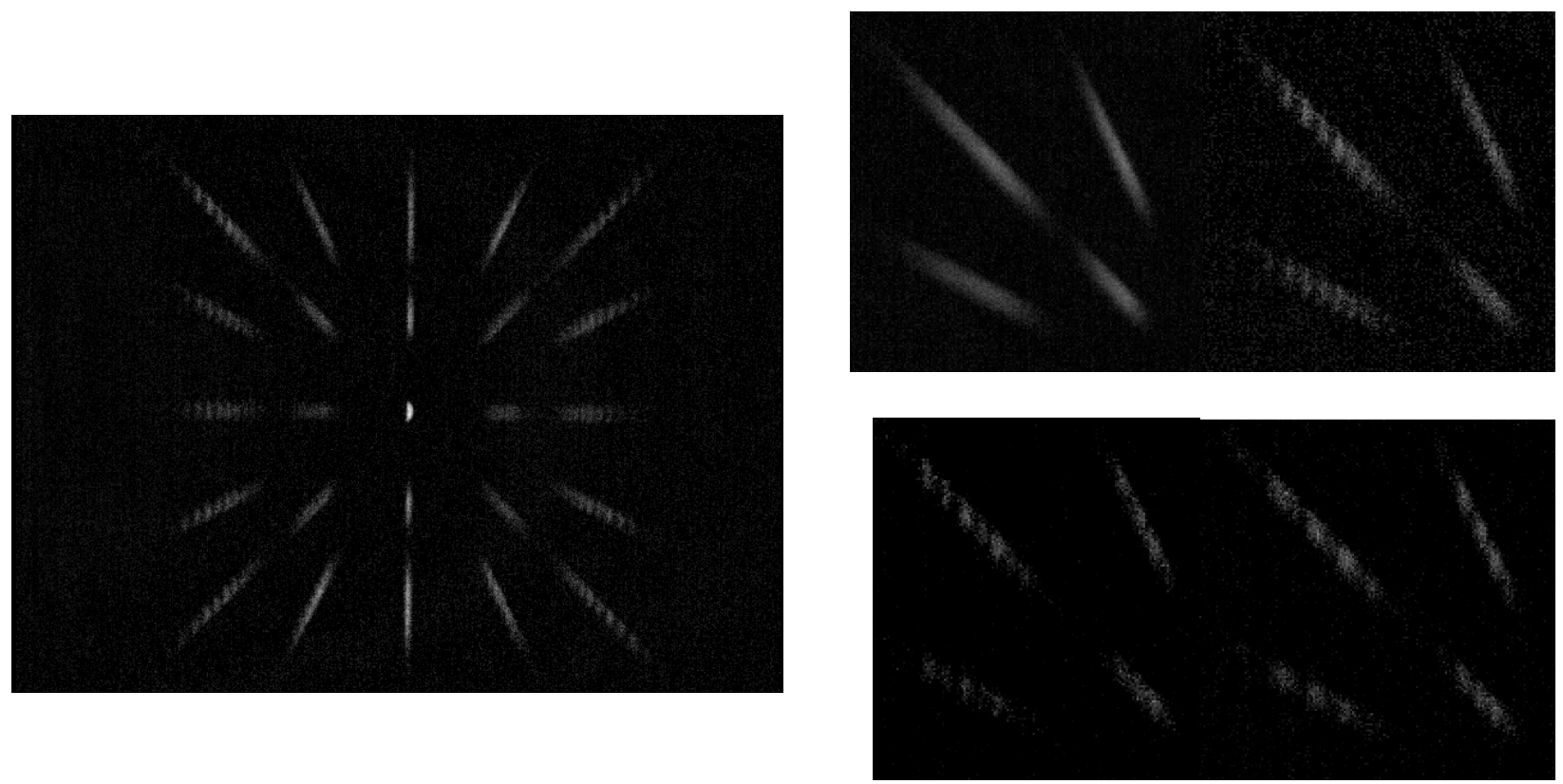

Figure 9.3: (left) Raw image acquired by LWIR CTICS when viewing a horizontally polarized blackbody object. Note the intensity modulations in the spectra for each of the diffraction orders (right) Zoomed in image of diffraction orders demonstrating how the modulation changes depending upon objects polarization state; unpolarized (top center), horizontally polarized (top right), vertically polarized (bottom center), and linearly polarized at 45 degrees (bottom right). 
Euler's identity:

$$
\begin{aligned}
& \cos (\alpha)=\frac{1}{2}\left(e^{i \alpha}+e^{-i \alpha}\right) \\
& \sin (\alpha)=\frac{-i}{2}\left(e^{i \alpha}-e^{-i \alpha}\right)
\end{aligned}
$$

In order to reconstruct the four stokes components spectra from a recorded modulated spectrum, we first use Euler's identity to rewrite the equation describing the spectral intensity modulation function. The result is given below.

$$
\begin{aligned}
& I(\sigma)=\frac{1}{2} s_{0}+\frac{1}{4} s_{1}\left(e^{i \delta_{2}}+e^{-i \delta_{2}}\right)+\frac{1}{8}\left[\left(s_{2}-i s_{3}\right) e^{i\left(\delta_{2}+\delta_{1}\right)}+\right. \\
& \left.\left(s_{2}+i s_{3}\right) e^{-i\left(\delta_{2}+\delta_{1}\right)}+\left(-s_{2}-i s_{3}\right) e^{i\left(\delta_{2}-\delta_{1}\right)}+\left(-s_{2}+i s_{3}\right) e^{-i\left(\delta_{2}-\delta_{1}\right)}\right]
\end{aligned}
$$

In this re-expressed form of the equation we see that the intensity is composed of seven different frequency components based on the retardance of the two added optical retarders: $0, \pm \delta_{2}, \pm\left(\delta_{2}\right.$ $\left.+\delta_{1}\right)$, and $\pm\left(\delta_{2}-\delta_{1}\right)$. If the same material is used for both retarders, and the second retarder is twice as thick as the first, then these seven frequency channels will be evenly spaced apart since $\delta_{2}$ will equal $2 \delta_{1}$. By taking the Fourier transform of the measured intensity pattern, we can easily see the seven separated frequency channels as shown in figure 9.4.

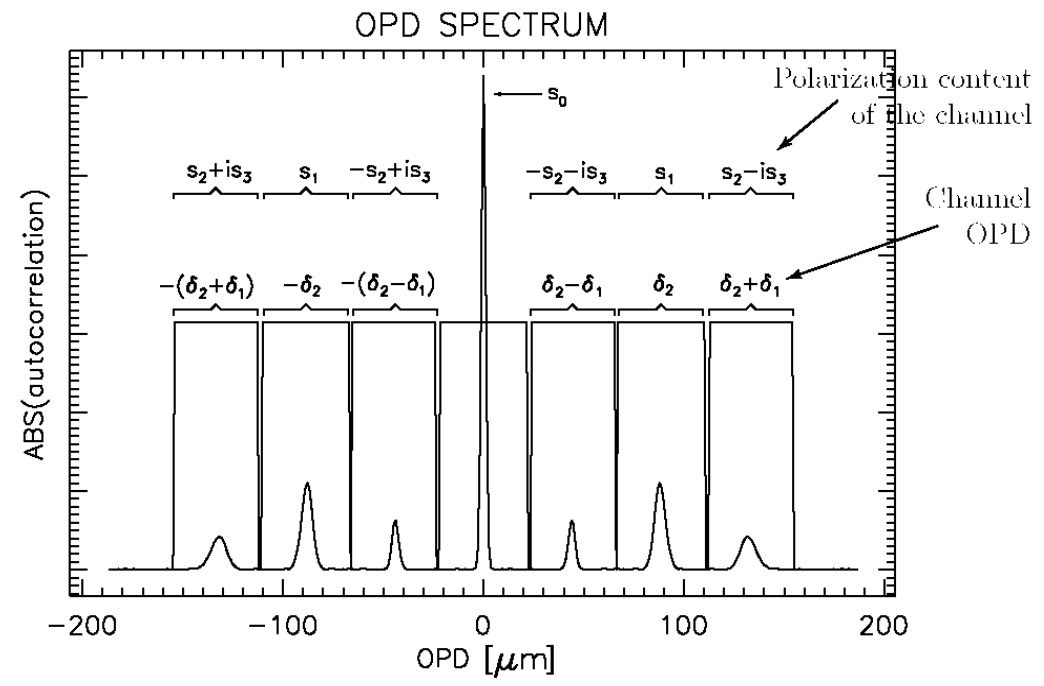

Figure 9.4: Plot showing the 7 frequency channels that compose the wavelength intensity function.

Note that in the same way the Fourier transform is often used to transform a function of time (units of seconds) into a new function whose argument is frequency (units of inverse seconds), this use of the transform is transforming a function of wavenumber (units of inverse meters) into a new function whose argument is OPD (units of meters) due to the retarders. So the "frequency channels" illustrated in figure 9.4, and reprinted in figure 9.5, are bands of OPD, much in the same way that OPD can be thought of as the analogous "frequency space" to the initial intensity 
spectrum which was a function of wavenumber. These channels are separated since the transform of a function times a complex exponential can be found using the frequency shift property.

Each of these seven frequency channels corresponds to the intensity spectrum of a particular polarization state or a combination of polarization states. For example, signal within the zero frequency channel is only composed of signal from unpolarized light, $\mathrm{s}_{0}$, while the $\pm \delta_{2}$ frequency channels are composed of light that is only $\mathrm{s}_{1}$ polarized. To reconstruct the polarization data across the spectrum for a given stokes component, we must first select the desired frequency channel, multiply by an appropriate windowing function to isolate the channel, and then inverse Fourier transform to see how that polarization state varies in wavenumber. To reconstruct the $s_{2}$ and $s_{3}$ stokes components you must add or subtract data from the appropriate frequency channels before applying an inverse Fourier transform.

It is the fact that the different stokes components encode modulations of the spectra at different frequencies, that allows us to reconstruct the spectral dependence of the four stokes components across spectrum.

\subsection{Channeled Spectropolarimetry in CTICS}

When channeled spectropolarimetry in incorporated into a CTIS system, the imaging system will now be able to determine the four stokes components for all the wavelength bands in addition to determining the spectra from each spatial location in a scene.

To be clear, this analysis is done in two separate steps. First, the same tomographic reconstruction techniques, as in a regular CTIS, are used to reconstruct the spectra for each spatial position. Due to the added polarization elements in the system, these reconstructed spectra will contain intensity modulations depending upon the polarization properties of the incident light. In the next step, these modulated spectra are then individually analyzed using the Fourier technique outlined above to reconstruct the four stokes components spectra for each spatial location.

In order to obtain accurate reconstructions of the polarization state as a function of wavelength, we will require an accurate measurement of the spectral intensity modulations as well as an accurate description of the properties of the retarders.

\subsection{Selection of Retarders for CTICS}

The primary challenge faced when designing a CTICS instrument is the selection of the two retarders and their corresponding thicknesses. This selection will depend upon properties of the system, properties of the retarder, and the types of objects viewed with the system. There is no single "right answer" for the selected retarders and their thicknesses. Often times a range of values can be used. There will, however, be a maximum set of retarder thicknesses corresponding to the point where the produced modulations can no longer be accurately recorded across the entire spectra that can be used as a starting point in selecting retarder thicknesses. 
To begin the investigation of the selection of these parameters, lets first refer to figure 9.5 and consider how the retarders affect the frequency channels of the modulated spectrum. The figure illustrates that if the second retarder is twice as thick as the first, then the 7 frequency channels are equally spaced apart. The figure also shows that all four of the stokes spectra can be reconstructed with just the information contained in the inner 5 frequency channels. The higher frequency information contained in the outermost channels is not needed for reconstruction.

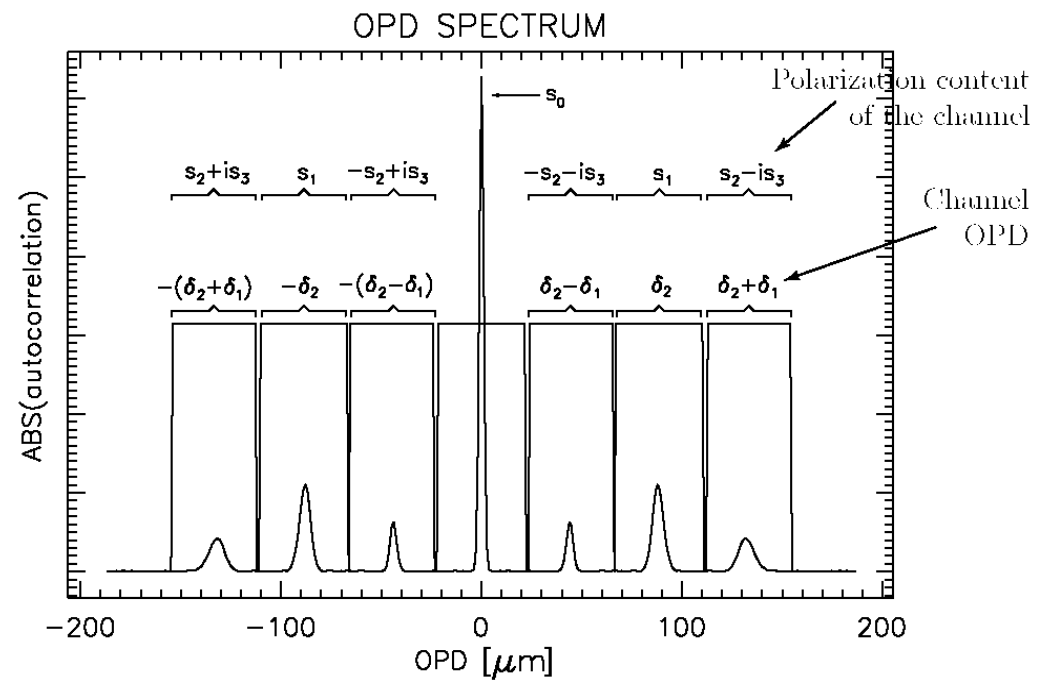

Figure 9.5: Plot showing the 7 frequency channels that compose the wavelength intensity function.

Also recall that the plot shown in figure 9.5 is the result of the Fourier transform of the recorded intensity as a function of wavenumber, which is given by equation (9.4) shown below:

$$
2 I(\sigma)=s_{0}+s_{1} \cos \left(\delta_{2}\right)+s_{2} \sin \left(\delta_{1}\right) \sin \left(\delta_{2}\right)-s_{3} \cos \left(\delta_{1}\right) \sin \left(\delta_{2}\right)
$$

where $\delta_{1}$ and $\delta_{2}$ are the retardance of the first and second retarders, which are related to the thickness of the retarders, $d$, as shown in the following equations.

$$
\begin{aligned}
& \delta_{1}=2 \pi d_{1} \Delta n \sigma \\
& \delta_{2}=2 \pi d_{2} \Delta n \sigma
\end{aligned}
$$

In the same way that the function $\cos (3 \mathrm{x})$ has a higher frequency and a smaller period of oscillation than the function $\cos (\mathrm{x})$, the thickness of a retarder is related to how quickly the intensity is modulated across the spectrum. By looking at the equations above, you can see that increasing the thickness of a retarder, $\mathrm{d}$, increases the retardance, $\delta$, which results in a higher frequency of oscillation or an increase in the number of intensity modulations across the spectrum. So the period of the oscillations will be smaller for thicker retarders since the intensity has a $\cos (\delta)$ and $\sin (\delta)$ dependence and the retardance is linearly proportional to wavenumber. This also implies the spacing of the frequency channels will be further apart for thicker retarders and closer together for a set of thinner retarders. 
There will be a limit to the number of oscillations the CTICS system can detect, and therefore there will be a corresponding limit to how thick the retarders can be. This is important because the quality of the polarization reconstructions of the CTICS depend upon an accurate measurement of the spectral intensity modulations, at least in the center 5 frequency channels. For example, since the pixels on the detector have a finite size, intensity modulations with a period smaller than the length of two pixels cannot be accurately recorded. The higher frequency information will be aliased to lower frequencies. The spectral resolution of the spectrometer system will have a similar effect on the modulation/retarder thickness limit. Since the modulated spectra for each spatial position must first be reconstructed from the image before the polarization components can be determined, any modulations produced by the retarders with a period smaller than the spectral resolution of the system will not be accurately shown in the reconstructed data. This is significant because the spectral resolution of the spectrometer system is object dependent. ${ }^{26}$ Point objects have a higher spectral resolution than objects with larger spatial extents, so the selection of retarders should take into account the types of objects the system will view. Noise in the recorded CTICS image will also affect the quality of the stokes reconstructions.

Because of all these reasons, there is no one specific "right answer" for the selection of the retarder parameters. The designer must consider factors such as the properties of the optical system and detector as well as the characteristics of objects viewed with the system when selecting the polarization components. Ultimately the selection of retarders and retarder thicknesses must be made such that when viewing a particular scene, the number of recorded modulations/fringes is maximized and that the produced modulations across the spectra are accurately measured by the system.

To accomplish this, the designer must select the position of the outermost frequency channel used in reconstruction such that there is enough signal and data in each of the frequency channels to meet the performance requirements for the system. Due to the MTF of the overall system, higher frequency data near this cutoff while have a much lower SNR than lower frequency data and may not be accurately measured due to noise. When selecting retarder thicknesses, it is useful to first find the modulation limit where the produced fringes are no longer accurately measured. This modulation limit can then be associated with a particular maximum frequency of the seven frequency channels and a corresponding set of maximum retarder thicknesses. These thickness values can then be scaled back to slightly thinner values to try and meet the system performance requirements.

When determining the specific cutoff point where modulations are no longer accurately measured, note that the intensity modulation is linear as a function of wavenumber not wavelength. For a constant frequency modulation in wavenumber, the intensity modulations as a function of wavelength are much tighter at smaller wavelengths become progressively more stretched out at longer wavelengths, since wavenumber is equal to one over wavelength. So the period of oscillation is smallest near the lowest wavelength or "blue" end of the recorded spectrum and gradually increases at higher wavelengths or the "red" end of the spectrum.

Since the focal plane of the CTICS samples evenly in wavelength, and provided the birefringence of the material is constant, this means that the first place where the spectra will no 
longer be accurately measured will occur at the lowest wavelength of the system when the period of oscillation is greater than two times the resolution/sampling rate of the system.

As an example, consider the following steps used to calculate a set of retarder thicknesses for an LWIR CTICS system, operating from 8-12 micron, and viewing broadband point objects.

$$
\lambda_{\min }=8000 \mathrm{~nm}
$$

We want the period of the highest frequency component of the spectral modulations to be equal to the spectral resolution of the system at $\lambda_{\min }$. For a point object, the spectral resolution is given by 2 times the spectral sampling rate at the lowest wavelength. The LWIR CTICS has a minimum spectral sampling rate of $60 \mathrm{~nm}$ in the outermost diffraction orders, so the spectral resolution is $120 \mathrm{~nm}$. If the system were to view extended area objects this spectral resolution would need to be increased.

\section{$\Delta \lambda=120 \mathrm{~nm}$}

Next, we need to find the corresponding change in wavenumber for this change in wavelength at the minimum wavelength. The change in wavenumber can be determined by substituting in $\lambda_{2}=$ $\lambda_{1}+\Delta \lambda$ into the rewritten change in wavenumber expression as follows:

$$
\begin{aligned}
& \Delta \sigma=\sigma_{1}-\sigma_{2}=\left(\frac{1}{\lambda_{1}}\right)-\left(\frac{1}{\lambda_{2}}\right)=\frac{\lambda_{2}-\lambda_{1}}{\lambda_{1} \lambda_{2}} \\
& \lambda_{2}=\lambda_{1}+\Delta \lambda \\
& \Delta \sigma=\frac{\Delta \lambda}{\lambda^{2}+\lambda \cdot \Delta \lambda}
\end{aligned}
$$

So the minimum $120 \mathrm{~nm}$ change in wavelength at the lowest wavelength of 8 microns corresponds to a change in wavenumber of:

$$
\Delta \sigma=\frac{(120 \mathrm{~nm})}{(8000 \mathrm{~nm})^{2}+(8000 \mathrm{~nm}) \cdot(120 \mathrm{~nm})}=1.847 \times 10^{-6} \mathrm{~nm}^{-1}
$$

We now want the highest frequency component of the spectral modulation in wavenumber space to have a period of $\Delta \sigma$.

To maximize the amount of data in each of the frequency channels, we want to stretch the channels out as much as possible yet still be able to accurately record at least the inner 5 frequency channels. To do this we place the center of the outermost frequency channels at the modulation limit. The width of each channel is a fixed value, equal to the OPD of the first retarder $\delta_{1}$, so the center of the outermost channels corresponds to an OPD of \pm three times the OPD of the first retarder $\delta_{1}$. With the modulation limit at this center location, half of the outermost channel, composed of frequencies higher than the modulation limit, will be aliased to 
the lower half of that same channel. The inner 5 channels should be minimally affected and should still accurately describe the lower modulation frequencies.

The center of the outermost frequency channel corresponds to a cosine modulation of three times the retardance of the first, thinner retarder.

$$
\cos \left(3 \delta_{1}\right)=\cos \left(32 \pi \mathrm{OPD}_{\text {retarder } 1} \sigma\right)=\cos \left(2 \pi 3 \mathrm{OPD}_{\text {retarder } 1} \sigma\right)=\cos \left(2 \pi 3 d_{1} \Delta n \sigma\right)
$$

This modulation has a period of $1 /(3 \mathrm{~d} \Delta \mathrm{n})$

We want $\cos \left(3 \delta_{1}\right)$ to have a period of $\Delta \sigma$ in wavenumber space. By setting $\Delta \sigma$ equal to the period of $\cos \left(3 \delta_{1}\right)$ we can solve for the thickness of the first retarder.

$$
\Delta \sigma=\frac{1}{3 d_{1} \Delta n} \Rightarrow d_{1}=\frac{1}{3 \Delta \sigma \Delta n}
$$

By substituting in for $\Delta \sigma$, the thickness of the first retarder can be rewritten as:

$$
d_{1}=\frac{\lambda_{\min }^{2}+\lambda_{\min } \Delta \lambda}{3 \Delta \lambda \Delta n}
$$

For a material where the difference in index of refraction between the ordinary and extraordinary axes/birefringence has a constant value of 0.014 as a function of wavelength, the corresponding thickness of the first and second retarder can be calculated as follows.

$$
\begin{aligned}
& d_{1}=\frac{(8000 \mathrm{~nm})^{2}+(8000 \mathrm{~nm})(120 \mathrm{~nm})}{3(120 \mathrm{~nm})(0.014)}=12.89 \mathrm{~mm} \\
& d_{2}=2 d_{1}=25.78 \mathrm{~mm}
\end{aligned}
$$

If the designer did not want to stretch the channels out but instead wanted to try and record all of the modulations then the edge of the outermost frequency channel would be placed at the modulation limit instead of the center of the channel. The resulting calculation for the thickness of the retarders would be the same, but would have a factor of 3.5 in the denominator instead of a factor of 3 .

Increasing or decreasing the retarders to stretch or contract the frequency channels is related to the stokes spectra resolution of the system because the information of how a particular stokes component varies as a function of wavelength across the entire spectrum is encoded into the signal of a single particular frequency channel. The more data points we have inside a given frequency channel, the finer spectral features we will be able to resolve in showing how that stokes component changes as a function of wavelength. By increasing the thickness of the retarders, the separation between the frequency channels is increased, providing more data points 
in a given channel. For thinner the retarders, there are fewer data points in each channel, resulting in the loss of resolution in the stokes component spectra.

This idea of stretching the frequency channels must also take into account the noise in the recorded images. Again our goal is to have an accurate measurement of the spectral

modulations. When the retarder thicknesses are increased, the channels are stretched out further, providing more data points across the spectra, but these modulations will now be at higher frequencies. Since only a few pixels are used to sample these higher frequencies, the measured frequency information will be more susceptible to noise. With a low signal level and high noise in these pixels, the modulations will not be accurately recorded and will result in errors in the reconstructed Stokes spectra. This issue can be overcome. You can still select the retarders to maximize the resolution of the reconstructed stokes spectrum but the signal from the object and the sensitivity of the camera must be large enough to overcome the noise.

So when selecting retarder thicknesses, it is useful for the designer to first find the maximum retarder thickness, where the produced fringes are no longer accurately measured, and then scale back the thicknesses to thinner values to try and meet the system performance requirements.

\subsection{Effect of dispersion of birefringence in calculating retarder thicknesses}

In the previous example of calculating the retarder thicknesses for an LWIR CTICS, it was stated that the material had a constant value of birefringence as a function of wavelength. This will not be true for many retarder materials. There will likely be at least some change in the birefringence as a function of wavelength. A similar process to the one outlined above can be used to determine the appropriate set of retarder thicknesses for retarder materials that have dispersion of birefringence.

The goal of the retarder thickness selections remains the same, to select retarder thicknesses that produce modulations across the spectra that can be accurately recorded by the CTICS system. If possible, it is useful to find the Sellmeier coefficients that describe the index of refraction for both the ordinary and extraordinary axes of the material over the specified wavelength region. By using the Sellmeier coefficients you can calculate the birefringence and retardance as a function of wavelength. Just as before, by associating a particular component of the outer frequency channel, like the center or the very edge of the channel, to the modulation limit of the system, a set of maximum values for the retarder thicknesses can be obtained. The primary difference is that the period of modulations in wavelength space must now be checked across the entire spectrum when determining the modulation limit as opposed to just at the lowest wavelength due to the varying birefringence.

The determined maximum thickness values can then be scaled to lower values to meet the sampling and signal requirements of the system. Again note that this modulation limit will be different for different objects, since the spectral resolution of the system is object dependent ${ }^{26}$, and the selection of retarders must be tailored to the types of objects viewed with the system.

\subsection{LWIR CTICS component selection details}


In the LWIR CTICS, the actual material used for the optical retarders is cadmium sulfide (CdS). The following Sellmeier coefficients describe the index of refraction for the ordinary and extraordinary axes of the material for wavelengths between 2.5 to 15 microns. ${ }^{27}$ A plot of the index of refraction for the ordinary and extraordinary axes of CdS is shown in figure 9.6.

\begin{tabular}{|c|c|c|}
\hline \multirow{2}{*}{$\begin{array}{c}\text { Sellmeier } \\
\text { Coefficients }\end{array}$} & \multicolumn{2}{|c|}{ Cds } \\
\hline & no & $\mathrm{Ne}$ \\
\hline A & 3.7255 & 3.6522 \\
\hline$B$ & 1.4491 & 1.5975 \\
\hline C & 0.16339 & 0.14526 \\
\hline$D$ & 1.2612 & 1.4869 \\
\hline$E$ & 733.21 & 794.56 \\
\hline
\end{tabular}

Table 6: Sellmeier coefficients describing the index of refraction for CdS

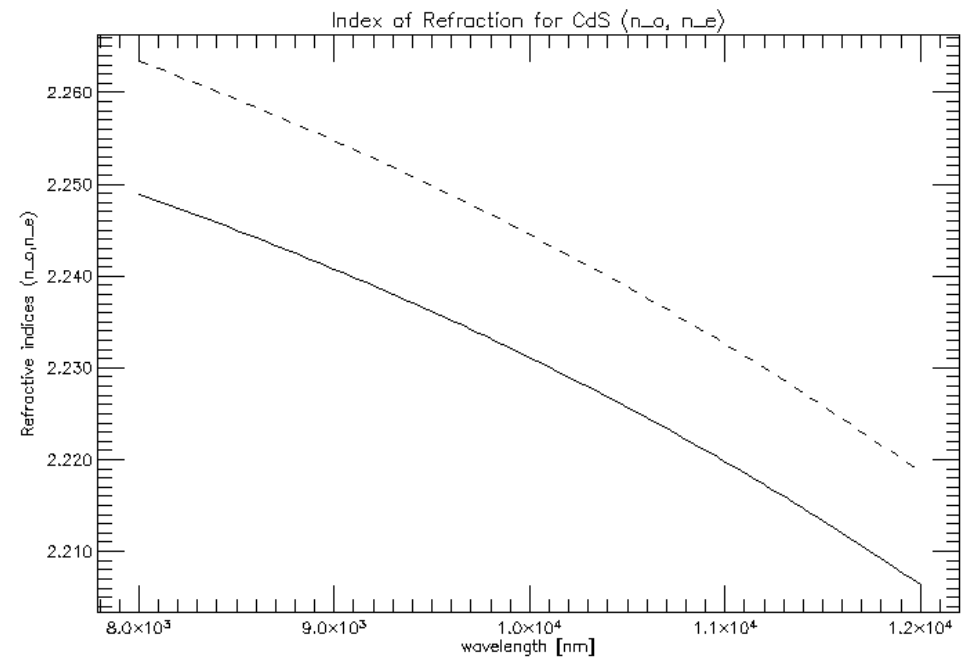

Figure 9.6: Index of Refraction for both the ordinary (solid line) and extraordinary (dashed-line) optical axes of Cadmium Sulfide, CdS.

As seen in figure 9.6, cadmium sulfide does have some dispersion of birefringence. Between the wavelengths of 8 to 12 microns, the value of the birefringence varies from .0145 to .0122 .

To determine the set of thickness values that corresponds to the modulation limit for this material, we begin by using the same procedure as the previous example for a material with no dispersion of birefringence, but select a single fixed value of birefringence that falls within the 
range of actual values. For this case we will select a birefringence value of 0.0140 and the corresponding thickness of the first retarder is calculated as follows.

$$
\begin{aligned}
& d_{1 \text { first guess }}=\frac{\lambda_{\min }^{2}+\lambda_{\min } \Delta \lambda}{3 \Delta \lambda \Delta n} \\
& d_{1 \text { first guess }}=\frac{(8000 \mathrm{~nm})^{2}+(8000 \mathrm{~nm})(120 \mathrm{~nm})}{(3)(120 \mathrm{~nm})(0.0140)}=12.89 \mathrm{~mm}
\end{aligned}
$$

By using the actual birefringence values as a function of wavelength given by the Sellmeier coefficients, we can check to see if the produced modulation periods for this retarder thickness are greater than the spectral resolution of the spectrometer system for all the modulations across the entire spectra. In this example, using $\mathrm{CdS}$ retarders with a 1:2 thickness ratio and a thickness of $12.89 \mathrm{~mm}$ for the first retarder, there are 12 fringes produced that have a period of less than $120 \mathrm{~nm}$ (two times the spectral sampling rate of $60 \mathrm{~nm}$ for the LWIR system) within the selected wavelength region. The thickness of the retarders must be decreased, reducing the number of modulations across the spectrum, so all of the modulations can be accurately recorded by the system.

By decreasing the thickness of the first retarder to a value of $10.00 \mathrm{~mm}$, the shortest period of modulation produced is found to be equal to the spectral resolution of the system in wavelength space. Since all of the other modulations across the spectra will have a period greater than the spectral resolution of the system, the produced spectra should be right at the limit of accurate detection for the system.

These results were confirmed by using a custom computer simulation program written for channeled spectropolarimeter systems. The simulation software, shown in figure 9.7, allows the modeling of different input spectra, noise effects, different retarder thicknesses and materials, and the use of different windowing functions during the reconstruction. The selection of thinner retarders was guided by the results of this software after looking at the effects of noise on the reconstructions and seeing how the resolution of the reconstructed stokes spectra is decreased for thinner retarders. 


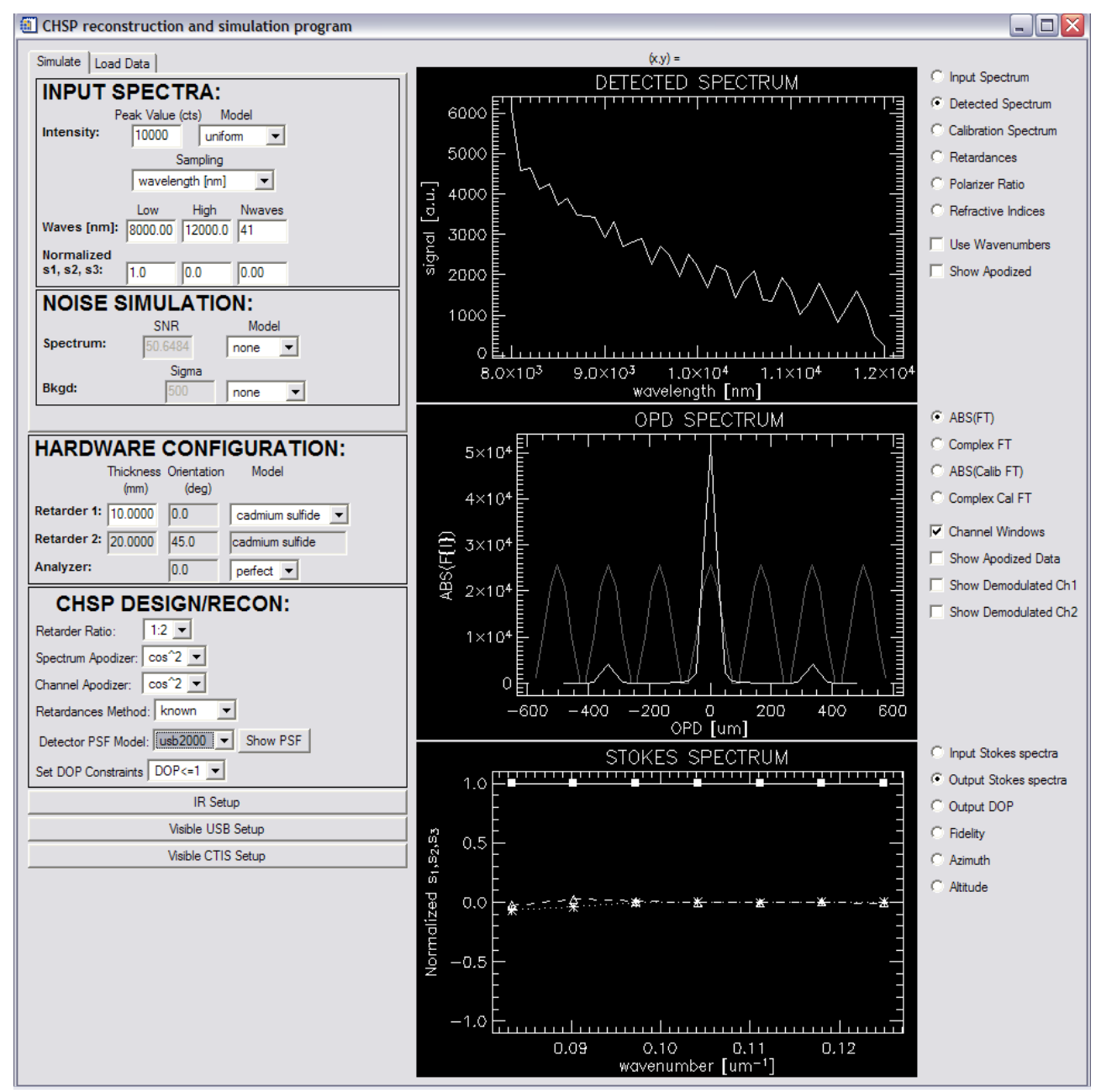

Figure 9.7: Simulation program to model the channeled spectropolarimeter system

Since the LWIR CTICS system was intended to be a proof of concept, three different cadmium sulfide retarders were ordered for the system: a $5 \mathrm{~mm}$ thick retarder, a $10 \mathrm{~mm}$ thick retarder, and a $20 \mathrm{~mm}$ thick retarder. The combination of the $10 \mathrm{~mm}$ and $20 \mathrm{~mm}$ retarders is intended to be able to demonstrate the maximum performance of the system, provided a bright enough point object is viewed to overcome the noise in the system, while the $5 \mathrm{~mm}$ and $10 \mathrm{~mm}$ retarders will allow the system to be used for a wider variety of objects but with a loss of spectral resolution in the reconstructed stokes spectra. Unfortunately, the manufacturer was unable to produce the $20 \mathrm{~mm}$ thick retarder. So only the $5 \mathrm{~mm}$ and $10 \mathrm{~mm}$ CdS retarders were used in the LWIR CTICS system.

\subsection{Measurements and Results from the LWIR CTICS}

The results from the LWIR CTICS demonstrate that snapshot imaging spectropolarimetry in the 8 to 12 micron region works. The ability of the system to simultaneously record both the spectral and polarimetric signatures of all the spatial locations/targets in a scene with just a single integration period of a camera is shown in the following data sets for figures 9.8, 9.9, and 9.10. A photo of the constructed system is shown in figure 9.11. 

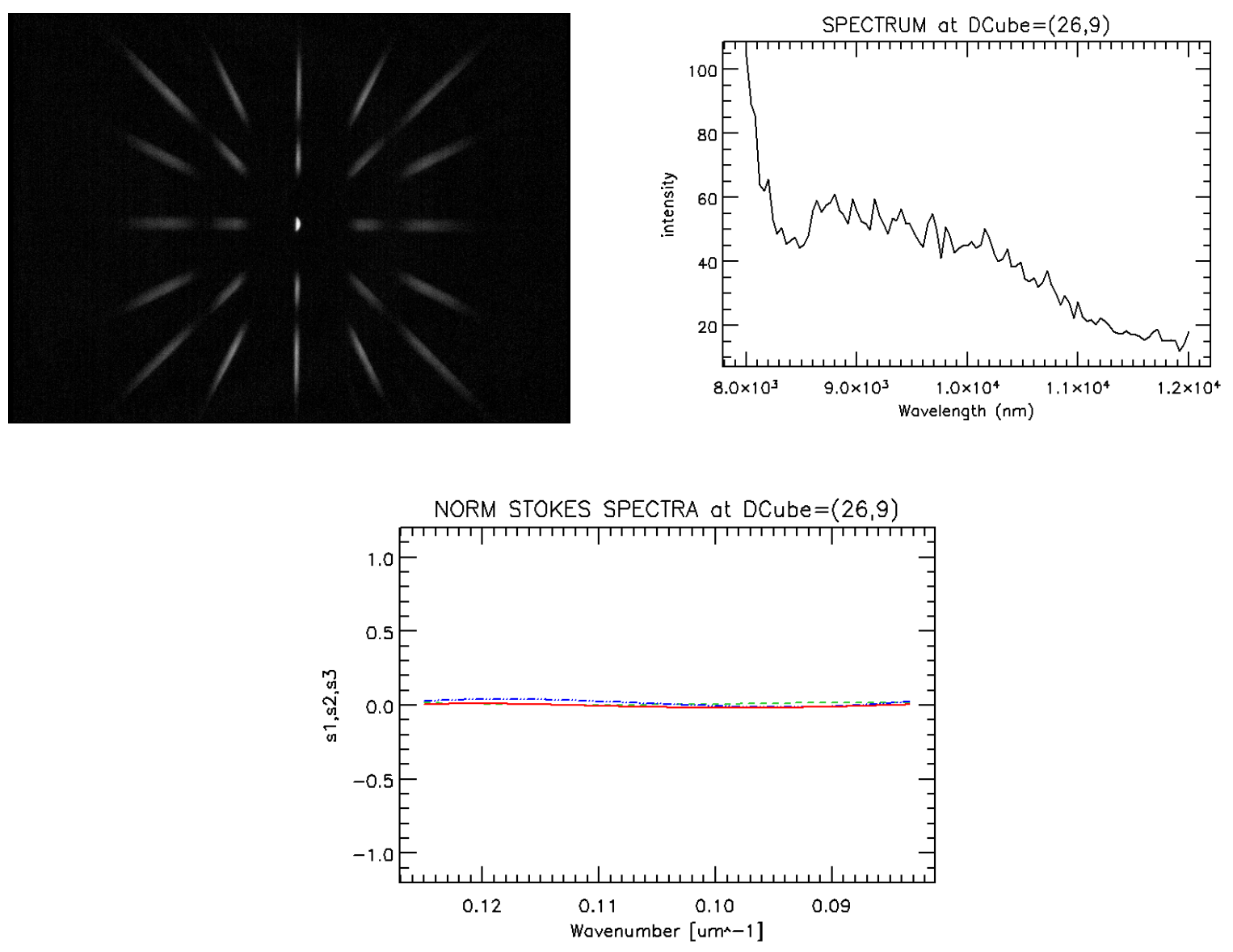

Figure 9.8: Unpolarized Blackbody Object; (top left) Raw CTICS image, (top right) Reconstructed spectrum, (bottom) Normalized reconstructed stokes spectra. Note that S1, S2, and $\mathrm{S} 3$ are all zero across the spectra as expected for an unpolarized object 

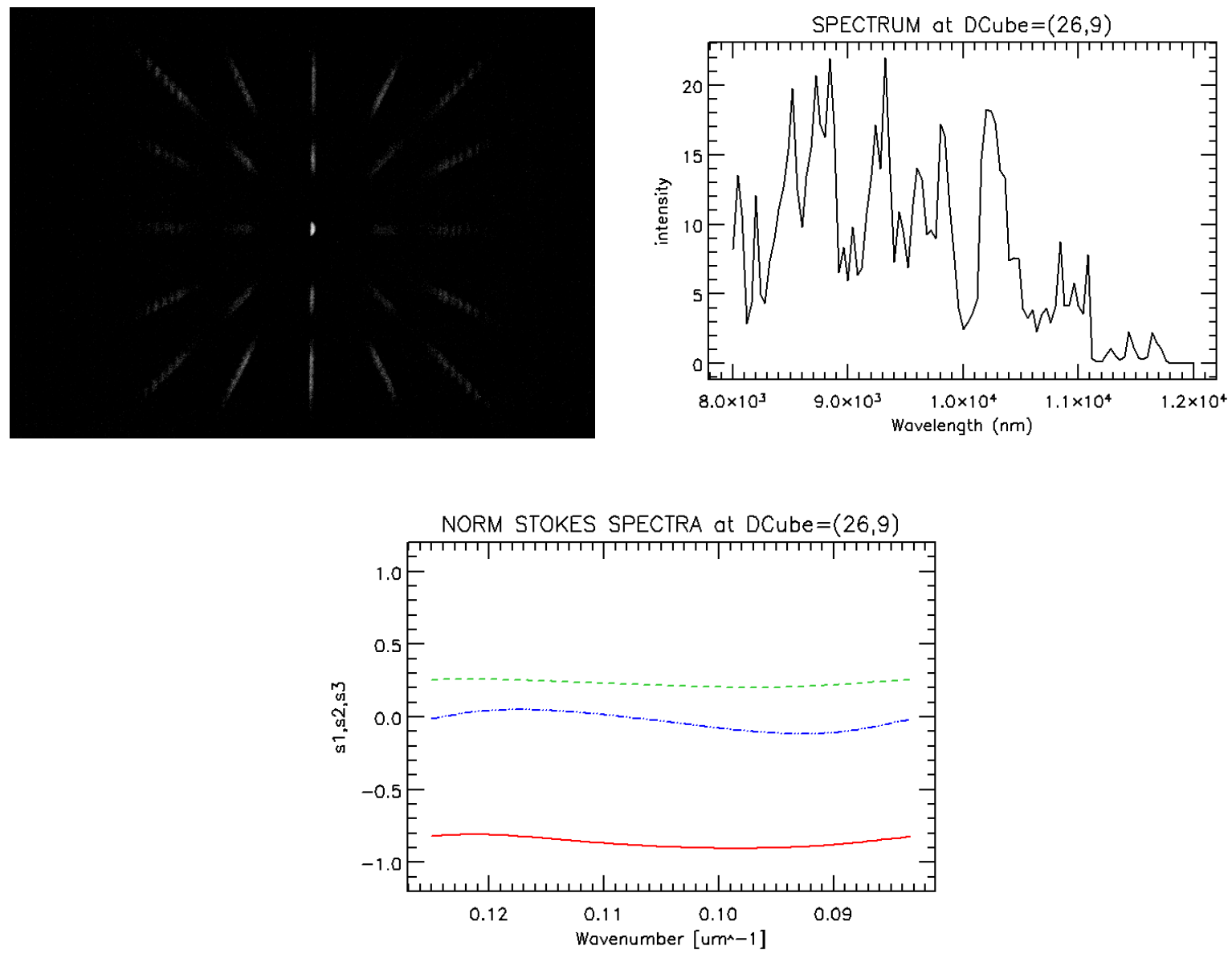

Figure 9.9: Vertically Polarized Blackbody Object; (top left) Raw CTICS image, (top right) Reconstructed spectrum, (bottom) Normalized reconstructed stokes spectra. Note that S1 is close to -1 across the entire spectra, as expected, while S2 and S3 are all close zero across the spectra 

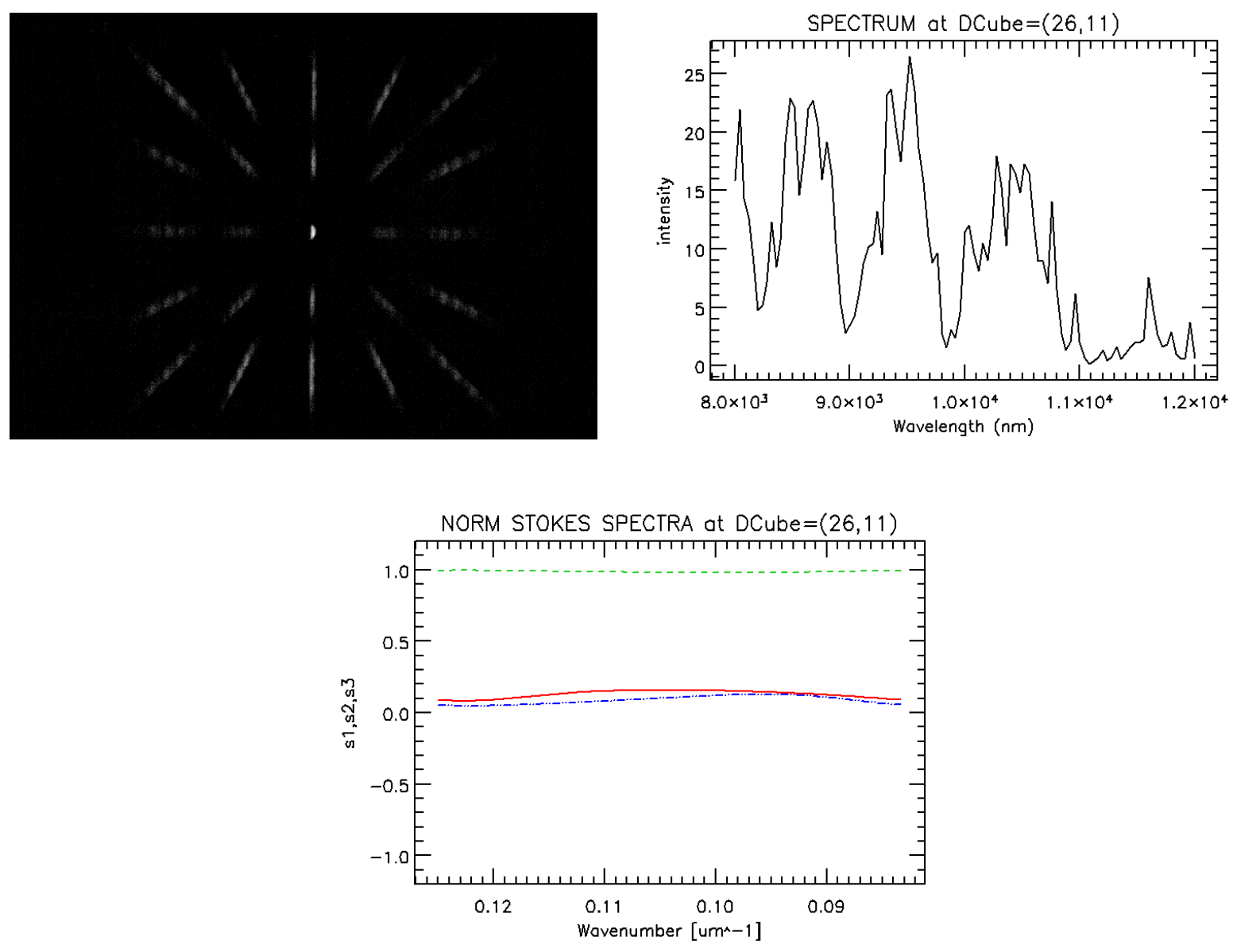

Figure 9.10: Blackbody Polarized at 45 Degrees; (top left) Raw CTICS image, (top right) Reconstructed spectrum, (bottom) Normalized reconstructed stokes spectra. Note that S2 is 1 across the entire spectra, as expected, while S2 and S3 are both close zero across the spectra

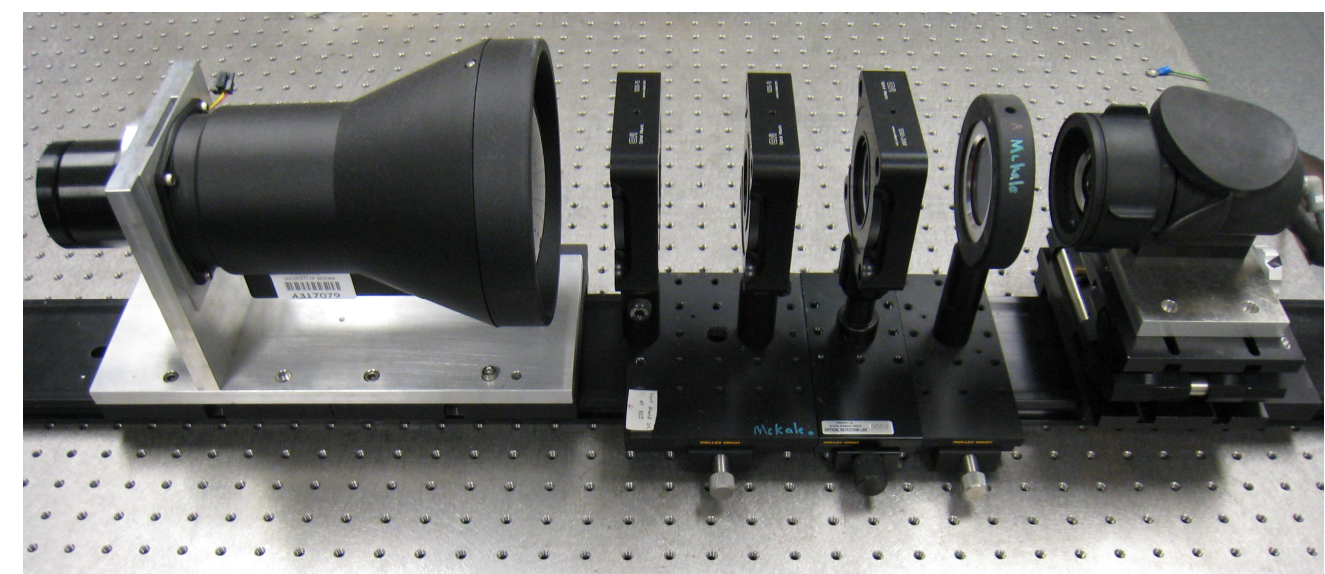

Figure 9.11: Photo of the assembled LWIR CTICS system. Components include off the shelf LWIR lenses, crystal retarders, a wire grid polarizer, GaAs CGH, and a 640x480 resolution uncooled silicon microbolometer LWIR camera. 


\section{CHAPTER 10: CONCLUSION}

\subsection{Closing Remarks}

The primary goal of this dissertation research has been to develop and demonstrate a functioning proof-of-concept snapshot imaging spectropolarimeter system for the LWIR 8-12 micron wavelength region of the electromagnetic spectrum. The constructed LWIR CTICS system has met this goal.

The optical system combines the technology of computed tomographic imaging spectrometry (CTIS) with channeled spectropolarimetry and has demonstrated its ability to simultaneously measure both the spectral and polarimetric signatures of all the spatial locations/targets in a scene with just a single integration period of the camera. The LWIR CTICS system contains no moving parts and collects complete spatial, spectral, polarimetric data in a single snapshot. This snapshot capability makes it uniquely suited for observing and characterizing both the spectral and polarimetric features of quick events or fast moving objects without the associated scanning artifacts found in conventional imaging spectrometers and polarimeters.

The constructed system is the first instrument of this type specifically designed to operate in the long wavelength infrared region, as well as being the first demonstration of such a system using an uncooled infrared focal plane array.

In addition to this proof-of-concept demonstration, this dissertation research has also focused on a variety of methods that can significantly improve CTIS system performance. A detailed stepby-step process has been presented for the first order optical design of CTIS systems including approaches to design "outer ring" diffraction patterns as well as clearly specifying the specific rules that need to be followed when tweaking a design based on component fabrication abilities and availability. Details on the selection and design of the CGH disperser were covered describing how the CGH properties and diffraction patterns could be tailored for particular hyperspectral detection applications. A new simplified approach to the calibration of a CTIS system was presented outlining the generation of synthetic calibration images based on a limited number of physical system measurements. This dissertation has also outlined some of the newly uncovered unique mathematical properties of CTIS H matrices, namely the possibility of forming the $\mathrm{H}$ matrix as a sparse block Toeplitz matrix as well as the ability to determine the left inverse of the H matrix for many CTIS systems. For CTICS systems, a detailed selection process has been presented for finding the optimal retarder thicknesses. However, despite these improvements there has been one problem that has been preventing the use of CTIS and CTICS in many applications for over a decade now: the extremely slow reconstruction process. So perhaps the most significant performance improvements produced by this dissertation research are the outlined methods to dramatically increase the speed of CTIS reconstructions. Through this dissertation research new methods have been developed and implemented that allowed for the first demonstration of a CTIS system capable of computing reconstructions in "real time." This development could greatly expand the use of CTIS in the future, and as more computing resources become available, the dissertation has outlined multiple ways to even further improve the speed of CTIS reconstructions when using either sequential or parallel reconstruction processing. 


\section{APPENDIX A: DETAILS ON TRANSFORM OF CGH TRANSMITTANCE}

\section{The Fourier Transform of the CGH Transmittance Function}

The CGH transmission is given by the convolution of three separate functions.

$$
\begin{aligned}
& t_{\text {Entire.CGH }}(\xi, \eta)=\sum_{m, n=0}^{q-1} e^{j \Phi m n} \delta\left(\xi-m \xi_{c}\right) \delta\left(\eta-n \eta_{c}\right) * * \\
& \operatorname{rect}\left(\frac{\xi}{\xi_{c}}\right) \operatorname{rect}\left(\frac{\eta}{\eta_{c}}\right) * * \\
& \sum_{k, l=-\infty}^{\infty} \delta\left(\xi-k\left(q \xi_{c}\right)\right) \delta\left(\eta-l\left(q \eta_{c}\right)\right)
\end{aligned}
$$

This section provides details on how the Fourier transform of this CGH transmission can be

calculated as:

$$
\begin{aligned}
\left.\mathfrak{J}\left\{t_{\text {Entire.CGH}}(\xi, \eta)\right\}\right|_{x^{\prime}=\frac{x}{\lambda \mathrm{f}}, \mathrm{y}^{\prime}=\frac{y}{\lambda \mathrm{f}}=} & \sum_{m, n=0}^{q-1} e^{j \Phi m n} e^{-j 2 \pi m \xi_{c} \frac{x}{\lambda f}} e^{-j 2 \pi n \eta_{c} \frac{y}{\lambda f}} \times \\
& \left|\xi_{c}\right|\left|\eta_{c}\right| \operatorname{sinc}\left(\frac{\xi_{c} x}{\lambda f}\right) \operatorname{sinc}\left(\frac{\eta_{c} y}{\lambda f}\right) \times \\
& \sum_{k, l=-\infty}^{\infty} \delta\left(x-\frac{k \lambda f}{q \xi_{c}}\right) \delta\left(y-\frac{l \lambda f}{q \eta_{c}}\right)
\end{aligned}
$$

The convolution theorem states that the Fourier transform of a convolution of two functions is the product of the Fourier transforms of the functions. So when taking the Fourier transform of the CGH transmission equation and also using the associative property, the resulting expression will be the product of three Fourier transforms.

In the first term, the Fourier transform of the finite number of delta functions will just be phase terms.

$$
\begin{aligned}
& \left.\mathfrak{I}\left\{\sum_{m, n=0}^{q-1} e^{j \Phi m n} \delta\left(\xi-m \xi_{c}\right) \delta\left(\eta-n \eta_{c}\right)\right\}\right|_{x^{\prime}=\frac{x}{\lambda \mathrm{f}}, \mathrm{y}^{\prime}=\frac{y}{\lambda \mathrm{f}}}=\sum_{m, n=0}^{q-1} e^{j \Phi m n} e^{-j 2 \pi m \xi_{c} \frac{x}{\lambda f}} e^{-j 2 \pi m \eta_{c} \frac{y}{\lambda f}} \\
& \mathfrak{I}\{\delta(\xi-b)\}=e^{-j 2 \pi b x}
\end{aligned}
$$


The Fourier transform of the rect functions in the second terms will be sinc functions.

$$
\begin{aligned}
& \left.\mathfrak{I}\left\{\operatorname{rect}\left(\frac{\xi}{\xi_{c}}\right) \operatorname{rect}\left(\frac{\eta}{\eta_{c}}\right)\right\}\right|_{x^{\prime}=\frac{x}{\lambda \mathrm{f}}, \mathrm{y}^{\prime}=\frac{y}{\lambda \mathrm{f}}}=\left|\xi_{c}\right|\left|\eta_{c}\right| \operatorname{sinc}\left(\frac{\xi_{c} x}{\lambda f}\right) \operatorname{sinc}\left(\frac{\eta_{c} y}{\lambda f}\right) \\
& \mathfrak{I}\{\operatorname{rect}(\xi)\}=\operatorname{sinc}(x) \\
& F\left(\frac{\xi}{d}\right) \Rightarrow|d| f(d x) \\
& F_{1}(\xi) F_{2}(\eta) \Rightarrow f_{1}(x) f_{2}(y)
\end{aligned}
$$

The third term is an infinite number of delta functions equally spaced apart, also called a comb function. The Fourier transform of a comb function will be another comb function with different spacing. The following steps will specifically evaluate the Fourier transform of this third term and show how the result is calculated.

The comb function can be defined as follows.

$$
\operatorname{comb}\left(\frac{\mathrm{x}}{b}\right) \Rightarrow \mid b\left[\left[\sum_{n=-\infty}^{\infty} \delta(x-n b)\right]\right.
$$

Which can be rewritten as

$$
\sum_{n=-\infty}^{\infty} \delta(x-n b)=\frac{1}{|b|} \operatorname{comb}\left(\frac{\mathrm{x}}{b}\right)
$$

So the third term of the transmission function can be expressed as

$$
\sum_{k, l=-\infty}^{\infty} \delta\left(\xi-k\left(q \xi_{c}\right)\right) \delta\left(\eta-l\left(q \eta_{c}\right)\right)=\frac{1}{\left|q \xi_{c}\right|} \operatorname{comb}\left(\frac{\xi}{q \xi_{c}}\right) \frac{1}{\left|q \eta_{c}\right|} \operatorname{comb}\left(\frac{\eta}{q \eta_{c}}\right)
$$

By using the following Fourier transform pair and the separability property:

$$
F\left(\frac{\xi}{d}\right) \Rightarrow|d| f(d x) \quad \text { and } \quad F_{1}(\xi) F_{2}(\eta) \Rightarrow f_{1}(x) f_{2}(y)
$$

The infinite sum of delta functions in the third term of equation (A.1) can be separable into two components. Equations (A.6) and (A.7) show how each separable component can be reexpressed as a single comb function.

$$
\mathfrak{I}\left\{\frac{1}{\left|q \xi_{c}\right|} \operatorname{comb}\left(\frac{\xi}{q \xi_{c}}\right)\right\}=\left[\frac{1}{\left|q \xi_{c}\right|}\right]\left|q \xi_{c}\right| \operatorname{comb}\left(q \xi_{c} x\right)=\operatorname{comb}\left(q \xi_{c} x\right)
$$


So the Fourier transform of each component of the third term of equation (A.1) evaluated at $\xi=\mathrm{x}$ / $(\lambda \mathrm{f})$ can be determined as follows:

$$
\left.\mathfrak{I}\left\{\sum_{k=-\infty}^{\infty} \delta\left(\xi-k\left(q \xi_{c}\right)\right)\right\}\right|_{\xi=\frac{x}{\lambda f}}=\operatorname{comb}\left(q \xi_{c} \frac{x}{\lambda f}\right)=\sum_{k=-\infty}^{\infty} \delta\left(\frac{x q \xi_{c}}{\lambda f}-k\right)
$$

The result is an infinite sum of delta function for each component of the third term of equation (A.1). The location of these delta functions will occur when the term inside the delta function is equal to zero. This central term inside the delta function can be rewritten to isolate the $\mathrm{x}$ component.

$$
\frac{x q \xi_{c}}{\lambda f}-k=0 \Rightarrow x-\frac{k \lambda f}{q \xi_{c}}=0
$$

So the net result is that the Fourier transform of the third term of equation (A.1) can be expressed as follows:

$$
\left.\mathfrak{I}\left\{\sum_{k, l=-\infty}^{\infty} \delta\left(\xi-k\left(q \xi_{c}\right)\right) \delta\left(\eta-l\left(q \eta_{c}\right)\right)\right\}\right|_{\xi=\frac{x}{\lambda \mathrm{f}}, \eta=\frac{y}{\lambda \mathrm{f}}}=\sum_{k, l=-\infty}^{\infty} \delta\left(x-\frac{k \lambda f}{q \xi_{c}}\right) \delta\left(y-\frac{l \lambda f}{q \eta_{c}}\right)
$$

Putting this result for the third term together with the Fourier transform of the other terms of equation (A.1), the Fourier transform of the entire CGH transmittance function evaluated at $\mathrm{x}^{\prime}=$ $\mathrm{x} / \lambda \mathrm{f}$ and $\mathrm{y}^{\prime}=\mathrm{y} / \lambda \mathrm{f}$ can be expressed as:

$$
\begin{aligned}
\left.\mathfrak{I}\left\{t_{\text {Entire.CGH}}(\xi, \eta)\right\}\right|_{x^{\prime}=\frac{x}{\lambda \mathrm{f}}, y^{\prime}=\frac{y}{\lambda \mathrm{f}}=} & \sum_{m, n=0}^{q-1} e^{j \Phi m n} e^{-j 2 \pi m \xi_{c} \frac{x}{\lambda f}} e^{-j 2 \pi n \eta_{c} \frac{y}{\lambda f}} \times \\
& \left|\xi_{c}\right|\left|\eta_{c}\right| \operatorname{sinc}\left(\frac{\xi_{c} x}{\lambda f}\right) \operatorname{sinc}\left(\frac{\eta_{c} y}{\lambda f}\right) \times \\
& \sum_{k, l=-\infty}^{\infty} \delta\left(x-\frac{k \lambda f}{q \xi_{c}}\right) \delta\left(y-\frac{l \lambda f}{q \eta_{c}}\right)
\end{aligned}
$$




\section{APPENDIX B: THE SHOELACE ALGORITHM}

\section{The Shoelace Algorithm}

There is a simple algorithmic process that can be used to determine the enclosed area of a polygon. This process works for any simple polygon, regardless of the number of sides, and is sometimes referred to as the shoelace method. By listing the vertices of the polygon counterclockwise around the perimeter as $\left(\mathrm{x}_{0}, \mathrm{y}_{0}\right),\left(\mathrm{x}_{1}, \mathrm{y}_{1}\right), \ldots,\left(\mathrm{x}_{\mathrm{n}-1}, \mathrm{y}_{\mathrm{n}-1}\right)$, the area of the polygon is:

$$
A=\frac{1}{2}\left\{\left|\begin{array}{ll}
x_{0} & x_{1} \\
y_{0} & y_{1}
\end{array}\right|+\left|\begin{array}{ll}
x_{1} & x_{2} \\
y_{1} & y_{2}
\end{array}\right|+\cdots+\left|\begin{array}{ll}
x_{n-2} & x_{n-1} \\
y_{n-2} & y_{n-1}
\end{array}\right|+\left|\begin{array}{ll}
x_{n-1} & x_{0} \\
y_{n-1} & y_{0}
\end{array}\right|\right\}
$$

Why this algorithm is sometimes called the shoelace method can be seen in the following example, taken from the $2^{\text {nd }}$ volume of the IMSA Math Journal published in fall of $1993 .{ }^{28}$

\section{Shoelace Algorithm Example}

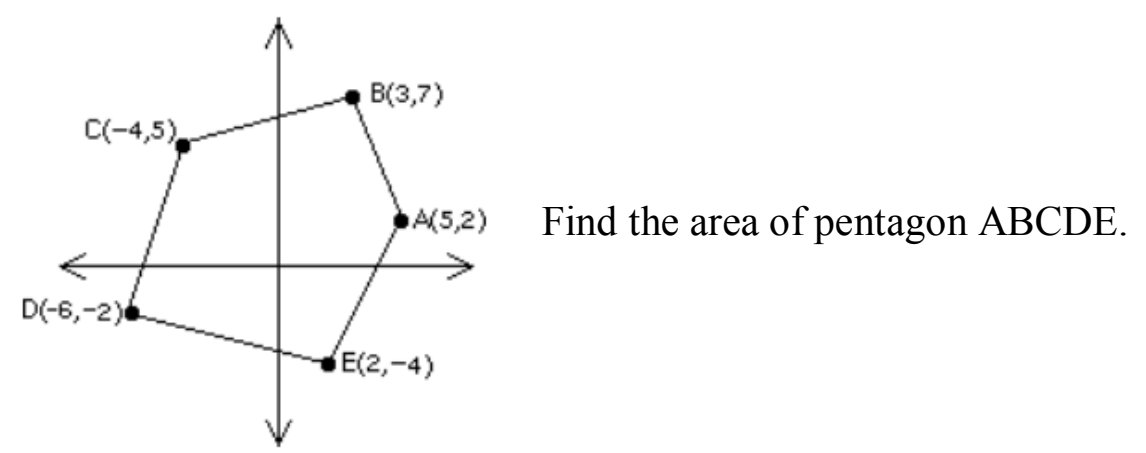

The area, K, can be found by following the procedure:

1) Select a vertex and travel around the pentagon ending with the starting point. Write a matrix of the coordinates of the path including the starting and ending coordinates.

$$
\left[\begin{array}{r:r}
2 & -4 \\
5 & 2 \\
3 & 7 \\
-4 & 5 \\
-6 & -2 \\
2 & -4
\end{array}\right]
$$


2) Determine the sum of all “ $\backslash$ ” products: $(2)(2)+(5)(7)+(3)(5)+(-4)(-2)+(-6)(-4)$

$=4+35+15+8+24$

$=86$

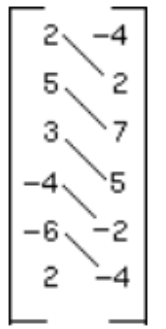

3) Determine the sum of all " / " products: $(5)(-4)+(3)(2)+(-4)(75)+(-6)(5)+(2)(-2)$

$$
=-20+6-28-30-4
$$

$=-76$

$\left[\begin{array}{lr}2 & -4 \\ 5 & 5^{2} \\ 3 & 7 \\ -4 & r^{7} \\ -6 & -2 \\ 2 & -4 \\ - & \end{array}\right]$

4) Determine the absolute value of the difference of the "I" products and the "/" products: $\operatorname{abs}((86)-(-76))=\operatorname{abs}(162)$

$$
=162
$$

5) Take $1 / 2$ of the result from (4).

$$
1 / 2(162)=81[\text { area of pentagon] }
$$

The formula for the area is displayed:

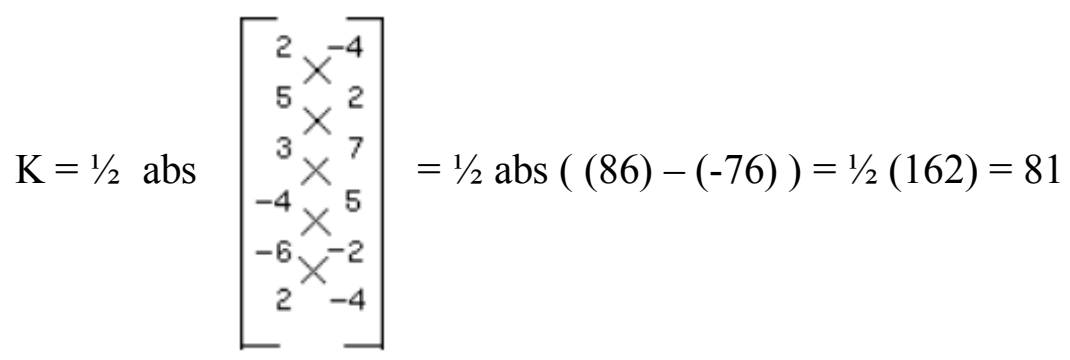

Note: Do you see the "shoelaces"? 


\section{REFERENCES}

[1] J. S. Tyo, D. L. Goldstein, D. B. Chenault, and J. A. Shaw, "Review of passive imaging polarimetry for remote sensing applications," Appl. Opt. 45, 5453-5469, 2006.

[2] C. Vandervlugt, N. Hagen, R. Sampson, E. Dereniak, G. Gerhart, "Visible imaging spectropolarimeter," Proceedings of SPIE, Volume 6661, Imaging Spectrometry XII, S. S. Shen, P. E. Lewis, Editors, San Diego, CA, 6661042007.

[3] A.M. Locke, D. Salyer, D. S. Sabatke; E. Dereniak, "Design of a SWIR computed tomographic imaging channeled spectropolarimeter." Proceedings of SPIE. Vol. 5158. 2003.

[4] C. E. Volin, T. M. Gleeson, M. R. Descour, E. Dereniak, "Portable computed-tomography imaging spectrometer." SPIE's 1996 International Symposium on Optical Science, Engineering, and Instrumentation. International Society for Optics and Photonics, 1996.

[5] K. Oka, "Singleshot spectroscopic polarimetry using channeled spectrum," in Advanced Materials and Devices for Sensing and Imaging, J. Yao and Y. Ishii, eds., Proc. SPIE 4919, pp. 167-175, 2002.

[6] K. Oka, T. Kato, "Spectroscopic polarimetry with a channeled spectrum," Optics Letters 24, pp. 1475-1477, 1999.

[7] A. M. Locke, Design and analysis of a snapshot imaging spectropolarimeter. $\mathrm{PhD}$ thesis, University of Arizona, Tucson, Arizona, 2003.

[8] N. Hagen, Snapshot Imaging Spectropolarimetry. PhD thesis, Univ. of Arizona, 2007.

[9] M. R. Descour, Non-scanning imaging spectrometry. PhD thesis, University of Arizona, 1994

[10] C. E. Volin, M. R. Descour, E. Dereniak. "Design of broadband-optimized computergenerated hologram dispersers for the computed-tomography imaging spectrometer." Proc. SPIE. Vol. 4480. 2002.

[11] J. W. Goodman, Introduction to Fourier Optics, Roberts \& Company Publishers, Colorado, Third Edition, 2005, p. 105.

[12] J. W. Goodman, Introduction to Fourier Optics, Roberts \& Company Publishers, Colorado, Third Edition, 2005, p. 107.

[13] R.W. Gerchberg and W.O Saxton, "A practical algorithm for the determination of phase from image and diffraction plane pictures," Opti 35, pp. 237-246, 1972.

[14] C. E. Volin, Portable snapshot infrared imaging spectrometer. $\mathrm{PhD}$ thesis, University of Arizona, Tucson, Arizona, 2000. 
[15] N. Hagen, E. L. Dereniak, and D. T. Sass, "Maximizing the resolution of a CTIS instrument," in Imaging Spectrometry XI, S. S. Shen and P. E. Lewis, eds., Proc. SPIE 6302, 36020L, 2006.

[16] N. Hagen, Snapshot Imaging Spectropolarimetry. PhD thesis, Univ. of Arizona, 2007.

[17] N. Hagen, E. Dereniak, "New grating designs for a CTIS imaging spectrometer," Proceedings of SPIE, Volume 6565, Algorithms and Technologies for Multispectral, Hyperspectral, and Ultraspectral Imagery XIII, S. S. Shen and P. E. Lewis, editors, Orlando, FL, 65650N, 2007.

[18] Michael W. Kudenov ; Julia Craven-Jones ; Riley Aumiller ; Corrie Vandervlugt and Eustace L. Dereniak "Faceted grating prism for a computed tomographic imaging spectrometer", Opt. Eng. 51(4), 044002, 2012.

[19] N. Hagen, E. Dereniak, "Analysis of computed tomographic imaging spectrometers. I. Spatial and spectral resolution," Applied Optics, Vol. 47, No. 28, 2008.

[20] J. Scholl, The Design and Analysis of Computer Tomographic Imaging Spectrometers (CTIS) Using Fourier and Wavelet Crosstalk Matrices. PhD thesis, University of Arizona, 2010.

[21] N. Hagen, Snapshot Imaging Spectropolarimetry. PhD thesis, Univ. of Arizona, 2007.

[22] N. Hagen, Snapshot Imaging Spectropolarimetry. PhD thesis, Univ. of Arizona, 2007.

[23] Nvidia corporation, Tesla M2090 Product brief, [online] 2011, http://www.nvidia.com/docs/IO/105880/DS_Tesla-M2090_LR.pdf

[24] Nvidia corporation, Tesla M-Class GPU Computing Modules, Accelerating Science, [online] 2011, http://www.nvidia.com/docs/IO/105880/DS-Tesla-M-Class-Aug11.pdf

[25] N. Hagen, E. Dereniak, "Analysis of computed tomographic imaging spectrometers. I. Spatial and spectral resolution," Applied Optics, Vol. 47, No. 28, 2008.

[26] N. Hagen, E. Dereniak, "Analysis of computed tomographic imaging spectrometers. I. Spatial and spectral resolution," Applied Optics, Vol. 47, No. 28, 2008.

[27] D. B. Chenault and R. A. Chipman, "Infrared Birefringence Spectra for Cadmium-Sulfide and Cadmium Selenide," Appl. Opt. 32, 4223-4227, 1993.

[28] C. L. Hamberg and R. Vavrinek, "Shoelace Algorithm," Illinois Mathematics and Science Academy IMSA Math Journal, Fall 1993. 